\title{
The significance of coherent flow structures for the turbulent mixing in wall-bounded flows
}

\author{
Dissertation \\ zur Erlangung des Doktorgrades der \\ Mathematisch-Naturwissenschaftlichen Fakultäten der \\ Georg-August-Universität zu Göttingen
}

\author{
Vorgelegt von \\ Christian J. Kähler \\ aus Buchholz i. d. Nordheide
}

Göttingen 2004 
D7

Referent: Professor Dr. H. Eckelmann Korreferent: Professor Dr. D. Ronneberger Tag der mündlichen Prüfung: 1. July 2004 


\section{Contents}

1 Introduction $\quad 5$

2 Particle Image Velocimetry 13

2.1 Principles . . . . . . . . . . . . . . . . . . 13

2.2 Generation of appropriate tracer-particles . . . . . . . . . . . . 16

2.2.1 Description of the experiment . . . . . . . . . . . 16

2.2 .2 Particle size analysis . . . . . . . . . . . . . . . . . . 17

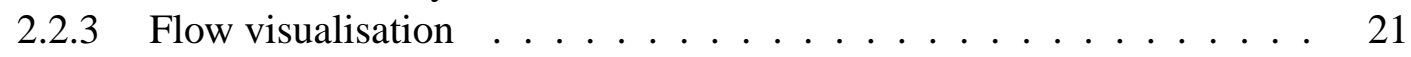

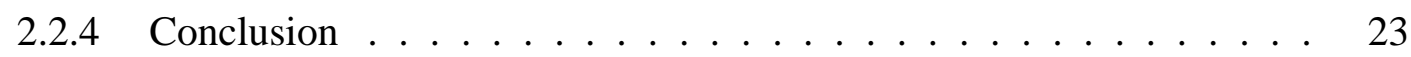

2.3 Registration of the particle images . . . . . . . . . . . . . 23

2.3.1 Principles of CCD sensors ........................ 24

2.3.2 Quantum efficiency and signal-to-noise ratio .......... 25

2.3 .3 CCD architecture . . . . . . . . . . . . . . 26

2.4 Particle image analysis . . . . . . . . . . . . . . . . . . 28

2.4.1 Particle image density, loss of pairs and velocity gradients . . . . 30

2.4.2 Signal-peak detection and displacement determination . . . . . . . 31

3 Stereo-scopic Particle Image Velocimetry 37

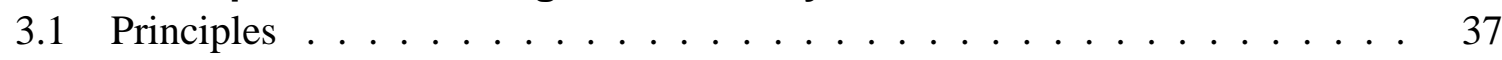

3.1 .1 Error analysis . . . . . . . . . . . . . . . . 39

3.1.2 Scheimpflug condition . . . . . . . . . . . . . . 41

3.2 Evaluation of stereo-scopic image pairs . . . . . . . . . . . . . 42

3.2.1 Determination of the mapping function . . . . . . . . . . . . . 42

3.2 .2 Image warping . . . . . . . . . . . . . . . . . . . . 43

3.2 .3 Vector field warping . . . . . . . . . . . . . . 45

3.2 .4 Interrogation window warping . . . . . . . . . . . . 45

3.3 Calibration validation . . . . . . . . . . . . . 46

4 Multiplane Stereo Particle Image Velocimetry 49

4.1 Principles . . . . . . . . . . . . . . . . . . . . . 49

4.2 Four-pulse-laser System $\ldots \ldots \ldots \ldots$. . . . . . . . . . . . 51

4.2.1 Performance of spatial light-sheet separation . . . . . . . . . . 53

4.2.2 Generation and controlling of the timing sequence ........ 54

4.3 Modes of Operation I - In-plane flows . . . . . . . . . . . . . . . . . 55

4.4 Modes of Operation II - Out-of-plane flows . . . . . . . . . . . . . . . 57

4.5 Simplified recording system . . . . . . . . . . . . . . . . . . . . . . . . . . . . 59

4.6 Polarisation effects . . . . . . . . . . . . . . . . 59 
4.7 Monochromatic aberrations . . . . . . . . . . . . . . . . . 61

4.8 Feasibility study . . . . . . . . . . . . . . . . . . 65

5 Investigation of the xy-plane 69

5.1 The statistical description of turbulence . . . . . . . . . . . . . 69

5.2 Experimental set-up . . . . . . . . . . . . . . . . 72

5.3 Statistical properties of the flow . . . . . . . . . . . 75

$5.3 .1 \quad$ Single point statistics . . . . . . . . . . . . . . 75

5.3.2 Spatial auto- and cross-correlation functions . . . . . . . . . . . 79

5.4 Properties of coherent velocity structures . . . . . . . . . . . . . . . . . 90

5.4 Shear-layer . . . . . . . . . . . . . . . . 90

5.4 Ejection . . . . . . . . . . . . . . . 93

5.4 .3 Sweep . . . . . . . . . . . . . . . . . 95

6 Investigation of the xz-plane $\quad 97$

6.1 Experimental set-up . . . . . . . . . . . . . . . . . . . . . . 98

6.2 Statistical properties of the buffer layer . . . . . . . . . . . . . . . 101

6.2 .1 Single point statistics . . . . . . . . . . . . . . . . . . . 101

6.2.2 Spatial auto-correlation functions . . . . . . . . . . . . . . 105

6.2.3 Spatial cross-correlation functions . . . . . . . . . . . . . . . 109

6.3 Spatio-temporal buffer layer statistics . . . . . . . . . . . . . . . . . . . . 114

6.4 Properties of coherent velocity structures . . . . . . . . . . . . . . . . . . . . . . . . . . . . 121

6.4.1 Low-speed streaks . . . . . . . . . . . . . . . . . . 121

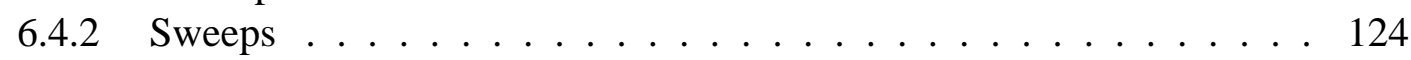

6.4 .3 Ejection . . . . . . . . . . . . . . . . . . 129

7 Investigation of the yz-plane 135

7.1 Experimental set-up . . . . . . . . . . . . . . . . . . 135

7.2 Statistical properties of the log-law region . . . . . . . . . . . . 138

7.2.1 Spatial correlations with $\Delta x=0 \ldots \ldots \ldots \ldots 141$

7.2.2 Spatial cross-correlations with $\Delta x=0 \ldots \ldots \ldots . \ldots 143$

7.3 Spatio-temporal correlations with $\Delta x=0 \ldots \ldots \ldots \ldots \ldots$

7.4 Spatio-temporal correlations with $\Delta x \neq 0 \ldots \ldots \ldots \ldots \ldots$

7.5 Properties of coherent velocity structures . . . . . . . . . . . . . 155

7.5.1 Loop-shaped structures . . . . . . . . . . . . . . . . . 155

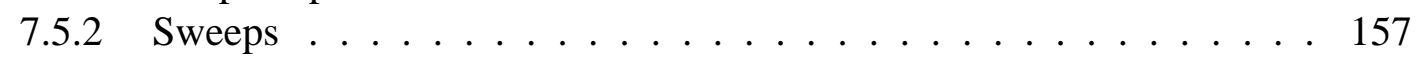

7.5 .3 Stream-wise vortices . . . . . . . . . . . . . . 160

8 Summary 163

$\begin{array}{ll}\text { Bibliography } & 169\end{array}$

$\begin{array}{ll}\text { Commonly used symbols } & 177\end{array}$

$\begin{array}{lr}\text { Index } & 180\end{array}$ 


\section{Introduction}

One of the fascinating phenomena of natural science is the turbulent state of the macroscopic flow motion in fluid-mechanics. Beside the omnipresent variety and beauty of the turbulent motion, the inherent fascination and attraction is strongly connected with the enormous difficulties associated with a mathematical and physical understanding. The principal difficulty, with respect to an inviscid fluid or a classical gas of point particles in equilibrium, results from the strong non-linearity in the conservation equation and the dissipative character e.g. the flux of energy from the large scales or eddies into progressively smaller and smaller ones. As a general mathematical solution of non-linear, non-equilibrium systems is out of reach from the present point of view, the properties of idealised flows with simple geometries are examined experimentally and numerically. Of primary interest is the two-dimensional turbulent boundary layer flow of an incompressible fluid along a flat plate with zero pressure gradient, because this flow reveals simultaneously two characteristic phenomena of turbulence, namely the effects of near-wall turbulence and the effects of intermittency, e.g the interaction of the turbulent boundary layer with the laminar outer flow according to figure 1.1. This particular flow evolves from a laminar boundary layer flow when the Reynolds number $R e_{x}=U_{\infty} x / \nu$ is sufficiently high. In this case flow disturbances with a particular wavelength grow, become unstable and share the energy from the mean motion over the degrees of freedom by non-linear interaction.

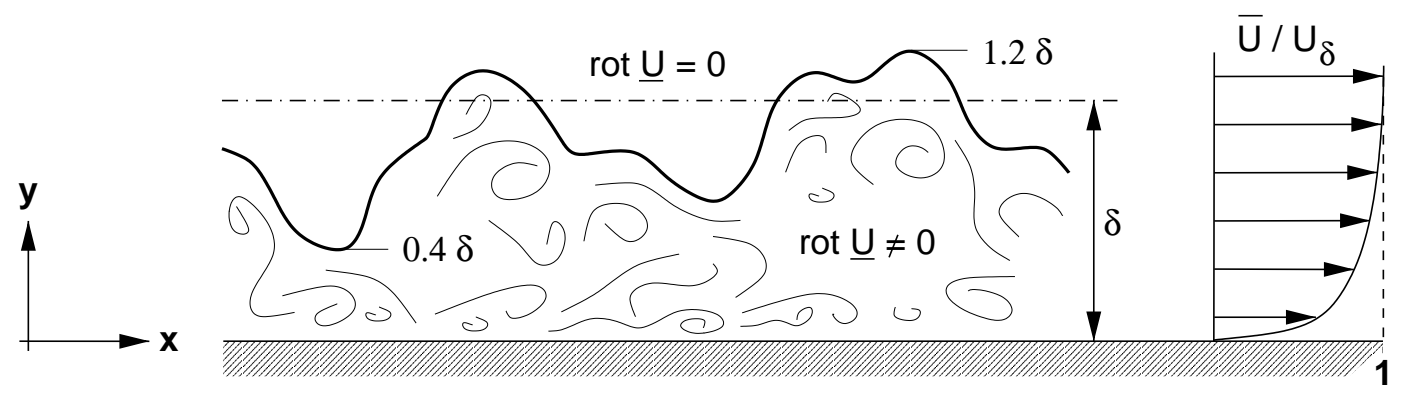

FIGURE 1.1: Instantaneous structure of a turbulent boundary layer and mean velocity profile after [37]

Beginning with the early channel and pipe flow measurements, published by Laufer [68, 69], and the zero pressure gradient boundary layer benchmark investigations along a flat plate by Klebanoff [55], turbulent boundary layers have been examined extensively because of their technological importance, their significance for the development of fundamental turbulence models and for the validation of numerical flow simulations $[89,96]$. The bulk of the quantitative investigations has been performed with intrusive single-point measurement techniques [21] such as pressure probes and hot-wire anemometer, but also non-intrusive flow visualisations techniques have been frequently applied to examine qualitatively the global features of 


\section{Introduction}

the flow $[15,24,25,28,54,57,58,59,79,81,84,92,103,110]$. Although the conclusiveness of these visualisations is often questionable, because of the complexity of the turbulent motion and inherent problems associated with the interpretations of the flow visualisation results as discussed by Hama [26], these investigations have improved the understanding of turbulence to a large extent, because it was possible to detect coherent flow structures such as low-speed streaks, shear-layers, stream-wise vortices and loop-shaped structures and to illustrate their significance for the turbulent mixing in wall-normal direction. In the following years, a number of partially contradicting vortical models have been proposed, designated as hairpin, horseshoe or lambda vortices in the literature [83, 98, 99], to link the coherent structures and processes identified as illustrated in figure 1.2 and figure 1.3 for example.

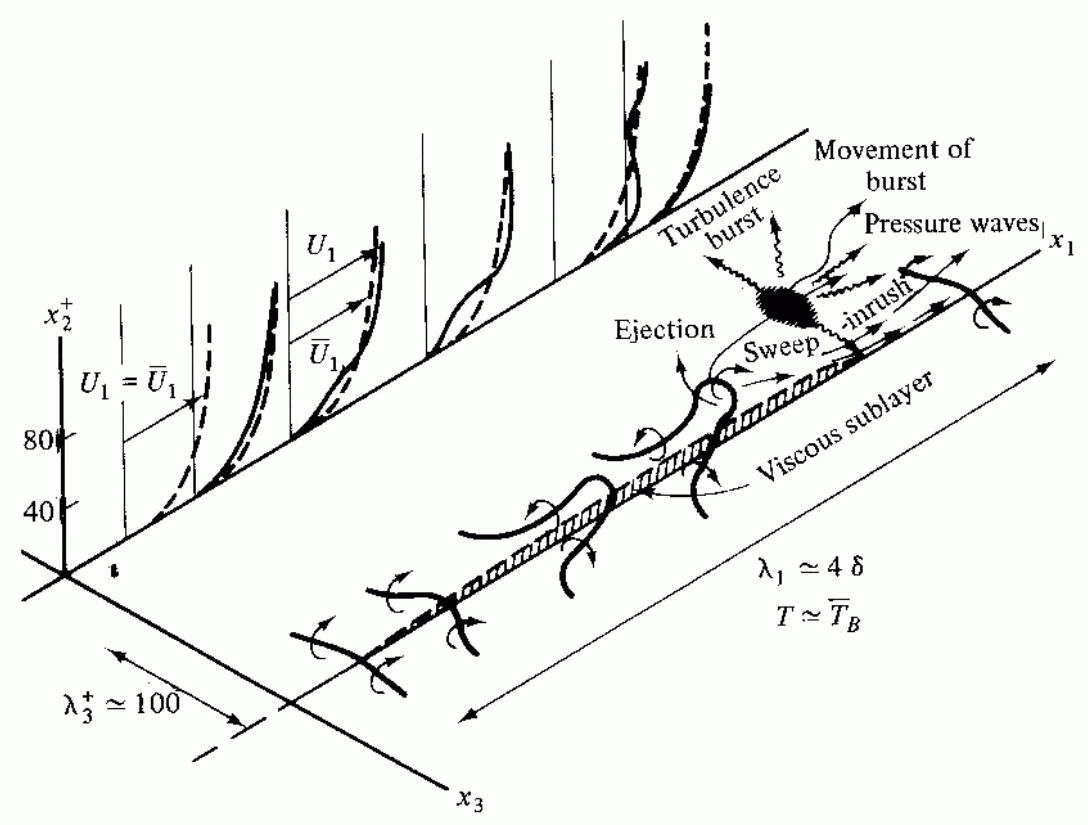

FIGURE 1.2: Formation of a hairpin vortex in a boundary layer from a span-wise vortex and decay of the vortex structure, after [31].

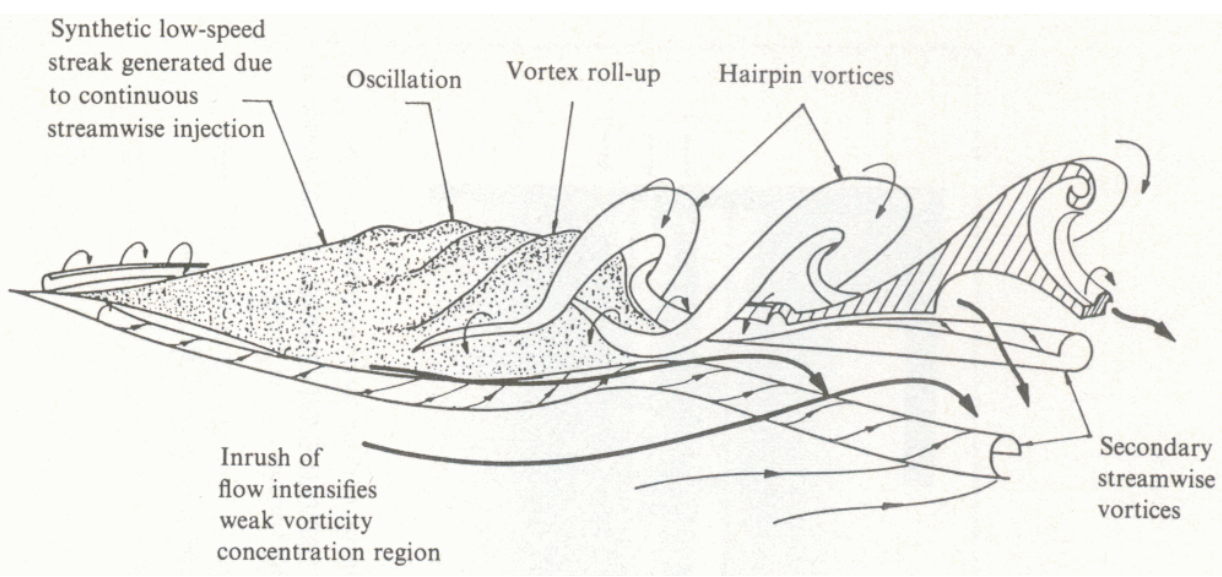

FIGURE 1.3: Schematic of break-up of a synthetic low-speed streak generating hairpin vortices. Secondary stream-wise vortical structures are generated owing to inrush of fluid, after [1]. 
As order can be always observed when the convection term in the equation of motion is dominant over the production, diffusion and dissipation term, there is no doubt about the existence of organised flow structures. However, their exact geometrical and kinematical properties are widely unknown and there is still a general controversy about which structures are fundamental and which ones are only secondary, which ones are dominant and which ones are irrelevant $[73,86,87,94]$. Based on the technological progress in laser, camera and computer technology in the last decade of the 20th century, it became possible to link the qualitative visualisations with the statistical results from the quantitative single-point measurements by applying non-intrusive optical multi-point measurement techniques such as the conventional and stereoscopic particle image velocimety, see $[4,38,44,74]$ for example, and it was partially possible to prove the validity of the proposed vortical models. However, due to the measurement noise and the limited spatial resolution, storage capacity and computer power at that time, only the properties of the large-scale structure could be deduced from a small number of samples. The information about the geometrical and kinematical properties of the small-scale structures on the other hand is still uncertain and also a consistent physical picture, which links the basic processes and explains their importance with regard to the turbulent transport is missing [31, 76, 86, 89, 94]. There is no doubt that further sophisticated experimental or numerical investigations are required to solve these questions and to add another piece to the puzzle of near-wall turbulence. As a direct numerical integration of the conservation equations is still out of reach, especially at high Reynolds numbers, it is generally believed that deeper insight strongly relies on the capabilities of advanced measurement techniques. To overcome the limitations of the existing measuring and visualisation techniques applied in fluid mechanics a stereo-scopic particle image velocimetry based measurement system was developed and applied for the investigation of the phenomena of near-wall turbulence. By using this technique it is possible to determine at any flow velocity all three velocity components in spatially-separated planes simultaneously or separated in time. It will be shown in the first part of this thesis that this technique is very reliable, robust and well suited for measuring with high accuracy and spatial resolution a variety of fundamentally important fluid-mechanical quantities, which are at present not available by any other known technique. In the second part of the thesis the experimental results obtained with this technique in a turbulent boundary layer flow will be outlined in order to answer some of the previously mentioned questions about the geometrical and kinematical properties of the coherent flow structures and their relation relative to the proposed vortex models.

Beside the difficulties associated with the measurement techniques nearly all experimental boundary layer investigations reported in the literature are faced with the problem that the generation of a fully-developed, turbulent boundary layer flow of large extent and range of scales is difficult to achieve due to the limited wind-tunnel dimensions. By using tripping devices right behind the leading edge of a flat plate, such as sand paper, wires or ribbon elements for example, a well-defined transition to turbulence can be reached, accompanied by a strongly-growing boundary layer thickness $\delta$ and range of scales. However, these devices introduce artificial flow-structures which alter the natural turbulence level and flow structure in the wake $[21,83,111]$. Without tripping the boundary layer, only natural flow structures are developing, but due to the gradual, non-uniform transition process, the onset of turbulence is delayed and strongly varies in time and spatial location. As a consequence, quantities like the boundary layer thickness $\delta$ are not well defined and the range of scales is rather small relative to the disturbed flow. In addition, a direct interaction between the outer region $(y>0.2 \delta)$ of 


\section{Introduction}

the turbulent boundary layer with the near-wall flow domain can take place and this may alter the structure of the flow in between both regions [41]. To overcome these principal difficulties associated with the preparation of a well defined flow, which corresponds to the idealised flow described by the equations of motion, the experimental investigation outlined in chapter 5 to 7 was prepared and performed in the temperature-stabilised, closed circuit wind tunnel at the Laboratoire de Mécanique de Lille (LML) shown in figure 1.4. This facility allows high resolution experiments at Reynolds numbers up to $R e_{\theta}=20000$ at free-stream velocities of $U_{\infty}=10 \mathrm{~m} / \mathrm{s}$. This is possible due to the remarkable boundary-layer thickness of $\delta>0.3 \mathrm{~m}$ at the end of the $20.6 \mathrm{~m}$ long, $2 \mathrm{~m}$ wide and $1 \mathrm{~m}$ high test section. The contraction ratio of the facility is 5.4 , the flatness of the wall is $1 \mathrm{~mm}$ on $5 \mathrm{~m}$ and the temperature of the flow can be stabilised with an uncertainty of $\pm 0.2^{\circ}$. The measurements were taken $18 \mathrm{~m}$ from the testsection inlet at free stream velocities of $3 \mathrm{~m} / \mathrm{s}$ and $7 \mathrm{~m} / \mathrm{s}$. At this location, the Reynolds number based on the momentum thickness $\theta$ is $R e_{\theta}=7800$ and 15000. For further details about the wind-tunnel and flow characteristics, like shape parameter $H_{12}$, skin friction coefficient $c_{f}$ and strength of the wake component $\Delta\left(\bar{U} / u_{\tau}\right)$, see [12].
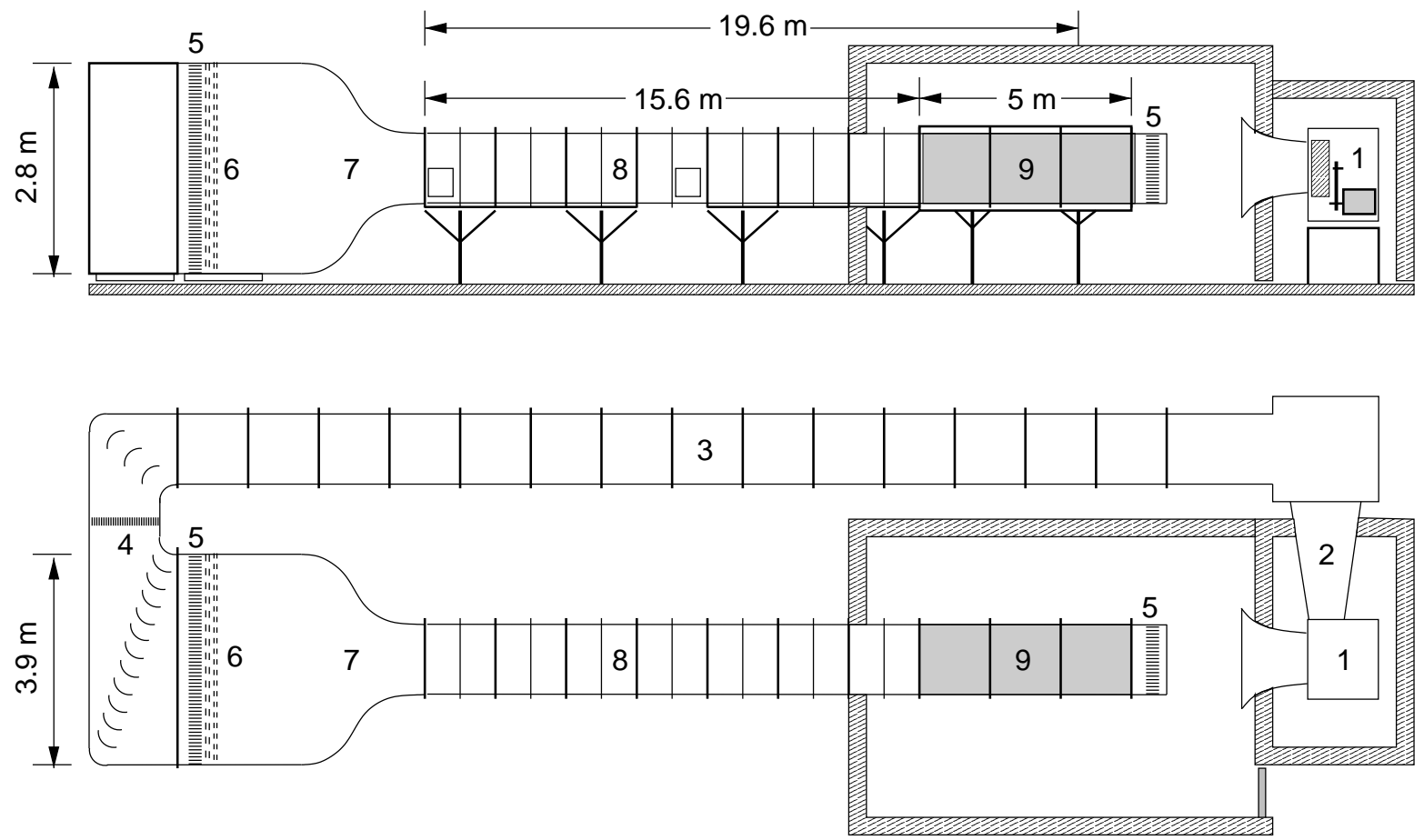

FIGURE 1.4: Top and side view of the temperature-stabilised closed circuit boundary layer wind tunnel at the Laboratoire de Mécanique de Lille (LML) in France. 1 engine ( $37 \mathrm{~kW}$ ) with fan, 2 diffuser, 3 return circuit, 4 heat exchanger, 5 honeycomb, 6 screens, 7 settling chamber with nozzle, 8 turbulent boundary layer development zone, 9 transparent testing zone.

\section{Organisation of the thesis}

This thesis is composed of six mostly self contained main chapters that can be read independently without needing continuous cross referring.

Chapter 2 examines various aspects of the Particle Image Velocimetry (PIV) which are of fundamental importance for accurate measurements, especially in turbulent flows, but which 
were not considered in detail in the literature [2, 29, 85, 104]. Starting point is the basic problem associated with the reproducible generation of sufficiently monodisperse liquid particles with an appropriate size, shape and density such that they follow the macroscopic flow motion faithfully without disturbing the flow or fluid properties. In the past, very little attention has been paid to the observation that the particle size distribution generated may strongly deviate from the desired distribution if the liquid level in the aerosol generator changes, the pressure varies, or the nozzle holes are contaminated for any reason. This may cause serious problems as the accuracy of all tracer-based velocity measurement techniques, such as laser Doppler anemometry (LDA), particle image velocimetry (PIV) or Doppler global velocimetry (DGV), is ultimately determined by the particle dynamics [3]. Beside the fundamental problems associated with the generation of appropriate tracer particles, the registration, the storage and the read-out of the particle images will be investigated in detail in section 2.3. This process is another key element in PIV as the accuracy of the technique strongly depends on the precision with which the image displacement can be related to particle locations and their respective particle displacements [3]. Parts of the results have been already published in [39, 42, 47].

Chapter 3 focuses on the stereoscopic PIV techniques because the conventional PIV system outlined in chapter 2 is not well suited to examine turbulent flows for two reasons. First, only the projection of the three-dimensional flow velocity inside the measurement plane can be determined with the conventional PIV technique. Second, the two velocity components that can be measured are biased when the flow is three-dimensional because of the perspective error. In section 3.1 the advantages and drawbacks of various stereoscopic recording configurations will be compared and the basic principles of different evaluation schemes known as image warping, vector field warping and interrogation window warping will be discussed in section 3.2. Of primary interest is the investigation of the different image interpolation schemes which are required for the analysis of the image pairs, because the measurement error depends strongly on the interpolation algorithm applied. Another systematic measurement error that was discovered by applying the stereoscopic PIV is investigated in section 3.3. As this error can be large relative to all other possible measurement errors a calibration validation technique is examined which has been developed to detect and compensate this error completely. The general material of this chapter is mainly based on $[40,42]$ and the section about the calibration validation method and the different evaluation schemes can be found partially in $[39,44]$.

Chapter 4 describes the principles and properties of the multiplane stereo PIV technique. This technique was developed to obtain with high accuracy and spatial resolution information about the spatio-temporal flow unsteadiness and to determine fundamentally important fluid-mechanical quantities which are presently not accessible with any other known measurement technique technique. First of all the principles and properties of the techniques will be outlined along with the required four pulse laser system. Thereafter, the synchronisation and alignment of the system component will be discussed and different modes of operation will be outlined which have been applied for the fluid-mechanical investigations in chapter 6 and 7. In section 4.6 and 4.7 polarisation effects and the effect of optical aberrations on the measurement precision will be studied. In particular, it will be shown how to reduce undesirable optical aberrations below the resolution of the recording medium and how to eliminate certain aberrations completely by accepting aberrations of other types which are of no harm in PIV. To examine the performance and reliability of the multiplane stereo PIV technique, the acoustic receptivity of a laminar boundary layer along a flat plate with zero pressure gradient 


\section{Introduction}

is examined in section 4.8. This experiment was performed in the low-turbulence wind-tunnel at DLR Göttingen. The general idea of the measurement technique, possible realisations and different applications have been already published by the author [41, 39, 45, 46, 49].

Chapter 5 shows the main fluid-mechanical results measured in the stream-wise wallnormal plane ( $x y$-plane) of the turbulent boundary layer at $R e_{\theta}=7800$ and 15000 with the stereoscopic PIV technique outlined in chapter 3. The measurement was performed $18 \mathrm{~m}$ downstream of the test-section inlet in the temperature-stabilised wind-tunnel shown in figure 1.4. In section 5.1 the basic theoretical concepts required for a statistical description of turbulence are summarised and the details of the experimental configuration and the recording parameter are outlined in section 5.2. To validate the flow characteristics and the performance and accuracy of the PIV system applied, in section 5.3.1 the primary statistical properties of the turbulent boundary-layer flow are compared with the theoretical predictions outlined in section 5.1, as well as the experimental results reported in the literature [21]. Thereafter, the properties of the normalised spatial correlation and cross-correlation functions are examined to link the statistical results with the concept of coherent flow structures. This concept assumes that the complexity of the turbulent motion is simply a result of the presence and interaction of relatively simple vortical structures or eddies. The average size and shape of the dominant coherent flow structures is deduced from the primary components of the double correlation tensor and the dimensions of the flow structures being responsible for the production and transport of turbulence is determined from the various cross- and conditional-correlations. Because of the fact that the coherent flow structures have a history of development while they are transported downstream, the spatial correlation functions include realisations of a large number of structures at various stages of their life history. To examine the properties of the individual coherent flow structures, such as their significance for the production of turbulence, their mutual interaction and finally their relation with respect to idealised vortex models mentioned on page 6, typical instantaneous velocity fields are finally analysed in section 5.4.

Chapter 6 examines the results measured with the multiplane stereo PIV technique outlined in chapter 4 in stream-wise span-wise planes ( $x z$-planes) at $y^{+}=10,20,30$. These planes are of particular interest because of the strong dynamic of the turbulent fluctuations in this domain according to the results in chapter 5. The intensity of the different flow structures and their propagation direction relative to the wall are examined in section 6.2.1 and the size, shape and intensity of various spatial correlation, cross-correlation and conditionalcorrelation functions are analysed in section 6.2.2 and 6.2.3 in order to obtain quantitative information about the structural features of the coherent flow structures and their significance for the turbulent mixing. In section 6.3, the mean convection velocity of the different coherent velocity structures present in the near-wall region of the turbulent boundary layer is estimated from the spatio-temporal structure of the correlation function by using various methods and the dominant coherent flow structures being present in the near wall region are investigated in detail in section 6.4. Of primary interest is again their size and shape, their significance for the production of turbulence and their mutual interaction. In the first part of the investigation flow structures will be considered which can be labelled as dominant due to their occurrence. Thereafter, flow structures are examined that can be characterised as dominant due to their large amplitude in the instantaneous Reynolds shear stress component $-\rho u v$. Although these structures do not appear frequently, they reveal general features that can be often observed in the turbulent flow even when the peak intensity of the Reynolds shear stress is lower. In particular it will be proven on the basis of the concept of frozen pattern, which states that a spatially 
varying signal can be transformed into a time dependent signal at a fixed point, whether a characteristic velocity pattern, that was observed in the past by using hot-wire probes, is related to the motion of particular coherent structures. The results of this chapter are partially published in [41].

Chapter 7 focuses finally on the wall-normal span-wise plane ( $y z$-plane) investigation of the turbulent boundary layer by means of multiplane stereo PIV at $R e_{\theta}=7800$ and 15000 . These investigations were mainly performed to examine the validity of the frozen pattern concept, applied in chapter 6 , and to prove the existence of stream-wise vortices in the nearwall region and their significance for the turbulent mixing normal to the wall. Two independent experiments are described with different spatial separation between the measurement planes in stream-wise direction $(\Delta x=0$ and $\Delta x \neq 0)$ and various temporal delays between a pair of measurements. Due to the widespread opinion that reliable experimental results are very difficult to achieve when the orientation of the light-sheet is perpendicular to the main flow direction, in section 7.2 the statistical results are validated against the results presented in the chapter 5. In section 7.2.2 the structural features of the coherent flow structures are displayed and the model assumption made for the interpretations of the results presented in chapter 5 and 6 will be examined. In section 7.4 the decay of the coherent structures with increasing time is investigated as a function of the wall distance to validate the frozen pattern hypothesis. In section 7.5 the existence and relevance of the stream-wise vortex pairs for the turbulent mixing in wall bounded flows is finally examined and the concept of hairpin or horseshoes like structures is discussed as well as their connection with shear-layers and streaks. Parts of this chapter are already published in [49]. As the fully-developed turbulent boundary layer flow along a flat plate is considered as one of the basic academic flows which is accessible by Direct Numerical Simulations (DNS), and a test case for the validation of turbulence models required for Large Eddy (LES) and Reynolds Averaged Navier-Stokes Simulations (RANS), the statistical results presented in chapter 5 to 7 are displayed in various representations to simplify a comparison in the future. 
1 Introduction 


\section{Particle Image Velocimetry}

The tracking of individual objects in space and time by means of optical techniques has a long history and a lot of valuable information could be collected in the past. By combining these observations with the human enthusiasm, creativity and intellect, an understanding of nature and science could be developed from the early days of conscious reflection to the present state. The reader may remember that, based on the precise observations of the planetary motion by Tycho Brahe (1546-1601), Johannes Kepler (1571-1630) could reduce the complexity of the ancient Greek epicycle theories, developed by Claudius Ptolemaios (100-170) and others, to three simple laws, which describe the motion of the planets around the sun and with respect to each other, and he could explain the varying size and brightness of the planets in time, for example. Seven decades later, Isaac Newton (1643-1727) developed an idea to explain these laws and all observed deviations from the ideal motion from first principles, by introducing the concept of force between any pair of objects, and he could link the planetary motions with the laws of motion from Galileo Galilei (1564-1642). The precision and beauty of Newton's axiomatic theory was so convincing that the vision of their absolute certainty could be preserved for more than three centuries. The deciding factors leading to this success were the absence of friction within the empty space, the simple force law between the objects and, finally, the fact that the sun could be considered as motionless due to its enormous mass with respect to the other planets. Unfortunately, the physics of continua is much more complex than point mechanics, as each single fluid element interacts with its whole environment in a complex manner and the effect of friction can only be neglected under restricted conditions. As a consequence, the motion of fluid elements is strongly correlated and its state at a single point requires information about the local translation, rotation and deformation of each fluid element. This implies that fluid-mechanical considerations always require a detailed knowledge of the spatial distribution of the velocity and its spatio-temporal variations. Single probe techniques as they are generally employed in fluid mechanical research provide only local information at one single point but with high temporal sampling rate. The Particle Image Velocimetry (PIV) in contrast yields in general no reliable temporal information (at least in its present development state) but the desired global information can be obtained with a high accuracy and spatial resolution as described in the following sections.

\subsection{Principles}

The Particle Image Velocimetry is a well established, non intrusive technique for measuring the spatial distribution of the velocity within a single plane inside the flow, indirectly via the displacement of moving particles groups within a certain time, see figure 2.1 and [85]. For this purpose the flow region under consideration is homogeneously seeded with appropriate tracerparticles such that their injection and presence does not affect the flow or fluid properties. The 


\section{Particle Image Velocimetry}

concentration of the particles must be well adjusted with regard to the finest flow structures, in order to sample the flow properly, and the deviation of the particle velocity $\underline{u}_{\mathrm{p}}$ from the real flow motion $\underline{U}$ must be negligible compared to the uncertainty of the imaging and recording system and to the uncertainty due to the evaluation procedure. In this case the difference between the following expressions is negligible.

$$
\int_{t}^{t+\Delta t} U\left(t^{\prime}\right) d t^{\prime} \approx \int_{t}^{t+\Delta t} u_{\mathrm{p}}\left(t^{\prime}\right) d t^{\prime}
$$

After the required seeding concentration has been obtained, a desired plane inside the flow is illuminated twice by a thin laser light-sheet. The light scattered by the tracer-particles at time $t$ and $t^{\prime}$ in the direction of the recording optics is usually stored on individual frames of a single frame transfer CCD camera, whose optical axis is perpendicular to the light-sheet. It is obvious that the fluid must be optically accessible and sufficiently transparent for the wavelength under consideration. Furthermore, the light-sheet intensity and field of view must be well balanced to the scattering properties of the particles, the performance of the optical system and the sensitivity of the camera.

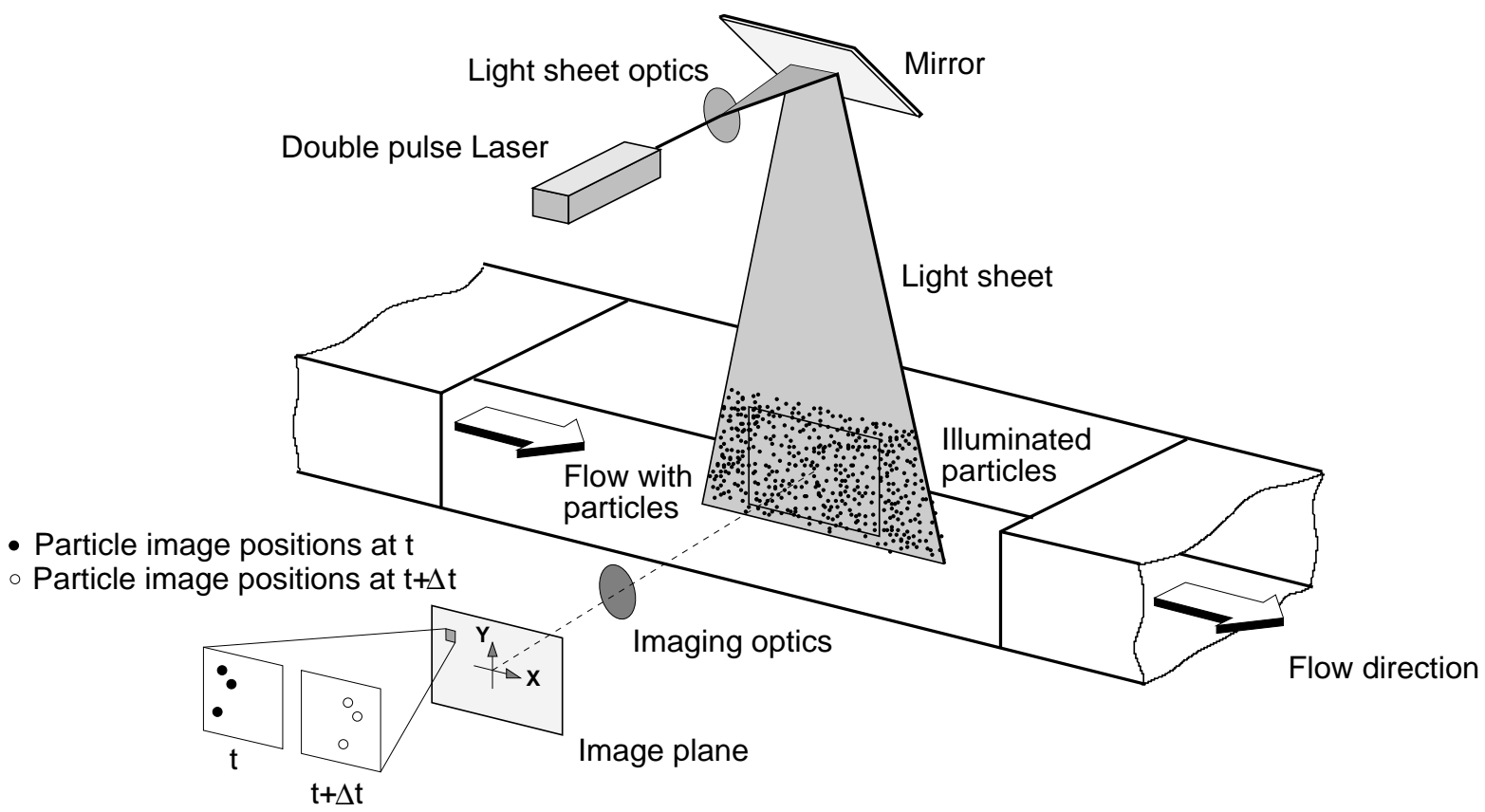

FIGURE 2.1: Schematic set-up of particle image velocimetry system after [85]. A desired plane inside the flow is illuminated twice by a thin laser light-sheet and the scattered light emerging from the homogeneously distributed particles in the direction of the imaging optics is recorded.

The local in-plane particle image displacement component $\Delta X$ of the double exposed particles is finally determined from the two single exposed recordings by means of spatial cross-correlation techniques and afterwards divided by $\Delta t$ and the magnification factor $M$ of the imaging system to calculate the first order approximation of the velocity field according to the following equation.

$$
\int_{t}^{t+\Delta t} u_{\mathrm{p}}\left(t^{\prime}\right) d t^{\prime} \approx x(t+\Delta t)-x(t)=\Delta x \rightarrow \frac{\Delta x}{\Delta t}=\frac{\Delta X}{M \Delta t} \approx U
$$


It is of fundamental importance to realize that the particle displacement must be small relative to the finest flow scales, as only phenomena that occur over a time interval which is longer than $\Delta t=t^{\prime}-t$ and that have a spatial extent larger than the absolute displacement $\Delta x$ can be resolved, but the particle image displacement $\Delta X$, on the other hand, must be large for accurate measurements.

This brief overview already implies that the complexity of the technique arises basically from the technical components involved and their mutual dependence on each other and less on the principles of the technique itself. In terms of accuracy, for example, the particles should be sufficiently small and their density should exactly match the density of the surrounding fluid. Unfortunately, this is not often feasible for a desired field of view and a given laser power, light sheet thickness, transparency of the fluid, imaging optics and sensitivity of the digital camera, as the scattering intensity decreases rapidly with decreasing particle diameter as shown in figure 2.2. Decreasing the light-sheet width or thickness may partially help but
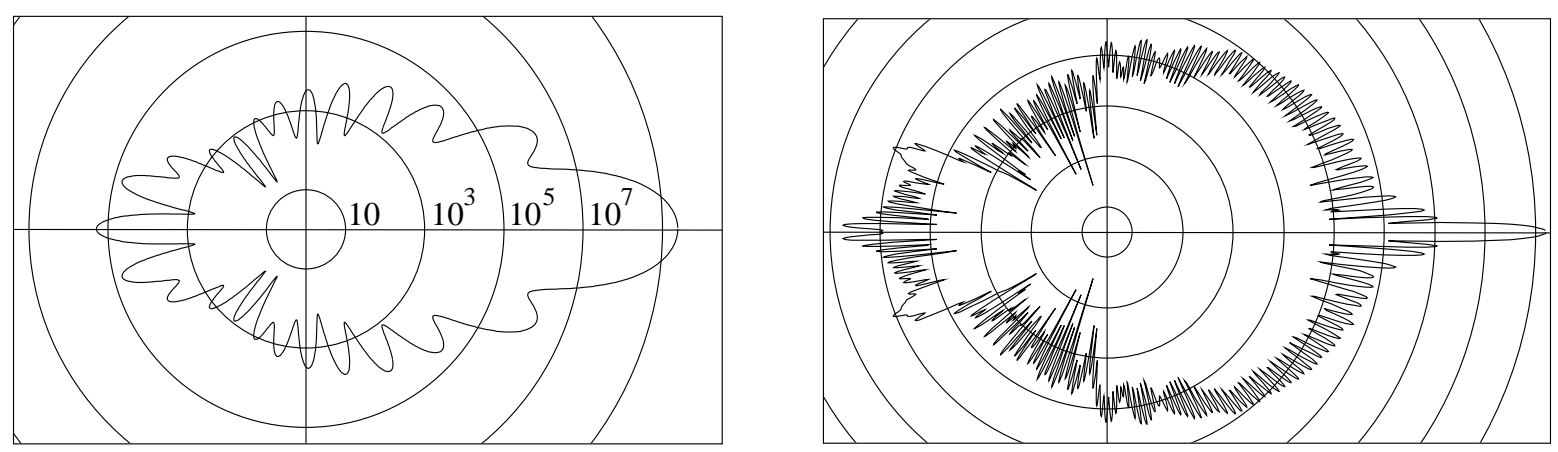

FIGURE 2.2: Light intensity scattered by spherical oil particles of different size in air (left: $d_{\mathrm{p}}=$ $1 \mu \mathrm{m}$, right: $d_{\mathrm{p}}=10 \mu \mathrm{m}$ ), illuminated from left with a plane monochromatic wave front, after [85]. The complex spatial intensity distribution, with a maximum in forward direction, results from the interference between the reflected, refracted and diffracted wave front.

the size of the largest resolvable scales will decrease as well and the three dimensionality of the flow may cause further problems as will be explained later. To use a powerful laser seems to be the appropriate solution but beside the costs, strong reflections from model surfaces or undesirable disturbances of the flow due to acoustic excitation or thermal response of the flow have to be taken into account [48]. An intensified camera could be applied as well but a reduced spatial resolution and an increased noise level must be accepted. Alternatively, the evaluation procedure could be adapted but then the accuracy of the velocity estimation and the validity of the well established principles may become questionable. Furthermore, it cannot be recommended to analyse data where the desired fluid mechanical information, hidden in the particle image displacement, cannot be uniquely determined. So increasing the particle size may be the appropriate solution at the end, but how this can be achieved is unknown. Anyway, in order to make the right decision while setting up and aligning the experiment, a clear understanding of the basic components is essential. For this reason the production of desired particles, the problems associated with the recording of the particle images and the determination of the image displacement will be treated in chapter 2 . In chapter 3 and 4 the more sophisticated recording techniques are considered which have been applied for the fluid mechanical investigations presented in the second part of the thesis. 


\subsection{Generation of appropriate tracer-particles}

The starting point and basic problem associated with all tracer-based optical velocity measurement techniques in fluid mechanics is the reproducible generation of sufficiently monodisperse particles with an appropriate size, shape and density such that they follow the macroscopic flow motion faithfully without disturbing the flow or fluid properties. In the past, smoke generators, which deliver condensed oil vapour, or air-operated aerosol atomisers, have been widely and successfully used as these devices easily produce the particle concentrations required for high resolution measurements in large wind tunnels [75]. Unfortunately, very little attention has been paid to the observation that the particle size distribution generated may strongly deviate from the desired distribution if the liquid level in the generator changes, the pressure varies, or the nozzle holes are contaminated for any reason [17, 47]. This may cause serious problems, especially for investigations with strong vortices or transonic flows with shocks, as the accuracy of all tracer-based velocity measurement techniques, such as laser Doppler anemometry (LDA), particle image velocimetry (PIV) or Doppler global velocimetry (DGV), is ultimately determined by the particle dynamics. In the following a systematic analysis of the control parameters influencing the particle size distribution will be presented for different types and realizations of atomiser nozzles.

\subsubsection{Description of the experiment}

To examine the conditions and physical processes leading to a desired particle distribution, a fully transparent perspex generator, $150 \mathrm{~mm}$ in diameter and $280 \mathrm{~mm}$ high, was built with a detachable lid containing two orifices: One $8 \mathrm{~mm}$ pressurised air inlet in the centre with a fine thread for easy nozzle mount and a $11 \mathrm{~mm}$ aerosol outlet [47]. For the experimental investigation three different types of nozzles have been considered, see figure 2.3. The first one, called a reference nozzle in the following, consists of a 220-mm-long brass tube with an internal diameter of $5 \mathrm{~mm}$ and four crosswise arranged holes, each $1 \mathrm{~mm}$ in diameter and $20 \mathrm{~mm}$ forward from the closed end of the tube. The holes are located $80 \mathrm{~mm}$ below the liquid level and well above the generator bottom in order to avoid free surface effects during the mixing process and a bias due to the presence of the solid boundary. The second nozzle under consideration was a Laskin nozzle with the same geometrical dimensions as the reference nozzle but with a 6-mm-thick and 18-mm-wide brass disk immediately above the nozzle exits with $1 \mathrm{~mm}$ liquid feed holes on top of the tube holes [67]. It is assumed in the literature that, in operation, four symmetrically arranged air jets of identical mean velocity distribution emerge from the nozzle exits and produce submicron particles when the high velocity region of the jet interacts with the liquid provided by the feed holes. These particles are supposed to be embedded in bubbles and escape when the bubbles reach the liquid surface. In contrast to the other two nozzles, the third one sucks the liquid from below through a small capillary when compressed air is blown sideways through the outlet holes whose locations are above the liquid level [75]. It should be emphasised that this suction nozzle is not designed for the atomisation of glutinous liquids such as vegetable oil or Diethylhexylsebakat (DEHS), but as this nozzle type is increasingly applied in combination with both liquids, its performance will be analysed here as well. For the determination of the volumetric particle size distribution, a laser diffraction technique was applied which can resolve particle diameters down to $0.25 \mu \mathrm{m}$ by analysing the three-dimensional diffraction pattern of a particle ensemble according to ISO 

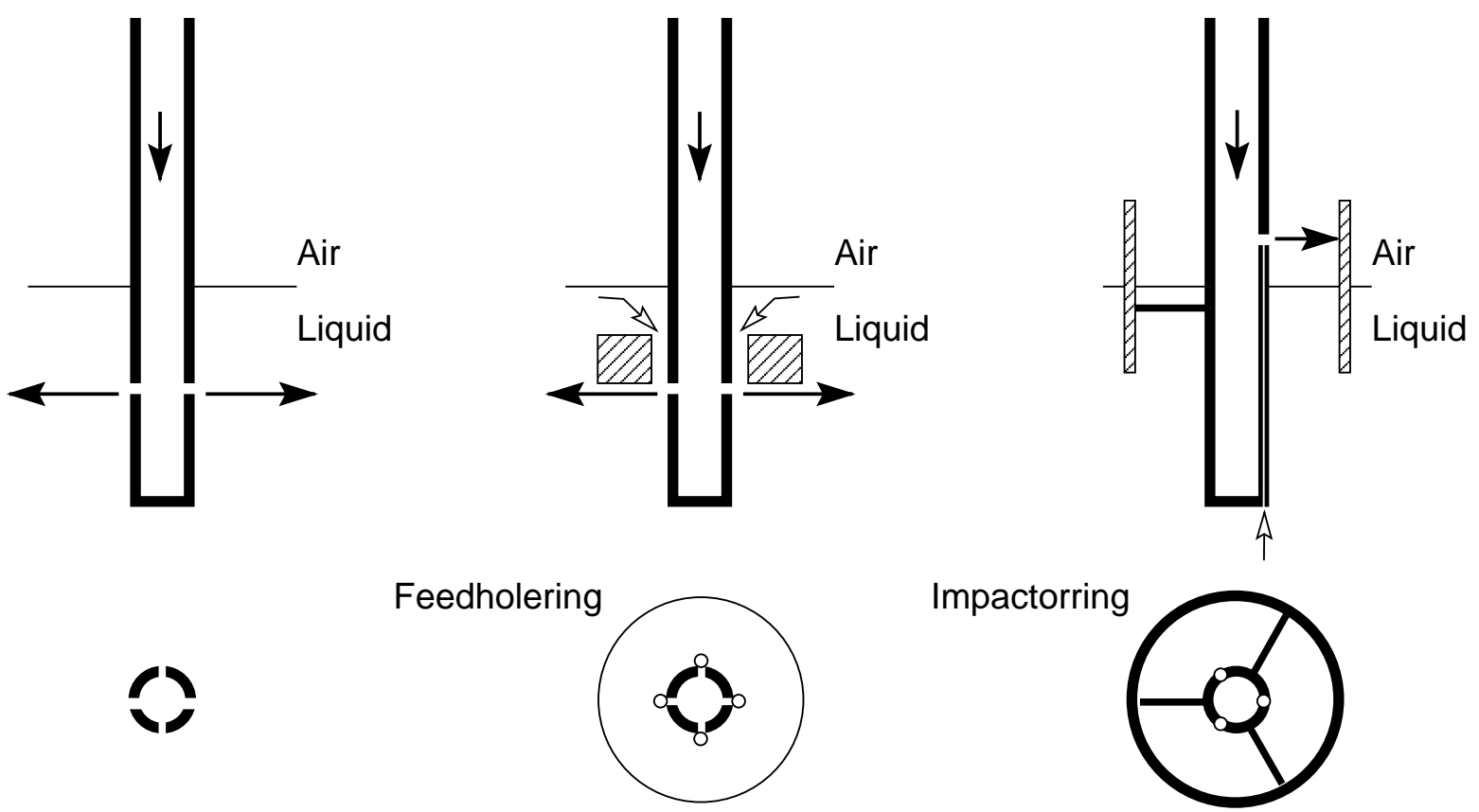

FIGURE 2.3: Principal side and top view of three utilised atomiser nozzles with different mechanisms for particle generation. From left to right: reference nozzle, Laskin nozzle and suction nozzle with impactor ring.

13320-1, see [34]. This is possible for spherical particles with known optical properties, when the particle concentration within the measurement domain is sufficiently low such that multiple scattering and optical interference between the scattered radiation from different particles can be neglected. Otherwise the measurements would be biased, as the diffraction pattern of a particle ensemble is no longer identical to the superposition of the individual scattering patterns of all particles present.

\subsubsection{Particle size analysis}

To ensure controlled boundary conditions before starting the experiment, each nozzle was cleaned and the symmetry of the emerging jets was analysed, as any asymmetry would indicate damages at the nozzle exit due to deposition of old oil or burr, for example. In addition, all particles produced during the adjustment of the desired pressure level were completely removed from the generator and the tube leading to the optical system. The reproducibility of the generated particle distribution was finally confirmed by performing four independent measurements for each set of parameters. To exclude the influence of physical properties of the liquid to be atomised, only vegetable oil with $\mu=0.026 \mathrm{~kg} /(\mathrm{ms})$ and $\rho_{\mathrm{p}}=874 \mathrm{~kg} / \mathrm{m}^{3}$ and DEHS with $\mu=0.023 \mathrm{~kg} /(\mathrm{ms})$ and $\rho_{\mathrm{p}}=912 \mathrm{~kg} / \mathrm{m}^{3}$ were used for all experiments, as both liquids behave very similarly.

Figure 2.4 reveals the volumetric particle size distribution for the described nozzles for two typical pressure states ( 0.5 and 1 bar) and, in addition, the distribution generated by means of a smoke generator has been added for comparison. First, it can be seen that the performance of the smoke generator is quite good with respect to the other atomisers, especially if the shape, bandwidth and upper limit of the particle size distribution are considered. Unfortunately, 

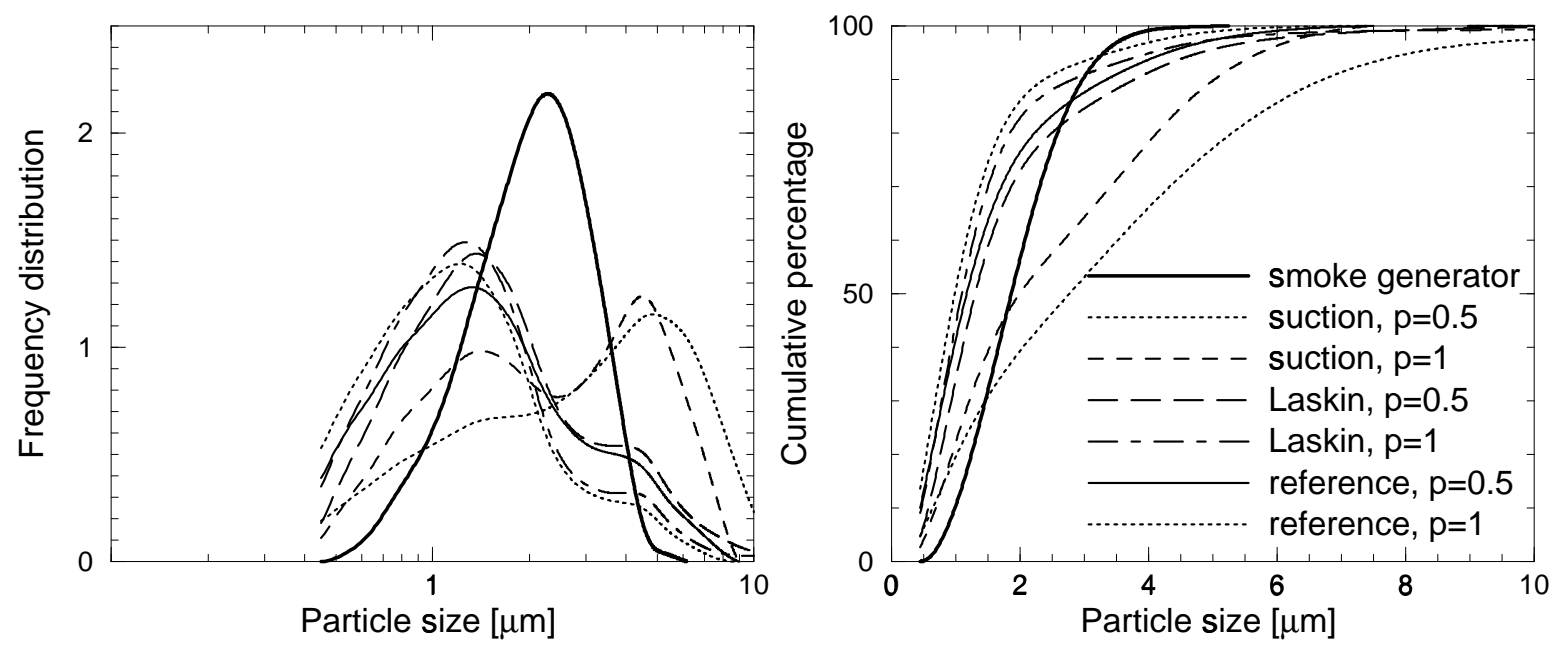

FIGURE 2.4: Volumetric particle distribution, according to ISO 13320-1, generated by three different atomiser nozzles for two pressure states and a smoke generator which delivers condensed oil vapour after evaporation by heating.

smoke generators are often not well suited for velocity measurements due to the fact that the particle concentration delivered by the generator is fixed, and the mean particle size can not be altered towards smaller particle diameters. However, this can be done by using nozzles as will be seen later. The second notable result in figure 2.4 is the similarity between the distributions generated by means of the reference and the Laskin nozzle. This is not surprising for two reasons: First, the influence of the ring above the Laskin nozzle is negligible, as only a minor part of the expanding and diverging air jet interacts with the ring. Secondly, it will be shown in the next section that the vertical suction holes do not feed the horizontal airstream with fluid, as assumed in the literature. This could be clearly proven for every pressure supplied by analysing the rising bubbles created at the top of the so-called liquid feed holes. The third remarkable result visible in the same figure is the strong dependence of the particle size distribution on the pressure. Whereas for the Laskin and reference nozzles only a small shift of the maximum towards $d_{\mathrm{p}}=1 \mu \mathrm{m}$ diameter and a decrease of the shoulder at $d_{\mathrm{p}}=4 \mu \mathrm{m}$ can be observed, the distribution of the suction nozzle develops from a single peak to a double peak distribution with a maximum at $d_{\mathrm{p}}=1.5 \mu \mathrm{m}$ and $d_{\mathrm{p}}=4 \mu \mathrm{m}$. The double peak distribution indicates that two different physical processes are probably involved in the production of the particles, and it seems likely that the process responsible for the generation of small particles requires higher pressure values. However, further experiments are required to validate this assumption. Finally, it should be noted that the distribution of the smoke generator analysed is quite monodisperse, compared with the distributions from the atomisers, but the reference nozzle produces the largest fraction of particles below $1 \mu \mathrm{m}$ followed by the Laskin and the suction nozzles. Based on these results, it was decided to examine only the reference nozzle in detail, as the Laskin and suction nozzles possess no advantage with respect to the reference nozzle when the volumetric particle size distribution and concentration are considered. However, this may become different for seeding materials whose viscosity deviates strongly from the fluids analysed here.

Figure 2.5 and 2.6 show the volumetric particle size distributions for the reference nozzle as a function of the hole diameter $d$, the external pressure $p$, the number of holes per nozzle 

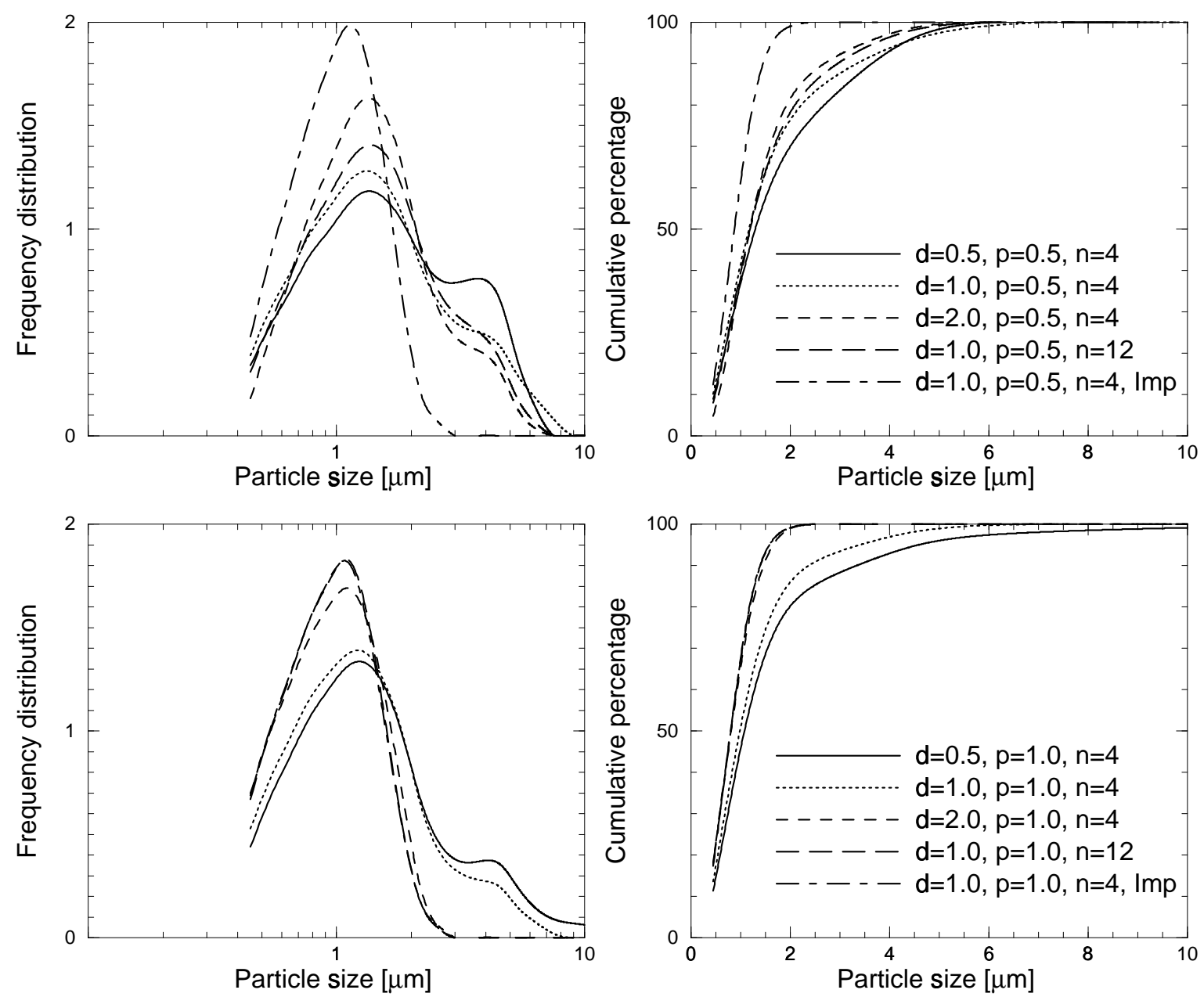

FIGURE 2.5: Volumetric particle size distribution for various hole diameters $(d=0.5,1,2 \mathrm{~mm})$, pressure ( $p=0.5$ and 1 bar), number of holes per nozzle ( $n=4$ and 12) and impactor.

$n$ and the effect of an impactor behind the output of the seeding generator. The upper left plot of figure 2.5 implies that shortly above the minimum pressure required for the production of particles, only the impactor is well suited to keep the particle size distribution sufficiently narrow and the mean particle diameter sufficiently small for accurate flow investigations, but it could be seen by visual inspection, that the particle concentration produced is quite low when only a single four-hole nozzle is used. The effect of the impactor can be clearly seen by comparing the dot-dashed line with the dotted graph. It should be mentioned that additional use of more impactors (cascade arrangement) has no further effect on the particle size distribution. By increasing the pressure from 0.5 to 1 bar, the exit velocity of the air jet reaches the speed of sound and the performance of the two high mass flux nozzles with $(d=2, n=4)$ and ( $d=1, n=12$ ) becomes comparable with the desired impactor distribution but the number of particles produced is several orders of magnitude higher as could be qualitatively seen by visual inspection. The other nozzles, in contrast, need much higher pressure before the shoulder disappears and the mean diameter becomes about $1 \mu \mathrm{m}$, see figure 2.6. This implies that a contaminated nozzle hole, due to polluted air or encrusted oil, will tend to create larger particles as the effective diameter decreases. This was validated in several experiments. Unfortunately, the high pressure investigation could not be performed by using the high flow rate nozzles, 

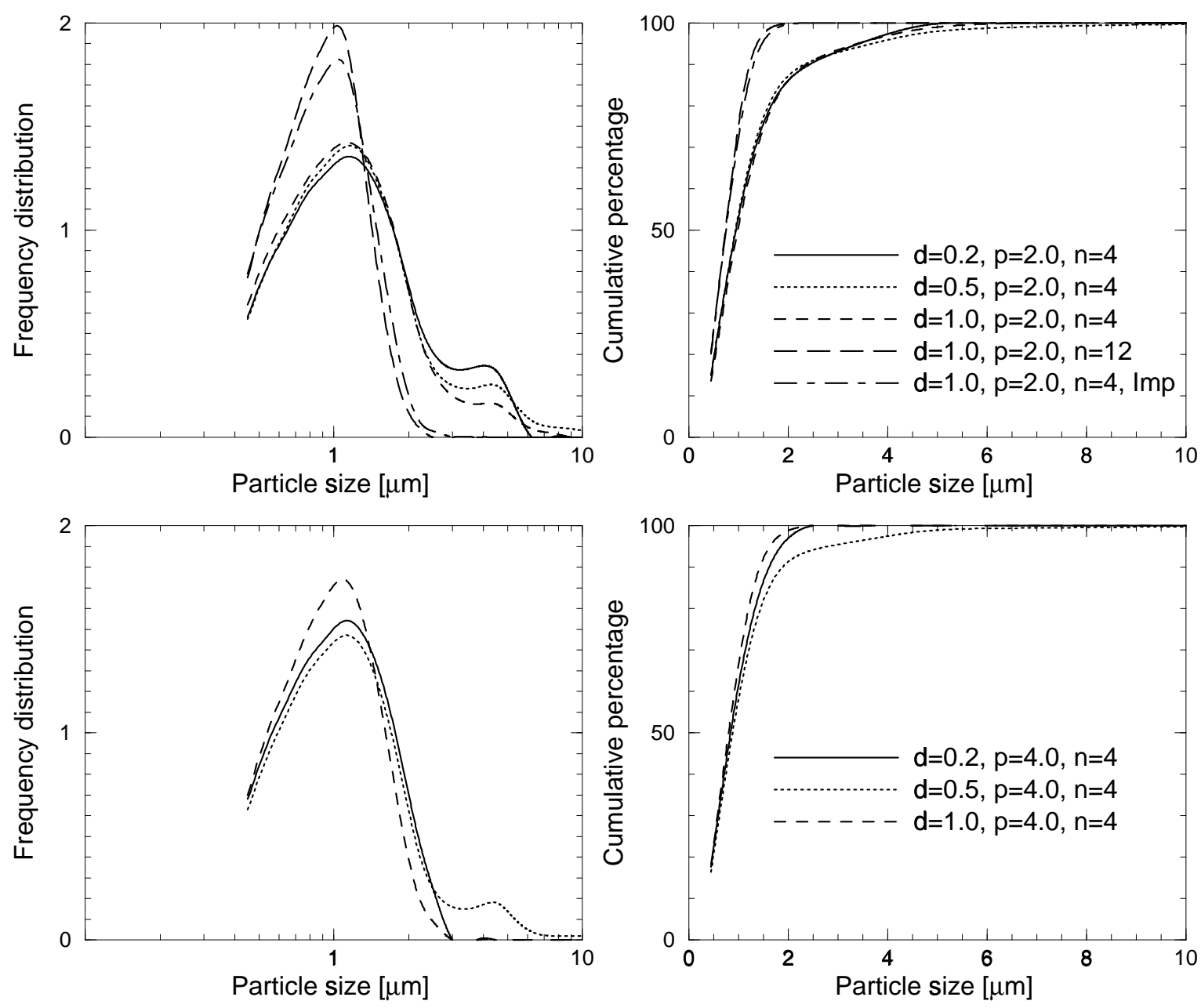

FIGURE 2.6: Volumetric particle size distribution for various hole diameters $(d=0.2,0.5,1 \mathrm{~mm})$, pressure ( $p=2$ and 4 bar), number of holes per nozzle ( $n=4$ and 12) and impactor.

as serious pollution of the optical system was likely, but a nozzle with a tiny hole diameter of $0.2 \mathrm{~mm}$ started to produce a sufficient amount of particles for reliable size measurements. The impactor result is also missing as the expanded air behind the nozzle could not be fed through the four impactor holes although their aperture area equals the area of the four nozzle holes. By increasing the impactor hole number, this difficulty could be solved, but this would reduce the performance of the impactor as the acceleration of the particles within the impactor decreases. Generally, it can be stated that the bandwidth and mean diameter of the distribution decrease to a final distribution if the diameter $d$, pressure $p$, and the number of holes $n$ are increased, or when an impactor is used alternatively. Thus, the determining factor seems to be the mass flux and less the geometrical properties of the nozzle itself. This is obvious as the particle size is three orders of magnitude smaller than the hole dimensions. Furthermore, it should be noted that the nozzles considered do not produce any particles larger than $10 \mu \mathrm{m}$. This allows the largest possible error in velocity measurement to be estimated for a particular flow.

The difficulty associated with this investigation is to understand why a four-hole nozzle with $1 \mathrm{~mm}$ drills produces different particle sizes under the same boundary conditions as the same nozzle with 12 identical holes, for example, arranged in such a way that the near fields 
of the jets do not interact with each other (three planes $20 \mathrm{~mm}$ apart from each other, each with four holes arranged crosswise). As only the flow rate (and thus kinetic energy) entering the liquid reservoir and available for turbulent mixing differs, it seems likely that the nozzle has another function besides the generation of the particles, namely the sufficient mixing of the liquid. This has two effects. First, the fluid mechanical state of the fluid changes into a two-phase liquid which becomes clearly visible, as the liquid becomes milky when the kinetic energy entering the liquid reservoir matches the liquid volume. This may promote the generation of smaller particle size distributions. Secondly, the background turbulence within the liquid reservoir increases strongly and causes an enhanced shearing of the bubbles which carry the particles to the liquid surface. This shearing process, caused by the turbulent mixing, can be seen as an active impactor which may enhance the rejection of large particles. These assumptions were supported by measurement of the volumetric particle size distribution of a 12-hole nozzle which was dipped into a large liquid reservoir whose fluid mechanical state and turbulence level could not be altered sufficiently by the kinetic energy entering from the jet. Further evidence and additional information on this subject can be found in [43].

\subsubsection{Flow visualisation}

In order to examine the generation, movement, and delivery of the particles above the liquid surface as a function of the pressure, a reference and Laskin nozzle, each with only one single $1 \mathrm{~mm}$ drill, were operated in DEHS (vegetable oil is less suited for optical flow analysis due to the strong absorption). For this visualisation the centreline of the air-jet was illuminated with a vertically arranged short pulsed laser light-sheet and the scattered light was recorded by means of a CCD camera. Figure 2.7 shows the global structure of the air jet from the reference nozzle (left) and Laskin nozzle (right) for various pressure conditions (top to bottom: $p=0.5,1,2,4$ bar). The increasing horizontal extension of the jets with increasing exit velocity and the decreasing divergence (top to bottom) are clearly visible. It can also be seen that the size of the bubble structures changes with increasing free-stream velocity due to the stronger mixing with the surrounding medium. The white circle in the left column reveals a delivered particle cloud which moves upwards in the form of a vortex structure with entrainment, and at the liquid surface of the lower right images, a bubble is visible just before the bursting takes place. The white circles and arrows in the right column indicate the rising bubbles emerging from the liquid feed holes of the Laskin nozzle, as mentioned in the previous section. This indicates that the holes in the ring are not operating in a way assumed in the literature. Figure 2.8 shows a selection of three images recorded independently whereby the white and and black squares in the left image indicate the size and location of the other two high resolution pictures. The jet axis (see bright centreline of small white square) is approximately $40 \mathrm{~mm}$ below the liquid level (see dark line within black square). The white circles in the centre image indicate particle filled air bubbles below the liquid level (see white dots) moving within the liquid, and the right image reveals a particle filled air bubble, which has penetrated the liquid surface, immediately before bursting. So it is evident that the particles are generated at the nozzle exit and transported within expanding air bubbles, which are exposed to the turbulent motion of the surrounding fluid on their way to the liquid surface, where the particles are delivered. 

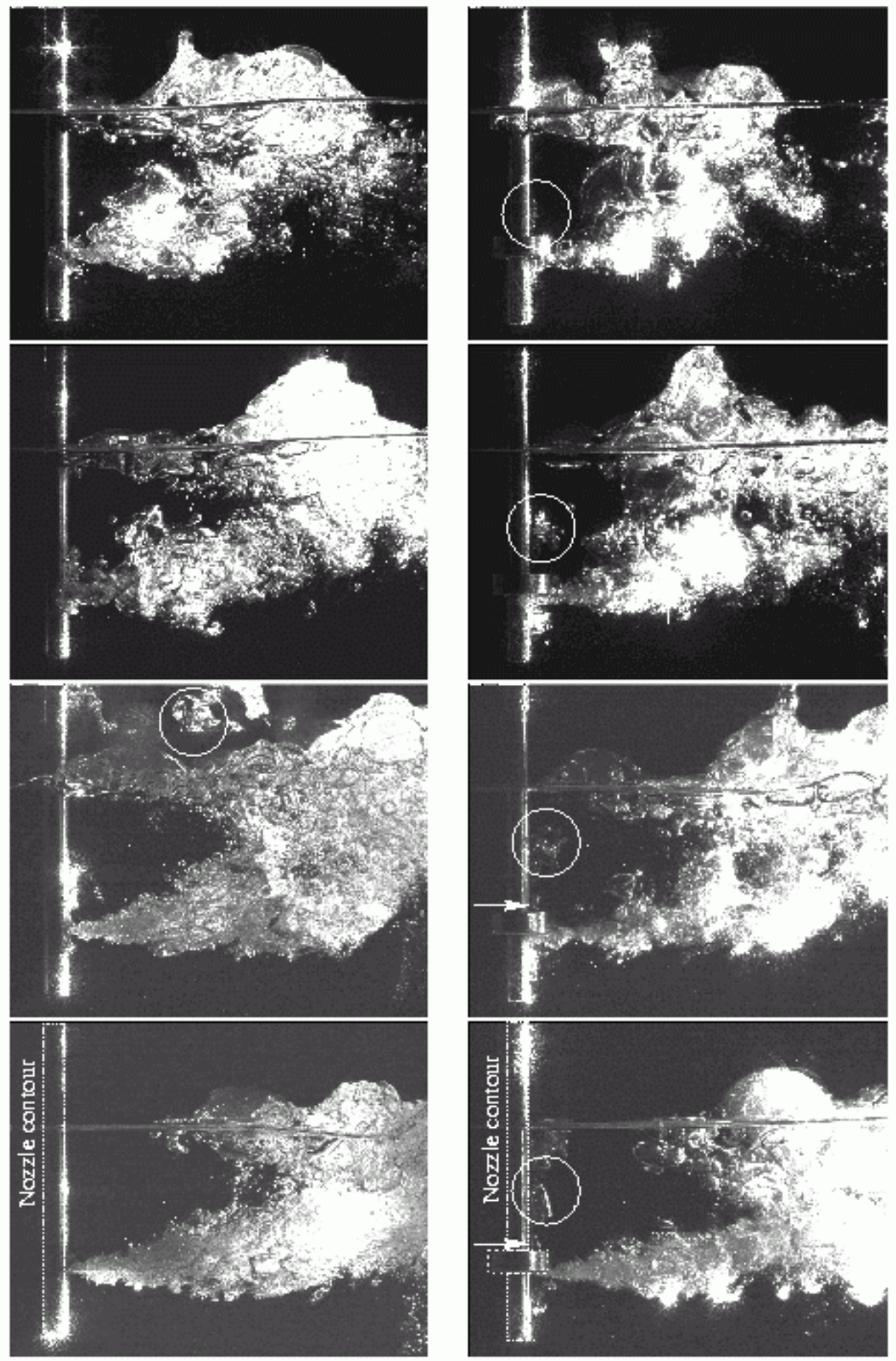

FIGURE 2.7: Laser light-sheet visualisation of an air jet emerging from a reference (left column) and Laskin nozzle (right column) in DEHS for various pressure conditions (top to bottom: $p=$ $0.5,1,2,4$ bar). The white circle in the left column reveals delivered particle cloud. Circles and arrows in the right column indicate rising bubbles emerging from the liquid feed holes of the Laskin nozzle. 

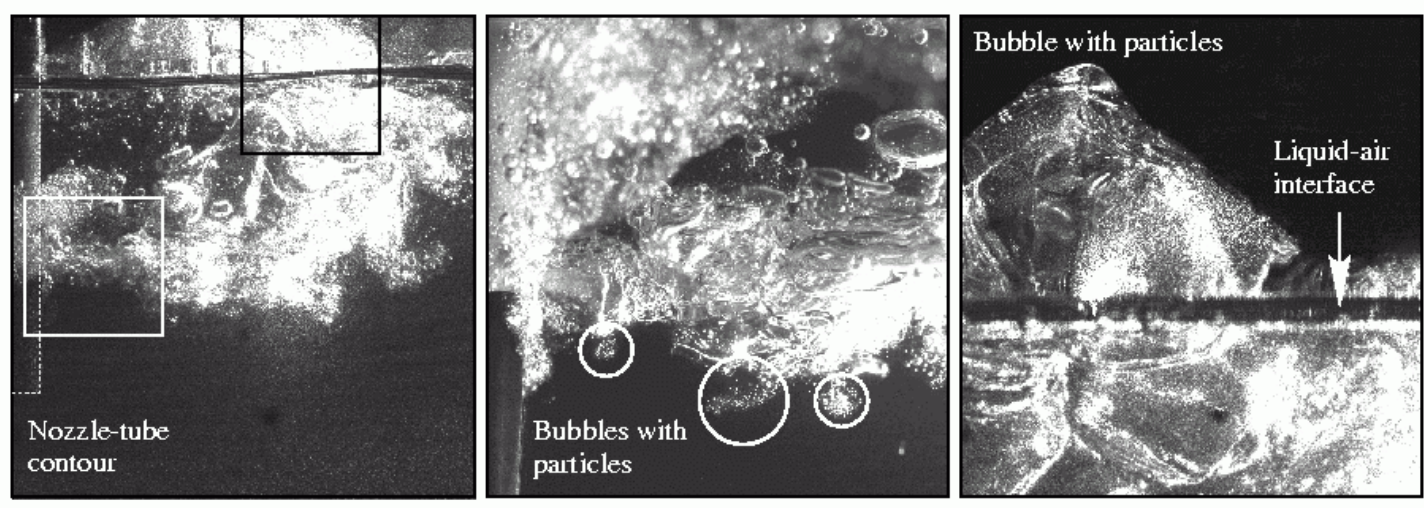

FIGURE 2.8: Left: Laser light-sheet visualisation of an air jet interacting with the liquid inside an aerosol generator for tracer-particle generation ( $p=1 \mathrm{bar})$. Centre: detail picture of particle filled air bubbles immediately behind the nozzle exit. Right: detail picture of particle filled air bubbles above the liquid surface.

\subsubsection{Conclusion}

It could be shown that high concentrations of particles with a narrow band size distribution and a mean diameter below $1 \mu \mathrm{m}$ can easily be generated by means of multi-hole nozzles under over-critical pressure conditions ( $\geq 1$ bar) provided the kinetic energy, entering the liquid volume, is sufficient to change the fluid mechanical state of the liquid to a highly turbulent two-phase fluid. This experimental result can be explained by assuming that the nozzle has basically three functions. Firstly, it generates the particles at the nozzle exit. Secondly, it changes the fluid mechanical state of the fluid into a two phase liquid which seems to promote the generation of smaller particle size distributions, as the fluid mechanical parameters are different. Thirdly, the remaining kinetic energy which is not consumed for the generation of the particles or the transition of the fluid mechanical state is transferred into the turbulent motion of the liquid and may act as an active impactor as the shearing of the bubbles which carry the particles to the liquid surface is enhanced. Besides this result, it could be qualitatively shown that bubbles arise from the liquid-fed holes of the Laskin nozzle. This implies that no particles can be generated at the ring side of the jet, as assumed in the literature, due to the missing liquid in this region.

All validation experiments, discussed in chapter 2 to 4, were performed with a multihole nozzle oil atomiser. For the experimental investigations described in chapter 5 to 7 the particles were generated with a smoke generator because of the large observation distance and the small aperture of the optical system. Because of the small free-stream velocity, the velocity lag of the particles is negligible relative to the uncertainties due to the recording and evaluation methods, which will be described in the following sections.

\subsection{Registration of the particle images}

Beside the particle dynamics, the registration, storage and read-out of the individual particle images is another key element in PIV because the accuracy of the technique strongly depends on the precision with which the image displacement can be related to particle locations 


\section{Particle Image Velocimetry}

and their respective particle displacements $[3,105]$. In the recording process the continuous intensity distribution of the particle images is transformed into a discrete signal of limited bandwidth as shown in figure 2.9 .
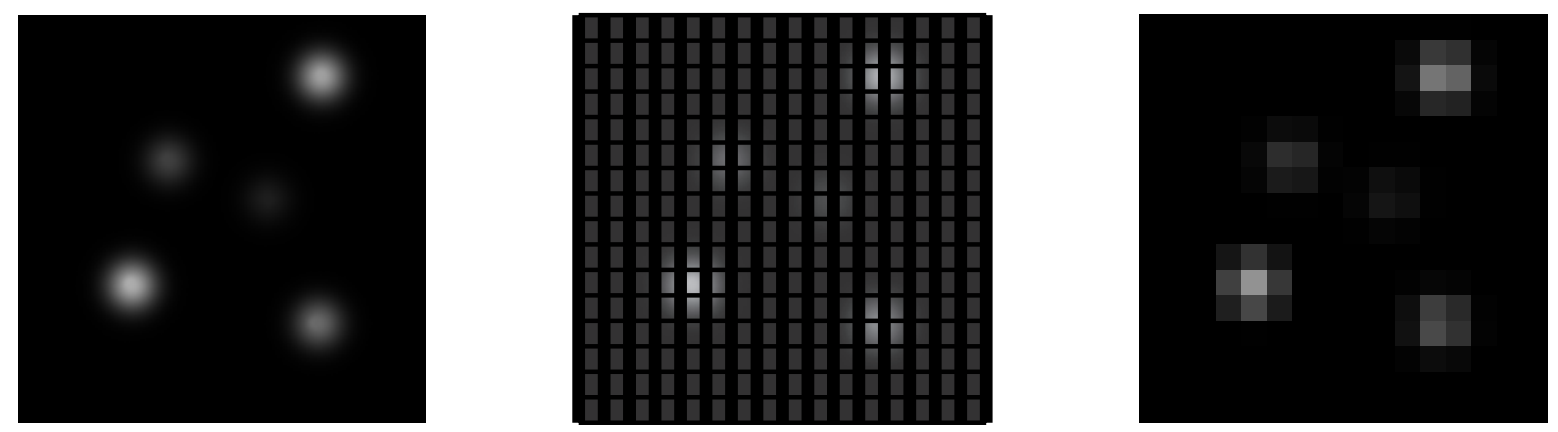

FIGURE 2.9: From left to right: Continuous intensity distribution of tracer-particles in the image-plane. Integration of the photons registered by the light sensitive area of a CCD sensor with fill factor smaller one. Discretised intensity distribution in memory of computer.

When the discretisation of the image-signal matches the minimum sampling rate, the frequency contents of the original signal can be reconstructed in principle without any losses and thus the particle location as well. This is the contents of the sampling theorem which states that a bandwidth-limited signal can be perfectly reconstructed from its discrete samples when the sampling rate of the signal is at least twice the signal bandwidth. Due to the noise introduced by inhomogeneous illumination or absorption by the surrounding fluid, optical aberration, the evaluation technique and the common peak-fit routines for sub-pixel accuracy, for example, the principal resolution given by the aperture, focal-length, wavelength of the monochromatic light and the magnification of the imaging system never need to be fully resolved in practice. This allows the use of digital recording systems which are easy to handle and the time consuming focusing, film change, developing and scanning procedure, for the photographic recording methods utilised some years ago, are no longer required [104, 109]. But it should be emphasised that beside the loss-of-information due to the discretisation of the spatial coordinate with a limited resolution as indicated in figure 2.9, and beside artefacts like Moire or Mach-band effects [35], a possible modification of the incoming intensity signal, due to the grey-level quantisation, nonlinearities in the pixel response or during the read-out of the images or AD-conversion, amplification and transportation via long connections, may reduce further the performance of the measurement technique. As a digital camera is a complex system this bias may be introduced in many different ways and its strength strongly depends on the CCD (charge coupled device) architecture and pixel (picture element) characteristics. To detect possible CCD errors in the recordings and to understand their consequences for PIV, a deeper understanding of the CCD principles is required.

\subsubsection{Principles of CCD sensors}

State-of-the-art CCD sensors are highly integrated semiconductor-circuits consisting of a huge number of adjacent MOS-diodes (metal-oxide semiconductor) each of which is composed of a polysilicon-oxide-layer (n-layer or anode), typically $0.5 \mu \mathrm{m}$ in thickness, and of the impoverishment layer. By applying a sufficiently high voltage between both layers the unbounded charges under the oxide-layer disappear and a light sensitive impoverishment-layer with a 
positive space-charge region occurs. In the impoverishment-layer the electron-hole pairs generated by penetrated photons of appropriate energy are separated according to their charge by the electric field. Electrons migrate under the pixel-register where they will be stored so that they can contribute to the signal. The holes, on the other hand, migrate in the opposite direction and vanish. Electron-hole pairs generated in the polysilicon- oxide-layer do not contribute to the signal because of their small drift velocity. The same holds for electron-hole pairs generated in the non-impoverishment-layer volume. As this region is field free, the charges perform a random movement until they recombine. For the read-out process the accumulated electrons have to be transferred sequentially from one MOS-element to the next up to the frame grabber by changing the potential of the MOS-diodes in an appropriate way. This requires that each pixel is usually composed of three adjacent diodes (triple-phase pixel-structure). As a consequence the fill-ratio, defined as the ratio of the light sensitive pixel-area to the total pixel-size, is strongly reduced and thus the sensitivity of the sensor as well. This can be partially compensated by using micro-lenses on top of each pixel but their transfer function may influence the signal as well.

\subsubsection{Quantum efficiency and signal-to-noise ratio}

A measure for the sensitivity and efficiency of each pixel is given by the quantum efficiency, defined as the number of generated electrons per photon of a given frequency. This value mainly depends on the spectral photon absorption line in silicon, on the thickness of the impoverishment-layer, on the thickness of the metal-oxide layer the photon has to pass before it may generate a electron-hole pair, as well as on the reflectivity of the metal-oxide layer, on the fill ratio of the pixel and finally on the diffusion-length of the electrons in the silicon which contribute to the signal.

For the imaging of weak bright objects, like the tiny particles inside the fluid, the quantum efficiency must be extremely large with respect to the total noise $N_{\mathrm{t}}$. Basically $N_{\mathrm{t}}$ is the sum of four independent noise sources, namely $N_{\mathrm{t}}=\left(N_{\mathrm{r}}^{2}+N_{\mathrm{d}}^{2}+N_{\mathrm{ph}}^{2}+N_{\text {cte }}^{2}\right)^{1 / 2} \cdot N_{\mathrm{r}}$ denotes the noise due to the readout electronic which depends on the design of the CCD amplifier, the main amplifier filtering, the read-out speed and the temperature. By increasing the read out speed the noise will increase too. This effect can be compensated by reducing the temperature of the CCD sensor. $N_{\mathrm{d}}$ is known as the dark current noise which is caused by the dark-charge $S_{\mathrm{d}}$, which again is caused by generation of electron-hole pairs at defects in the semiconductor, which accumulates during the illumination time inside the pixel. $N_{d}$ and $S_{d}$ are related according to $N_{\mathrm{d}}=\sqrt{S_{d}}$. $S_{\mathrm{d}}$ is directly proportional to the integration time and decreases exponentially with the temperature. Generally $S_{\mathrm{d}}$ drops by a factor of two by decreasing the temperature by $7^{\circ} . S_{\mathrm{d}}$ can be reduced further by 2 to 3 orders of magnitude using the multi-phase binned-mode which is quite common for a new generation of CCD sensors. The photon-noise $N_{\text {ph }}$ is caused by the statistical probability of convective photo-flux in the sensitive region. This quantity is proportional to the square-root of the photon-flux $N_{\mathrm{ph}}=S_{\mathrm{ph}}^{1 / 2}$ and can be neglected for small photon-flux. $N_{\text {cte }}$ results from the incomplete charge transfer from the generation point to the read-out node. Scientific-CCDs possess a transfer efficiency of 0.9999 and more which reduces the charge after 1000 shifts by $10 \%$. Although this reduction can be accounted for via calibration the statistical variation of the charge-leak results in a signal uncertainty.

For very intense objects the described noise is of minor importance compared to the so 


\section{Particle Image Velocimetry}

called storage-capacity because, when the storage capacity is reached, a flux of charges into the neighbouring pixel will occur and alter the stored information in all pixels involved. In PIV this blooming effect can be observed, for example, by recording sufficiently strong reflections from surfaces. The pixel storage capacity of commercially available CCD sensors is typically $10^{5}$ to $10^{6}$ electrons mainly depending on the pixel-size, on the manufacture technology and on the registration-voltage. Thus, the dynamic range of the CCD electronics can reach in principle 16 bit and more.

\subsubsection{CCD architecture}

To visualise quantitatively the influence of the fill-ratio (the fraction of the light sensitive area of the total pixel size) and the pixel response function of the CCD sensor, the convolution of the geometrical particle image with the point spread function of the lens system has to be convoluted with the two-dimensional point spread function of the pixel. Whereas the first function can be derived analytically for an aberration free lens with a limited spatial bandwidth, the complexity of the real pixel response requires an appropriate model. To simplify matters, the ideal intensity distribution in the image plane will be weighed with a linear pixel sensitivity function with a positive slope and values between zero and one in the domain $n \leq x \leq n+1$ for all $n \in \mathbf{Z}$ according to equation (2.3).

$$
I(x)=\frac{1}{\sigma \sqrt{2 \pi}} \exp \left[-\frac{\left(x-x_{0}\right)^{2}}{2 \sigma^{2}}\right] \cdot\left(x-x_{0}-n\right)
$$

Although a simple first order approximation is quite a strong idealisation, it is well suited to model the basic noise terms discussed in the previous section, like non constant impoverishmentor metal-oxide layer, systematic variations in the reflectivity or poorly aligned or manufactured micro-lenses, for example. The continuous graph in figure 2.10 represents the Gaussian intensity distribution of the diffraction limited image of a point source in Fraunhofer approximation as a function of the spatial coordinate (first factor in equation (2.3)). The solid lines show the local intensity distribution weighed with a linearly varying pixel response function according to equation (2.3). The dashed lines indicate the accumulated light for each individual pixel calculated from the unbiased signal and the long-dashed dotted graphs show the same function but calculated from the linearly weighed signal (dotted graphs). As the dashed line is always above the dashed dotted line, it is obvious that the linear response of the pixel lowers the signal strength to a large extent and thus the contrast in the recording. As a consequence, the signal-to-noise ratio decreases and the size of the particle image may be reduced when the light accumulated at the outer parts of the distribution is below the threshold of digital registration. These effects can be partially compensated by increasing the light-sheet intensity, by changing the aperture of the imaging system or by increasing the particle size, for example, but the modification of the particle image shape caused by the pixel response results in an erroneous velocity estimation, as can be easily seen by calculating the centre of gravity over all intensities. When the upper left graphs are considered, the equal intensity values in case of the unbiased signal lead to the conclusion that the maximum of the continuous intensity distribution is exactly symmetric with respect to the interface between two adjacent pixels. The weighed distribution, on the other hand, suggests that the maximum is slightly displaced to the real location as the difference between the dashed and the dashed dotted graph increases in positive pixel direction due to the second term in equation (2.3). If the maximum of the 

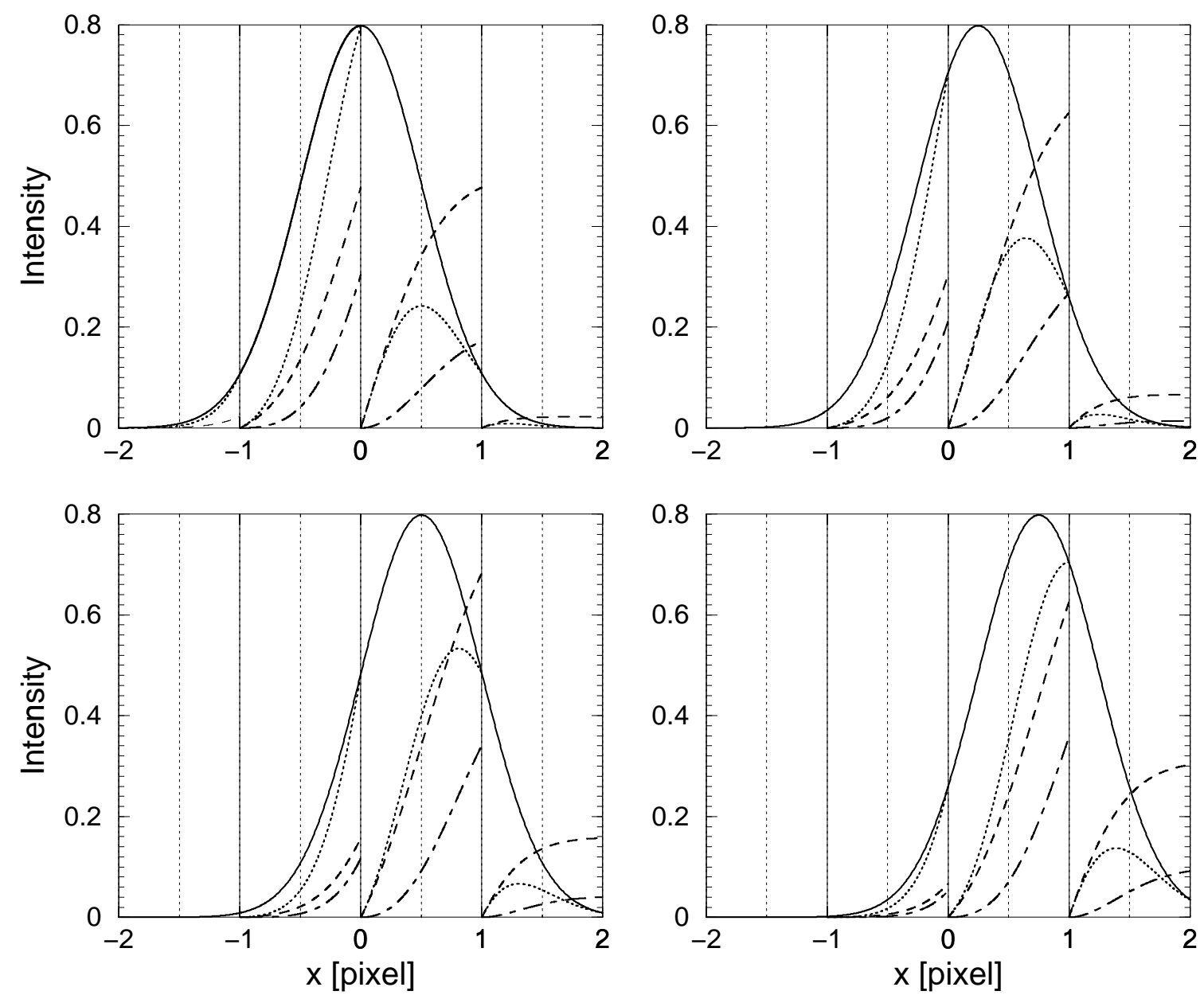

FIGURE 2.10: Gaussian intensity distribution of a particle image (solid line) and its discrete representation as a function of the fill-factor, pixel response and sampling location (upper left to lower right). The dashed lines within the Gaussian distribution indicate the collected intensity calculated for the unbiased signal as a function of the linear pixel dimension and the long-dashed dotted line the modified intensity value (integration over the dotted graphs).

Gaussian intensity distribution is not symmetric relative to the interface between two adjacent pixels, as shown in the two lower and the upper right graphs of figure 2.10, the unbiased signal increases continously from 0.48 to 0.68 , in the example, until the centre of the pixel coincides with the maximum of the Gaussian curve (lower left graphs). Under these conditions the greyvalues of the measured intensity is again completely symmetric with respect to the continuous distribution so that the exact location of the original function is identical with the discrete sample. To examine the behaviour of a small fill-ratio sensor, the intensity of the dashed and dashed dotted graphs can be compared at a particular sub-pixel location. A comparison indicates the minor importance of this parameter as long as the particle image size covers on average three pixels in each spatial direction. Under these circumstances the measurement precision may be reduced as the differences between neighbouring pixel-intensities decrease while the influence of any superimposed noise increases, but this can be compensated by using the appropriate components for the experiment. If the particle image size is in the range of 


\section{Particle Image Velocimetry}

a pixel, either due to the low sensitivity of the sensor or to the noise, the fill ratio becomes important as the complete image information can disappear when the intensity distribution is located between two adjacent light sensitive areas. Due to the statistical evaluation, which will be described in the following section, the displacement can still be calculated as the probability that all particle images within an interrogation area are unpaired is small, but an increase in noise is unavoidable due to unpaired particle images and to the poor performance of the centre of gravity approach or related methods, see section 2.4 and 2.4.1. This is different when the displacement estimation relies on a single image pair as in particle tracking analysis or as forced in [27]. However, under these conditions the measurement accuracy is strongly affected by the CCD characteristics.

\subsection{Particle image analysis}

In order to extract the displacement information from the two single exposed grey-level patterns acquired at $t$ and $t^{\prime}$, both images are usually evaluated by means of statistical evaluation techniques, for two reasons. First, individual particle image pairs cannot be identified sufficiently reliable due to the high seeding concentration. Secondly, the statistical evaluation is less sensitive to noise and discretisation effects as outlined in the previous section. For the evaluation, the whole image is sampled with an appropriate step-size, typically half the linear dimension of the grey-level sample $I(x, y)$. For each sampling location a two-dimensional grey-level sample $I(x, y)$ of certain size and shape is extracted from the source image as indicated in figure 2.1, and it is cross-correlated with the corresponding sample $I^{\prime}(x, y)$ from the search recording according to the following equation ${ }^{1}[52,105,109]$.

$$
R_{I I^{\prime}}(x, y)=\sum_{i=-n}^{n} \sum_{j=-m}^{m} I(i, j) I^{\prime}(i+x, j+y)
$$

The discrete cross-correlation formula produces one cross-correlation value $R_{I I^{\prime}}(x, y)$ for each possible displacement between the two samples by calculating the sum of the products of all overlapping pixel intensities so that the output of this calculation can be displayed in the form of a two dimensional cross-correlation plane, see figure 2.11. Due to the symmetry of the cross-correlation function the template $I(x, y)$ can be selected in either of the two images, but the sign of the displacement has to be reversed when the template originates from the image recorded at $t+\Delta t$. The cross-correlation formula is usually properly normalised so that its amplitude is invariant under scale changes in the amplitude of $I$ and $I^{\prime}$ and thus not affected by variations in the particle image number, size and intensity. This allows to quantify the quality of the signal which is of great importance when aligning the experimental components, such as light-sheet and cameras, with respect to each other, see figure 2.12 .

\footnotetext{
${ }^{1}$ As each single grey-level sample back-projected in the object space and multiplied by the effective light-sheet thickness, given by the intensity distribution of the light-sheet itself, the particle size distribution, the imaging optics and sensitivity of the CCD camera, represent a fluid volume, the cross-correlation can be considered as weighed volume average.
} 


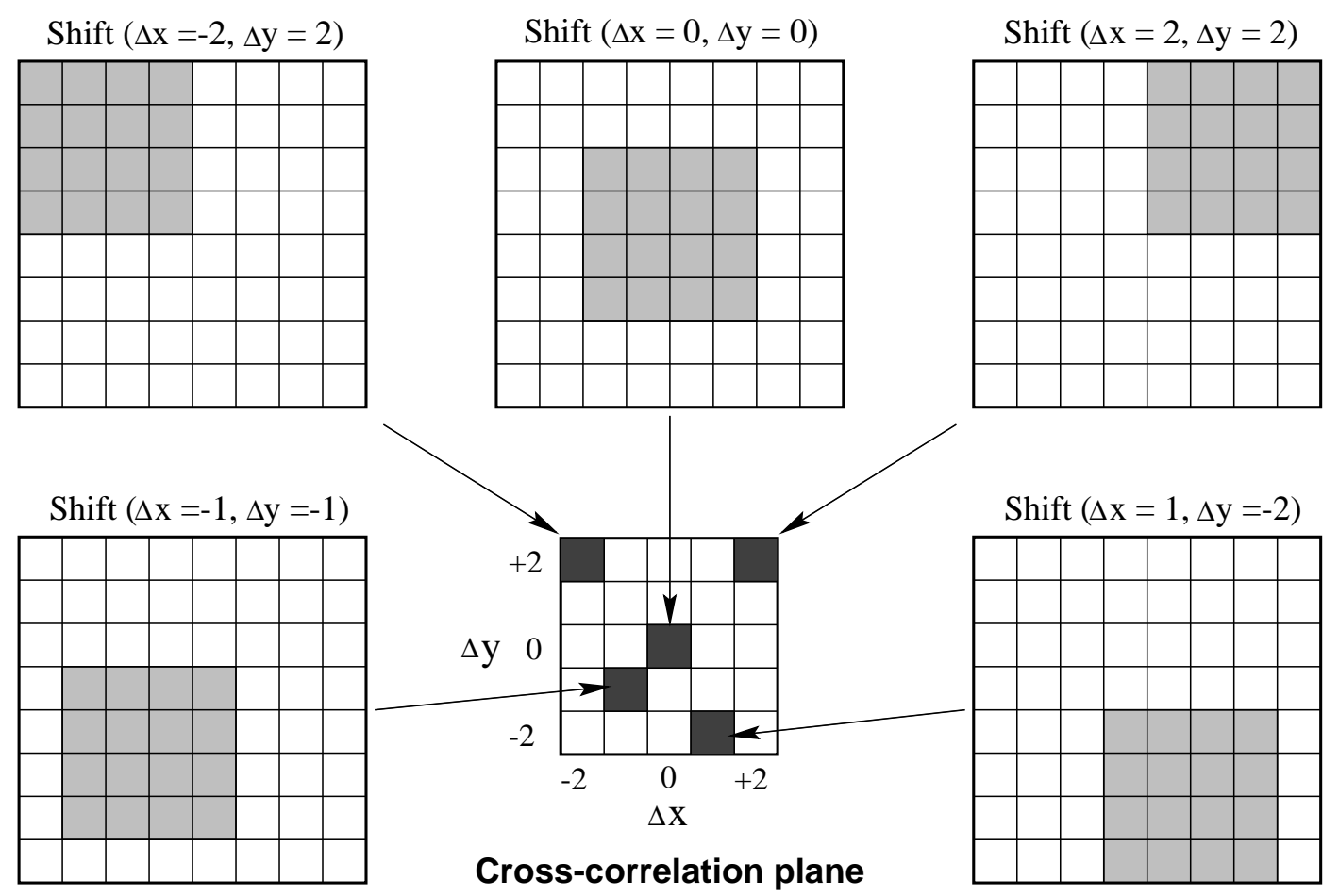

FIGURE 2.11: Example of the formation of the correlation plane by direct cross-correlation: here a $4 \times 4$ pixel template (grey-square) is correlated with a $8 \times 8$ pixel size sample to produce a $5 \times 5$ pixel cross-correlation plane.

$$
c_{I I^{\prime}}(x, y)=\frac{\sum_{i=-n}^{n} \sum_{j=-m}^{m}[I(i, j)-\bar{I}]\left[I^{\prime}(i+x, j+y)-\overline{I^{\prime}}\right]}{\sqrt{\sum_{i=-n}^{n} \sum_{j=-m}^{m}[I(i, j)-\bar{I}]^{2}} \sqrt{\left.\sum_{i=-n}^{n} \sum_{j=-m}^{m}\left[I^{\prime}(i+x, j+y)-\overline{I^{\prime}}\right)\right]^{2}}}
$$

$\overline{I^{\prime}}$ denotes the mean intensity value of the search window calculated over the portion which coincides perfectly with the $I(i, j)$ and $\bar{I}$ is the corresponding mean intensity for the template. It is obvious that $\overline{I^{\prime}}$ has to be calculated for each possible shift to guarantee that $0 \leq c_{I I} \leq 1$ (negative values are usually not considered as intensities are always positive and the mean value is close to zero for moderate image densities). As a direct implementation of this formula in the spatial domain becomes quite time intense, especially for large correlation windows, the computation of the correlation is usually performed in the frequency domain via fast Fourier transformation (FFT) based algorithms. They are fast as symmetry properties are taken into account but artifacts like aliasing, circular effects and limitations in the size and shape of the correlation or interrogation window may lower the quality of the velocity estimation and restrict the performance of the method for particular applications, see chapter 3 . As the cross-correlation approach is based on the idea that the location of the signal-peak yields the information about the displacement of the image pattern, the conditions for the correctness of this assumption need to be analysed in detail. Due to the fact that this is mathematically not tractable when a single realization of a particle image pattern is considered, the pattern is thought of as a single realization of a random process and it is assumed that this particu- 


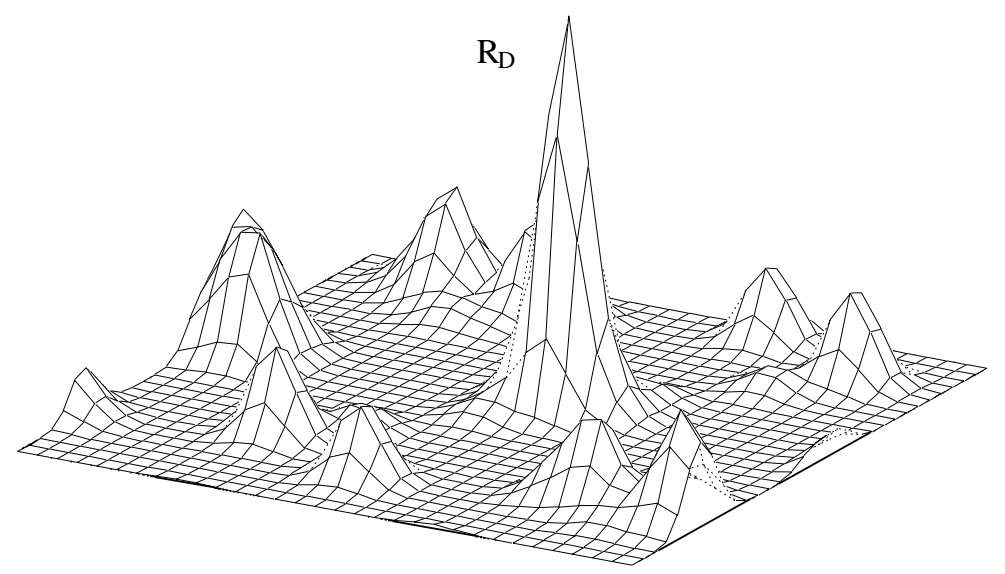

FIGURE 2.12: Spatial distribution of the the cross-correlation coefficient calculated over five randomly located particle image pairs of varying intensity moving homogeneously $\Delta x=1.89$ pixel, $\Delta y=$ 1.89 pixel. The displacement correlation peak $R_{D}$ is surrounded by random noise due to other pairing possibilities. Whereas $R_{D}$ is proportional to the number of paired particle images as only these add to the signal strength, the height of the noise peaks depends on the intensity of the images and on the organisation of the underlying pattern.

lar pattern represents the entire random process to which it belongs ${ }^{2}$. Under this assumption, it can be shown by analysing all possible tracer patterns for a single flow field realization that the particle images must be exactly identical in size, shape and intensity, homogeneously distributed and the structure of the pattern must be invariant under spatial transformations to ensure that the particle image displacement is directly related to the location of the highest correlation peak as assumed in PIV evaluation [2, 52, 104]. In practice, of course, the required conditions are only approximately realized, so that experimental, numerical and analytical investigations are required to analyse the effects of non ideal conditions introduced by the following: inhomogeneities in the particle image distribution, variations in the particle image sizes, shape and intensity, image sampling, grey-level quantisation as well as non-linearities in the pixel response or light sensitivity, shot noise, charge leakage, dark-current noise in the CCD and electronic noise in the CCD, camera and frame grabber, gradients and so on.

\subsubsection{Particle image density, loss of pairs and velocity gradients}

In the previous sections it has always been assumed that the signal peak $R_{D}(x, y)$ is uniquely defined, reliable to detect and not affected by random noise superimposed on the signal peak. In order to obtain this ideal conditions in any real experiment, the following relation has to be taken into account while setting up the experiment $[2,52,104]$.

$$
R_{D}(x, y) \sim N_{I} F_{I} F_{O} F_{\Delta}
$$

$N_{I}$ represents the so called image density which is actually the number of particle image pairs in the interrogation domain which contribute to the signal strength. $F_{I}$ and $F_{O}$ denote the in-plane and out-of-plane loss-of-pairs as a result of the finite size of the measurement volume and $F_{\Delta}$ accounts for the loss of correlation due to gradients within the measurement volume.

\footnotetext{
${ }^{2}$ For ergodic processes it is possible to derive the desired statistical information about the entire random process from an appropriate analysis of a single arbitrary sample function.
} 


$$
\begin{aligned}
N_{I} & =\frac{C \Delta z_{O}}{M^{2}} D_{x} D_{y} \quad N_{I} \in \mathbf{N} \\
F_{I} & =\left(1-\frac{|\Delta x|}{D_{x}}\right)\left(1-\frac{|\Delta y|}{D_{y}}\right) \\
F_{O} & =\left(1-\frac{|\Delta z|}{\Delta z_{0}}\right) \\
F_{\Delta} & =\exp \left(-\frac{M|\Delta u| \Delta t}{d_{\tau}}\right)
\end{aligned}
$$

Equation (2.6) states that the amplitude of the cross-correlation peak increases with increasing image density $N_{I} \in \mathbf{N}$ and decreases with increasing displacement, no matter in which direction or if the displacement of the particle images is not constant. In order to optimise the performance of the experiment, the number of paired particle images is of primary importance as only these add to the signal strength. In addition the number of correct pairings with respect to incorrect combinations has to be optimised for increasing the signal-to-noise ratio and thus the detectability of the signal. This can be achieved either by changing the seeding concentration, the magnification of the imaging system or simply by increasing the light-sheet thickness, see equation (2.7). In-plane loss-of-pairs, caused by particles entering or leaving the measurement volume during the illuminations, due to the motion of the fluid, can be reduced by decreasing $\Delta t$ or eliminated when the search window contains the corresponding particle image pattern selected with the template. This can be achieved either by correlating differently sized samples as described before or by using window-shifting in connection with multi-pass interrogation techniques [106, 109]. Using this approach two equally sized samples, separated by the local particle image shift are cross-correlated after the local shift has been determined within a first evaluation. Since the correlation windows are symmetrically shifted with respect to each other and relative to the exact measurement position, it becomes necessary to determine where the footprint of the vector should be located (centre or corner). This is especially important when different evaluation methods are compared because the particle pattern employed for the evaluation may be different for the same pixel coordinates of the displacement vector [97]. Out-of-plane loss-of-pairs can be compensated by shifting the light-sheet in the direction of the mean particle displacement, as proposed in [52], or by using multi-light-sheet arrangements when the out-of-plane motion is not uniform, see chapter 4 . The effect of in-plane gradients can be reduced by increasing the magnification of the imaging system, by decreasing the time-separation between the two illuminations or by using properly shaped interrogation windows whose linear dimension is reduced in the direction of the gradient.

\subsubsection{Signal-peak detection and displacement determination}

In order to increase the accuracy in determining the location of the displacement peak from \pm 0.5 pixel to sub-pixel accuracy, an analytical function is fitted to the highest correlation peak by using the adjacent correlation values [109]. Usually two one-dimensional Gaussian fits along the two coordinates through the highest correlation coefficient are applied. The motivation for this particular function is based on the fact that under ideal imaging conditions the 


\section{Particle Image Velocimetry}

shape of the signal peak is of Gaussian shape, like a diffraction limited image of a particle, and close to 3 pixels in each spatial direction for realistic applications of PIV.

$$
f(x)=C_{0} \exp \left[-\frac{\left(x_{0}-x\right)^{2}}{k}\right]
$$

$x_{0}$ indicates the exact location of the maximum and $C_{0}, k$ are coefficients of no direct interest. Using this expression for the main and the adjacent correlation values and the fact that the first derivative of this expression must be zero, the position can be estimated with sub-pixel accuracy.

$$
\begin{aligned}
\Delta x_{i} & =i+\frac{\ln R_{(i-1, j)}-\ln R_{(i+1, j)}}{2 \ln R_{(i-1, j)}-4 \ln R_{(i, j)}+2 \ln R_{(i+1, j)}} \\
\Delta y_{i} & =j+\frac{\ln R_{(i, j-1)}-\ln R_{(i, j+1)}}{2 \ln R_{(i, j-1)}-4 \ln R_{(i, j)}+2 \ln R_{(i, j+1)}}
\end{aligned}
$$

Unfortunately, differences from this ideal Gaussian shape are normal due to the discretisation, electronic noise, velocity gradients and optical aberrations and introduce artefacts which reduce the performance of this peak-fit and lead to systematic measurement errors. The peaklocking effect for example which is introduced by under-sampling the particle images may be amplified by using this peak-fit due to the characteristic variation of the RMS error as a function of the sub-pixel location of the correlation peak.

Better results can be achieved by fitting a two dimensional Gaussian function to a larger number of points by using the iterative Levenberg-Marquardt method as described in [88]. The weighting of the values should be according to the Fisher transform in order to compensate the non-normal distribution of the correlation-coefficient error (the error is zero for values equal 1 and increases with decreasing amplitude). This peak-finder also works properly for nonGaussian shaped correlation peaks and is less sensitive to sub-pixel displacements compared with the three point Gaussian peak-fit.

Figure 2.13 shows the distribution of the measured $x$-displacements as a function of the sub-pixel shifts along the other coordinate calculated from simulated images with a size of $2048 \times 16024$ pixel $^{2}$. The evaluation has been performed by using the 2 nd order accurate FFT-based multi-pass interrogation procedure with three-point Gaussian peak-fit and the FFTbased free-shape cross-correlation along with the two dimensional Gaussian peak-fit. As the distribution of the measured velocity is nearly independent of the sub-pixel location when the two-dimensional Gaussian peak is applied, only the graph with the largest deviation from the true displacement has been plotted for comparison. The enormous variation of the measured displacement as a function of the sub-pixel shift in $y$-direction should be noted when the threepoint Gaussian peak-fit is applied, see figure 2.13 left. It results in a strong peak-locking effect compared with the two-dimensional Gaussian peak fit analysis despite of the large particle image diameter (2.4 pixel).

The mean and standard deviation following from the graphs in figure 2.13 are summarised in table 2.1. It should be noted that the reliability of the three-point peak-fit is strongly limited, with respect to the two dimensional Gaussian peak-fit, due to the variation of the standard deviation as a function of the sub-pixel location (one order of magnitude). For this reason, the analysis of the recordings acquired for the investigation presented in chapter 5 to 7 , was performed with the the iterative Levenberg-Marquardt method and the displacement estimation with sub-pixel accuracy was performed with a two dimensional Gaussian peak-fit routine. 

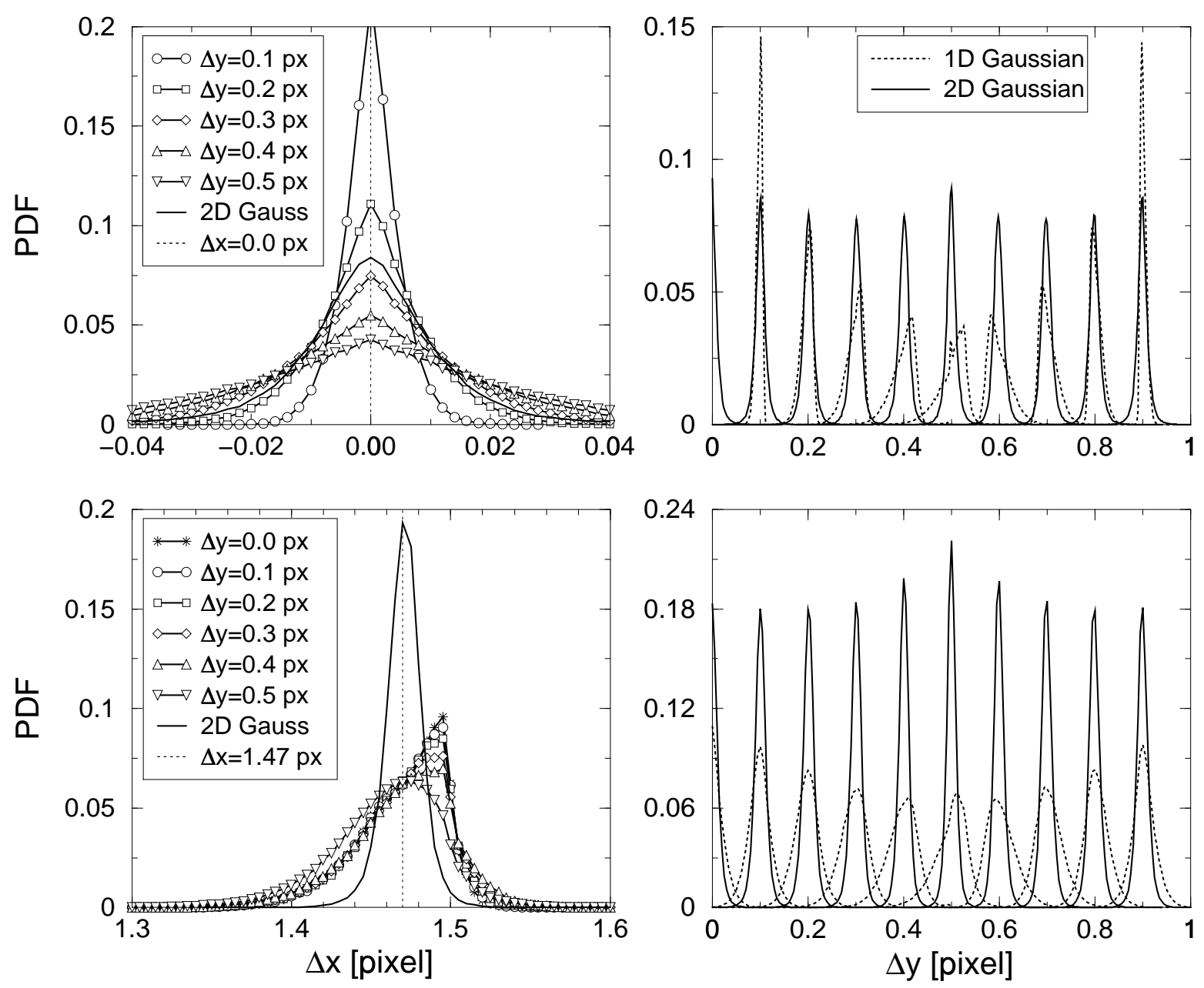

FIGURE 2.13: Numerical comparison between two-dimensional (solid lines) and three-point Gaussian peak-fit (graphs with symbols (left column) and dotted lines) with iterative Levenberg-Marquardt method for two fixed particle image displacements (top: $\Delta x=0$ pixel, bottom: $\Delta x=1.47$ pixel) as a function of the $y$-shift. The graph represents the probability density functions for a set of simulated displacement fields (each 2000 by 16000 pixel in size) analysed with a 2nd order accurate multi-pass interrogation technique with $32 \times 32$ pixel interrogation window and $50 \%$ overlap.

Figure 2.14 shows the distribution obtained by analysing experimental data. The displacement was achieved by transforming one image as will be outlined in the following chapter. It can

\begin{tabular}{ccc}
\hline$\Delta y$ [pixel] & $\Delta x$ [pixel] & $\Delta x$ [pixel] \\
\hline 0.0 & & $1.465 \pm 0.198$ \\
0.1 & $-0.003 \pm 0.020$ & $1.425 \pm 0.100$ \\
0.2 & $+0.003 \pm 0.038$ & $1.453 \pm 0.113$ \\
0.3 & $+0.013 \pm 0.061$ & $1.440 \pm 0.102$ \\
0.4 & $+0.005 \pm 0.071$ & $1.468 \pm 0.121$ \\
0.5 & $-0.005 \pm 0.085$ & $1.450 \pm 0.128$ \\
\hline 0.1 & $+0.015 \pm 0.085$ & $1.445 \pm 0.079$ \\
\hline
\end{tabular}

TABLE 2.1: Comparison between two-dimensional (bottom row) and threepoint Gaussian peak-fit for two particle image displacements in $x$-direction (centre column $\Delta x=$ 0 pixel, right column $\Delta x=$ 1.47 pixel) as a function of the $y$-shift. 


\section{Particle Image Velocimetry}

be seen that the functional dependence of the distributions on the displacement agrees nicely with the result shown in figure 2.13.

For completeness it should be mentioned that various techniques have been proposed in the past to optimise the evaluation procedure with regard to accuracy and spatial resolution and to overcome the limitations associated with the required image density and the tolerable gradients, for details the interested reader may consult the existing literature $[22,27,33,72$, 97]. The excellent performance of the 3-point Gaussian fit when the location of the correlation maximum is exactly symmetrical relating to a pixel stimulated Lecordier et al [72] to make use of the accuracy by transforming the measured image in such a way that the measured velocity becomes zero after the transformation, see section 3.2.2. To increase the spatial resolution without increasing the probability that a random correlation peak will exceed the height of the displacement peak and deteriorate the velocity measurement, it is possible to calculate the correlation twice with a small spatial separation in such a way that the image displacement
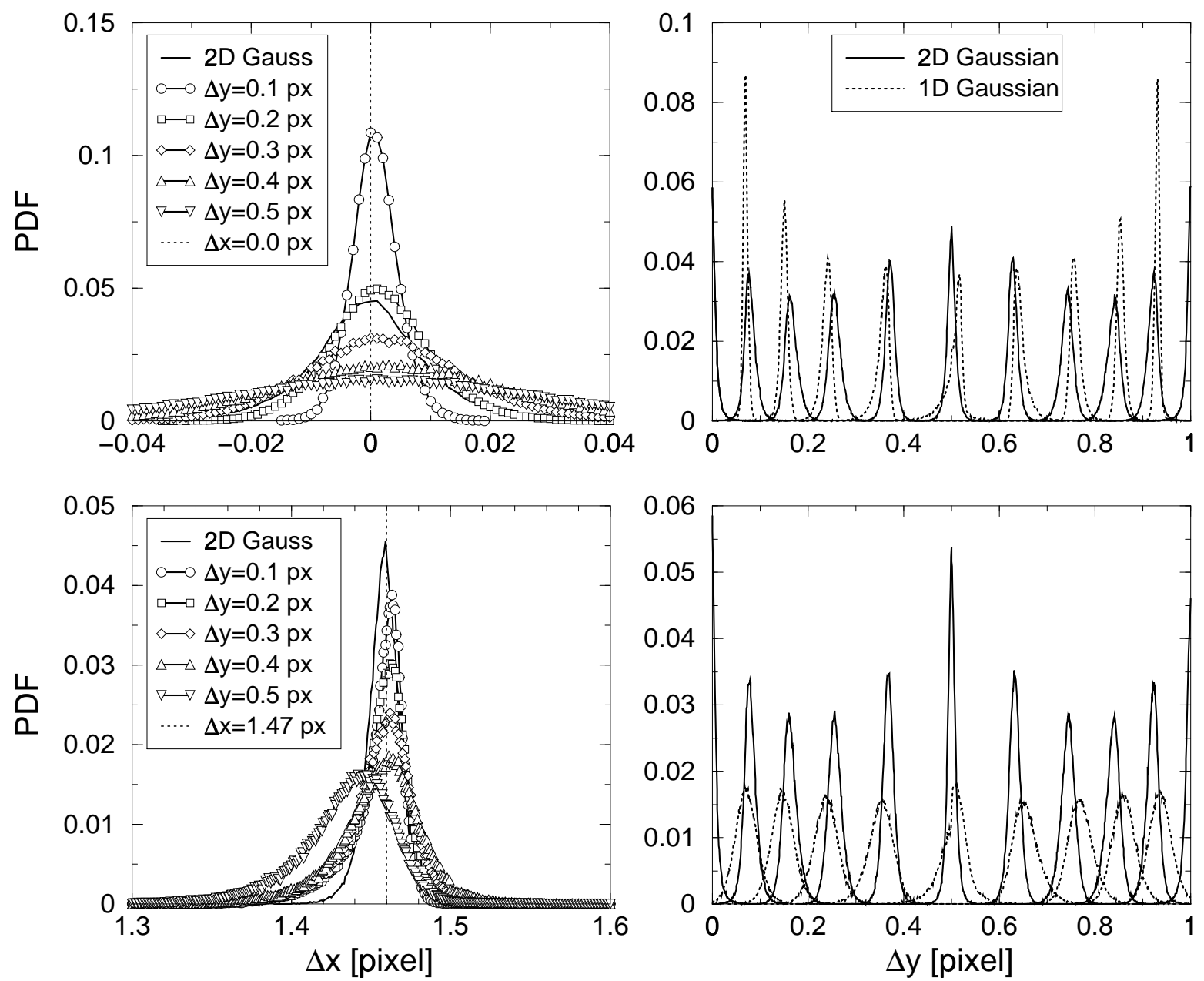

FIGURE 2.14: Experimental comparison between two-dimensional (solid lines) and three-point Gaussian peak-fit (graphs with symbols (left column) and dotted lines) with iterative Levenberg-Marquardt method for two fixed particle image displacements (top: $\Delta x=0$ pixel, bottom: $\Delta x=1.47$ pixel) as a function of the $y$-shift. The graph represents the probability density functions for a set of measured displacement fields (each 1280 by 1024 pixel in size) analysed with a 2nd order accurate multi-pass interrogation technique with $32 \times 32$ pixel interrogation window and $50 \%$ overlap. 
is identical but the noise uncorrelated [27]. Once this has been done, the signal peak can be clearly detected by calculating a correlation of the correlation (the uncorrelated noise will vanish), but again it should be taken into account that evaluation schemes based on few particle images are highly sensitive to noise that is introduced by the characteristics of digital cameras. In order to enhance the signal quality when strong in-plane gradients are present, Huang et al [33] has proposed to transform the image in such a way that the deformation vanishes after the mapping, see also [22]. This approach works fine as long as the flow under consideration possesses only in-plane gradients $(\partial u / \partial x, \partial u / \partial y$ and $\partial v / \partial x, \partial v / \partial y)$. In case of strong outof-plane gradients $(\partial u / \partial z)$ this and most of the other sophisticated methods will fail, as can be easily realized by considering a turbulent boundary layer experiment with a light-sheet parallel to the flat plate. Under these conditions the apparent particle image gradients caused by particles from different layers cannot be compensated because of arising instabilities in the image analysis. 
2 Particle Image Velocimetry 


\section{Stereo-scopic Particle Image Velocimetry}

The conventional PIV technique described in the previous sections yields reliable results as long as the flow under investigation is two-dimensional and parallel to the light-sheet. In case of turbulent flows with a strong velocity component being normal to the light-sheet, the outof-plane velocity component remains unknown and the in-plane components are biased due to the perspective error as indicated in figure 3.1 for a simulated flow with a pure out-of-plane velocity component.

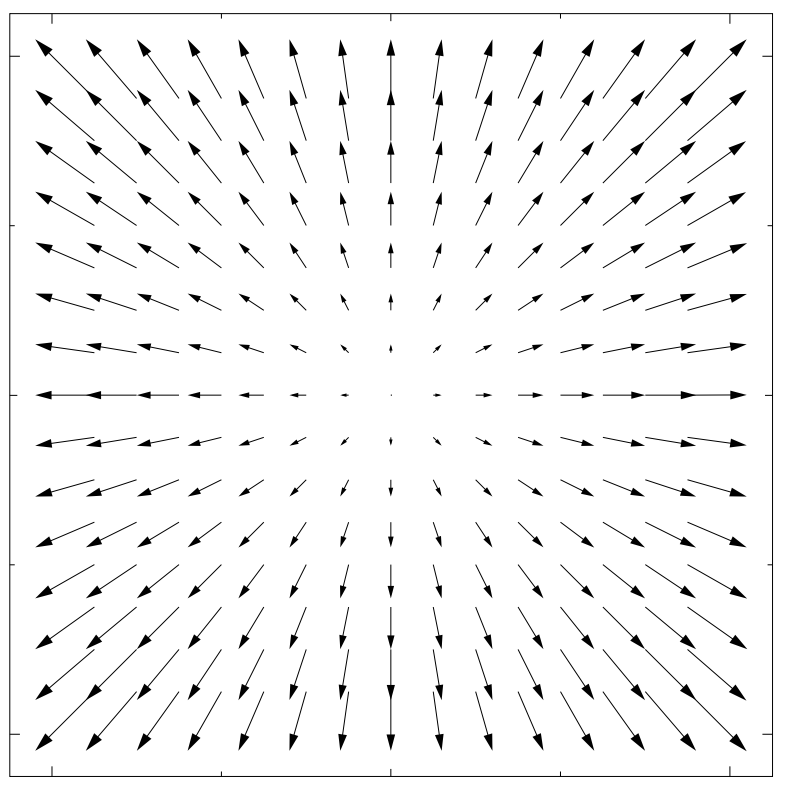

FIGURE 3.1: Magnitude and direction of the projection-error as a function of the image location for a constant out-of-plane displacement.

As this error is directly proportional to the viewing angle, according to figure 3.1 , the object distance must be increased to minimise the error while keeping the field of view. As this approach requires long focal length lenses it is obvious that this approach is not satisfactory. To overcome this constraint completely and to obtain the out-of-plane velocity component, a stereoscopic observation arrangement has to be applied, which will be outlined in the following sections.

\subsection{Principles}

Using the stereoscopic recording technique, the images of tracer particles are recorded simultaneously from two different viewing directions, and the correct displacement (without 
perspective error) of the particle ensembles are reconstructed by using the proper equations. The basic recording arrangements can be classified either with respect to the camera position relative to the light-sheet or with respect to the propagation direction of the light-sheet plane according to figure 3.2. The left drawing reveals a configuration where both cameras are located on the same side of the light-sheet. As a consequence, this recording arrangement can be operated in forward/backward or ninety degree configuration when the propagation direction of the light-sheet plane is considered. An alternative arrangement is shown in the right drawing of the same figure. In this case the cameras are separated by the light-sheet plane so that the pure forward, backward and ninety degree arrangements are possible. As long as only the intensity of the scattered light is considered, the most efficient light-sheet camera-configuration is the purely forward scattering set-up, according to the Miescattering diagram in figure 2.2, followed by the forward/backward configuration, purely backward and finally ninety degree case. This may change when the state of polarisation has to be taken into account beside the intensity (this will be further analysed in chapter 4 and the experimental parts of this thesis).
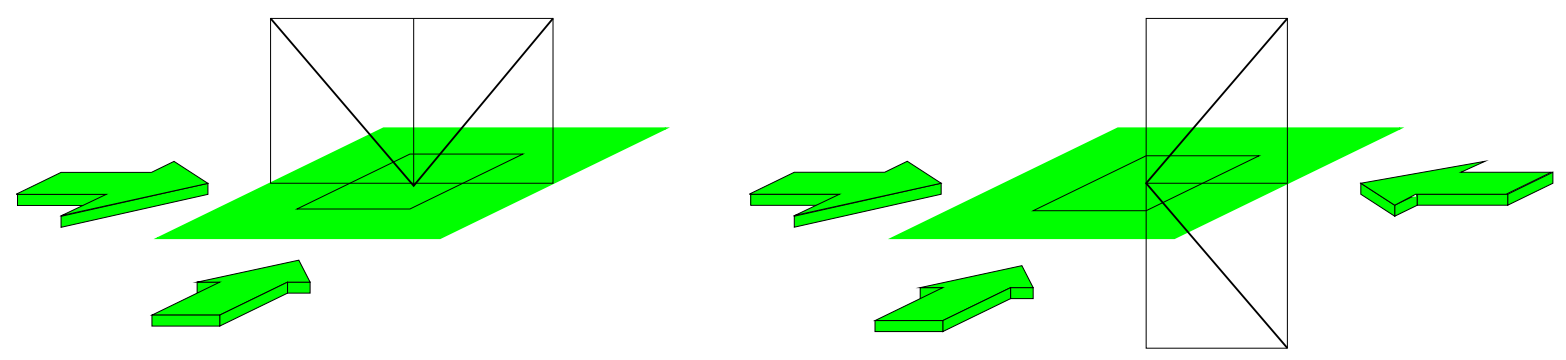

FIGURE 3.2: Stereoscopic recording arrangements. The orientation of the principle observation ray (oblique lines) relative to the light sheet (dark plane) or the propagation direction of the light-sheet plane (indicated by the arrows) can be used to define various recording configurations with different properties. The observation direction from one light-sheet side (left drawing) allows forward/backward and ninety degree imaging and from opposite sides (right drawing) forward, backward and ninety degree.

Beside the above classification, it is common practice to differentiate between the stereoscopic recording approaches regarding to the field distortions into translation and angular displacement methods, see figure 3.3. In case of the translation method the light-sheet plane, the main plane of the lens and the image plane are parallel in relation to each other. As a result, the magnification factor is constant across the field of view (e.g. the image of a regular grid appears undistorted) and the image analysis varies only slightly from the analysis outlined in the previous chapter, see [44] for details. The drawback, on the other hand, is the decreased performance of this arrangement for increasing stereo opening angles. This happens because of optical aberrations and the decrease of the modulation transfer function towards the edges of the field of view (see also section 4.7) and because of the limited overlap of the observation areas of both cameras when the CCD sensor is not shifted with respect to the optical axis, see [44]. For these reasons the angular displacement method is usually applied where the light-sheet plane and the main plane of the lens intersect in a common line ${ }^{1}$. In this configuration the magnification factor varies across the field of view and typical distortions appear

\footnotetext{
${ }^{1}$ The translation imaging configuration can be seen as a special case of the angular-displacement arrangement with the line of intersection between the image plane, the main plane of the lens and the object plane at infinity.
} 

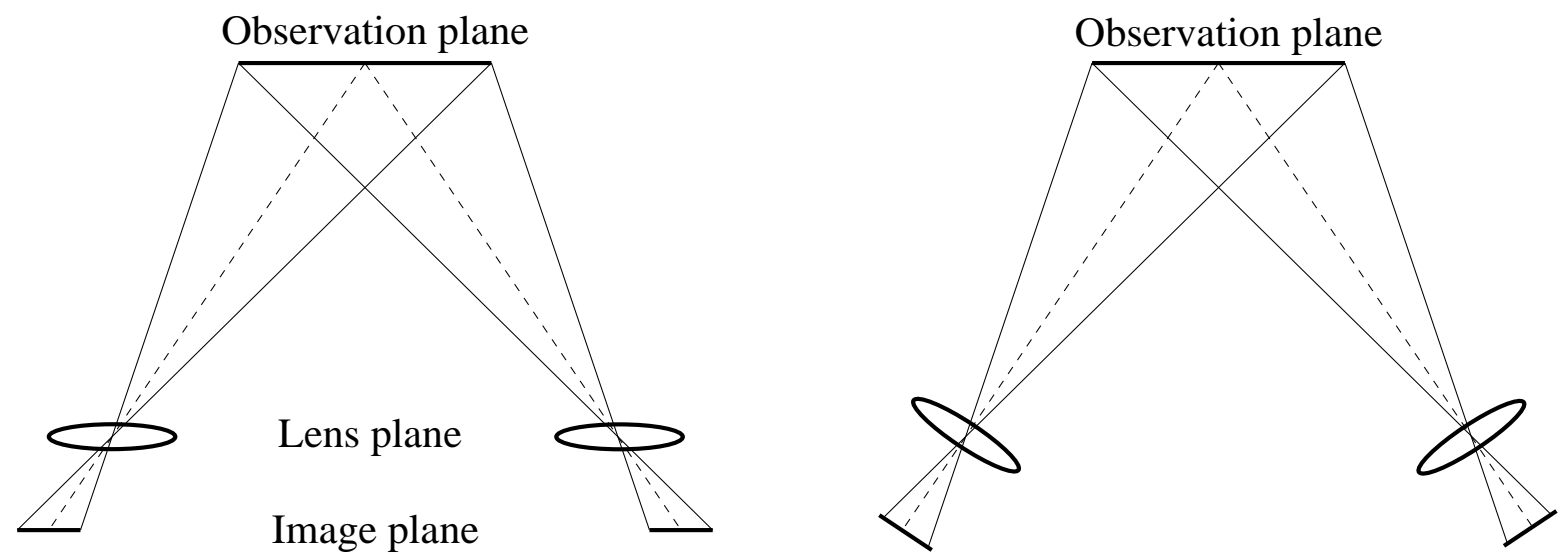

FIGURE 3.3: Stereo-scopic imaging configurations. Left: translation method. Right: angular displacement method.

as indicated in figure 3.4 for both camera-light-sheet arrangements shown in figure 3.2. The size, shape and location of the dark areas indicate the image of a rectangular area in the object space as a function of the camera arrangement. The difficulties associated with this effect and possible solutions will be further analysed in section 3.2.
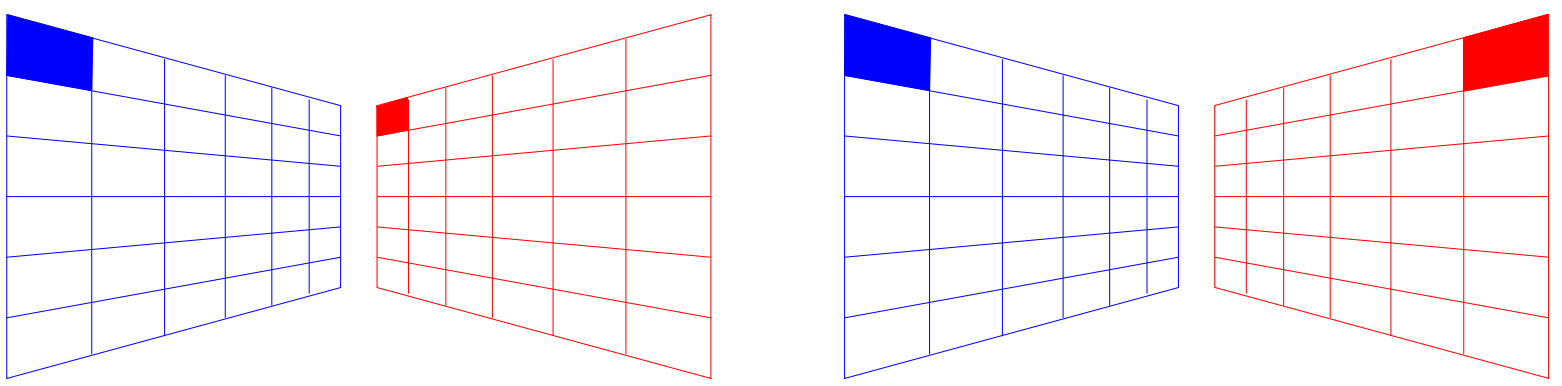

FIGURE 3.4: Linear field distortions of a regular grid due to the oblique observation directions for two angular displacement camera arrangements. Left: both cameras are located on the same side of the light-sheet according to the left drawing in figure 3.2). Right: cameras are separated by the light sheet (right drawing in figure 3.2).

\subsubsection{Error analysis}

It is obvious from our experience with the visual system of the human being, that the measurement error for the out-of-plane component and the accuracy of the perspective correction depend on the opening angle between the two cameras. Lawson and $\mathrm{Wu}$ have derived from geometrical considerations that for a symmetrical translation arrangement the relative out-ofplane error $\sigma_{\Delta z} / \sigma_{\Delta x}$ as a function of the off-axis position $x$ is given by

$$
\frac{\sigma_{\Delta z}}{\sigma_{\Delta x}}=\frac{1}{\sqrt{\left(x / d_{0}\right)^{2}+\left(h / d_{0}\right)^{2}}}
$$

where $d_{0}$ denotes the object distance and $2 h$ indicates the shortest distance between the lenses [71]. The upper left graph of figure 3.5 shows the variation of this relative out-of-plane error as a function of the off-axis position for various $x / d_{0}$ given by equation 3.1. The slope of 


\section{Stereo-scopic Particle Image Velocimetry}

the graphs implies that the relative measurement error can be significantly improved within $0<x / d_{0}<0.1$ by increasing the opening angle between the observation directions whereas for larger $x / d_{0}$ this effect becomes weaker.
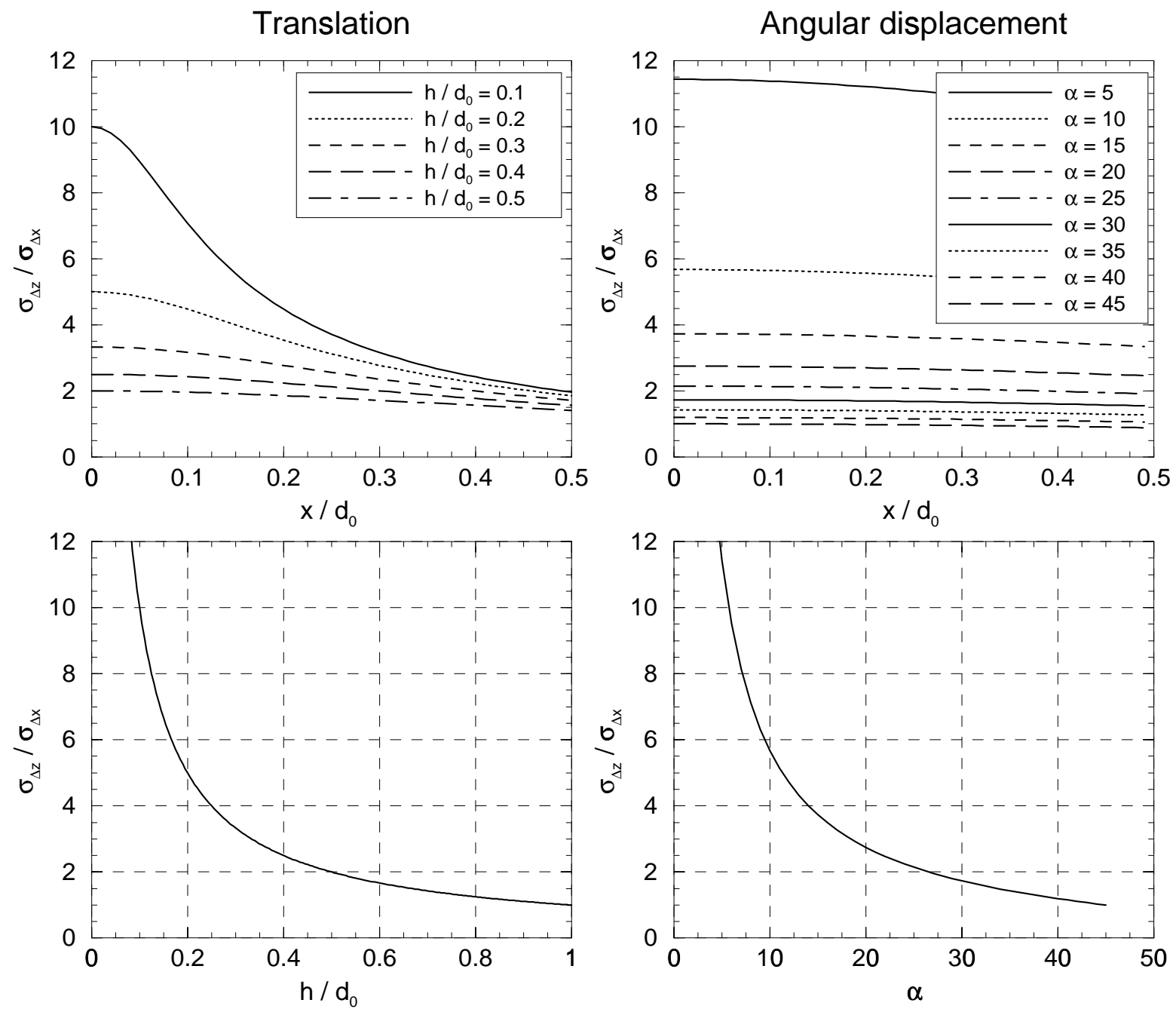

FIGURE 3.5: Upper graphs: Variation of this relative out-of-plane error as a function of the off-axis position for the translation method (left column) and the angular displacement arrangement (right column). Lower graphs: Dependence of the error for the principal observation rays from the viewing angle.

For the centre of the field of view $(x=0)$ the relative out-of-plane error as a function of the opening angle reduces to equation 3.2. This dependence, which gives the appropriate opening angle between the principle rays for a desired out-of-plane error, is shown in the lower left plot of figure $3 . .^{2}$

$$
\frac{\sigma_{\Delta z}}{\sigma_{\Delta x}}=\frac{1}{d_{0} / h}
$$

\footnotetext{
${ }^{2}$ As the performance of the stereoscopic approach decreases when the object distance is large with respect to the lens separation, the visual system of the human being changes from the stereoscopic approach to the interpretation of the perspective (relative magnitude of known objects and their position relative to each other - remote objects appear higher than close ones), shadow sizes, image contrast (absorption of light increases with increasing distance), degree of accommodation and other aids.
} 
In case of the angular displacement arrangement the variation of the relative out-of-plane error as a function of the $x$ coordinate is given by expression 3.3. Compared with the translation set-up, the dependence is rather weak as can be seen by comparing the upper plots of figure 3.5.

$$
\frac{\sigma_{\Delta z}}{\sigma_{\Delta x}}=\frac{\left[\cos ^{2} \alpha-\sin ^{2} \alpha\left[\left(\frac{x}{d_{0}}\right)^{2} \cos ^{2} \alpha\right]\right]^{\frac{1}{2}}}{\sin \alpha\left[1+\left(\frac{x}{d_{0}}\right)^{2} \cos ^{2} \alpha\right]^{\frac{1}{2}}}
$$

By substituting $x=0$ in equation 3.3 it turns out that the relative out-of-plane error at the optical axis is the reciprocal of the tangents of the off-axis angle $\alpha$, according to the following equation, and for $\alpha=45^{\circ}$ (opening angle $\theta=90^{\circ}$ ) the out-of-plane error $\sigma_{\Delta z}$ becomes comparable with the in-plane error $\sigma_{\Delta x}$ at the centre of the field of view, see lower right plot of figure 3.5 .

$$
\frac{\sigma_{\Delta z}}{\sigma_{\Delta x}}=\frac{1}{\tan \alpha}
$$

\subsubsection{Scheimpflug condition}

Unfortunately, large opening angles are often not feasible by using standard equipment due to the limited depth of focus, see section 4.7 for further details. For a typical configuration with $f=5.6, M=0.5$ and $\lambda=532 \mathrm{~nm}$, for example, the depth of focus is only $\delta_{z}=$ $2 f_{\#} d_{\text {diff }}(M+1) / M^{2} \simeq \pm 0.35 \mathrm{~mm}$. To overcome these difficulties, the conditions which improve the imaging when the object plane is tilted relative to the main plane of the lens, will be briefly derived.

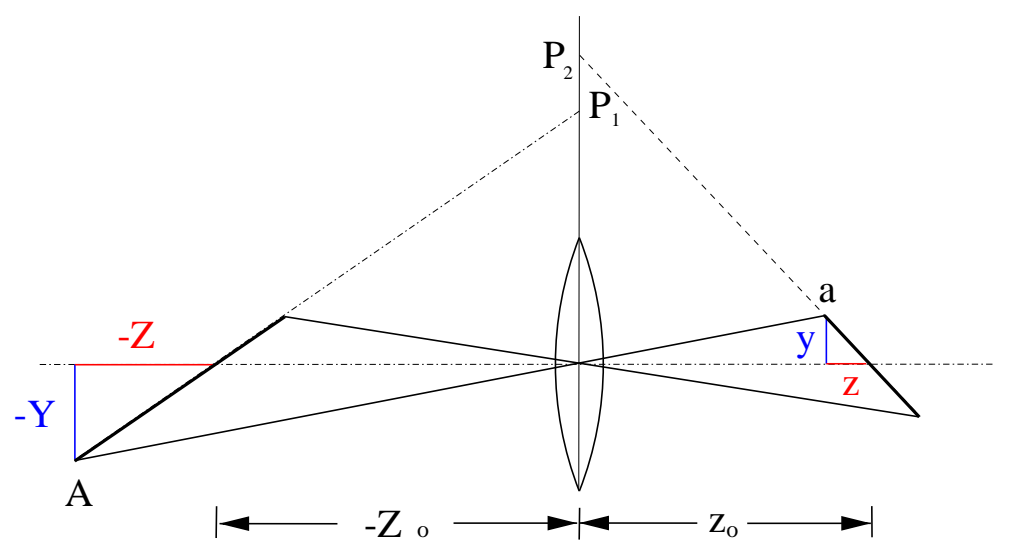

FIGURE 3.6: Scheimpflug condition: The image-, object- and main plane of the lens need to intersect in a common line for ideal imaging $\left(P_{1}=P_{2}\right)$.

Assuming that the main-plane of the lens intersects with the object- and image-planes at $P_{1}$ and $P_{2}$ respectively according to figure 3.6, it follows from geometrical considerations that the distance from the centre of the lens to the points of intersection can be expressed as $\overline{O P_{1}} / Z_{0}=Y / Z$ and $\overline{O P_{2}} / z_{0}=y / z .(-Y,-Z)$ denotes the coordinates of a non-axial objectpoint (measured from the intersection of the optical axis of the lens with the object plane) and $(y, z)$ are the corresponding image coordinates. Using the definition for the transversal 
magnification $M_{T}=y / Y=z_{0} / Z_{0}$ and the relation $z=M_{L} Z=M_{T}^{2} Z$ for the longitudinal magnification, it turns out that the two points of intersection coincide.

$$
\overline{O P_{2}}=\frac{z_{o} y}{z}=\frac{M_{T} Z_{o} M_{T} Y}{Z M_{T}^{2}}=\frac{Z_{o} Y}{Z}=\overline{O P_{1}}
$$

Thus the image-, object- and main plane of the lens need to intersect in a common line for ideal imaging. Although this relation was proven theoretically by the French mathematician Girard Desargues (1591-1661), and experimentally in 1901 by Jules Carpentier (Patent GB 1139/1901), this condition is named after the Austrian aerial cartographer Theodor Scheimpflug (1865-1911), who has derived this relations from the optical laws in his British Patent (GB 1196/1904) from 1904. However, it should be noted that this Scheimpflug condition is only a necessary condition which requires in addition that the object distance $d_{o}$ is larger than the focal length $f$ of the imaging system. For practical purposes this condition becomes significant for $\delta_{z} \leq L \tan \alpha$ where $\alpha$ denotes the angle between the optical axis of the lens and the light-sheet plane, $\delta_{z}$ is the depth of focus and $L$ is the size of the field of view. For small aperture or long focal length imaging, the adjustment of the image plane can be neglected for a wide range of opening angles between the two cameras in stereo-scopic imaging configuration.

\subsection{Evaluation of stereo-scopic image pairs}

The advantage of the angular-displacement technique with respect to the translation method is that optical aberrations as well as intensity losses, due to the decrease of the modulationtransfer function towards the edges of the field of view, can be neglected, see section 4.7. The inherent drawback, on the other side, is the characteristic variation of the magnification factor across the field of view due to the oblique viewing direction. Beside a variation of the spatial resolution across the field of view and a varying particle image density (number of particle image pairs per unit area) additional errors have to be taken into account, especially when both cameras are located on one side of the light-sheet. In this case, the size of each of a pair of measurement volumes considered for the calculation of the third velocity component is inversely proportional to each other according to figure 3.4.

\subsubsection{Determination of the mapping function}

For conventional PIV (single light-sheet single camera configuration) the variation of the magnification factor over the field of view is usually negligible because the image-plane and the main plane of the imaging system are parallel to the light-sheet. To ensure that the interrogation windows from each of a pair of stereoscopic recordings correspond to the same flow region and also, that the magnification is constant for all positions within the image, the distortions along with possible differences in the field of view have to be carefully determined before the line-by-line evaluation procedure, described in section 2.4, can be applied. For this process images of a regular calibration grid (or dot pattern) with a known line-spacing are usually acquired prior to the measurements while the calibration target must be perfectly aligned with the centre of the light-sheet planes in order to avoid systematic measurement errors, compare section 3.3. According to the procedure proposed in [108] each image is inverted and cross-correlated with an appropriate correlation mask ( + in the case of a calibration 
grid and $\bullet$ when a dot pattern is used) in order to determine the coordinates of the line crossing with sub-pixel accuracy ${ }^{3}$. After this step the imaging function between the image- and object plane can be changed from a discrete into a continuous representation by means of fitting a standard least squares surface to each of the image-object point sets, so that the first order projection matrix can be calculated for each observation direction along with the translation, rotation and magnification factor, [108].

$$
\begin{aligned}
& X=\frac{a_{11} x+a_{12} y+a_{13}}{a_{31} x+a_{32} y+1} \\
& Y=\frac{a_{21} x+a_{22} y+a_{23}}{a_{31} x+a_{32} y+1}
\end{aligned}
$$

In order to take into account aberrations of higher order and other non-linear distortions, the second order projection in form of equation (3.10) has to be applied. For this purpose the coefficients of the first order projection matrix have to be used as an initial estimate to a Levenberg-Marquart non-linear least squares fitting algorithm.

$$
\begin{aligned}
X & =\frac{a_{11} x+a_{12} y+a_{13}+a_{14} x^{2}+a_{15} y^{2}+a_{16} x y}{a_{31} x+a_{32} y+a_{33}+a_{34} x^{2}+a_{35} y^{2}+a_{36} x y} \\
Y & =\frac{a_{21} x+a_{22} y+a_{23}+a_{24} x^{2}+a_{25} y^{2}+a_{26} x y}{a_{31} x+a_{32} y+a_{33}+a_{34} x^{2}+a_{35} y^{2}+a_{36} x y} \\
a_{33} & =1
\end{aligned}
$$

As both sets of transformation equations describe just a mapping between two planar domains without any three-dimensional information, the location of the image with respect to the object has to be known in addition. This can be done either directly, by measuring the exact camera positions relative to the centre of the field of view, or indirectly by using the following set of equations, see [56] for mathematical details or [95] for the applicability in PIV.

$$
\begin{aligned}
X & =\frac{a_{11} x+a_{12} y+a_{13} z+a_{14}}{a_{31} x+a_{32} y+a_{33} z+a_{34}} \\
Y & =\frac{a_{21} x+a_{22} y+a_{23} z+a_{24}}{a_{31} x+a_{32} y+a_{33} z+a_{34}}
\end{aligned}
$$

The appearance of the $z$-coordinate requires that the calibration procedure has to be repeated for different $z$ locations in order to determine all unknown coefficients. This is especially useful when the position of the cameras is not accessible or for applications in water, where the air-glass-water interface has to be taken into account.

\subsubsection{Image warping}

Once the reconstruction coefficients have been properly determined, the transformation equations can be applied to deform each acquired, single-exposed image in such a way that the magnification factor is constant across the back-projected image and the field of view is identical for all acquired images. Using this technique optical parameters such as the focal length

\footnotetext{
${ }^{3}$ As this method is quite time consuming, Hough transformation methods are usually applied in this thesis to find the coordinates of the line crossings, see [18].
} 


\section{Stereo-scopic Particle Image Velocimetry}

and the magnification factor never need to be determined, and non-linear distortions introduced by the transparent test-section wall or other optical components in a non-collimated beam can be accounted for, when the transformation equations are extended to higher order. Furthermore, the simplified stereo-equations can be applied to calculate one three-component displacement field from a pair of two-component sets as the magnification factor is constant.

$$
\begin{aligned}
\Delta X & =\frac{\Delta x_{2} \tan \alpha_{1}+\Delta x_{1} \tan \alpha_{2}}{\tan \alpha_{2}+\tan \alpha_{1}} \\
\Delta Y & =\frac{\Delta y_{2} \tan \beta_{1}+\Delta y_{1} \tan \beta_{2}}{\tan \beta_{2}+\tan \beta_{1}} \\
\Delta Z & =\frac{\Delta x_{1}-\Delta x_{2}}{\tan \alpha_{2}+\tan \alpha_{1}}
\end{aligned}
$$

As the grey-level values are only defined at integer pixel locations within the image, a spatial transformation based on equation (3.6) to (3.11) causes a mapping into locations for which no grey levels are defined. Thus, it becomes necessary to infer what the correct grey-level values at those locations should be, based on the grey-level values at integer pixel coordinate locations. To validate the performance of different image interpolation methods, two particle image fields, created from one measured (or simulated) single exposed grey-level pattern by using the transformation coefficients $\left(a_{11}=0.999, a_{22}=1\right.$, other coefficients zero) for the first image and ( $a_{11}=1, a_{22}=1$, other coefficients zero) for the second image, where crosscorrelated [39]. As the true displacement linearly varies by two pixels over a range of 2000 pixels (reference line in figure 3.7) a correlation between the two back-projected images will yield information about artefacts due to non-properly chosen sub-pixel increments (number of grid points per pixel considered for calculating the grey-value in the back-projected image) or due to interpolation procedures which have been chosen improperly. The simplest schemes
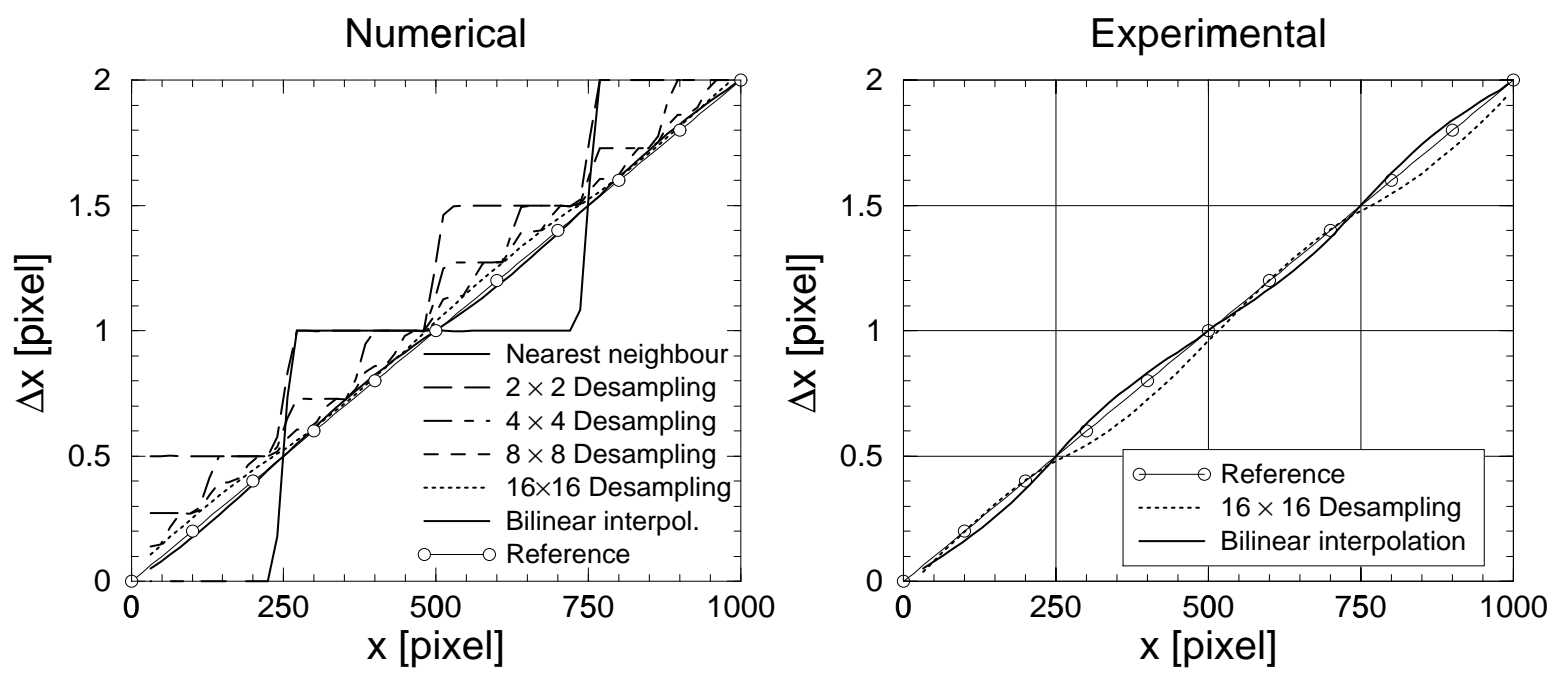

FIGURE 3.7: Systematic deviation of the measured displacement $\Delta x$ for different interpolation schemes. A linearly varying particle displacement appears as a step function after the image de-warping has been performed whereby the step-size decreases with increasing performance of the image interpolation scheme.

based on nearest-neighbour approach are easy to implement but undesirable artefacts like 
distortions can be hardly avoided and systematic errors appear as indicated in figure 3.7. The so called de-sampling method yield better results, provided each pixel is subdivided in at least four sub-pixel or the bilinear interpolation where the grey levels of the four nearest integral neighbours of a non integral coordinate are consulted to determine the appropriate value. More sophisticated approaches like fitting a $\sin x / x$ type surface through a much larger number of neighbours (cubic convolution interpolation method) yield much smoother results but at the cost of computational time. For this reason the bilinear approach was applied for the analysis of the results shown in chapter 5 to 7 .

\subsubsection{Vector field warping}

As the proper de-warping in the image space is time consuming and requires a redistribution of the original images, which does not increase confidence ${ }^{4}$, the de-warping can be performed in the vector space as soon as the conventional line-by-line interrogation procedure has been applied. Using this approach the equally spaced grid points in the object space are transformed into the image space according to the transformation equations (3.6) and (3.7), and the velocity at each image grid point is calculated by means of linear interpolation. In order to minimise or avoid the interpolation procedure, the acquired images can either be evaluated with a smaller step-size or interrogated at the proper positions given by the grid points in the object space.

The inherent drawback of this approach is the fact that the magnification factor varies over the field of view as well as the measurement error and the detectability [51, 52]. Furthermore, if the camera system is symmetrical and located on one side of the light-sheet (left image in figure 3.4), the interrogation windows from each of a pair of stereoscopic images backprojection in the physical space may differ substantially in size, which means that in effect different flow volumes are considered for the calculation of the third velocity component ${ }^{5}$. The significance of this statement will be considered in the section 3.3. For applications in large wind tunnels the problem is further increased due to unequal object distances or nonsymmetric stereoscopic PIV set-ups.

\subsubsection{Interrogation window warping}

In order to avoid any modification of the measured grey-level distribution itself and to be independent of interpolation algorithms in the vector space, an alternative approach can be implemented based on a local distortion of the size and shape of the interrogation window in such a way that the number of particle images remains constant over the field of view and the shape varies in a way that the back-projection of the local interrogation areas into the object space is always the best approximation to a square or other desired shapes ${ }^{6}$. This can be easily done either by calculating a direct correlation, which is not restricted to radix-2 sized interrogation window dimensions with rectangular shape as the conventional FFT-based

\footnotetext{
${ }^{4}$ An initially circular particle image for example may appear elliptically after the transformation has been performed. This artifact can bias or lower the principal measurement accuracy.

${ }^{5}$ In the case of a symmetrical setup with a light-sheet between the two cameras (dashed configuration in figure 3.2 and right image in figure 3.4) the fluid elements considered for the calculation of the third velocity component are equal in size but the spatial resolution remains a function of the image location.

${ }^{6}$ The information concerning the position, size and shape of the interrogation window is known from the calibration grid analysis simply by substituting all desired values of the object plane into the equations and using a nearest neighbour approach to find the appropriate position in the image plane.
} 


\section{Stereo-scopic Particle Image Velocimetry}

correlation algorithm [109], or by using the FFT-based free-shape cross-correlation which combines the advantages of the direct correlation (free-sized and free-shaped windows, high accuracy) and the widely used FFT-based correlation (high speed evaluation) [88]. Although the computational time is increased by using this evaluation technique the total computational time is comparable to the de-warping technique as the time consuming image de-warping can be avoided and multi-pass techniques are not required. Due to the discrete nature of the images the interrogation windows from each of a pair of stereoscopic images will never be exactly identical but due to the statistical approach small differences do not affect the result.

\subsection{Calibration validation}

To ensure that the interrogation windows from each of a pair of stereoscopic images correspond to the same region of flow, the properties of the imaging must be constant, when the measurements take place, and identical with the calibration condition. Unfortunately, interrupting the experiment and entering the test section of a wind-tunnel for the calibration procedure may lead to different boundary conditions, and mechanical or thermal variation during the experiment can be hardly avoided, especially in industrial environments. To demonstrate the effect of non properly aligned calibration grids or poorly performed evaluation, let us suppose that the displacement fields from the left and right camera are identical apart from a phase factor and similar to the streaky flow pattern as present in the near wall region of turbulent flows.

$$
\begin{aligned}
& \Delta x_{1}=\sin (x) \\
& \Delta x_{2}=\sin (x+\phi)
\end{aligned}
$$

By changing the phase $\phi$ the effect of a simple translation between both images can be simulated while $\phi=0$ corresponds to zero out-of-plane displacement according to equation 3.14. Figure 3.8 shows the artificially introduced out-of-plane displacement for $\phi=$

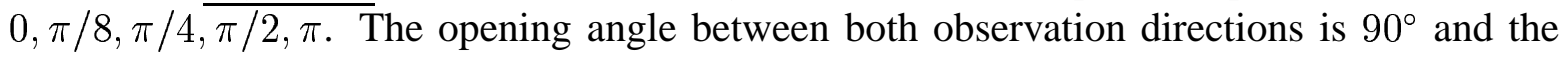
object distance is assumed to be large so that the perspective error is negligible.

FIGURE 3.8: Artifical out-of-plane displacement introduced by a non properly aligned cal-

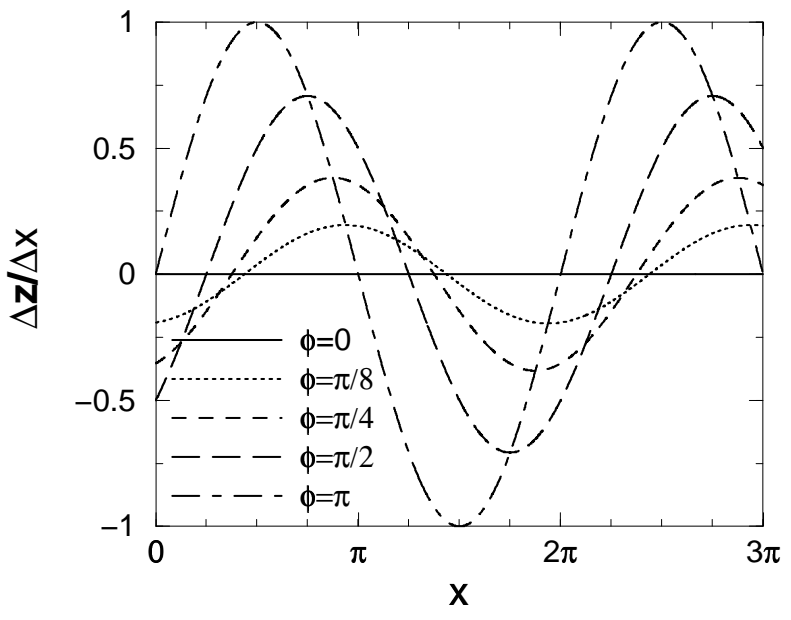
ibration grid or poorly performed evaluation.

It can be seen that the error increases with increasing misalignment and becomes comparable to the true in-plane displacement for $\phi=\pi$ so that the misalignment can be easily detected 
by means of fluid-mechanical considerations. This is almost impossible for small $\phi$, especially when the relation between the components possesses some obvious symmetry which may focus the attention in the wrong direction. This is clearly visible in figure 3.9 where a Rankine vortex with a tangential particle image displacement of \pm 4 pixel is displayed. The $512 \times 512$ pixel images have been analysed with $32 \times 32$ pixel interrogation windows, each of them containing approximately 25 particles generated at random positions within the Gaussian shaped light-sheet of finite thickness. The curvature of the particle trajectory is taken into account, such that particle images close to the core of the vortex actually orbit the core at the same radius but centrifugal forces are not considered. Before the analysis has been performed the simulated particle image pattern has been duplicated and properly shifted in opposite directions by choosing the values of the coefficient $a_{13}$ and $a_{23}$ and the sub-pixel increment properly. The opening angle between the assumed observation directions is again $90^{\circ}$ and the object-distance 10000 pixel (20 times the field of view) to avoid projection effects.
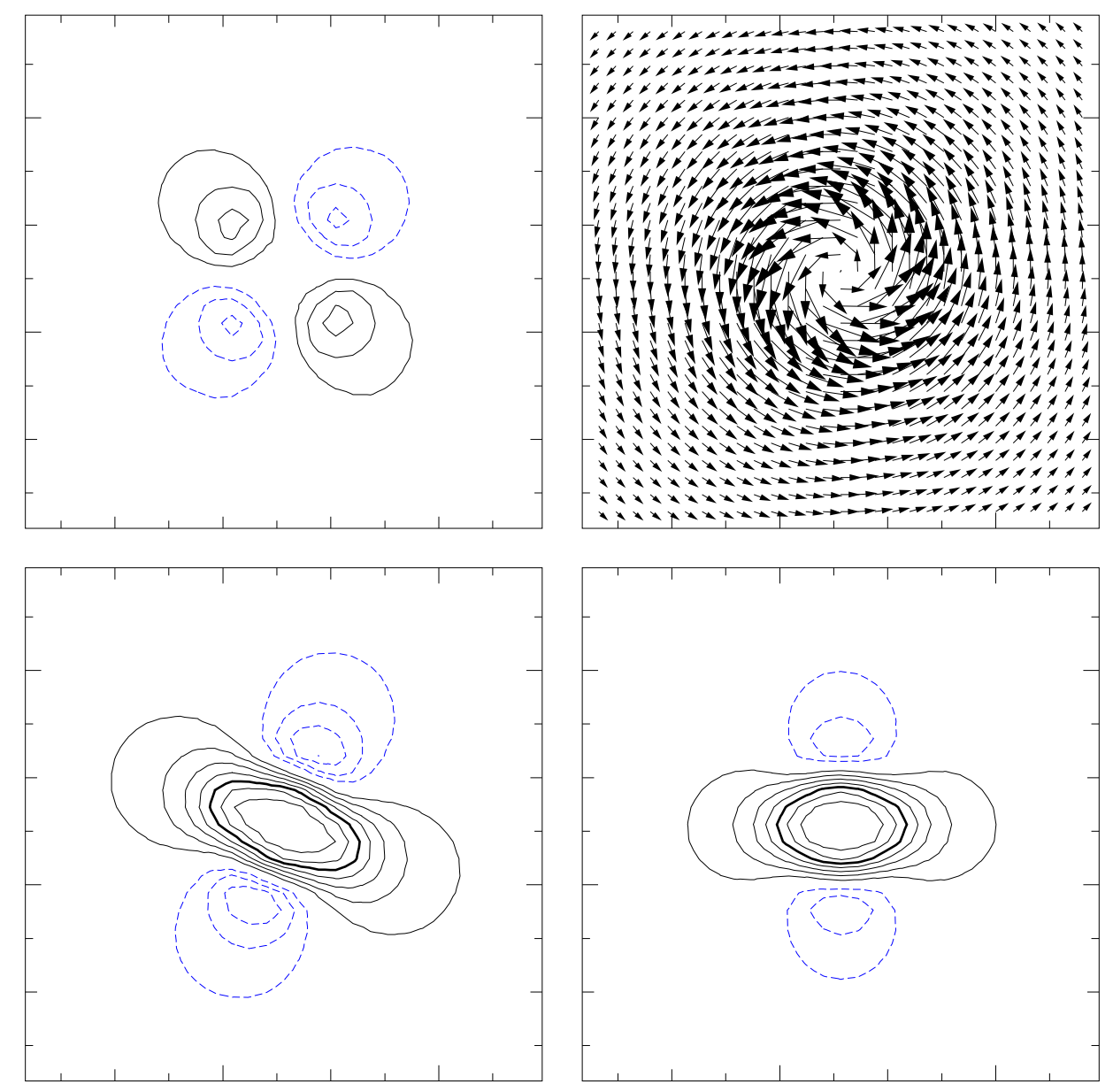

FIGURE 3.9: Spatial distribution of the out-of-plane displacement introduced by non properly aligned calibration grid or poorly performed evaluation (average over 20 fields). The line spacing in the contour plots is incremented in steps of 0.1 pixel and different line styles indicate different out-of-plane directions.

The range of the out-of-plane displacements as a function of the in-plane shift depends on both, magnitude and direction and can be quite large with respect to all other error sources in PIV according to table 3.1. Figure 3.9 shows the spatial distribution of the error. The shift is 
$\Delta x=16$ pixel for the upper left image, $\Delta y=16$ pixel for the lower right and 16 pixel in each direction for the lower left sample. A surprising result is the completely different symmetry as only the displacements in $x$-direction account for the out-of-plane component. This explains the different $\epsilon_{z}$ for identical shifts in orthogonal spatial direction, see table 3.1.

TABLE 3.1: Artificial outof-plane displacements $\epsilon_{\mathrm{Z}}$ as a function of the magnitude and direction of the in-plane

\begin{tabular}{llc}
\hline \multicolumn{2}{c}{ In-plane shift [pixel] } & Out-of-plane error [pixel] \\
\hline$\Delta x=8$ & $\Delta y=0$ & $-0.520 \leq \epsilon_{z} \leq 0.482$ \\
$\Delta x=16$ & $\Delta y=0$ & $-0.688 \leq \epsilon_{z} \leq 0.705$ \\
$\Delta x=32$ & $\Delta y=0$ & $-1.049 \leq \epsilon_{z} \leq 1.109$ \\
\hline$\Delta x=8$ & $\Delta y=8$ & $-0.576 \leq \epsilon_{z} \leq 1.147$ \\
$\Delta x=16$ & $\Delta y=16$ & $-0.824 \leq \epsilon_{z} \leq 1.526$ \\
$\Delta x=32$ & $\Delta y=32$ & $-1.120 \leq \epsilon_{z} \leq 2.076$ \\
\hline$\Delta x=0$ & $\Delta y=8$ & $-0.436 \leq \epsilon_{z} \leq 1.076$ \\
$\Delta x=0$ & $\Delta y=16$ & $-0.602 \leq \epsilon_{Z} \leq 1.611$ \\
$\Delta x=0$ & $\Delta y=32$ & $-0.969 \leq \epsilon_{z} \leq 2.049$ \\
\hline
\end{tabular}

To validate the boundary conditions a cross-correlation between the de-warped single exposed images from the camera pair in stereoscopic imaging configuration, acquired at the same instant of time, has to be calculated in order to obtain the local misalignment between the images. As the illuminated particles are almost identical, especially for pure forward, backward and $90^{\circ}$ degree observation direction ${ }^{7}$, and their different image position has been corrected by the applied warping, based on the analysis of the calibration grid, the output must be a zero-displacement field. Any serious misalignment due to translation, rotation or deformation (magnification change of one image) will become obvious in the displacement field and can be corrected by calculating the mapping function between both samples and combining the coefficients of this transformation with the coefficients determined with the calibration grid analysis [44]. This non-intrusive approach, based on the particle image fields itself, can be applied to each acquired image pair to guarantee that everything involved in the measurement is unaffected by wind tunnel vibrations, thermal distortions e.g. for the duration of the measurement [44]. This becomes important in noisy environments or for long acquisition times. Unfortunately, an equal displacement of both cameras can not be detected by using this approach, except if a mark is present in both images such as a bright model reflection or a tiny dark shadow within the light-sheet.

\footnotetext{
${ }^{7}$ Due to the finite aperture of the imaging system this approach is also feasible for non symmetrical observation directions or forward/backward configurations but the quality is slightly reduced due to possible unpaired particle images.
} 


\section{Multiplane Stereo Particle Image Velocimetry}

The Stereo-scopic Particle Image Velocimetry (PIV) described in the previous chapter has become a widely used technique for investigations where the spatial distribution of the velocity is needed to understand the flow physics. Unfortunately, the distribution of the velocity within one single plane, captured at one instant in time, does not yield always the information required to answer fluid-mechanical questions. In case of turbulent flows, for example, spacetime correlations together with the spectrum of the fluctuations are important in the statistical theory of turbulence as there is a link between these quantities and the decomposition of the flow field into a series of harmonic modes. For the identification of the most energetic velocity structures, the proper orthogonal decomposition is a well suited technique but in general it requires spatial and temporal information about the flow field. The eigenvalues of the velocity gradient tensor are also increasingly used to describe various flows because these quantities play a role in the theory of dynamical systems and topology. The vorticity vector (all components) is another important quantity due to its Galilean invariance as well as the acceleration in its Lagrangian and Eulerian form in order to study the formation and interaction processes of moving flow structures.

In order to retrieve the desired information about the spatio-temporal flow unsteadiness other sophisticated imaging techniques have been developed such as the phase-conjugate holographic system, the scanning light-sheet PIV, or the $3 D$ particle tracking technique $[5,10$, 101]. Despite the high level of development of each cited technique the information about the spatial-temporal flow unsteadiness remains limited and for applications in large wind tunnels where acquisition time, optical access and observation distances are constrained, their implementation remains challenging due to their complexity, costs and manpower demand [30].

To overcome these limitations a stereoscopic PIV based technique has been developed which is well suited to determine many fluid-mechanical quantities with high accuracy and spatial resolution $[45,39,46]$. This technique is reliable, robust and easy to handle. Furthermore it is based on standard PIV equipment and evaluation procedures so that available PIV systems can be easily extended. In the following sections this technique will be referred as multiplane stereo PIV in order to emphasise its flexibility in spatio-temporal light-sheet positioning dependent on the value in question.

\subsection{Principles}

The multiplane stereo PIV system, developed for applications in air flows, consists of a fourpulse laser system delivering orthogonally polarised light, two pairs of high resolution progressive scan CCD cameras in an angular imaging configuration with Scheimpflug correction, 
two high reflectivity mirrors and a pair of polarising beam-splitter cubes, each consisting of two cemented right-angle prisms with an appropriate interference coating in between, see figure 4.1.

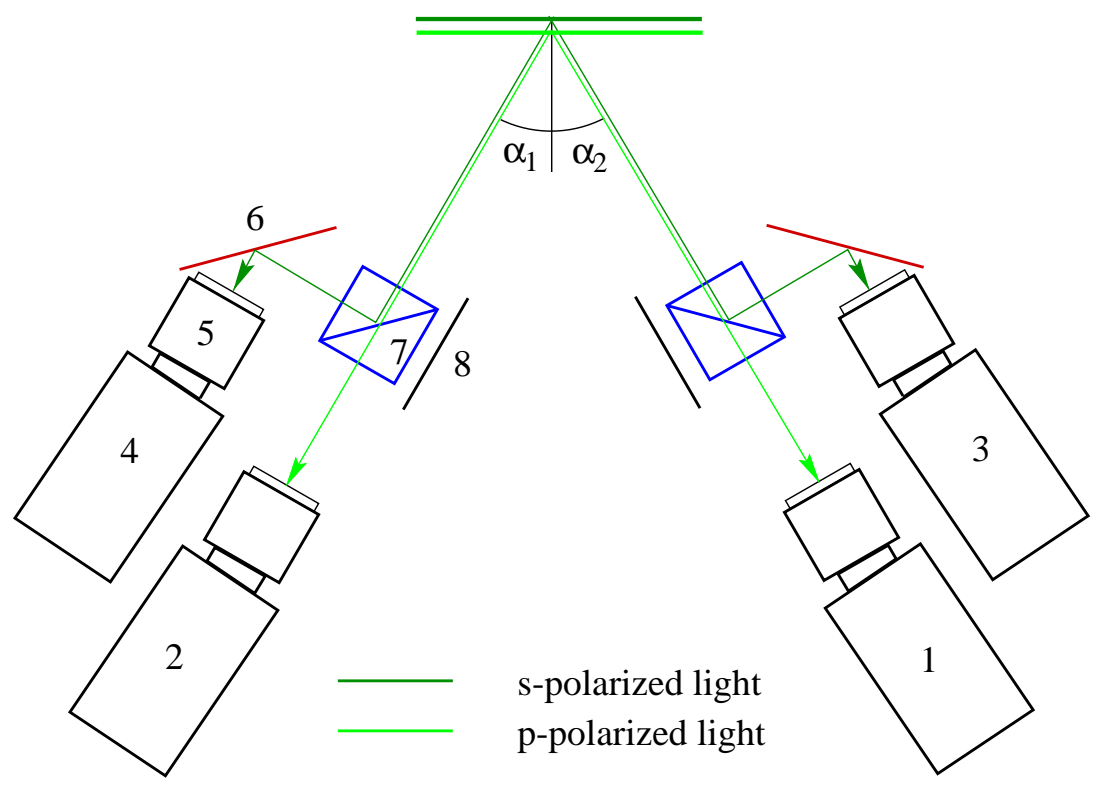

FIGURE 4.1: Schematic setup of the recording system. 1-4 digital cameras, 5 lens, 6 mirror, 7 polarising beam-splitter cube with dielectric coating between the two right-angle prisms, 8 absorbing material, $\alpha$ opening angle.

After the illumination of the tracer particles with orthogonally linearly polarised light, the polarising beam-splitter cube (7) separates the incident wave-front scattered from the particles into two parts. The light which passes straight through the cube emerges linearly polarised with the plane of the electric field vector parallel to the plane of incidence defined for the multi-layer film (p-polarised). The light emerging from the cube at right-angles to the incident wave front (having been reflected inside the cube at the dielectric multi-layer film) is oriented orthogonally to the plane of incidence (s-polarised). The separation based on polarisation works perfectly as long as the radius of the spherical particles is comparable to the wavelength of the laser light and the observation direction is properly aligned to the direction of the polarisation vector [8]. In order to avoid contrast reduction in the image plane due to background light emerging from the opposite beam-splitter-camera system, one surface of each cube is covered with an absorbing material (8).

Before entering the lens the s-polarised light coming from the polarising beam-splitter cube is reflected to achieve identical orientation of the image plane for all cameras. This simplifies the matching of the four observation areas and saves computing time during the evaluation of the images. The polarisation characteristics of these mirrors is not important as only the intensity of the scattered light is needed after the polarising beam-splitter cube has been passed.

The orthogonally linearly polarised light emerging from the polarising beam-splitter cube is finally recorded by means of high resolution progressive scan CCD cameras in an angular imaging configuration. Due to the oblique viewing direction and the limited depth of focus of the lens, the image plane, the main plane of the lens and the object plane need to intersect in a common line (Scheimpflug condition) according to section 3.1.2, in order to obtain 
focused particles everywhere within the image plane. Therefore, each camera lens must be connected to a specially designed one-axis tilt-adapter (Scheimpflug-adapter). The axis of rotation should coincide with the centreline of the CCD sensor to ensure that all particle images along this axis remain in focus under rotation. This simplifies the installation of the system and the focusing process since the image location of the centreline in object space and the opening angle between corresponding camera pairs remain constant under Scheimpflug adjustment. For magnification and field of view adjustments (necessary for maximising the amount of stereo information) all Scheimpflug-adapters should be mounted on a two-axis linear translation stage which allows high precision translations by thumb screws. In order to simplify the adjustment procedure without restricting the flexibility of the system the left and right recording systems can be connected to different base-plates with individual rotation stage.

\subsection{Four-pulse-laser System}

For the illumination of the tracer particles the beams of four independent laser-oscillators need to be combined in such a way that the linearly polarised light-sheets can be positioned independently with respect to each other. This can be easily and precisely done by the fourpulse system shown in figure 4.2. The lasers consist of a Neodymium-Yttrium-AluminiumGranat rod embedded in an unstable resonator with a variable reflectivity output mirror (the transmission decreases from the centre to the edge in order to eliminate the intensity maxima of higher orders introduced by the unstable resonator) and a white light flash-lamp for the excitation of the crystal-atoms [60]. The monochromatic unpolarised radiation spontaneously emitted by the laser material (1) immediately after the excitation of the atoms will be linearly polarised when it emerges from the dielectric Glan-Laser polariser (6) and circularly polarised behind the retardation plate (5) which consists of a plane-parallel slice cut out of a uniaxial crystal such that the cut-plane is parallel to the crystalline optic axis. The thickness of the plate is such that the phase difference (retardation of the slow ray in comparison to the fast ray at emergence) is $\lambda / 4$ for normal incident and a given wavelength (here $\lambda=1064 \mathrm{~nm}$ ). The direction of propagation of the circularly polarised beam is reversed by reflection in a mirror (2) and thus the sense of the circular polarisation as well (due to the $180^{\circ}$ phase shift induced by the mirror) before the second transformation into linearly polarised light. Thus, the linearly polarised wave of the reflected beam, as it finally emerges from its second path through the retarder (5), is orthogonal to the incident linearly polarised wave and can be rejected by means of the dielectric polariser (6) constructed from an air-spaced right-angle prism pair with parallel optical axis. In this configuration the polariser quarter-wave plate combination acts as an isolator (or closed switch) when the angle between the plane of linear polarisation and the crystalline optical axis equals exactly either $\pm 45^{\circ}$ (for other angles the transformation is from linear to elliptical polarisation due to the different amplitudes of the ordinary and extraordinary beam behind the retardation plate). For the stimulated emission of radiation the electro-optical block (4) can be made equivalent to a quarter-wave plate by appropriate choice of block length, bias voltage and suitable orientation with respect to the quarter-wave plate such that the beam can pass the Glan-Laser polariser when the population inversion reaches its maximum. Using this optical arrangement the laser power can be increased by reducing the pulse duration and the output energy of the laser is adjustable by changing the time delay between the flash-lamp and the Pockels cell bias voltage.

In figure 4.2 the linearly orthogonally polarised infra-red beams released by the resonator 
pair are combined using a dielectric polariser at the Brewster angle (8a) which reflects the p-polarised light partially at every dielectric interface within the multi-layer coating while transmitting the s-polarised light with almost no reflection. A retardation plate behind the Brewster window (5) transforms both linearly polarised beams into circularly polarised light before they enter a properly cut and temperature stabilised highly doped KD*P (Kaliumdihydrogenphosphat) crystal (10) for polarisation selection and generation of the second harmonic $(532 \mathrm{~nm})$ from the fundamental wavelength $(1064 \mathrm{~nm})$, see [70] for details. To separate the two wavelengths, a high energy harmonic separator (9) is used consisting of a specially coated substrate which reflects the harmonic $\left(R_{\max }\right.$ at $\left.532 \mathrm{~nm}\right)$ and transmits the fundamental $\left(T_{\max }\right.$ at $1064 \mathrm{~nm}$ ) wave. As the linearly polarised light emerging from the frequency doublers possesses the same state of polarisation, a $\lambda / 2$ retardation plate (11) has to be inserted before the superposition of the four beams by means of another dielectric polariser (8c) can take place. As the orientation of the frequency doubler (10) affects the efficiency of the second harmonic generation and may change the angle between the incoming and outgoing beam, problems such as different output energy may occur when using a system where two beams pass the same frequency doubler crystal.

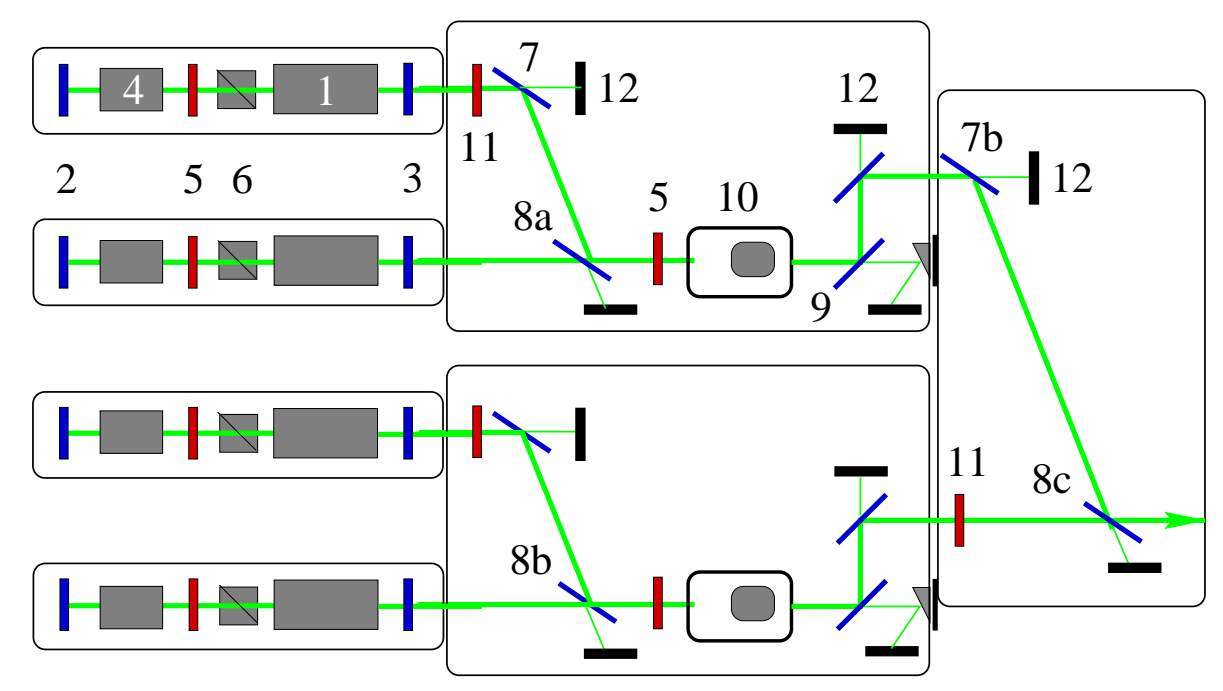

FIGURE 4.2: Four-pulse four frequency doppler laser system. 1 Pump cavity, 2 Full reflective mirror, 3 Partially transmitting mirror, 4 Pockels cell, $5 \lambda / 4$ retardation plate, 6 Glan-Laser polariser, 7 Mirror, 8 Dielectric polariser, 9 Dichroic mirror, 10 Frequency doubler crystal with phase angle adjustment, 11 $\lambda / 2$ retardation plate, 12 Beam dump.

An alternative system which has been tested as well is shown in figure 4.3. The recombination takes place behind the frequency doubler in the visible wavelength range so that each beam energy can be optimised independently by appropriate orientation of the frequency doubler crystal. The superposition can be adjusted again using the dielectric polariser 8c. As the light from each laser pair is linearly orthogonally polarised the Q-switches of the outer oscillator pair in figure 4.3 are connected with a Pockels cell (4) in the four beam combination optics. When these oscillators deliver the light, the corresponding Pockels cell is switched simultaneously and turns the state of polarisation of the incident beam by an angle of $90^{\circ}$. The advantage of the laser system shown in figure 4.3 lies in its ability to maximise the output energy for each laser independently. The price is two extra frequency doublers and Pockels cells and slightly more complicated combination optics. In addition as the beams take the same 
path behind the first dielectric polariser (8a,b), but need different Pockels cell voltage for exit, the cavities of each laser pair cannot be fired simultaneously. This is usually not a restriction, as only orthogonally polarised light needs to be delivered simultaneously for multiple plane stereo recording.

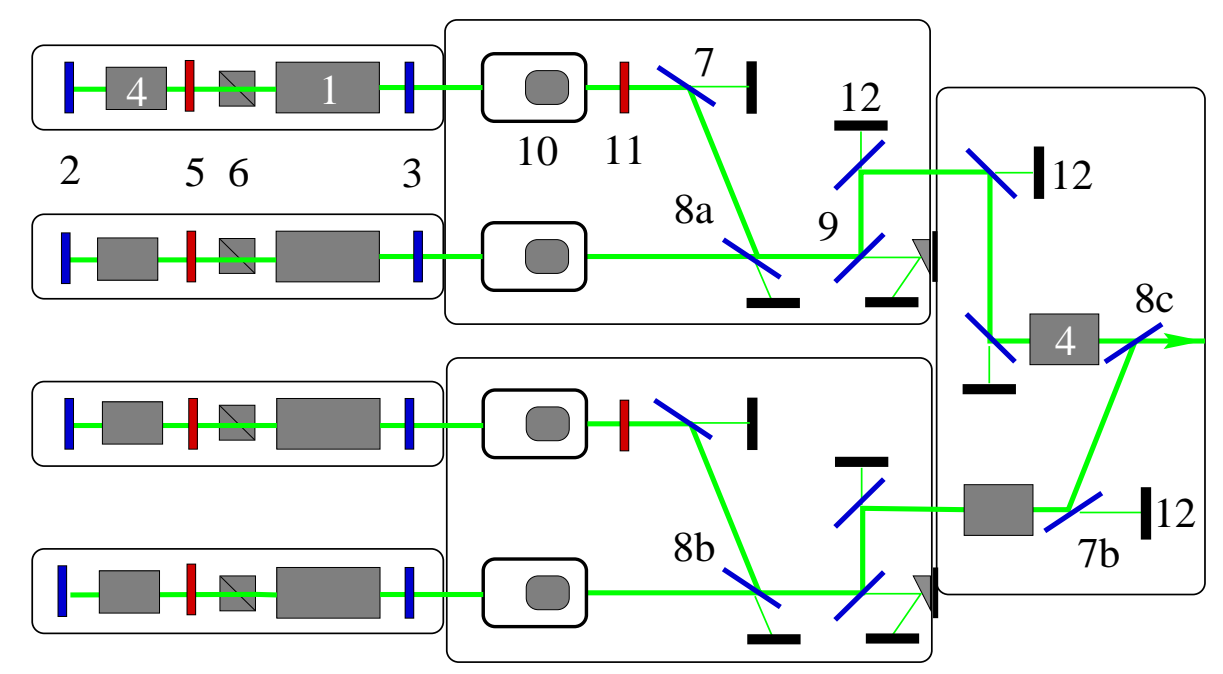

FIgURE 4.3: Four-pulse laser system. 1 Pump cavity, 2 Full reflective mirror, 3 Partially transmitting mirror, 4 Pockels cell, $5 \lambda / 4$ retardation plate, 6 Glan-Laser polariser, 7 Mirror, 8 Dielectric polariser, 9 Dichroic mirror, 10 Frequency doubler crystal with phase angle adjustment, $11 \lambda / 2$ retardation plate, 12 Beam dump.

\subsubsection{Performance of spatial light-sheet separation}

The appropriate method of adjusting the displacement between the orthogonally polarised light-sheets depends on the desired distance. Small separations between the orthogonally polarised light-sheet pairs (up to a few millimetres) can be generated by a simple rotation of mirror $8 \mathrm{c}$ in the re-combination optics around the axis perpendicular to the laser-beam plane. This is possible as the divergence between the s- and p-polarised beams is negligible when the separation is small with respect to the distance from the measurement position to mirror 8c. A translation of mirror $8 \mathrm{c}$ on the other hand cannot be applied in order to separate the orthogonal polarised beams as the focal lens in the light-sheet optics will superimpose parallel rays at the focal point. The relative separation between the beams can be easily controlled by means of a target located at the measurement position which is slightly tilted with respect to the light-sheets in order to increase the resolution.

For a wider range of light-sheet spacings (up to a few $\mathrm{cm}$ ) and independent positioning of both beam-pairs, it is useful to remove mirror $7 \mathrm{~b}$ along with the beam dump (12) such that two spatially separated laser beams with orthogonally polarised radiation emerge. Using two separate light-sheet-optics (one for each polarisation) each with a $45^{\circ}$ mirror behind the last lens, all positions are possible by moving the mirrors. Once calibrated, the actual position of each pair of light-sheets is given by a micrometer scale. For the conservation of the polarisation the mirror needs a dielectric broad-band coating optimised for $532 \mathrm{~nm}$ and $45^{\circ}$ angle of incidence to provide a $99 \%$ reflectance in both s- and p-planes also when the angle is not exactly $45^{\circ}$. In addition, a high damage threshold ( $>10 \mathrm{~J} / \mathrm{cm}^{2}$ for $15 \mathrm{~ns}$ pulse duration) is 
necessary to minimise the probability of laser induced damage. Due to the thermal sensitivity of the laser material and the unstable resonator, the previously mentioned adjustments should be performed under thermal equilibrium conditions.

\subsubsection{Generation and controlling of the timing sequence}

In order to trigger all necessary components quickly and easily a 16 channel sequencer-board with an output frequency between $0.01 \mathrm{~Hz}$ and $1 \mathrm{MHz}$ has been used. The width of each pulse is freely adjustable according to the specifications of the used equipment. The delay of all output trigger pulses with respect to the input trigger can be selected in the range from $50 \mathrm{~ns}$ up to $140 \mathrm{~s}$ with a resolution of $50 \mathrm{~ns}$ (12.5 ns jitter). Figure 4.4 shows the user-interface for the generation and operation of the appropriate timing-sequence.

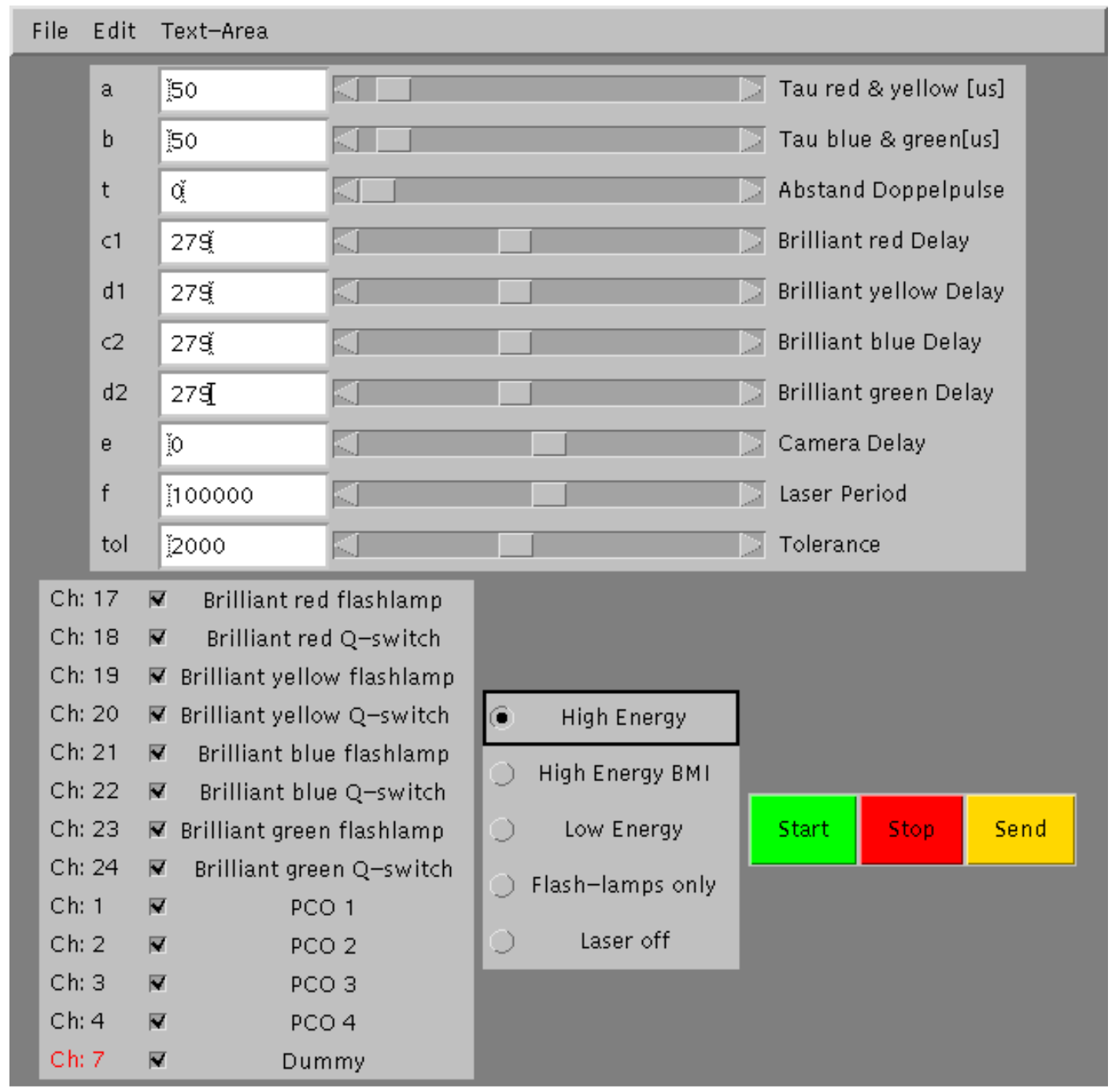

FIGURE 4.4: Window-interface to select and control the laser- and camera-timing

The first two sliders located at the top of the window control the pulse separation for both laser pairs separately whereas the third one determines the time-interval between the first pulses of each laser pair. Thus, all pulse combinations described in section 4.3 and 4.4 
for multiple-plane recording can be easily generated. Slider 4 to 7 from the top specify the delay between the flash-lamps and the Pockels-cells in order to adjust the output energy of each laser individually and with slider 8 the time for the read-out of the first camera images can be adjusted with respect to the first laser pulse. In the centre menu in the lower half of figure 4.4 the specifications of the laser-system (Quantel, BMI) can be chosen as well as the mode of operation (high or low energy, flash-lamps only to keep the laser material at the appropriate temperature, laser off). All other possibilities like cameras only (for field of view adjustments) or cameras together with only one oscillator (for checking the separation by means of the polarisation) can be selected in the lower left menu by a mouse click on the right bottom. After selecting the appropriate set of parameters the sequence has to be sent to the electronic board and can be activated using the start bottom at the lower right of figure 4.4 and afterwards terminated with the neighbouring stop bottom. This user-friendly interface enables the user to perform all alignments and calibrations within reasonable time.

\subsection{Modes of Operation I - In-plane flows}

Once installed the multiplane stereo system is well suited to determine different fluid-mechanical quantities simply by changing the time sequence or light-sheet position. For constant pulse separation ( $\Delta t=t_{2}-t_{1}=t_{3}-t_{2}=t_{4}-t_{3}$ ) and overlapping light-sheets, a time sequence of three velocity fields can be measured at any repetition rate by cross-correlating the first acquired grey-level distribution with the second, the second with the third and the third with the grey-level distribution from the last illumination, see figure 4.5. By increasing the time delay between the second and third illumination $\left(\Delta t=t_{2} \overline{-t_{1}=t_{4}}-t_{3}<t_{3}-t_{2}\right)$ the first order estimation of the acceleration field in its Lagrangian and Eulerian form can be calculated in order to study the dynamic behaviour and the interaction processes of moving flow structures [36]. The Lagrangian acceleration of a moving fluid-element is defined as the temporal derivative of the velocity and can be simply implemented as a difference quotient

$$
a_{i, j}(t)=\frac{U_{i, j}\left(t^{\prime \prime}\right)-U_{i, j}\left(t^{\prime}\right)}{t^{\prime \prime}-t^{\prime}}
$$

whereby $t^{\prime}$ and $t^{\prime \prime}$ are defined as the mean between the corresponding illuminations e.g. $t^{\prime}=$ $\left(t_{2}+t_{1}\right) / 2$ and $t^{\prime \prime}=\left(t_{4}+t_{3}\right) / 2$ respectively and $u_{i, j}$ denote the velocity of a fluid volume at any time $t$ (the indices $i, j$ denote the discrete location in the measurement plane). Equation (4.1) states that in case that $u_{i, j}(t)$ is continuous in $t^{\prime} \leq t \leq t^{\prime \prime}$ and differentiable in $t^{\prime}<t<t^{\prime \prime}$ there exists a $t$ in the specified time-interval for which the acceleration is given by expression 4.1. The exact time $t$ is unknown except for the case of a linear variation of the velocity during the time interval. In case of the Eulerian acceleration

$$
\underline{a}(\underline{x}, t)=\frac{\partial \underline{U}(\underline{x}, t)}{\partial t}+\underline{U}(\underline{x}, t) \cdot \nabla \underline{U}(\underline{x}, t)
$$

the first term on the right hand side can be approximated by

$$
\frac{\partial \underline{U}(\underline{x}, t)}{\partial t}=\frac{\underline{U}\left(\underline{x}, t^{\prime \prime}\right)-\underline{U}\left(\underline{x}, t^{\prime}\right)}{t^{\prime \prime}-t^{\prime}}
$$

with the fluid velocities $\underline{U}\left(\underline{x}, t^{\prime}\right)$ and $\underline{U}\left(\underline{x}, t^{\prime \prime}\right)$ measured at $\underline{x}$ and at times $t^{\prime}$ and $t^{\prime \prime}$ respectively. The nonlinear term on the right hand side of equation 4.2 can be approximated to: 
FIGURE 4.5: Timing diagram for the temporally separated determination of all three velocity components. Different shading of the light-sheet profile indicates different states of polarisation.

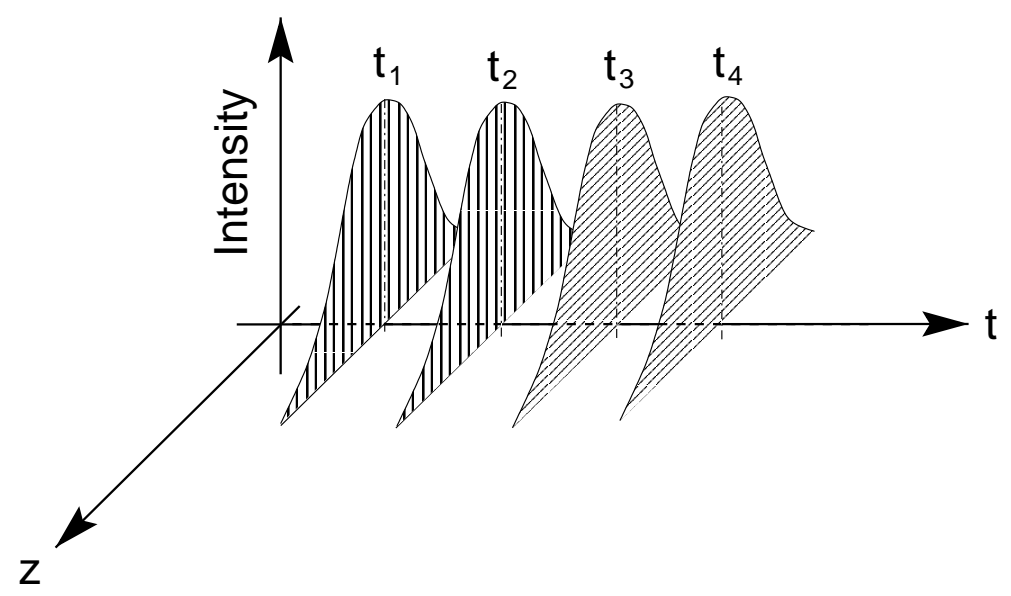

$$
\underline{U}(\underline{x}, t) \cdot \nabla \underline{U}(\underline{x}, t)=\frac{1}{2} \sum_{i, j}\left[\frac{\partial U_{i}\left(\underline{x}, t^{\prime}\right)}{\partial x_{j}} u_{j}\left(\underline{x}, t^{\prime}\right)+\frac{\partial U_{i}\left(\underline{x}, t^{\prime \prime}\right)}{\partial x_{j}} U_{j}\left(\underline{x}, t^{\prime \prime}\right)\right] \underline{e}_{i}
$$

where $\underline{e}_{i}$ with $(i=1,2,3)$ indicates the three unit vectors. By fitting each component of the spatial velocity gradient at a given point to its two adjacent velocity vectors along the axis of the given component the partial velocity gradients can be determined from a pair of velocity fields. The two products containing the partial velocity gradients are determined from each of the velocity fields and are averaged together, for details see [36].

Besides of measuring the acceleration the temporal behaviour of the moving flow structures can be estimated as well which yields information about the formation and decay of flow structures. Furthermore, three dimensional space-time correlations can be measured simply by increasing the time delay between a pair of images being acquired $\left(\Delta t=t_{2}-t_{1}=t_{4}-t_{3} \ll\right.$ $\left.t_{3}-t_{2}\right)$ with $\overline{U_{i}}(x, y)=\sum_{i=1}^{n} U_{i}(x, y, t) / n$.

$$
R_{u_{i} u_{j}}(\Delta x, \Delta y, \Delta t)=\frac{\sum_{i=1}^{m} \sum_{j=1}^{n}\left[U_{i}-\overline{U_{i}}\right]\left[U_{j}-\overline{U_{j}}\right]}{\sqrt{\sum_{i=1}^{m} \sum_{j=1}^{n}\left[U_{i}-\overline{U_{i}}\right]^{2} \sum_{i=1}^{m} \sum_{j=1}^{n}\left[U_{i}-\overline{U_{i}}\right]^{2}}}
$$

When the light-sheet pairs with equal polarisation are spatially separated as indicated in figure 4.6 further important information about the flow field can be achieved. For small separations (or partially overlapping light-sheets) the multiplane stereo PIV technique allows the determination of all three components of the vorticity vector - PIV and Stereo-PIV offer only one component - or the divergence of the velocity field, according to equations 4.4 and 4.5.

$$
\begin{aligned}
\operatorname{rot} \underline{U}=\nabla \times \underline{U}= & {\left[\frac{\partial W}{\partial y}-\frac{\partial V}{\partial z}\right] \underline{e}_{x}+\left[\frac{\partial U}{\partial z}-\frac{\partial W}{\partial x}\right] \underline{e}_{y}+\left[\frac{\partial V}{\partial x}-\frac{\partial U}{\partial y}\right] \underline{e}_{z} } \\
& \operatorname{div} \underline{U}=\nabla \cdot \underline{U}=\frac{\partial U}{\partial x}+\frac{\partial V}{\partial y}+\frac{\partial W}{\partial z}
\end{aligned}
$$

This requires that the differently polarised light-pulses are fired simultaneously $\left(t_{1}=t_{3}\right.$ and $t_{2}=t_{4}$ ) as shown in figure 4.6.

The same data can be analysed in terms of critical point theory which displays mathematically the important features of a given set of first order differential equations without having 


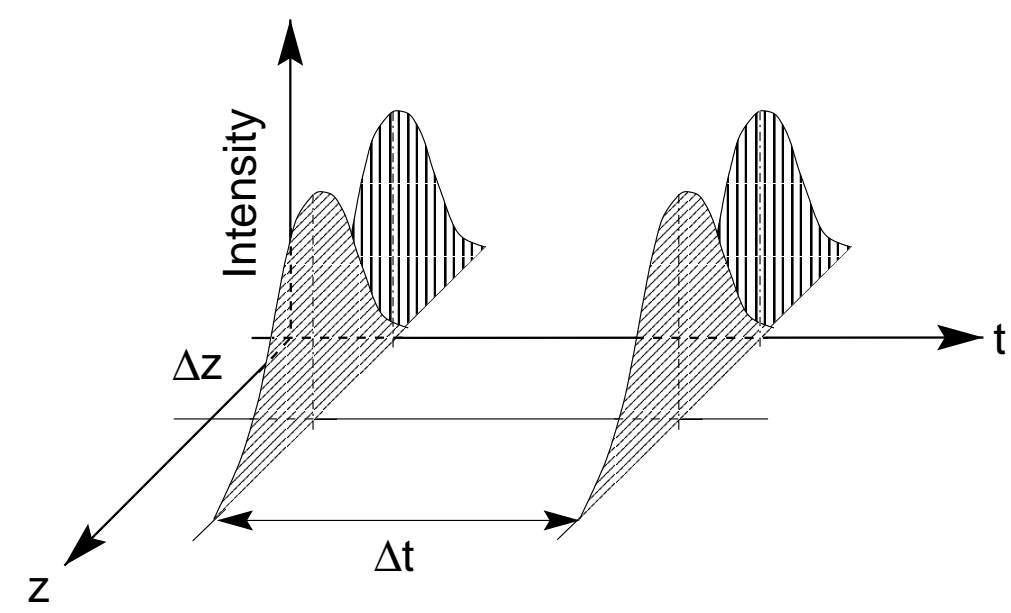

FIGURE 4.6: Timing diagram for the simultaneous determination of all three velocity components in spatially separated planes

the exact solutions. This analysis, which allows vortex identification to be made more reliable, involves locating certain critical points, linearising the equation in its vicinity and examining the topological features of the solution trajectories. This leads to the classification of possible critical points which may be displayed in a (P,Q,R)-diagram [82].

For calculating gradients normal to the light-sheets (i.e. $\partial U_{i} / \partial z$ with $i=1,2,3$ ) it is obvious that either the forward- or backward-difference scheme has to be applied as other extrapolation techniques require three or more grid points in each spatial direction. This is not a disadvantage (especially in terms of spatial resolution) as long as the spacing between the light-sheets is sufficiently large so that the data entering in the formula is not correlated. For the in-plane derivatives (e.g. $\partial U_{i} / \partial x$ and $\partial U_{i} / \partial y$ with $i=1,2,3$ ) the three or even five point difference scheme is more suited especially for 50 percent interrogation window overlap analysis in order to reduce the truncation or random errors in the values of the velocity function [109].

By increasing the spacing $\Delta z$ between the light-sheet pairs and varying the time delay between the second and third illumination, all components of the four dimensional spatiotemporal correlation tensor $R_{u_{i} u_{j}}$ can be measured according to

$$
R_{u_{i} u_{j}}(\Delta x, \Delta y, \Delta z, \Delta t)=\frac{\sum_{i=1}^{m} \sum_{j=1}^{n}\left[U_{i}-\overline{U_{i}}\right]\left[U_{j}-\overline{U_{j}}\right]}{\sqrt{\sum_{i=1}^{m} \sum_{j=1}^{n}\left[U_{i}-\overline{U_{i}}\right]^{2} \sum_{i=1}^{m} \sum_{j=1}^{n}\left[U_{i}-\overline{U_{i}}\right]^{2}}}
$$

Furthermore the direction of a vortex which crosses these planes can be determined for $\Delta z \neq 0$ and $\Delta t=0$ (this is especially valuable for aircraft wake vortex investigations) and many other quantities not mentioned here which are helpful for the understanding of fluid-mechanical problems, see [46, 49].

\subsection{Modes of Operation II - Out-of-plane flows}

Three dimensional flows with a wide range of different time and length scales always cause problems because the time delay between the two illuminations has to be reduced in order to keep the out-of-plane loss-of-pairs at an acceptable level. As this results in a short in-plane displacement between corresponding particle image pairs (often less than one pixel) accompanied 
by a vanishing (or not resolvable) displacement variation, peak-looking effects (introduced by the under-sampled particle images and amplified due to the peak-fit for sub-pixel accuracy in the correlation plane [105]) become dominant and may lead to misinterpretation of the data. In order to avoid any artificial software based solutions which require a priory knowledge and are not based on physical grounds, the magnification of the imaging system needs to be changed such that small variations in the object plane yield resolvable variations in the image plane. Using the multiplane technique, this problem can be solved without reducing the field of view by rearranging the linearly orthogonally polarised light-sheets in an appropriate way as shown in figure 4.7. In the case of a strongly varying out-of-plane component in only one direction (main flow perpendicular to the light-sheet along $z$ ), all four light-sheets should be separated (not necessarily equally spaced) as shown in figure 4.7. This can be easily performed by rotating mirrors $8 \mathrm{a}$ and $8 \mathrm{~b}$ in figure 4.2 or 4.3 slightly in opposite directions and mirror $8 \mathrm{c}$ in one direction.

FIGURE 4.7: Optimised lightsheet positioning to reduce lossof-pairs induced by out-of-plane motion for a cross-flow in only one direction. Different shading of the light-sheet profile indicates different states of polarisation.

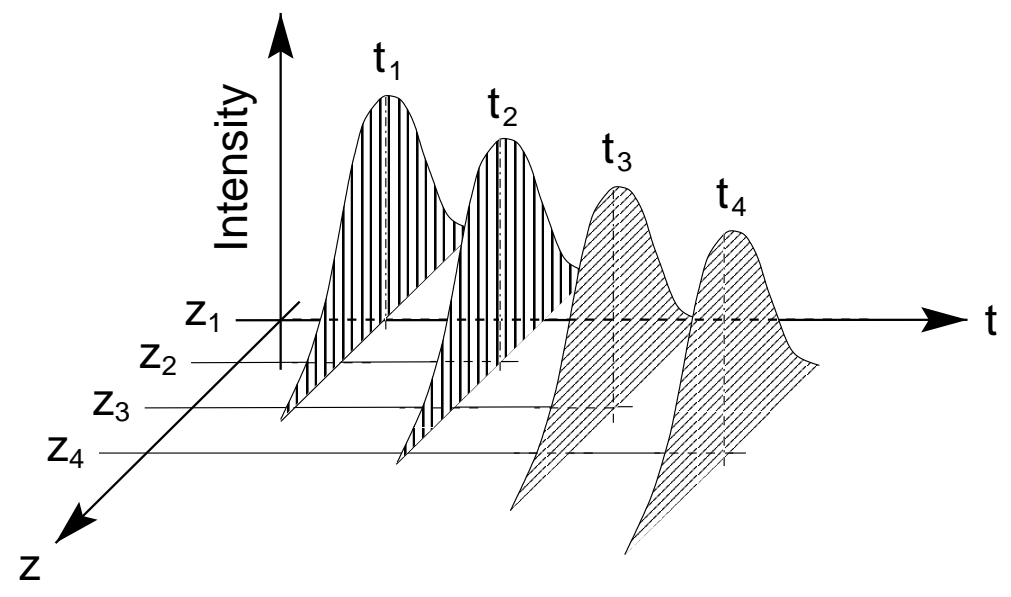

The advantage of this light-sheet positioning lies in the ability to determine three different time scales or three different length scales nearly independently for a given time delay or pulse sequence. The result is a set of three different displacement fields - by correlating the first acquired grey-level distribution of a sequence with each of the following single exposed fields - from which one optimised displacement field can be calculated by comparing the correlation coefficients for each location $(x, y)$ in all three displacement fields. This technique can be seen as an extension of the well known multi-pass interrogation technique into the third dimension. For example let all particles selected during the first exposure at $t_{1}$ move in positive $z$-direction during the time interval $t_{i}-t_{1}(\mathrm{i}=2,3,4)$. When $t_{2}=t_{3} \simeq t_{4}\left(t_{3}=t_{4}\right.$ is impossible as both light-sheets possess the same state of polarisation) a correlation between the grey level distribution from the 1st exposure with the others gives a high correlation coefficient in regions where the particle displacement matches the light-sheet displacement. In the case of $t_{1}=t_{3}$ and $t_{2}=t_{4}$, on the other hand, the accuracy of vorticity and acceleration measurements can be significantly increased for out-of-plane flows and the case $t_{1}<t_{2}<t_{3}<t_{4}$ may help to reduce the previously mentioned peak-locking effect. Time correlations can be performed with the same light-sheet configuration or alternatively using the arrangement in figure 4.5 along with the Taylor hypothesis. 


\subsection{Simplified recording system}

For some experiments it might be sufficient to replace the complex recording unit outlined in figure 4.1 by a simple stereoscopic PIV system. When the first two illuminations are stored onto the first fields of the progressive scan CCD camera pair in stereoscopic arrangement and the second two illuminations onto the last field of both cameras, each of the four double exposed images can be analysed using the conventional evaluation method based on the autocorrelation technique [109]. By combining the corresponding displacement fields, two independent three-component displacement fields can be obtained. The time separation between these displacement fields can be chosen arbitrarily depending on the quantity in question (acceleration, space-time correlation or proper orthogonal decomposition are relevant values for example). For constant pulse separation and overlapping light-sheets, a third displacement field can be calculated as well by cross-correlating the two double exposed images from each camera. For overlapping light-sheet arrangements there are almost no drawbacks with respect to the polarising beam-splitter based system except the reduced spatial resolution due to the evaluation technique and the direction ambiguity [51]. In the case of spatially separated light-sheet pairs there are two drawbacks. Firstly, the simultaneous measurement in time is excluded, so the vorticity and the velocity gradient tensor based values cannot be determined. Secondly, the spatial separation between the light-sheets is limited by the depth-of-focus of the imaging system as the same lens has to be used for the measurement in both planes. The latter restriction can be influenced by the aperture and focal length of the imaging system. Although the power of this four-pulse technique is limited with respect to the previously described arrangement, it provides the user with much more fluid-mechanical information compared to a conventional two pulse stereoscopic PIV system.

\subsection{Polarisation effects}

When the Multiplane Stereo PIV technique is applied it is important to keep various optical effects into account in order to maximise the performance of the measurement technique. The first two undesired effects the experimentalist has to deal with results from the multiple reflections between optical components, because they reduce the contrast in the captured image and the light scattered in the object direction is lost. To avoid these effects, the optical elements are coated with a thin layer of material that reflections from the outer surface of the film and the outer surface of the substrate cancel each other by destructive interference or interfere constructively in order to produce a highly efficient reflector. The requirements to create an exact cancellation of the reflected beams with a single layer coating are that the path difference of the reflections is exactly an integral number of half wavelength $\left(180^{\circ}\right.$ out of phase) and of the same intensity. The first requirement is automatically fulfilled as during external reflection the light waves undergo a $180^{\circ}$ phase shift (no such phase shift occurs for internal reflection except in the case of total internal reflection). To fulfil the last requirement, the coating material must be chosen in such a way that the refractive index $n_{\text {air }}$ and $n_{\text {film }}$ are equal for a given wavelength $n_{\text {air }} / n_{\text {film }}=n_{\text {film }} / n_{\text {substrate }}$. For $n_{\text {air }}=1$ follows $n_{\text {film }}=\sqrt{n_{\text {substrate }}}$. Assuming the last condition cannot be fulfilled by any known material or the reflectance by a single layer is not sufficient enough for the application, a multi-layer coating is necessary. V-coatings are alternate high and low refractive index films, each one having an optical thickness of $1 / 4$ of a wave (at the design wavelength), which reduce the reflection of a component to near-zero. 
The disadvantage of these films is their extreme sensitivity to both wavelength and angle of incidence (the effect of increasing the angle of incidence equals to a shift to slightly shorter wavelength and an increase of the long wavelength reflectance). Since multiple-plane stereo PIV applications involve other than normal angles of incidence and high numerical aperture (low f-number) optics, it is better to use a broadband anti-reflection coating. These are multilayer films, comprising alternate layers of various index materials, which reduce the overall reflectance to an extremely low level for the broad spectral range covered.

Polarisation effects are normally not considered for anti-reflection coatings as these are nearly always used at normal incidence where the two polarisation components are equivalent. High reflectance or partially reflecting coatings, which work on the same principles as dielectric anti-reflection coatings, are frequently used away from normal incidence, particularly at $45^{\circ}$ for mirroring or beam-splitting purposes and the polarisation plane is arbitrarily orientated with respect to the plane of incidence. Under these conditions the maximum spolarisation reflectance is always greater than the maximum p-polarisation reflectance due to the difference in effective refractive index of the coating for the s- and p-components of the incident beam as can be seen from Fresnel equations or figure 4.8. Thus, the reflected (or
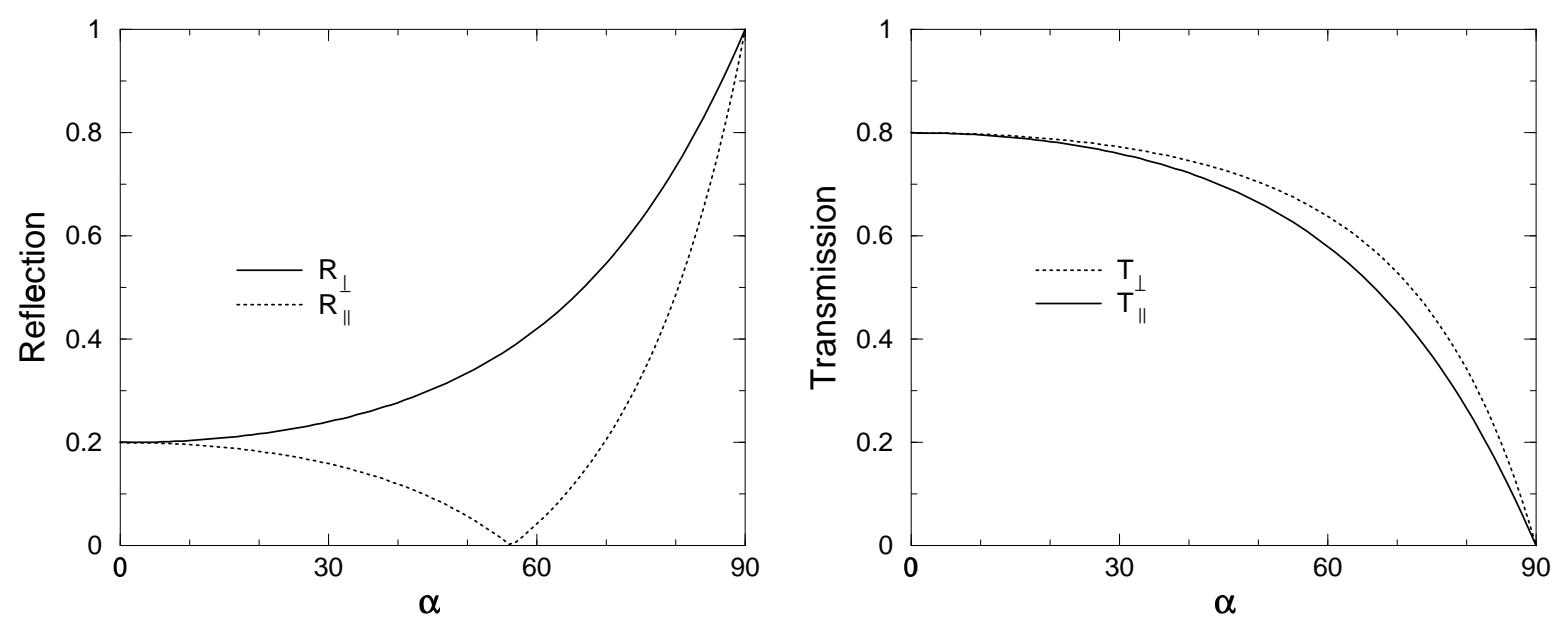

FIGURE 4.8: Reflectance and transmission of a plane monochromatic wave with $\lambda=532 \mathrm{~nm}$ at a planar glass interface as a function of the angle of incidence $\alpha$ and state of polarisation.

transmitted) beam is still linearly polarised, but the oscillation plane of the electric field vector has rotated relative to the plane of incidence as the magnitude of both reflected components is different. By observing the illuminated flow under a certain angle of incidence through the polarising beam-splitter cube, the intensity variation over the field of view (increasing from the centre to the edge) can be easily observed for appropriate choice of polarised light and beam-splitter orientation. These effects have to be taken into account before arranging the multiple-plane stereo PIV, especially for the large field of view applications.

Since the polarisation of the scattered light, as a function of the observation angle, mainly depends on the diameter of the particles (for spheres of finite conductivity and finite dielectric constant), the separation of the light using a polarising beam-splitter cube might not be accurate enough when the variance of the particle diameter is large, and the spacing between the light-sheets is of the order of the depth-of-focus (for larger distances the weakly depolarised particles are out of focus and thus far below the digital registration threshold). Weakly 
reflected ghost-images superimposed on the desired image result in a reduced signal-to-noise ratio accompanied by a lower correlation coefficient. To solve this problem, the time separation method can be applied as long as the separation between the first orthogonal polarised light pulse pair is negligible, e.g. the position of the particle image remains constant in terms of digital registration. In this case, which holds for a wide range of flow velocities, a difference between instantaneous and time separated measurements cannot be observed. Technologically, this can be done by transferring the first image right behind the first illumination.

\subsection{Monochromatic aberrations}

Before the light scattered by the particles can be recorded it interacts partially with optical elements like glass-window, mirror, beam-splitter and various lenses in order to alter the orientation of the field, to separate the incoming light according to the state of polarisation and to generate an image of the tracer particles. Beside a possible loss of light due to absorption or reflection, these components introduce aberrations of different type, direction and magnitude which may limit the measurement accuracy [8]. Although it is impossible to eliminate all aberrations completely in any real system of finite aperture, a basic understanding of their origin and dependence from optical parameters is the key to reduce these undesirable effects under the resolution of the recording medium or to eliminate certain aberrations completely by accepting aberrations of other types which are of no harm in PIV. The last point is of primary importance because higher order aberrations like distortion ${ }^{1}$ and curvature of the field just influence the position and form of the image but do not lower the resolution. They can be completely eliminated by calculations according to section 2.4 and do not need to be considered here. Primary aberrations on the other hand like spherical aberration, coma and especially astigmatism deteriorate the image and alter the shape in a characteristic way. This leads to an increased measurement error as the performance of the peak-fit for sub-pixel accuracy strongly decreases for particle image diameter not equal 2-3 pixel. Before the optical aberrations can be reduced or eliminated, they have to be identified first. Using the PIV equipment this is easily possible as higher order aberrations become clearly visible by analysing the image of a regular grid, according to section 3.2, whereas the main primary aberrations can be easily observed by examination the image-symmetry of small particles within a thin light-sheet. For three different field-coordinates figure 4.9 shows the images of olive-oil droplets, with $d_{p}=1 \mu \mathrm{m}$, through a tilted BK7 glass-plate of constant thickness $(10 \mathrm{~mm})$. The most striking feature is the variation of the magnitude and direction of the dominant aberration by probing continually through focus and across the field of view.

When the optical system is perfectly aligned and the aberrations are below digital registration, the diffraction limited image of a particle appears as a bright circular core surrounded by several rings of rapidly diminishing brightness. This is shown in the central column of figure 4.9 but the small variations of the intensity distribution are smeared out by the low resolution of the used CCD sensor. As the object moves further off-axis, the diffraction limited particle image pattern alters from a bright central area surrounded by dark and bright rings to a dark central area surrounded by bright and dark rings as shown in the lower image. On the other hand, particles, located an appreciable distance apart from the optical axis deform into a line of certain orientation due to the tilted glass-window, see outer image pair of top row. By

\footnotetext{
${ }^{1}$ Distortion occurs when off-axis points are not formed at the location predicted by the paraxial equations.
} 

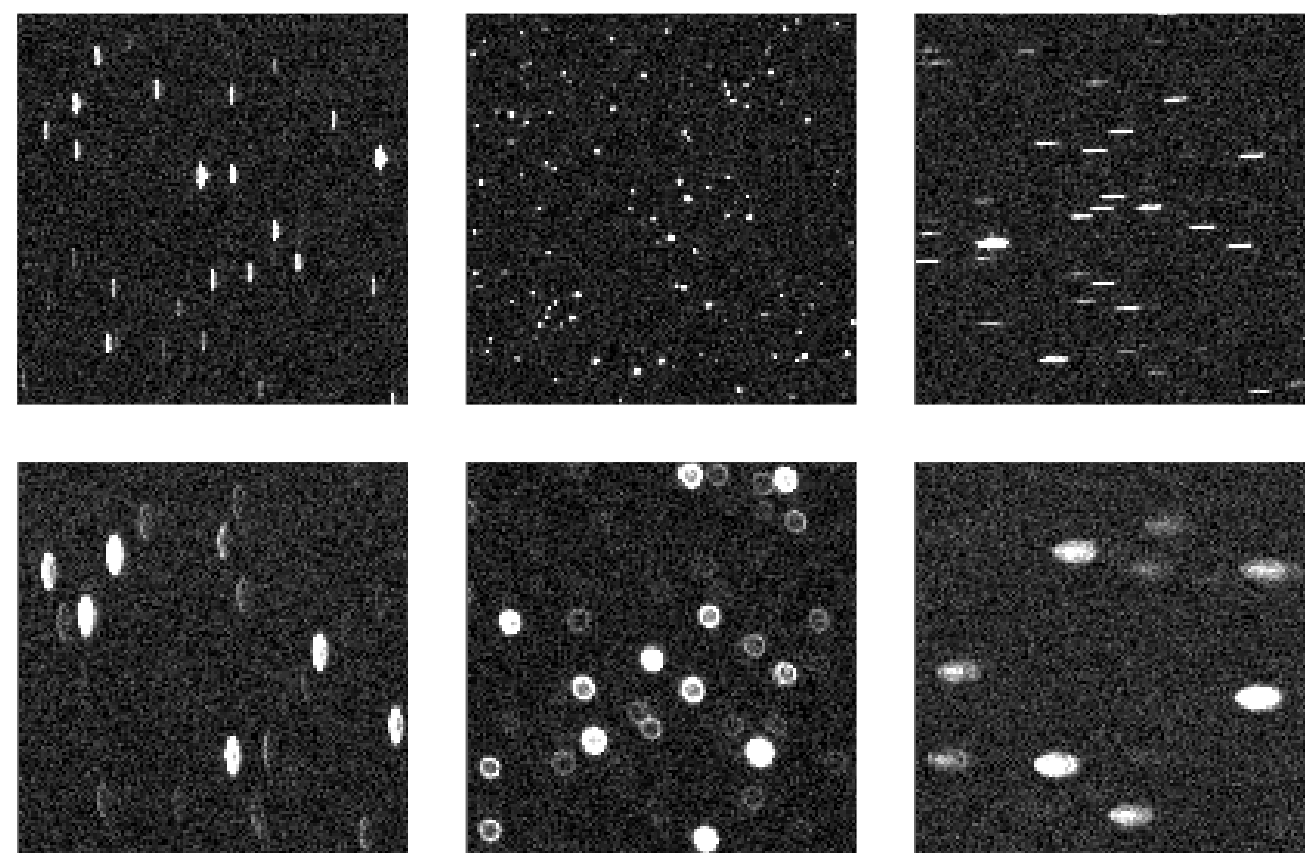

FIGURE 4.9: Images of tracer particles $\left(d_{p}=1 \mu \mathrm{m}\right)$ observed through a tilted glass-plate (BK7) of constant thickness $10 \mathrm{~mm}$ for three field locations as a function of the focus. The lines of constant intensity (isophote) are circular near the centre of the field but have a more complex form in the outer part of the image. The size of each sample is 128 pixel $^{2}$.

changing the focus this line becomes elliptical and out-of-focus effects start to occur like the decreased brightness and the intensity gap in the centre of the particle-image, compare outer image pairs of figure 4.9.

The mentioned deformation is the main aberration the experimentalist has to deal with in praxis. It appears already when a non-collimated light-front enters or passes planar optical elements of different index of refraction, such as glass windows which separate the test-section from the laboratory, and it becomes even more pronounced for curved interfaces like lenses [6]. Although a detailed analysis of aberrations requires the theory of diffraction in order to account for the intensity distribution, the main features become evident by using the principles of geometrical optics which identify the image by the points of intersection of the geometrical rays with the image plane. Using this approximation, the starting point of this consideration is the law of refraction $n_{1} \sin \theta_{1}=n_{2} \sin \theta_{2}$ which describes the direction of a light ray after diffraction at the planar interface between two homogeneous, isotropic media of differing index of refraction. To obtain the image position $z$ of an object located on the optical axis at $Z$, this law has to be applied for each ray emerging from this point. Using the relations $\theta_{1}=-\alpha_{1}$ and $\theta_{2}=-\alpha_{2}$ according to figure 4.10 , the following formula can be derived.

$$
z=\frac{n_{2} Z}{n_{1} \cos \alpha_{1}} \sqrt{1-\left(\frac{n_{1}}{n_{2}} \sin \alpha_{1}\right)^{2}}
$$

This equation implies the appearance of aberration as the geometrical picture of an objectpoint does not possess a unique image point. For each aperture-angle $\alpha_{1}$ there exists a certain 


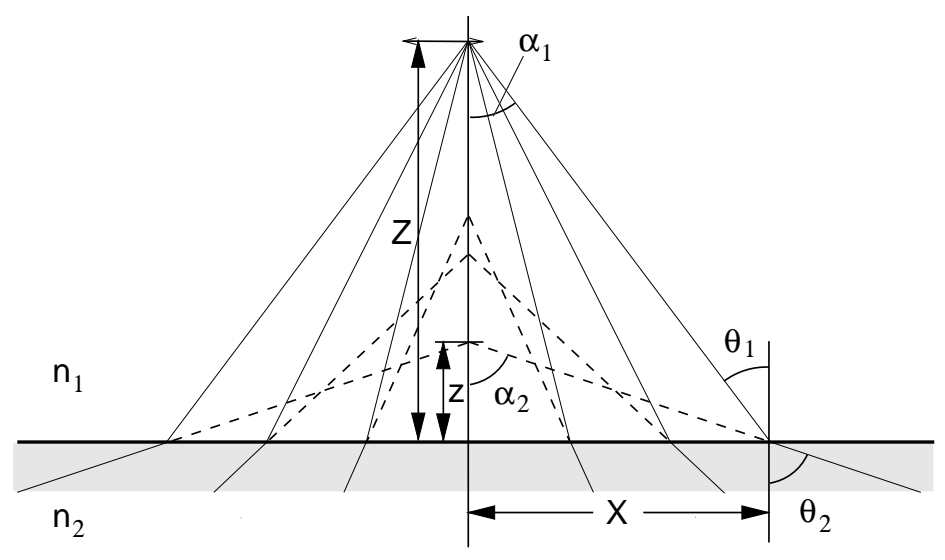

FIGURE 4.10: Imaging due to refraction at a plane surface at finite aperture after [6].

intersection-width $z$ whose exact location depends on the object-distance $Z$ and the index of refraction of the two media. In addition, the magnitude of the aberrations is proportional to the angle of incidence, because the variation of the intersection width $\Delta z$ increases with increasing aperture angle. The limit $Z \rightarrow \infty$ is important as all rays intersect in only one point located at infinity and no aberrations occur at all. This situation can be generated either by using an optical collimator which forms parallel rays or by stopping the lens in a way that all rays are nearly parallel before they enter the planar interface. The last possibility is frequently used in PIV but its applicability mainly depends on the output energy of the laser, on the cross-section of the light-sheet, on the scattering behaviour of the particles and finally on the sensitivity of the CCD camera. It should be emphasised that in contrast to the refraction, reflections at a planar interface are aberration free, because all light rays from an object perfectly intersect in one virtual image point independent of the angle of incidence on the mirror. This is different for non planar surfaces where caustics can be observed.

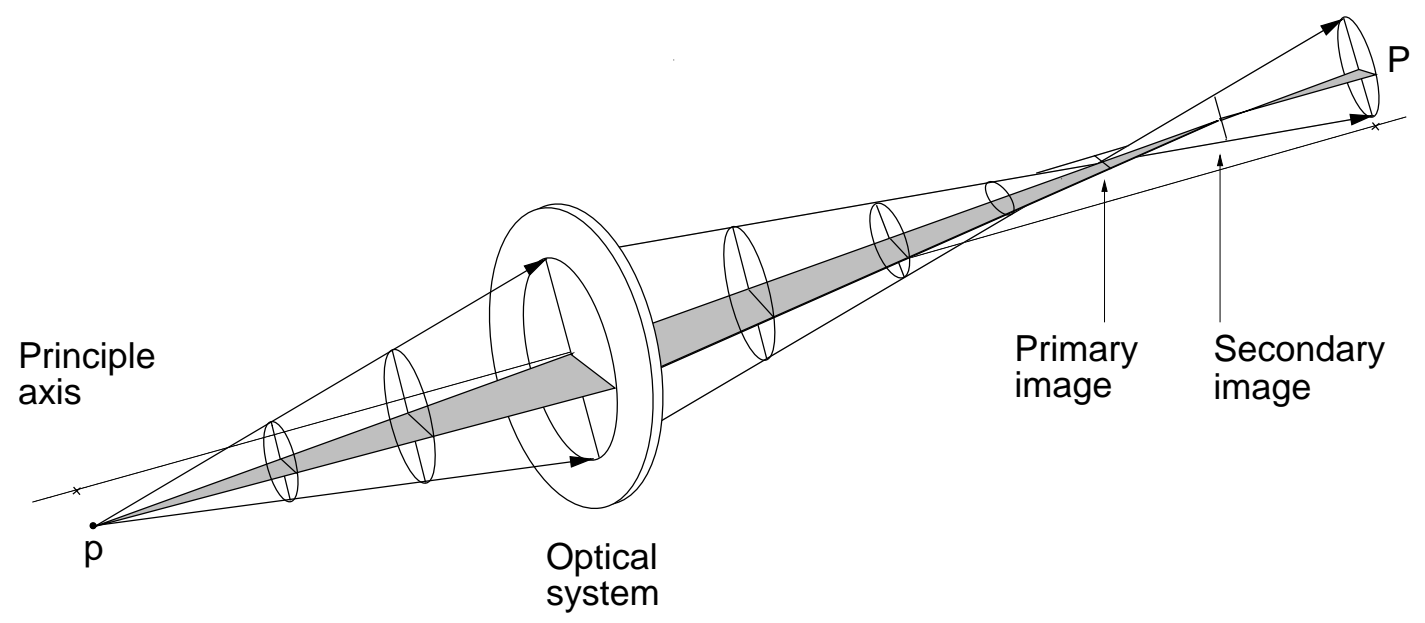

FIGURE 4.11: Schematic representation of the image size and orientation for a non-axial object point as a function of the lateral position after [6].

When the situation is considered where an object point lies a considerable distance away from the optical axis, as indicated in figure 4.11, the incident cone of rays will strike the lens in an asymmetrical way. As a result, the focal length in this plane will be different as well due to the different optical path. In effect the meridional rays are tilted more with 


\section{Multiplane Stereo Particle Image Velocimetry}

regard to the lens than the sagittal rays and they have a shorter focal length. This results in a significant change in symmetry as a function of the image distance. The initially circular cross section of the beam, emerging from the lens, becomes elliptically with the major axis in the sagittal plane and degenerates into a line (primary image) at the tangential or meridional focus. Beyond this point the beam cross-section rapidly expands until it is again circular (circle of least confusion). Moving further away from the lens the beam cross-section again deforms into a line (secondary image) while the orientation of this line is rotated by $90^{\circ}$ relative to the primary image. This behaviour is similar to that shown in figure 4.9 and can be studied best by probing throughout the focus while observing non-axial particle images.

Aberrations introduced by means of the optical components within the laser, as the frequency doubler crystal or for the generation of the light-sheet, are more difficult to observe but of similar importance for PIV. Both systems have to be carefully aligned in order to obtain the best possible signal in the image-plane as information loss in this stage cannot be reconstructed, especially not by software solutions. The first testing procedure on any lens, after it
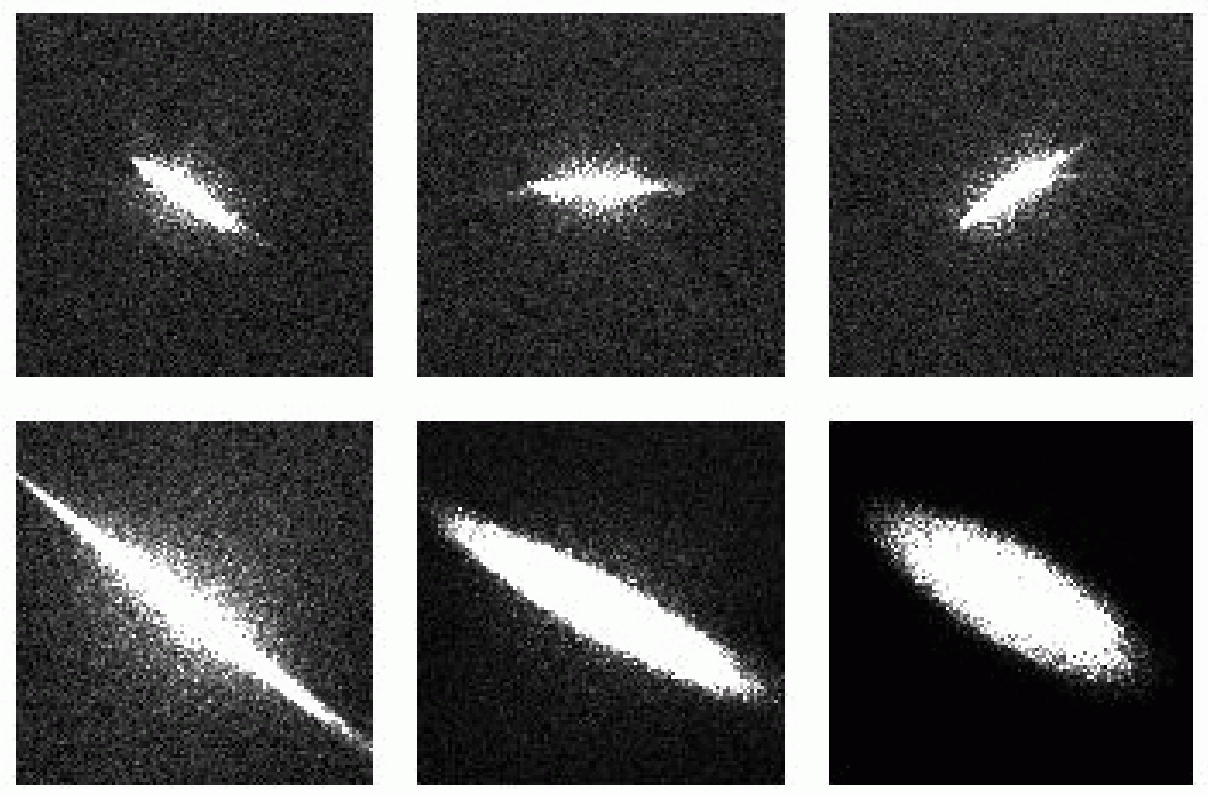

FIGURE 4.12: Top row: Intensity distribution of a Nd:YAG beam behind a tilted converging lens for three different angles of rotation. Bottom row: dependence of the light-sheet profile on the magnitude and direction of the aberration.

has been set up in the light-sheet-optics, is to rotate the optical elements about their own axis while examining the image. If there should be any de-centreing or tilt, lateral asymmetries in the point image will appear to rotate with the lens. This is shown in the top row of figure 4.12 for three different angles of rotation. The bottom row of the same figure reveals the same operation, but a fixed cylindrical lens was placed behind the rotating lens in order to highlight the direct dependence of the light-sheet profile from the magnitude and direction of the aberration. Generating an extremely thin light-sheet, which is necessary for high resolution 
experiments in turbulent boundary layers for instance, requires that either no aberrations are present or the major axis of the aberration coincides with the respective axis of the cylindrical lens. Otherwise the performance of the light-sheet is biased by the aberration.

\subsection{Feasibility study}

To study the accuracy of the light separation and the reliability of the multiplane stereo system described in the previous sections, the acoustic receptivity of a laminar boundary layer along a flat plate with zero pressure gradient was examined. The measurement was performed in the open circuit low turbulence wind tunnel at DLR, which possesses a contraction ratio of $15: 1$ and a cross section of $0.3 \times 1.5 \mathrm{~m}^{2}$, see figure 4.13 . To obtain reproducible conditions
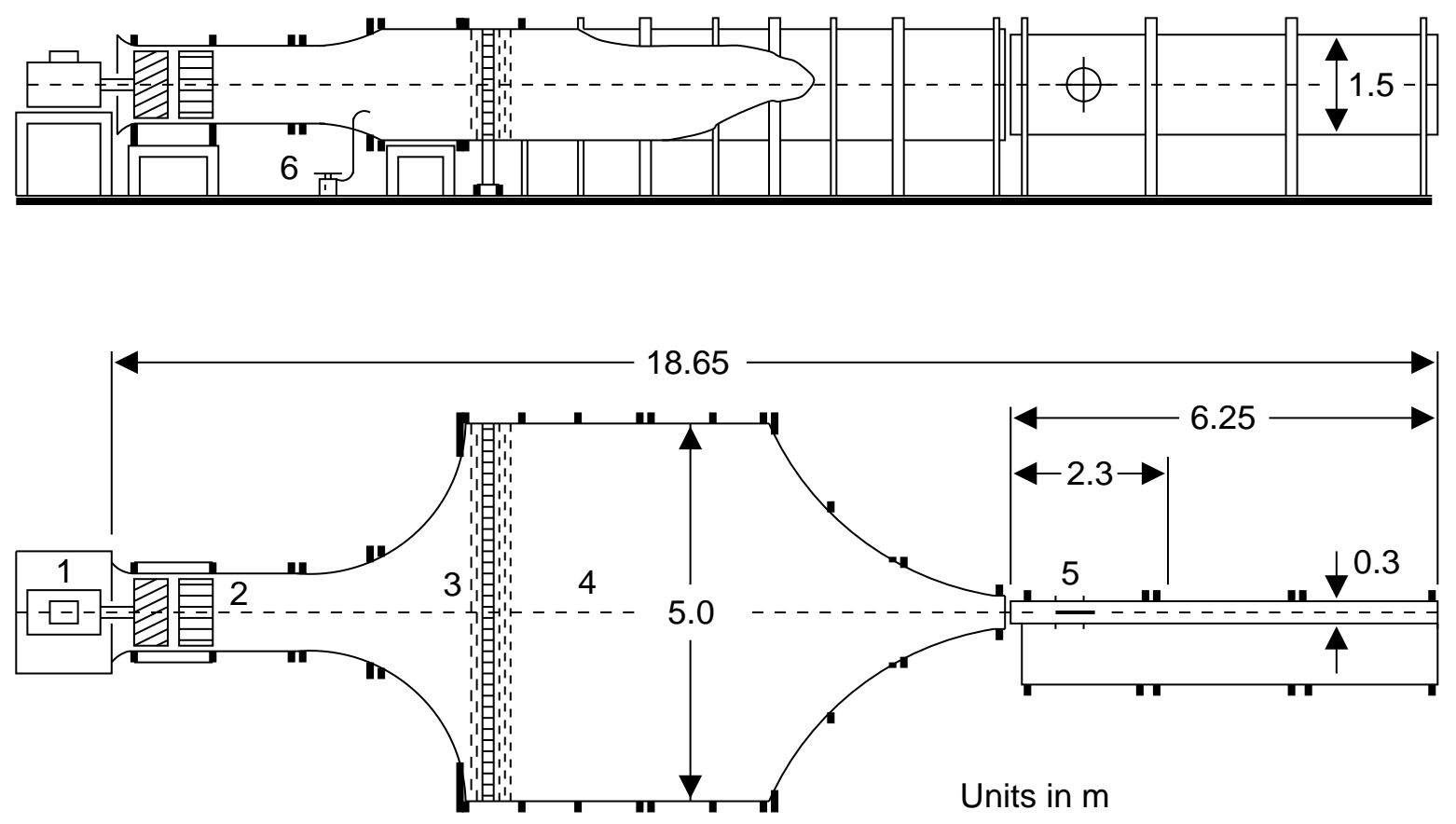

FIGURE 4.13: Low turbulence wind-tunnel at DLR Göttingen (TUG). Top: Side view. Bottom: Top view. 1 engine, 2 fan, 3 honeycomb and screens, 4 settling chamber, 5 side window, 6 seeding generator, 7 recording system.

for the generation and development of small disturbances, an acoustic excitation device was applied in the form of a span-wise slot pair, $400 \mathrm{~mm}$ in length and $0.3 \mathrm{~mm}$ in width, which was connected to an amplifier loudspeaker system in such a way that span-wise and oblique waves could be generated simultaneously, for details see [107]. By changing the amplitude of the disturbance, different stages of $\Lambda$-vortices could be generated for a given free-stream velocity $(12 \mathrm{~m} / \mathrm{s})$ and frequency of the acoustic excitation $(150 \mathrm{~Hz})$, see figure 4.14 . The slot pair was located $200 \mathrm{~mm}$ behind the elliptically shaped leading edge of the smooth plate and embedded with high accuracy to minimise uncontrollable disturbances of the flow. The dimension of the plate in stream-wise and span-wise direction are $1175 \mathrm{~mm} \times 1500 \mathrm{~mm}$ to avoid side effects introduced by the walls of the wind tunnel test-section.

To generate a homogeneous seeding and to avoid artificial flow disturbances due to the injection of the tracer particles, the whole laboratory was seeded before starting the experi- 


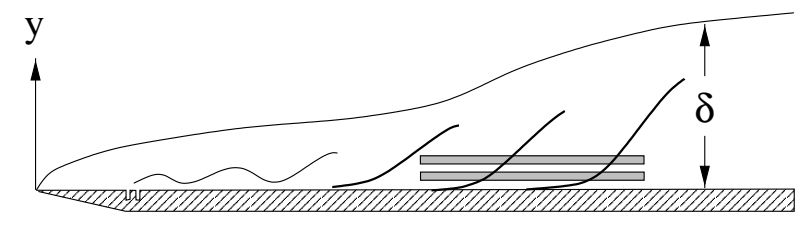

FIGURE 4.14: Development of $\Lambda$-vortices along a flat plate (side and top view). The rectangles represent the light-sheet position and field of view.

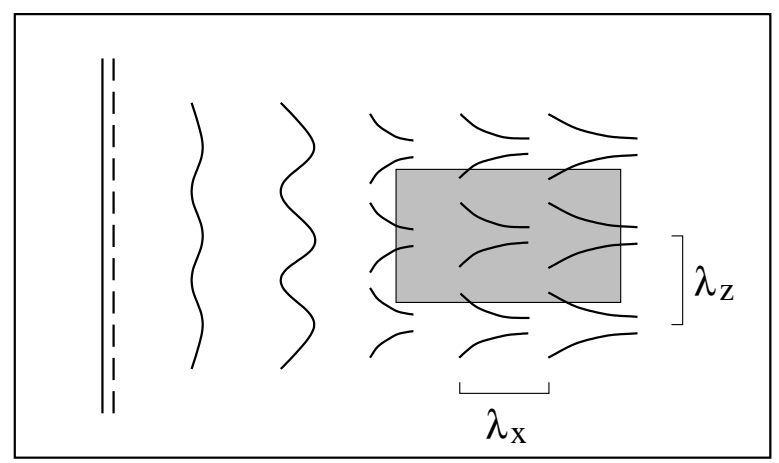

ment. For the illumination of the $1 \mu \mathrm{m}$ olive oil droplets, generated by means of multi-hole nozzles described in section 2.2, a four pulse Nd:YAG laser-system (Quantel Brillant B) in the configuration shown in figure 4.2 with an output energy of $320 \mathrm{~mJ}$ per pulse at $\lambda=532 \mathrm{~nm}$ was used. Due to the small boundary layer thickness at the beginning of the measurement position $(\delta=5 \mathrm{~mm}$ ) a combination of four lenses (focal length in $\mathrm{mm}$ : -150 (bi-concave), 200 (bi-convex), 60 and 100 (both planar convex)) was necessary to form a sufficiently thin lightsheet $(0.5 \mathrm{~mm})$ nearly constant in thickness for over more than $1 \mathrm{~m}$. For the recording of the particle images four Peltier cooled PCO cameras were used with $1280 \times 1024$ pixel resolution and 12 Bit dynamic range at $12.5 \mathrm{MHz}$ readout frequency. The read-out noise of the PCI interface board is less than 8 electrons. The pixel size is $6.7 \mu \mathrm{m}^{2}$ and the dimension of the chip is $2 / 3^{\prime \prime}$. For magnification and field of view adjustments (necessary for maximising the amount of stereo information) all Scheimpflug-adapters were mounted on a two-axis linear translation stage which allows high precision translations by thumb screws. The opening angle between the cameras was $\alpha=40^{\circ}$ and the magnification factor on the optical axis $\mathrm{M}=0.1$ for all cameras. For the imaging four $60 \mathrm{~mm}$ macro planar lenses (Carl Zeiss) were used with an aperture of 8 to avoid optical aberrations introduced by the optical components in a non-collimated beam according to section 4.7. After the beam-splitter and the $\lambda / 2$ plates had been properly aligned, polarisation changes during the scattering process (at very large particles or particle clusters) or the diffraction at the test-section window as outlined in section 4.6 could not be observed under these conditions. Usually the intensity of ghost-images due to polarisation changes during the scattering process is far below the minimum sensitivity level of the CCD sensor (for small particles). However, when polarisation effects occur time separation can be applied as well, as long as the separation between the first orthogonally polarised light pulse pair is negligible, e.g. the position of the particle image remains constant in terms of digital registration. In this case, which holds for a wide range of flow velocities, a difference between instantaneous and time separated measurements cannot be observed. Technologically, this can be done by transferring the first acquired images right behind the first illumination so that the second illumination with differently polarised light cannot be recorded by means of the previous system.

To trigger all necessary components quickly and easily, a 16 channel sequencer-board with 

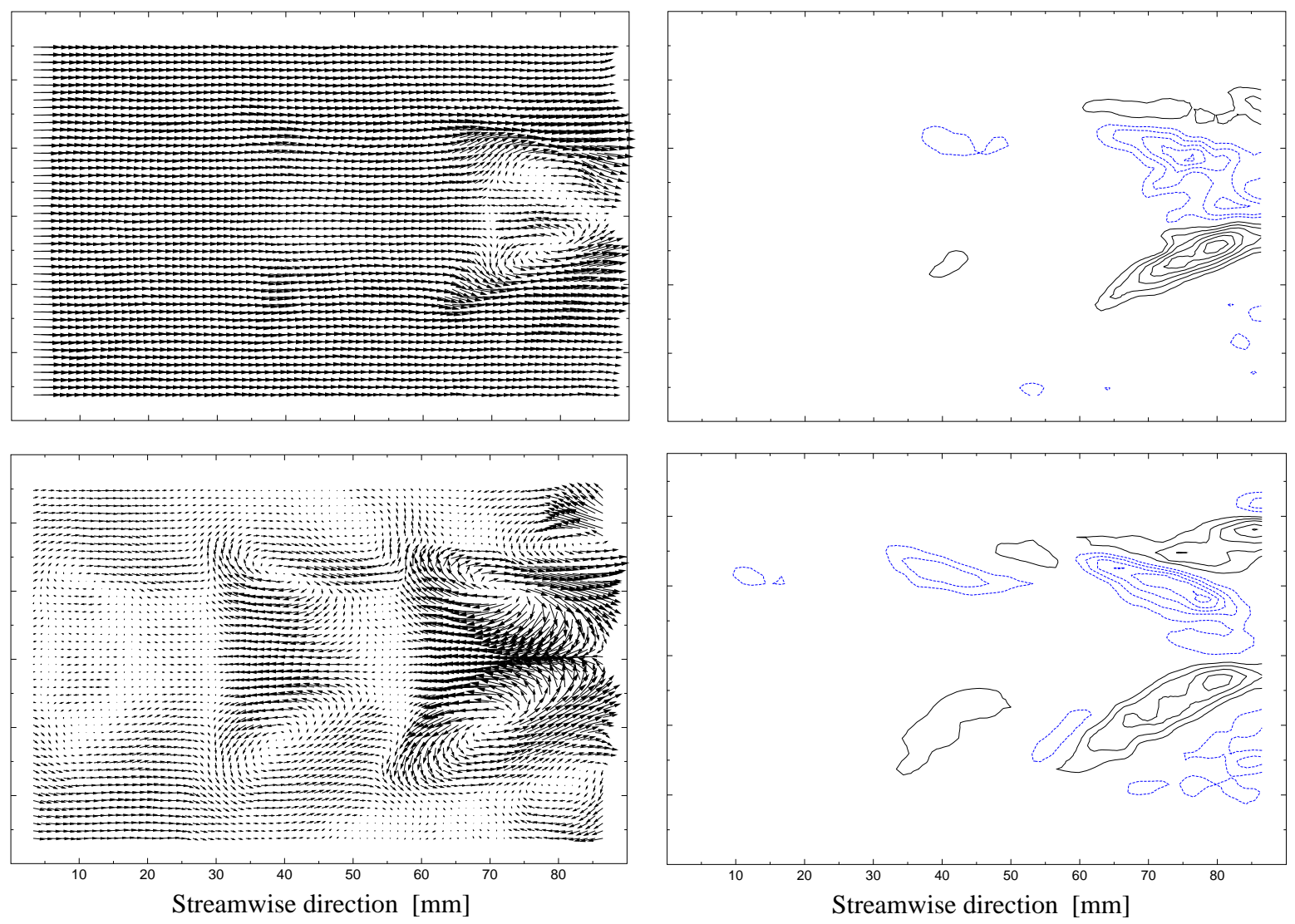

FIGURE 4.15: Instantaneous velocity vector field (left) and vorticity (right) with $\Lambda$-vortices recorded simultaneously in two planes parallel to the wall but at different heights (lower field closer to wall). The flow direction is from left to right.

an output frequency between $0.01 \mathrm{~Hz}$ and $1 \mathrm{MHz}$ was used. The width of each pulse is freely adjustable according to the specifications of the equipment used. The delay of all output trigger pulses with respect to the input trigger can be selected in the range from $50 \mathrm{~ns}$ up to $140 \mathrm{~s}$ with a resolution of $50 \mathrm{~ns}$ (jitter $12.5 \mathrm{~ns}$ ).

The mean displacement of corresponding particle-images was 8 pixel for $\Delta t=80 \mu \mathrm{s}$ and the height of the correlation coefficient was 0.6 for $32 \times 32$ pixel $^{2}$ interrogation windows. The evaluation technique of the stereoscopic images is based on the second order warping to ensure that the interrogation spots from each of a pair of stereoscopic images correspond to the same region of flow and the magnification is constant for all image positions [108]. For the evaluation of the data the FFT-based free shape cross-correlation was employed, which combines the advantages of the direct correlation (free-sized and free-shaped windows, high accuracy) and the simple FFT-based correlation (high speed evaluation), see section 2.4 for details. For sub-pixel accuracy the two dimensional Gaussian fit using the Levenberg-Marquardt method has been applied to find the position of the correlation peak and the Fisher transformation to determine the weight for each value, see section 2.4.2 for details. Figure 4.15 shows the in-plane velocity (left) and out-of-plane vorticity distribution (right) for two distances from the wall measured simultaneously. The flow direction is from left to right and a constant convection velocity of $5.75 \mathrm{~m} / \mathrm{s}$ has been subtracted from both vector fields in order to show the distribution of the velocity structures more clearly. The observation area started $265 \mathrm{~mm}$ 

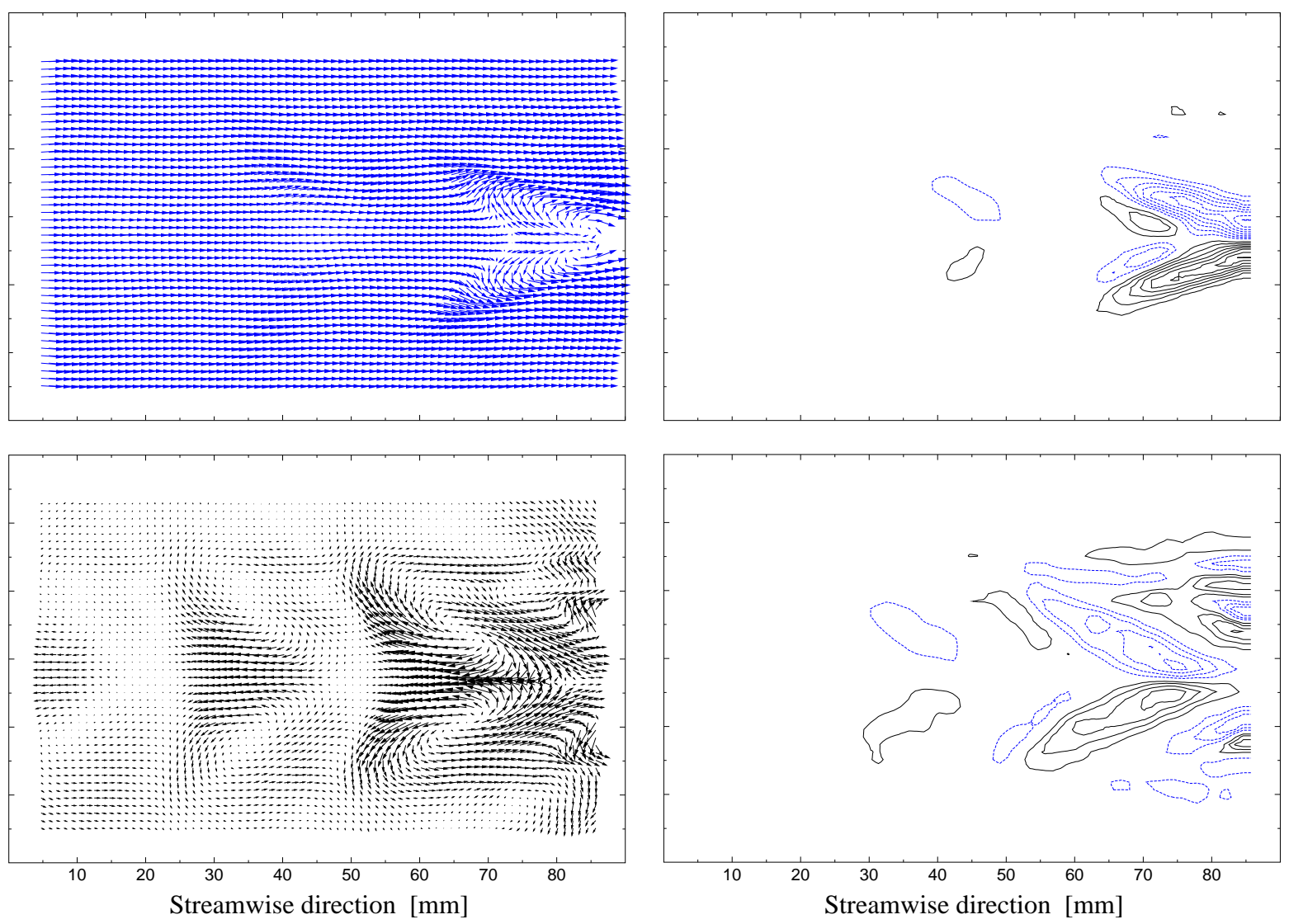

FIGURE 4.16: Instantaneous velocity (left) and vorticity (right) field with $\Lambda$-vortices recorded in two planes parallel to the wall but at different heights (lower field closer to wall) and separated in time.

behind the span-wise slot and the distance between the orthogonally polarised light-sheets is $0.5 \mathrm{~mm}$. The measurement position of the lower picture was at a height of $0.8 \mathrm{~mm}$ from the wall (the velocity fluctuations reach their maximum at this position) and the upper field was recorded $1.3 \mathrm{~mm}$ above the wall. This investigation is well suited to determine experimentally the orientation of the counter rotating vortex pair axis and its variation within the lifetime of the structure and the intensity of the acoustic excitation. As similar structures plays a dominant role in turbulent boundary layers according to chapter 1, the results are also instructive from this point of view, see [44] for example. Figure 4.16 shows velocity and vorticity fields in the same representation as figure 4.15 but the lower field was recorded $0.0013 \mathrm{~s}$ earlier and the intensity of the acoustic excitation was slightly increased.

This experiment demonstrates clearly the reliability and robustness of the measurement technique and numerical procedures that were developed to overcome the basic problems of existing PIV methods for the investigation of turbulent flows. In the following chapters the main turbulent boundary layer results measured with this technique will be outlined in detail. 


\section{Investigation of the xy-plane}

\subsection{The statistical description of turbulence}

The most striking characteristics of all turbulent flows is the complex spatio-temporal variation of the fluid mechanical variables. Even under stationary boundary conditions they fluctuate without any apparent regularity with different amplitude, wavelength and orientation [76]. From the present point of view the details of turbulent flows seem to be neither predictable nor reproducible because the finest details of the flow depend very much on the initial and boundary conditions, which are never known exactly, and in addition they are strongly affected by instabilities due to small disturbances. However, as the finest details of the turbulent motion are only of academic interest, because no serious practical application is conceivable where all details of the flow are required, the majority of information being present in a turbulent flow field can be reduced to a treatable form by applying the formalism of statistical mechanics [23, $64,65]$. The strength of this approach is well established by the kinetic theory of gases, where the effect of the molecular motion on the macroscopic fluid can be described by the kinematic viscosity $\nu$, provided the intermolecular distance $l_{\mathrm{m}}$, given by the mean free path length of the molecular gas, is much smaller than the Kolmogorov micro-scales $l_{\mathrm{k}}$ which represent the wavelength of the smallest macroscopic eddies, whose size depends only on the total energy $\epsilon$ and the viscosity.

$$
l_{\mathrm{m}} \ll l_{\mathrm{k}}=\left(\frac{\nu^{3}}{\epsilon}\right)^{1 / 4}
$$

In this approximation, the macroscopic fluid motion, represented by a fluid element, is considered as a large scale perturbation of the molecular chaos which becomes visible when the molecular motion is approximated by a continuous velocity field. In principle, this can be done by averaging the velocities of all molecules in the neighbourhood of a particular position. The basic feature of the statistical approach to turbulence is the transition from the consideration of a single turbulent flow to the consideration of the statistical ensemble of all similar flows. Each flow supplies a unique realization of the random variables (velocity $\underline{U}$, pressure $p$, density $\rho$ and temperature $T$ ) and each particular result represents one of $N$ possible results which might have occurred. For the characterisation of the physical phenomenon, the statistical theory supplies basically four quantities, namely the mean value, the variance, the probability density function and the auto-correlation function of the random variable. The mean value, defined in equation (5.2), can be seen as a static measure of the intensity of the signal and the variance, defined in equation (5.3), possesses information concerning the dynamics of the random signal under investigation. The probability density function, defined in equation (5.4), furnishes information about the properties of the data in the amplitude domain and the auto-correlation, 
defined in equation (5.5), yields comparable information in the frequency domain.

$$
\begin{aligned}
\langle U(\underline{r}, t)\rangle & =\frac{1}{N} \sum_{n=1}^{N} U_{n}(\underline{r}, t) \\
\left\langle u^{2}\right\rangle & =\frac{1}{N} \sum_{n=1}^{N} u_{n}^{2}(\underline{r}, t)=\frac{1}{N} \sum_{n=1}^{N}\left[U_{n}(\underline{r}, t)-\langle U(\underline{r}, t)\rangle\right]^{2} \\
\operatorname{PDF}(u) & =\lim _{\Delta u \rightarrow 0} \frac{P\left\{u<u_{n}(\underline{r}, t)<u+\Delta u\right\}}{\Delta u} \\
R_{u u}(\underline{r}, \Delta \underline{r}, t, \Delta t) & =\sum_{n=1}^{N} u_{n}(\underline{r}, t) u_{n}(\underline{r}+\Delta \underline{r}, t+\Delta t)
\end{aligned}
$$

When the relation between two random variables is considered, two additional functions become important namely the joint probability density function, defined in equation (5.6), and the normalised cross-correlation function, see equation (5.7). The joint probability density function describes the probability that two random variables will assume values within a certain range simultaneously and the cross-correlation function describes the dependence of one variable of a random process to the other process. This is important for the detection and recovery of deterministic data, which might be embedded in a random background, or for the determination of time delays or spatial shifts between two correlated random signals.

$$
\begin{gathered}
\operatorname{PDF}(u, v)=\lim _{\Delta u, \Delta v \rightarrow 0} \frac{P\left\{u<u_{n}<u+\Delta u \cap v<v_{n}<v+\Delta v\right\}}{\Delta u \Delta v} \\
R_{u v}(\underline{r}, \Delta \underline{r}, t, \Delta t)=\sum_{n=1}^{N} u_{n}(\underline{r}, t) v_{n}(\underline{r}+\Delta \underline{r}, t+\Delta t)
\end{gathered}
$$

The power of the statistical approach becomes evident when the basic conservation equations of mass, momentum and energy are reformulated by using the statistical functions. This approach, which allows the phenomena of turbulence to be viewed from different perspectives as only smoothly varying average quantities are considered, provides important relations like the well known law-of-the-wall the log-law or the five-third law, see [76, 89] for details. In case of a steady, two-dimensional flow along a flat plate for example, the propagation of the mean motion of an incompressible fluid of density $\rho$ and kinematic viscosity $\nu$ is described by the Reynolds equation in boundary layer approximation (5.8). It should be noted that in equation (5.8) the ensemble averaged values are replaced by the time averaged once for a better reading. This is always possible for stationary flows according to the ergodic theorem. $\bar{U}=U-u$ and $\bar{V}=V-v$ are the mean velocity components in stream-wise $(x)$ and wall-normal ( $y$ ) direction and $u$ and $v$ are the corresponding velocity fluctuations.

$$
\bar{U} \frac{\partial \bar{U}}{\partial x}+\bar{V} \frac{\partial \bar{U}}{\partial y}=U_{\infty} \frac{d U_{\infty}}{d x}+\frac{\partial}{\partial y}\left(\nu \frac{\partial \bar{U}}{\partial y}-\overline{u v}\right)-\frac{\partial}{\partial x}\left(\overline{u^{2}}-\overline{v^{2}}\right)
$$

This equation implies that for boundary layer flows only three components of the general Reynolds stress tensor $-\rho \overline{u_{i} u_{j}}$ are important for the turbulent mixing, namely $\rho \overline{u^{2}}, \rho \overline{v^{2}}$ and $-\rho \overline{u v}$. For attached flows without separation the stream-wise gradients of the normal stresses $\rho \overline{u^{2}}$ and $\rho \overline{v^{2}}$ in the second order term can be neglected as well relative to the shearing stress component $-\rho \overline{u v}$ because of the strong variation of the fluid mechanical quantities in wallnormal direction [89]. For the analysis of the turbulent mixing in wall bounded flows it is useful to differentiate between the following quantities as will be seen in the later. 


$$
\begin{array}{llll}
u>0 & \text { and } & v>0 & \mathrm{Q} 1 \text { or Outward interaction } \\
u<0 & \text { and } & v>0 & \mathrm{Q} 2 \text { or Ejection } \\
u<0 & \text { and } & v<0 & \mathrm{Q} 3 \text { or Inward interaction } \\
u>0 & \text { and } & v<0 & \mathrm{Q} 4 \text { or Sweep }
\end{array}
$$

The complexity of equation (5.8) can be further reduced by assuming that the production of turbulence, due to the conversion of the kinetic energy of the mean motion into turbulent fluctuations, is in equilibrium with the dissipation due to the molecular stresses:

$$
0=\frac{\partial}{\partial y}\left(\mu \frac{\partial \bar{U}}{\partial y}-\rho \overline{u v}\right) \quad \text { or } \quad \nu \frac{\partial \bar{U}}{\partial y}-\overline{u v}=\frac{\tau_{\mathrm{w}}}{\rho}
$$

As the turbulent shearing stress component $-\rho \overline{u v}$ becomes zero at the wall the following expression

$$
\lim _{y \rightarrow 0} \mu \frac{\partial \bar{U}}{\partial y}=\tau_{\mathrm{w}}
$$

can be used to determine the integration constant $\tau_{\mathrm{w}}$. With the friction velocity defined by $u_{\tau}=\sqrt{\tau_{\mathrm{w}} / \rho}$ and the assumption that $-\rho \overline{u v}=0$ holds, the following relation can be deduced from equation (5.13)

$$
\frac{\bar{U}}{u_{\tau}}=\frac{y u_{\tau}}{\nu}
$$

In the literature this so called law-of-the-wall is usually written in non dimensional wall-units $\bar{U}^{+}$and $y^{+}$defined by

$$
\bar{U}^{+}=\frac{\bar{U}}{u_{\tau}} \quad \text { and } \quad y^{+}=\frac{y u_{\tau}}{\nu}
$$

It is clear that the law-of-the-wall is only approximately valid in the vicinity of the wall $\left(y^{+}<\right.$ $5)$ where $-\rho \overline{u v}$ is small relative to the molecular stresses. This flow domain is usually called viscous sub-layer of the boundary layer [90]. Another important relation can be deduced from equation (5.13) by assuming that the molecular stresses are small relative to the turbulent ones. In this case $u_{\tau}^{2} \approx-\overline{u v}$ holds and the velocity profile becomes independent of the Reynolds number provided the velocities and spatial dimensions are normalised with $u_{\tau}$ and $\nu / u_{\tau}$. This is clear from the analysis of the dimensions because $d \bar{U} / d y$ can only depend on $u_{\tau}$ and $y$. Thus it follows from $d \bar{U} / d y \sim u_{\tau} / y$ with the von Kármán constant $\kappa$

$$
\bar{U}^{+}=\frac{1}{\kappa} \ln y^{+}+c
$$

This so called log-law is valid for $y^{+}>30$ until the effect of intermittency becomes important $(y / \delta>0.2)$. The region between $y^{+} \approx 5$ and $y^{+} \approx 30$ on the other hand, where the molecular and turbulent stresses are of equal order, is called buffer-layer.

For a deeper understanding of the turbulent motion two more equations need to be considered. The conversion of the kinetic energy of the mean motion given by equation (5.15) and 
(5.17), into turbulent fluctuations is described by the following equation, which follows from equation (5.8) after the multiplication with $\bar{U}$,

$$
\begin{aligned}
& \underbrace{\frac{1}{2}\left[\bar{U} \frac{\partial \bar{U}^{2}}{\partial x}+\bar{V} \frac{\partial \bar{U}^{2}}{\partial y}-U_{\infty} \frac{d U_{\infty}^{2}}{d x}\right]}_{\text {loss of kinetic energy of the mean motion }}-\underbrace{\left(\overline{u^{2}}-\overline{v^{2}}\right) \frac{\partial \bar{U}}{\partial x}-\overline{u v} \frac{\partial \bar{U}}{\partial y}}_{\text {production of turbulence }}+ \\
& +\underbrace{\frac{\partial}{\partial x}\left[\bar{U}\left(\overline{u^{2}}-\overline{v^{2}}\right)\right]+\frac{\partial}{\partial y}(\bar{U} \overline{u v})-\frac{\nu}{2} \frac{\partial^{2} \bar{U}^{2}}{\partial y^{2}}}_{\text {transfer of kinetic energy }}+\underbrace{\nu\left(\frac{\partial \bar{U}}{\partial y}\right)^{2}}_{\text {direct dissipation }}=0
\end{aligned}
$$

and the generation, transport and decay of $\overline{u v}$ can be derived from the Navier-Stokes equation when the equation for the $u$ component is multiplied by $v$ and added to the equation for the $v$ component multiplied by $u$ before averaging.

$$
\begin{aligned}
& \underbrace{\bar{U} \frac{\partial \overline{u v}}{\partial x}+\bar{V} \frac{\partial \overline{u v}}{\partial y}}_{\text {convection }}=\underbrace{\frac{1}{\rho p} \overline{p\left(\frac{\partial u}{\partial y}+\frac{\partial v}{\partial x}\right)}}_{\text {redistribution }}-\underbrace{\overline{v^{2}} \frac{\partial \bar{U}}{\partial y}}_{\text {production }} \\
& -\underbrace{\frac{\partial}{\partial y}\left(\overline{u v^{2}}+\overline{u p} / \rho\right)}_{\text {diffusion }}+\underbrace{\nu\left(\overline{v \nabla^{2} u}+\overline{u \nabla^{2} v}\right)}_{\text {dissipation }}
\end{aligned}
$$

Unfortunately, the remaining mathematical difficulty, associated with the statistical approach, is that the dynamical equations are under-determined due to the non-linearity in the momentum equations. In case of equation (5.8), for example, a relation between the mean motion $\bar{U}$ and all dominant components of the general Reynolds-stress tensor $\overline{u_{i} u_{j}}$ have to be assumed before a numerical integration can be performed. From the present point of view it seems unlikely that this so called closure problem can be solved from first principles, because most of the developed mathematical approximation methods fail for two reasons. Firstly, turbulent shear flows appear as a non-homogeneous, non-isotropic, multi-scale problem without any real scaling similarity ranking from the largest to the smallest scales. Secondly, turbulent flows are generally global and not local, which means that the local properties are not governed by the mean motion. For these reasons, it is evident that the functional dependence and statistical structure of $\overline{u_{i} u_{j}}$ must be determined experimentally. This will be the prime issue in the following.

\subsection{Experimental set-up}

The principle set-up of the double stereo-scopic PIV system utilised for the investigation in the wind-tunnel displayed on page 8 , is shown in figure 5.1. Because of the size of the large-scale structures in stream-wise direction and the relatively small velocity variations which must be properly resolved, the system is installed side by side. The actual observation angles $\alpha$ and distances $d_{o}$ along with the exact coordinates of each camera sensor, relative to the centre of the corresponding observation area, are displayed in table 5.2. For the registration and storage of the particle images four Peltier cooled high resolution PCO cameras with 1280 

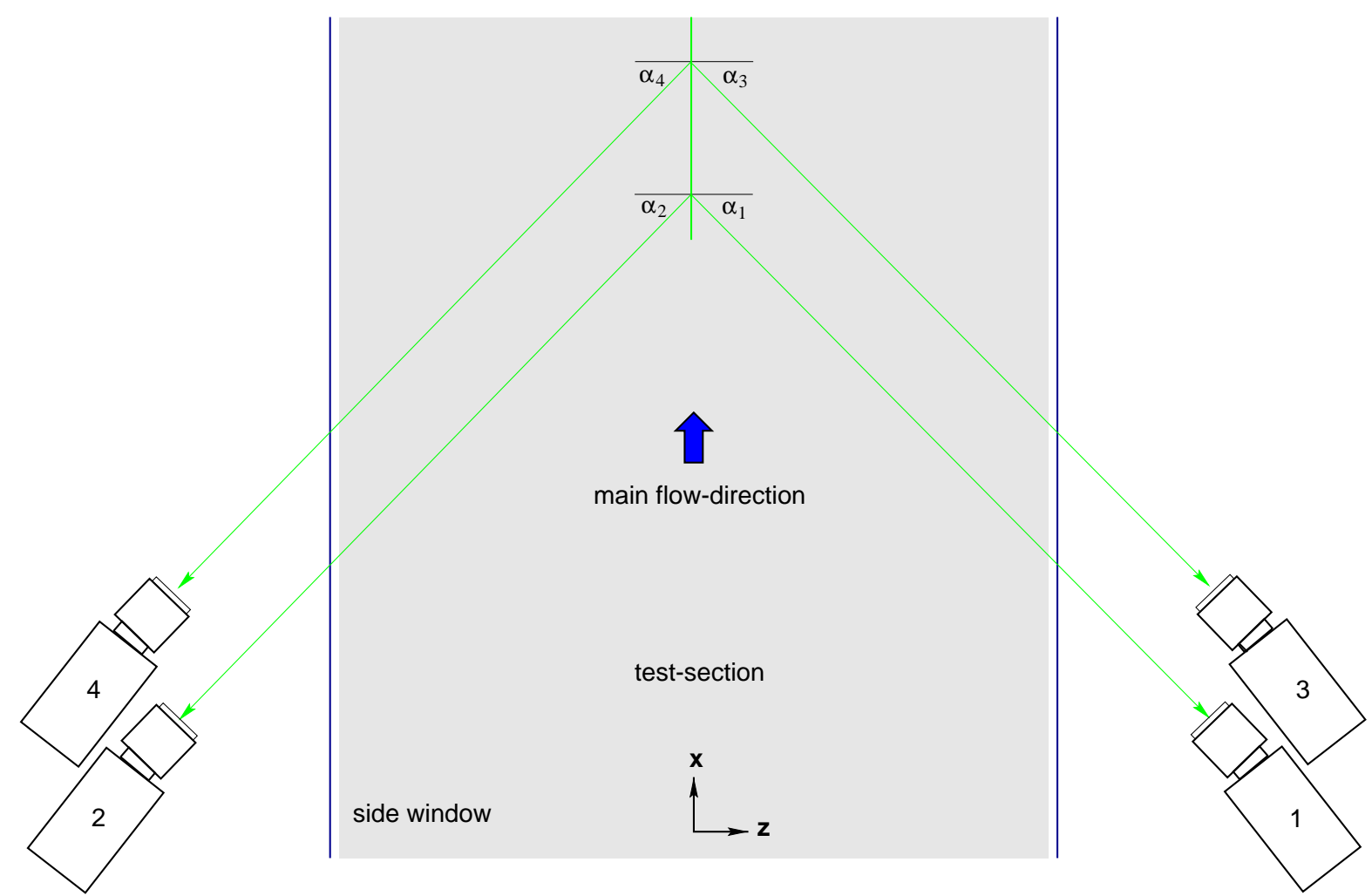

FIGURE 5.1: Schematic set-up of the double stereoscopic recording system installed $18 \mathrm{~m}$ behind the leading edge of the flat plate for the investigation in the stream-wise wall-normal plane (not in scale). Four digital cameras (1-4), mounted on Scheimpflug-adapters, observe the $2 \mu m$ tracer particles which are illuminated from below the test section $\left(90^{\circ}\right.$ scattering angle) by means of four synchronised lasers which deliver two intense green light pulses with $\lambda=532 \mathrm{~nm}$.

by 1024 pixel resolution and 12 Bit dynamic range have been employed, each connected with a Scheimpflug-adapter in order to adjust precisely the angle between the image-, objectand main-plane of the lens according to section 3.1.2. The imaging of the field of view was performed by means of $180 \mathrm{~mm}$ Carl Zeiss lenses with an aperture of 8 in order to avoid depth of focus effects and optical aberrations introduced by the $10 \mathrm{~mm}$ glass-walls of the windtunnel, see section 4.7. This allows to benefit from the high performance of the correlation algorithms, according to section 2.4, as the particle images appear as bright circles, 2-3 pixel in

\begin{tabular}{ccccc}
\hline camera & $x[\mathrm{~mm}]$ & $z[\mathrm{~mm}]$ & $d_{o}[\mathrm{~mm}]$ & $\alpha[\mathrm{deg}]$ \\
\hline 1 & -1109 & 1459 & 1838 & 37.5 \\
2 & -1109 & -1478 & 1848 & 36.9 \\
3 & -1051 & 1462 & 1801 & 35.7 \\
4 & -1038 & -1473 & 1802 & 35.2 \\
\hline
\end{tabular}

TABLE 5.1: Position and observation distance of the four cameras with respect to the centre of each field of view (intersection point of optical axis) and observation angles. Distance between centre of adjacent observation areas: $85 \mathrm{~mm}$. Distance between camera one and three: $156 \mathrm{~mm}$. 
diameter, surrounded by a dark background. The loss of light associated with this setting was compensated by combining the output beams of four independent Nd:YAG laser cavities (BMI model 5013 DNS 10), each approximately $255 \mathrm{~mJ}$ output energy per pulse at $\lambda=532 \mathrm{~nm}$ (see figure 4.2 on page 52) and by triggering the system in such a way that always two differently polarised pulses leave the laser-heads simultaneously. It is clear that always a delay of the order of the pulse length must exist between the two pulses to avoid laser induced damage of the frequency doubler crystal due to the high peak intensity, which might occur when the two beams interfere. Usually, this is automatically realized because the time accuracy of the PIV triggering systems is not suited to superimpose exactly two $1.5 \mathrm{~m}$ long light pulses $(5 \mathrm{~ns}$ pulse duration assumed) travelling with speed of $3 \times 10^{8} \mathrm{~m} / \mathrm{s}$. The $0.6 \mathrm{~mm}$ light-sheet was formed by three appropriate cylindrical and spherical lenses mounted in an optical bench and directed into the test-section by using a properly coated mirror, see section 4.6. To ensure that the measurement plane is aligned with respect to the stream-wise wall-normal plane, the cylindrical lens was rotated until the light-sheet reflection at the ceiling was parallel with a mark indicating the centreline of the wind-tunnel and the mirror was tilted in such a way that the weak light-sheet reflections, coming from the ceiling- and floor-window of the test-section, coincide on the mirror with the light-sheet coming from the optical system.

The tracer particles generated for this experiment were delivered from a smoke generator which produces high concentrations of monodisperse poly-ethylene-glycol particles with a mean diameter of $2 \mu \mathrm{m}$ according to figure 2.4 on page 18 or [47]. To obtain ideal conditions for accurate PIV measurements, the closed circuit wind-tunnel was completely seeded and continuously operated until no seeding inhomogeneities could be observed at all. During this procedure all diffuse wall reflections, caused by the interaction of the laser-light with tracer particles sticked on the wall, were removed with appropriate liquids and pressurised air while the wind-tunnel was running at a moderate speed. The operation of the wind-tunnel is essential, and should never be stopped before the experiment is finished in order to avoid any settling of the tracer particles which might deteriorate the performance of the measurements. As these particles follow the flow precisely, no settling of the particles or other diffuse reflections could be observed on the acquired data while the experiment was running. Only a tiny bright line of the wall reflection ( 3 pixel wide) was visible due to the glass / air interface. After the experiment, this line was detected by using correlation algorithms in order to determine the position of the wall with sub-pixel accuracy.

For the evaluation of the stereo-scopic images the second order warping technique, outlined in section 3.2, was applied along with the calibration validation procedure to ensure that the interrogation spots from each of a pair of stereo-scopic images correspond exactly to the same region of the flow, see section 3.3. The interrogation of the data was performed with the FFT-based free shape cross-correlation and for the peak detection with sub-pixel accuracy the two-dimensional Gaussian fit was applied. This peak finding method is less sensitive to sub-pixel displacements compared with the three point Gaussian peak fit, see figure 2.13 and 2.14. For the calculation of the velocity vectors $32 \times 32$ pixel and $64 \times 16$ pixel interrogation windows were selected. Because of the increased spatial resolution of the stretched windows in wall-normal direction spatial averaging effects could be easily estimated from the performed evaluation. It should be noted that an aspect ratio of 4 between the stream-wise and wall-normal dimensions of the measurement volume is relatively moderate regarding the typical values which are applied in numerical flow simulations. The evaluation of the data was performed in parallel on an eight processor high performance SGI computer, and a raid sys- 
tem was available for the storage of the data. The number of obviously incorrect vectors is on average less than $0.1 \%$ with the following conditions ( 0.1 pixel $<\Delta x<20$ pixel; -4 pixel $<$ $\Delta y<+4$ pixel; $\Delta x_{i}-\Delta x_{i+1}<5$ pixel), the average height of the correlation coefficient is above 0.8 and the bandwidth of particle images displacements varies between zero at the wall and 18 pixel on average for a light pulse delay of $\Delta \tau=300 \mu \mathrm{s}$ for the $3 \mathrm{~m} / \mathrm{s}$ investigation and $\Delta \tau=110 \mu \mathrm{s}$ for the $7 \mathrm{~m} / \mathrm{s}$ case. The following table summarises the basic information needed for the characterisation of the experiment.

\begin{tabular}{lrrl}
\hline$R e_{\theta}$ & 7800 & 15000 & {$[1]$} \\
$R e_{\delta}$ & $7.4 \times 10^{4}$ & $16 \times 10^{4}$ & {$[1]$} \\
$R e_{x}$ & $3.6 \times 10^{6}$ & $8.4 \times 10^{6}$ & {$[1]$} \\
\hline$U_{\infty}$ & 3 & 7 & {$[\mathrm{~m} / \mathrm{s}]$} \\
$u_{\tau}$ & 0.121 & 0.263 & {$[\mathrm{~m} / \mathrm{s}]$} \\
$\delta$ & 0.37 & 0.34 & {$[\mathrm{~m}]$} \\
$\delta^{+}$ & 3000 & 5980 & {$[1]$} \\
field of view & $160 \times 52$ & $160 \times 52$ & {$\left[\mathrm{~mm}^{2}\right]$} \\
field of view & $0.44 \times 0.14$ & $0.47 \times 0.15$ & {$\left[\delta^{2}\right]$} \\
field of view & $1298 \times 421$ & $2812 \times 914$ & {$\left[\Delta x^{+} \Delta y^{+}\right]$} \\
spatial resolution & $3.6 \times 0.9 \times 0.6$ & $1.8 \times 1.8 \times 0.6$ & {$\left[\mathrm{~mm}^{3}\right]$} \\
spatial resolution & $28 \times 7 \times 5$ & $31.8 \times 31.8 \times 10.5$ & {$\left[\Delta x^{+} \Delta y^{+} \Delta z^{+}\right]$} \\
pulse separation & 300 & 110 & {$[\mu \mathrm{s}]$} \\
dynamic range & $1.15<\Delta x<12.6$ & $0.1<\Delta x<18$ & {$[\mathrm{pixel}]$} \\
\hline vectors per sample & 4592 & 4644 & \\
number of samples & 2450 & 2450 & \\
\hline
\end{tabular}

TABLE 5.2: Relevant parameter for the characterisation of the experiment performed $18 \mathrm{~m}$ behind the leading edge of the flat plate in the $x y$-plane of the turbulent boundary layer flow.

\subsection{Statistical properties of the flow}

\subsubsection{Single point statistics}

To validate the flow characteristics and the performance and accuracy of the double stereoscopic PIV system, the main statistical properties of the turbulent boundary-layer flow, measured $18 \mathrm{~m}$ behind the leading edge of the flat plate in the flow facility displayed on page 8 , were calculated from the velocity data and compared with the theoretical results presented in section 5.1 and the hot-wire anemometry measurements in $[11,21]$. The hot-wire measurement was performed by Bruns, Dengel and Fernholz in 1992 in the large wind-tunnel at the Hermann Föttinger Institute (HFI) in Berlin at a similar Reynolds number but different $x$ and $U_{\infty}$. The measurement position was $6.7 \mathrm{~m}$ behind a $\mathrm{V}$-dymotape tripping device and the thickness of the boundary layer at the measurement location was $\delta \approx 0.174 \mathrm{~m}$ and $0.153 \mathrm{~m}$ at free stream velocities of $U_{\infty} \approx 11 \mathrm{~m} / \mathrm{s}$ and $30 \mathrm{~m} / \mathrm{s}$. The corresponding Reynolds number based on the momentum thickness $\theta$ was comparable with the PIV experiments listed in table 5.2, 


\section{Investigation of the $x y-p l a n e$}

namely $R e_{\theta} \approx 7140$ and 16080 . The friction velocity $u_{\tau}=U_{\infty} \sqrt{c_{\mathrm{f}} / 2}$ was determined from the parameter $\sqrt{c_{\mathrm{f}} / 2}$ in equation (5.20) to $0.42967 \mathrm{~m} / \mathrm{s}$ and $1.08187 \mathrm{~m} / \mathrm{s}$.

$$
\frac{\bar{U}}{U_{\infty}}=2.44 \sqrt{\frac{c_{\mathrm{f}}}{2}} \ln \left[\frac{U_{\infty} y}{\nu} \sqrt{\frac{c_{\mathrm{f}}}{2}}\right]+5.1 \sqrt{\frac{c_{\mathrm{f}}}{2}}
$$

Figure 5.2 reveals the mean velocity profile of the turbulent boundary layer flow in outer- and inner-law scaling. The agreement between the stereoscopic PIV results with the analytical law-of-the-wall and the log-law, derived on page 71, is excellent and also the hot-wire measurements match nicely. Only for $y^{+}>800$ a progressing departure of the HWA results from the present PIV investigation can be observed in the semilogarithmic representation. This effect is caused by the fact that the boundary layers thickness at HFI is only half of the thickness obtained at LML. However, this is not relevant for the present investigation, because here only the near-wall phenomena below $y / \delta<0.15$ will be considered and not the outer-flow effects caused by intermittency. Remarkable is the average dynamic which could be reached with the PIV-system and the spatial resolution in wall-normal direction. The location of the first measurement point, located at $y^{+} \approx 2.3$, is comparable with the nearest wall location
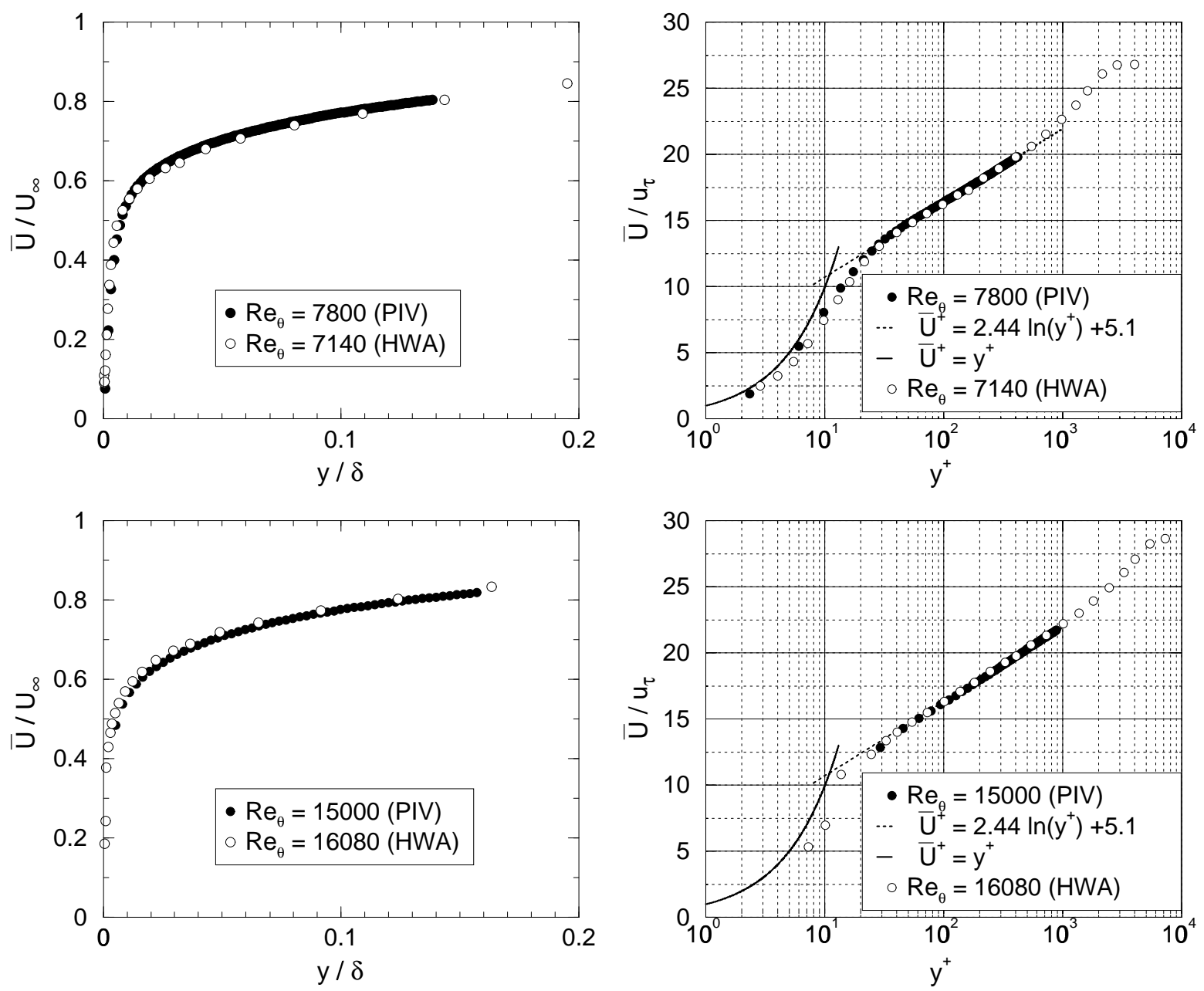

FIGURE 5.2: Comparison of the measured mean velocity profiles in outer- (left) and inner-law scaling (right) with the hot-wire results by Bruns et al $[11,21]$ for two Reynolds numbers. 
for the stream-wise velocity in [55] but here the velocity could be determined directly without a sophisticated calibration procedure as required in case of the hot-wire investigation to compensate the additional heat-loss induced by the solid wall. When the wall-normal velocity component is considered, the first measurement point obtained with the hot-wire technique is farther away from the wall by one order of magnitude, compared with the present PIV investigation, due to the orientation and size of the sensor applied in [55]. The domain covered by the PIV investigation is nearly constant when considered in outer variables $(0<y / \delta<0.15)$ because of the weak dependence of the boundary-layer thickness $\delta$ on the Reynolds number. However, when considered in inner variables on the other hand, it can be seen that the investigation at $R e_{\theta}=7800$ roughly covers the domain from $3<y^{+}<400$ and at $R e_{\theta}=15000$ the range $30<y^{+}<900$, because the log-law region increases in thickness with increasing Reynolds number when considered in wall-units. This allows to examine the near-wall structure at $R e_{\theta}=7800$, Reynolds number effects within the logarithmic region between $30<y^{+}<400$ and large-scale flow structures at $R e_{\theta}=15000$. The size of the region, where
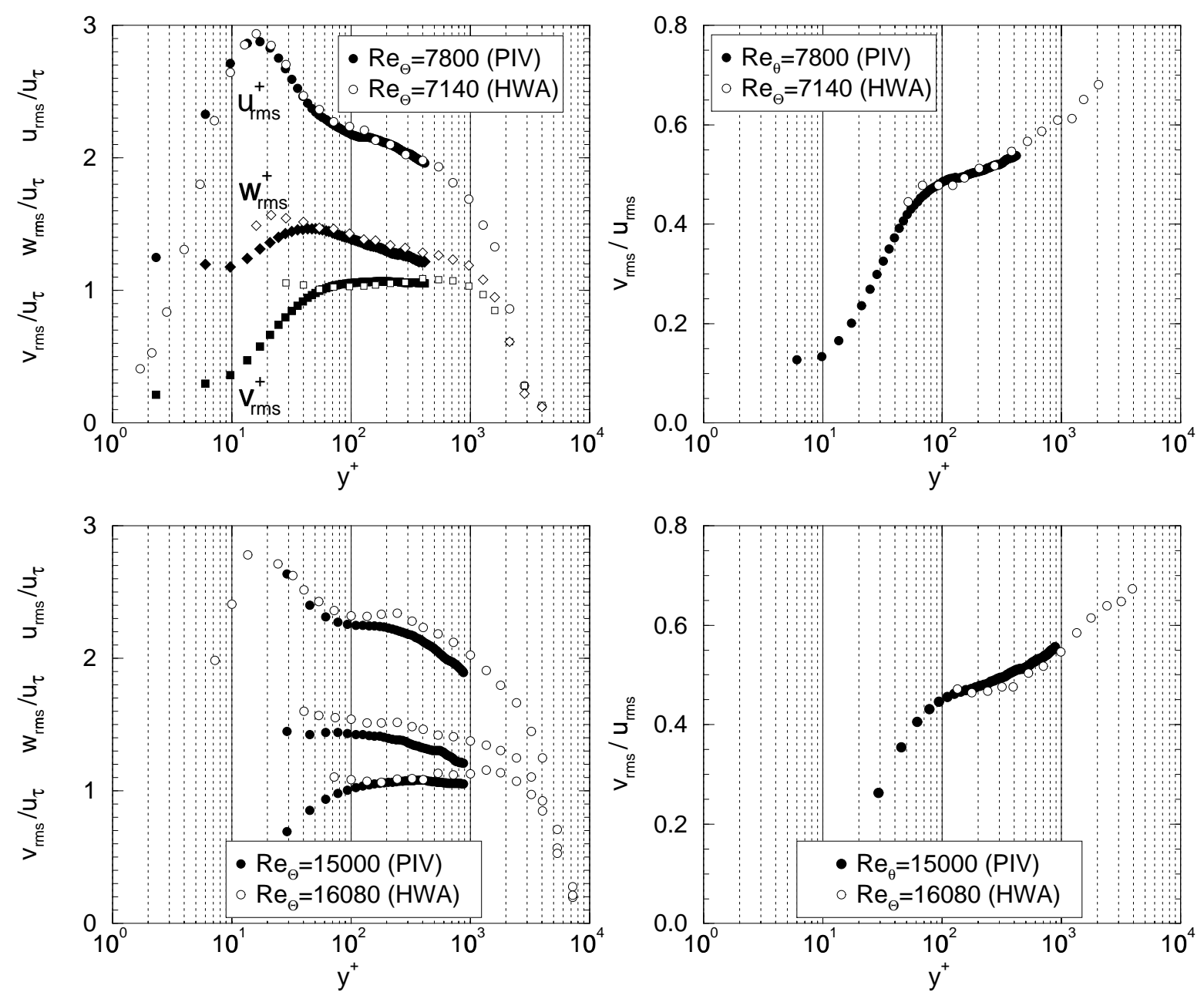

FIGURE 5.3: Left: Non-dimensional rms-profiles of the three velocity fluctuations for $\operatorname{Re}_{\theta} \approx 7800$ (top) and $\operatorname{Re}_{\theta} \approx 15000$ (bottom). Right: Dependence of the anisotropy parameter $\left(\overline{v^{2}} / \overline{u^{2}}\right)^{1 / 2}$ on the wall coordinate in inner-law scaling for both Reynolds numbers. 
the log-law holds, is quite large for both cases so that a direct interaction between the nearwall flow structure with the intermittent flow region can not take place. Thus the turbulent flow state can be considered as fully developed. Fully developed flows are characterised by a Kolmogorov cascade including an inertial range over at least one order of magnitude in the wavenumber space [78]. This condition implies unsteadiness, rotational, three-dimensionality, non-deterministic, diffusion and dissipation, e.g. attributes which are generally referred to characterise turbulent flows. The dynamic of the velocity signal as a function of the wall distance can be estimated from figure 5.3 which shows the normalised rms values of the three velocity fluctuations for both flow cases. It can be seen that the maximum can be properly resolved with state-of-the-art PIV systems, provided the magnification of the imaging system is well adjusted relative to the flow structures and the problems associated with the strong wall reflections are solved. The low Reynolds number results agree quite well with the HWA measurements kindly provided by Prof. Dr. Fernholz. Only the first two measurement points near the wall are overestimated. This is natural due to the finite size of the measurement volume. When the high Reynolds number results are considered the deviation increases but the increase of the second peak with increasing Reynolds number is clearly visible and in agreement with the literature [21]. To validate the degree of anisotropy between different velocity components in turbulent shear flows, the ratio between orthogonal velocity fluctuations is shown in the right graphs of the same figure. As the energy from the mean motion is first transferred into the stream-wise velocity fluctuation before the transfer into the $v$ and $w$ component takes place by means of pressure fluctuations, the value of the anisotropy parameter is usually around 0.6 for large wall distances. In addition, it can be seen that $\left(\overline{v^{2}} / \overline{u^{2}}\right)^{1 / 2}$ increases gradually with the wall distance. Figure 5.4 shows the turbulence-level defined as $\left(\overline{u^{2}}\right)^{1 / 2} / \bar{U}$ for both flow cases. The agreement with the hot-wire measurements performed at $R_{\theta}=7140$ and 16080 is excellent. Only the first PIV measurement point in the left graph is far away from the HWA result, which is around $T u=0.4$ for $y^{+}<5$. However, this is associated with the increasing measurement error when the displacement of the particle images becomes very small, see chapter 2 for details.
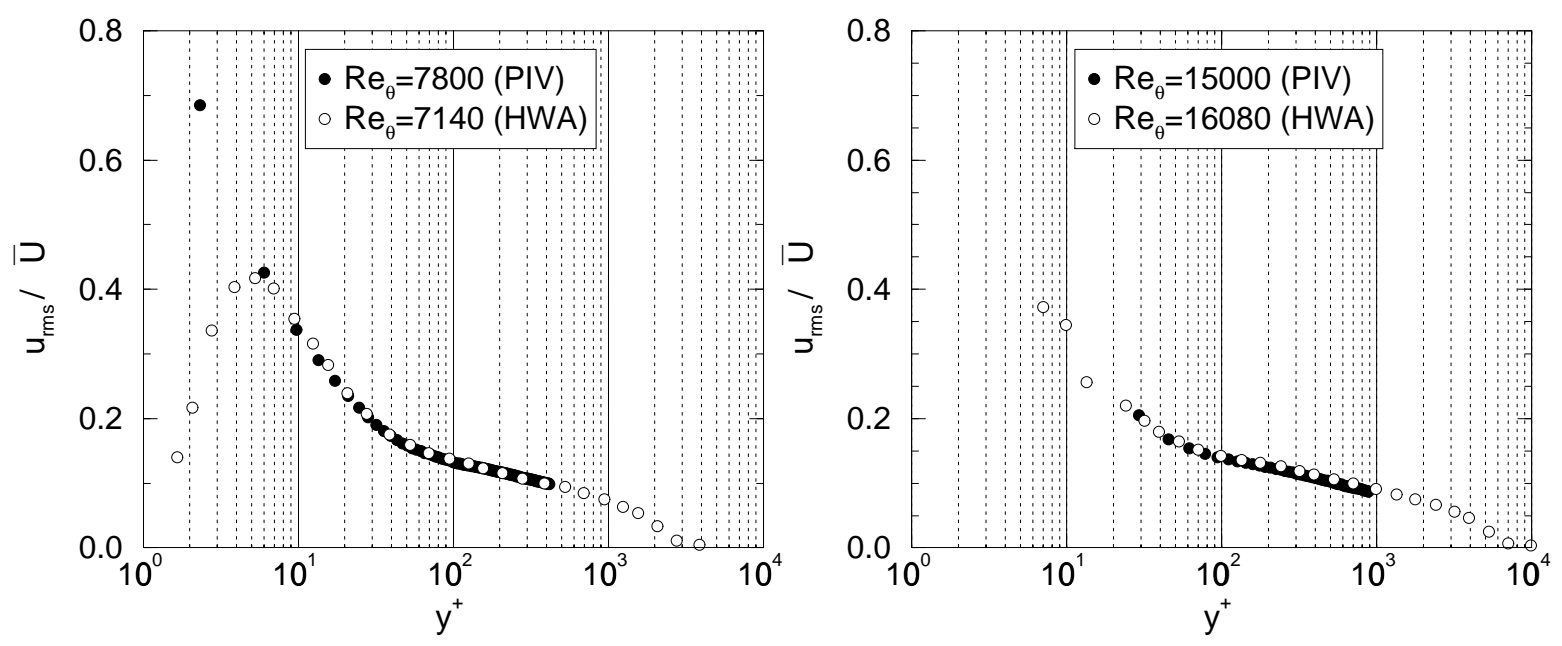

FIGURE 5.4: Turbulence-level $T u=\sqrt{\overline{u^{2}}} / \bar{U}$ measured at two Reynolds numbers. 


\subsubsection{Spatial auto- and cross-correlation functions}

It is obvious that the single point statistics, presented in the previous section, do not cover any aspects associated with the organised flow motion outlined in chapter 1. However, it is shown in section 5.1 that for the turbulent mixing in wall-bounded flows this coherent motion must be of primary importance. This can be deduced from the presence of the single point correlations terms $\overline{u^{2}}, \overline{v^{2}}$ and especially $-\overline{u v}$ in equation (5.8). For this reason the spatial correlations of the velocity fluctuations, introduced in section 5.1, will be investigated in this section. The investigation of the spatial correlations of the velocity fluctuations furnishes quantitative information about the average dimensions of the turbulent flow structures and allows to deduce several length and time scales with physical meaning such as the dissipation or the integral length scales, which play a dominant role in the transport equations derived in [90]. However, due to the anisotropic nature of wall-bounded flows according to figure 5.3, the length scales are not uniquely defined as in the case of isotropic turbulence. In addition, it should be kept in mind that the general investigation of the correlation function might be quite complex in shear-flows because this function depends on the flow variables considered, as well as their mutual location, separation and time delay. For simplicity, only the primary spatial correlations $R_{u u}, R_{v v}$ and $R_{w w}$ of the velocity fluctuation will be considered in the following, along with some double and triple correlations which are important for the turbulent mixing according to equation (5.20). The dependence of the primary correlations in a turbulent boundary layer along the three coordinates was extensively investigated by Grant in 1958 by using a pair of hot-wire probes $2 \mathrm{~m}$ behind a trip fence at $U_{\infty}=6.25 \mathrm{~m} / \mathrm{s}$ and $\delta=55 \mathrm{~mm}$. Although he could confirm the high degree of order present in the flow field by analysing the large-scale dependence of various correlations along the principle coordinates, he was forced to conclude that the various correlations are incompatible with the idea of energetic eddy structures convecting downstream. Since it is impossible to predict a unique set of vortical structures from the correlations, the motivation for this conclusion was based on the different size of the primary correlations in stream-wise direction and the small extend of $R_{v v}$ along the span-wise one. However, due to the small thickness $\delta$ of the boundary layer, it is not yet evident if this strong difference in the various correlations is a real shear-flow property or maybe caused by the entraining motions, which do not belong to the main turbulence structure. For this reason the present investigation focuses on the functional dependence of the spatial correlations in the near-wall region and their variation with the Reynolds number. Of particular interest is the correlation in the vicinity of the maximum, as this information is difficult to achieve by using intrusive measurement techniques because the signal from a single probe is affected by approaching the second probe and the values along the stream-wise axis is biased because the measurement values of the down-stream probe are affected by the wake of the upstream probe as pointed out in [24, 100].

In the following the size and shape of the primary correlation functions will be analysed because these values furnish the information about the average dimensions of the moving flow structures, their degree of organisation and their propagation direction relative to the mean motion. This becomes obvious when figure 5.5 and figure 5.6 are considered which reveal the primary correlation of the stream-wise velocity fluctuations for various wall distances of the fixed point (compare location of the maximum or the $y^{+}$value in the legend) at $R e_{\theta}=7800$ and 15000 . The contours of the following plots are spaced in intervals of 0.05 for the primary correlation (excluding 0.05 and 1) and the position and amplitude of the minimum and maximum are included for comparison. Continuous lines indicate positive correlation. Clearly 

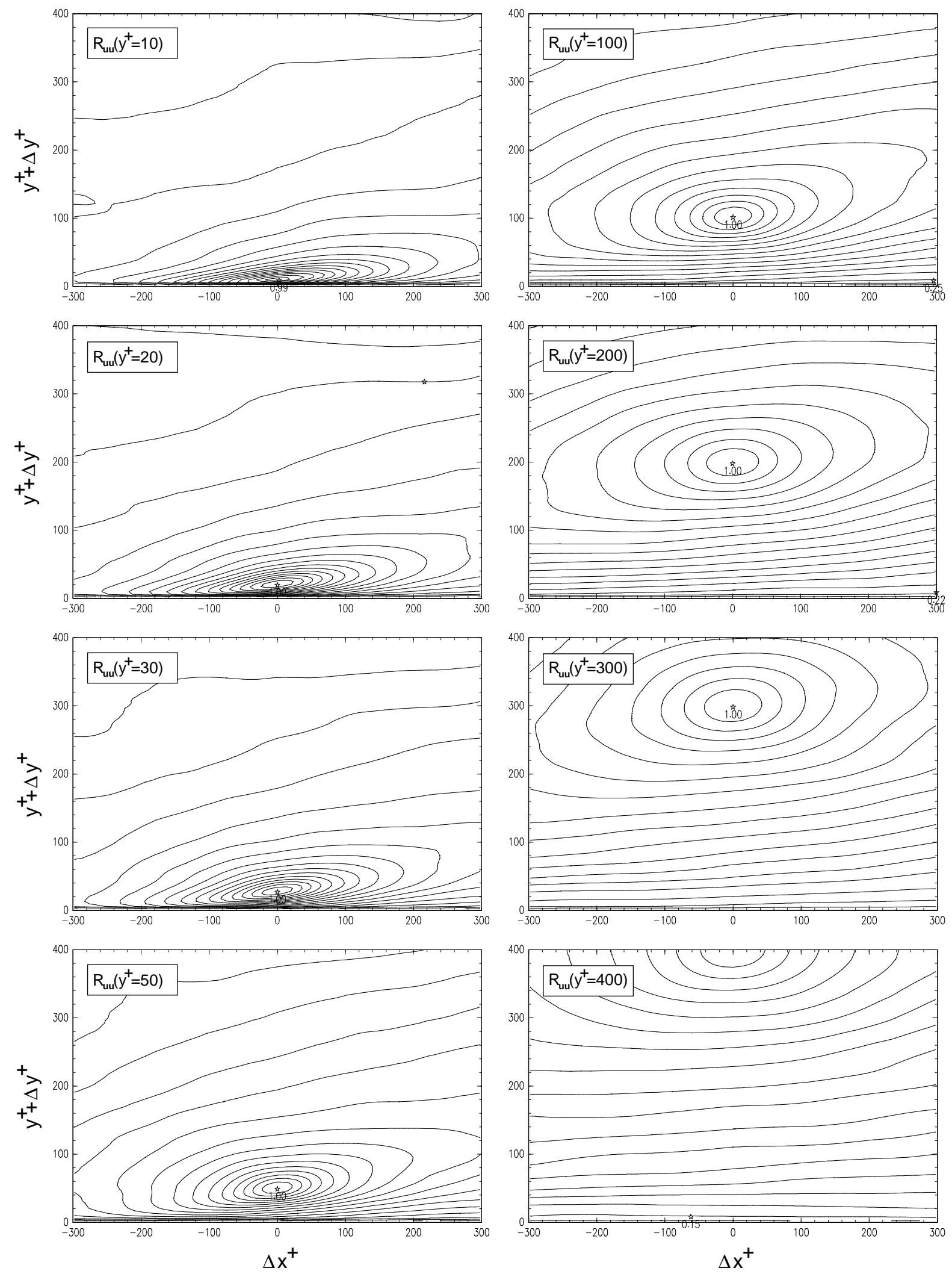

FIGURE 5.5: $R_{u u}\left(\Delta x^{+}, y^{+}, y^{+}+\Delta y^{+}\right)$for various $y^{+}$-locations of the fixed point (see legend) measured at $R e_{\theta}=7800$. 

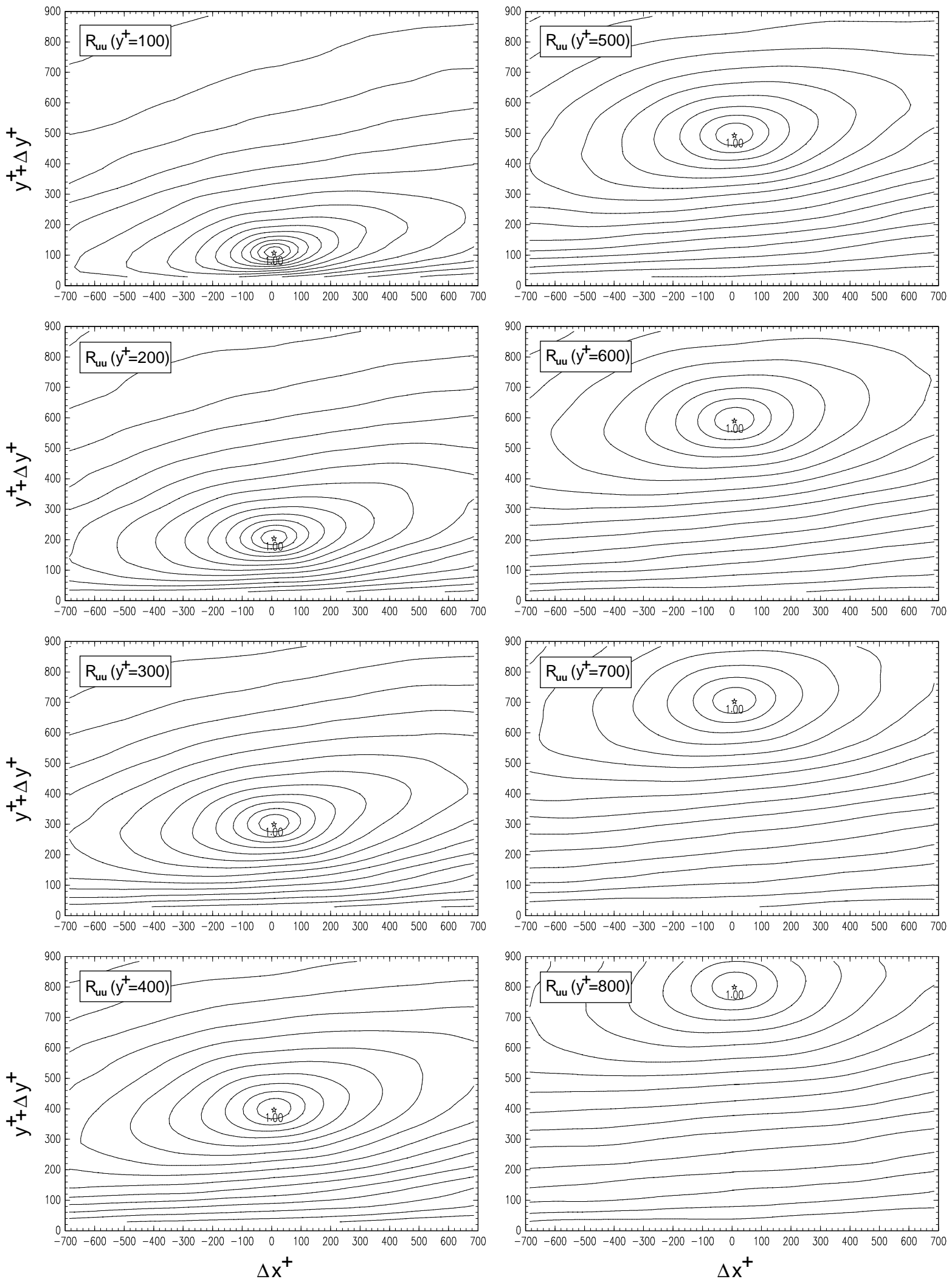

FIGURE 5.6: $R_{u u}\left(\Delta x^{+}, y^{+}, y^{+}+\Delta y^{+}\right)$for various $y^{+}$-locations of the fixed point (see legend) measured at $R e_{\theta}=15000$. 

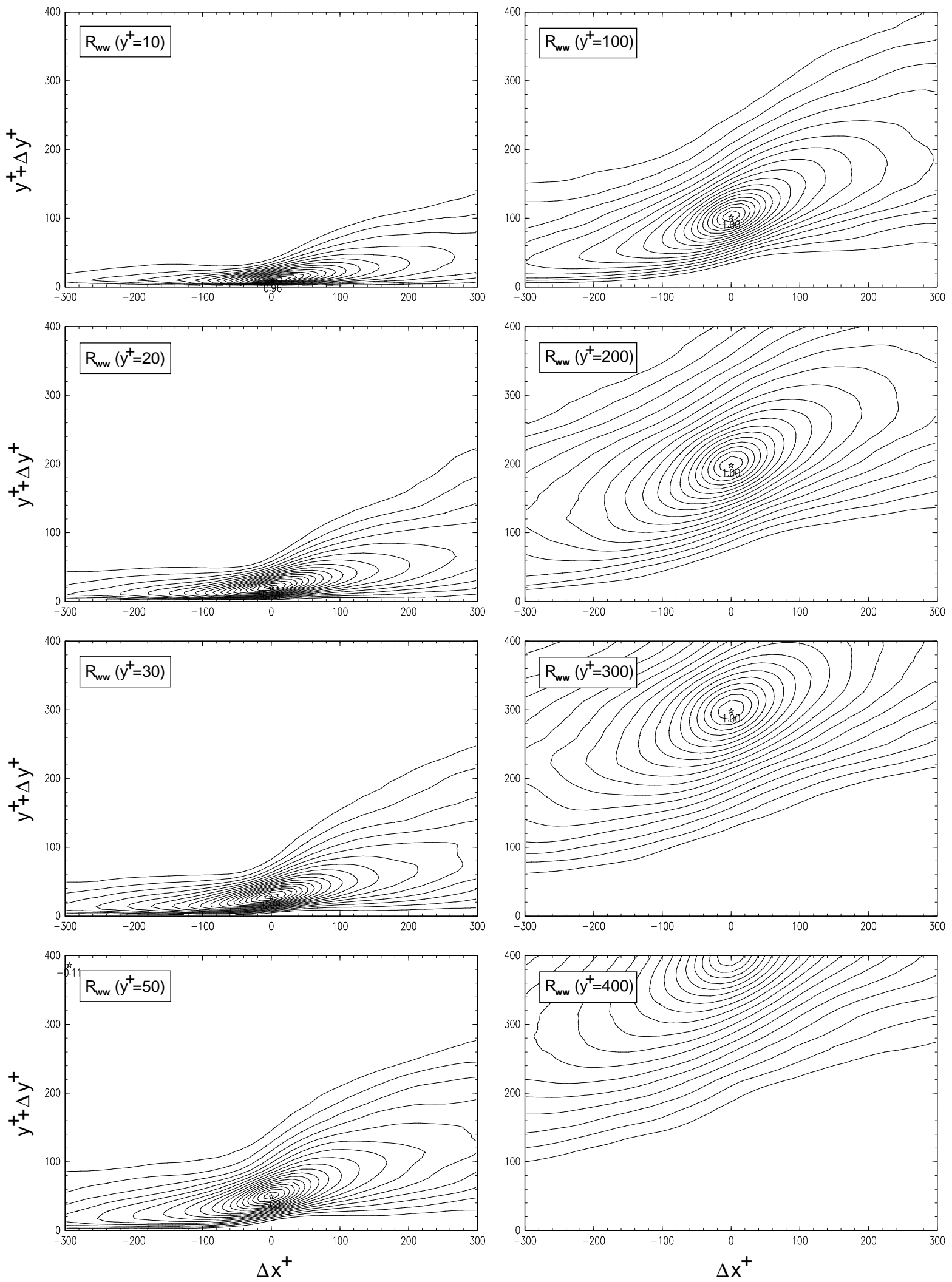

FIGURE 5.7: $R_{w w}\left(\Delta x^{+}, y^{+}, y^{+}+\Delta y^{+}\right)$for various $y^{+}$-locations of the fixed point (see legend) measured at $R e_{\theta}=7800$. 

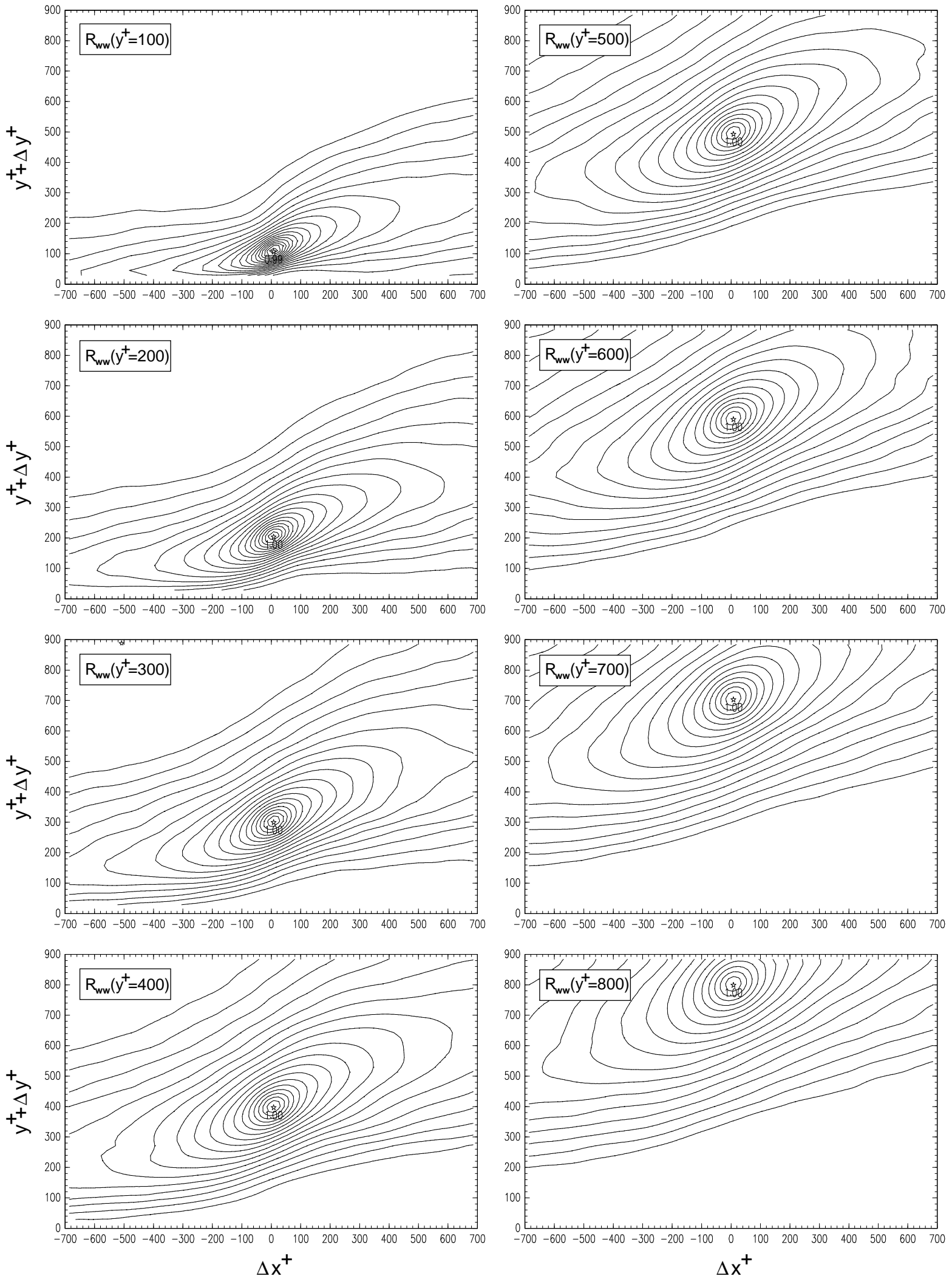

FIGURE 5.8: $R_{w w}\left(\Delta x^{+}, y^{+}, y^{+}+\Delta y^{+}\right)$for various $y^{+}$-locations of the fixed point (see legend) measured at $R e_{\theta}=15000$. 
visible is the elliptical shape of all correlated area, with the principal axis slightly inclined with respect to the $x$-axis, and the variation of the correlation size from the exact location of the fixed point. The elliptical shape of the structures and their inclination can be explained by the strong gradients in wall-normal direction, which tend to elongate the flow pattern (a circular cloud embedded in a shear flow for instance would appear elliptical and tilted after a short time). Reynolds number effects can be investigated by comparing the right column of figure 5.5 with the left column of figure 5.6 as the wall locations of the fixed point is identical in viscous units $\left(y^{+}=100,200,300,400\right)$. Qualitatively, the agreement is quite good when the scale in the legend is considered. Figure 5.7 and figure 5.8 show the functional dependence of the $R_{w w}$ correlation on the Reynolds number and on the location of the fixed point. The two-dimensional structure of this correlation function reveals surprising features which were not known previously. Especially the different dependence of the correlation in up-stream and down-stream direction on the fixed-point should be noted as well as the incli-
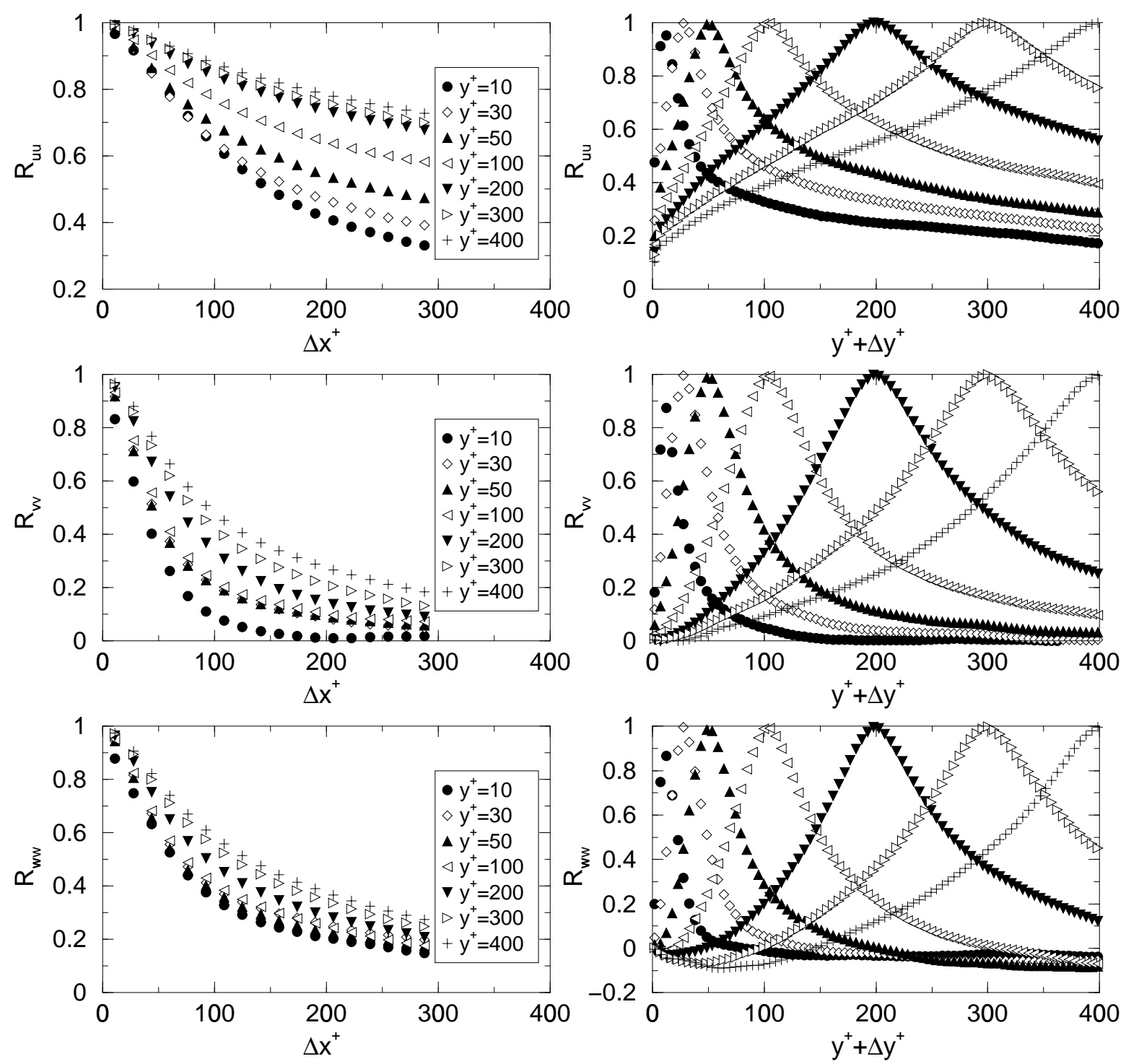

FIGURE 5.9: $R_{u u}\left(\Delta x^{+}, y^{+}, y^{+}+\Delta y^{+}\right), R_{v v}\left(\Delta x^{+}, y^{+}, y^{+}+\Delta y^{+}\right)$and $R_{w w}\left(\Delta x^{+}, y^{+}, y^{+}+\Delta y^{+}\right)$ measured at $R e_{\theta}=7800$. 
nation of the structure around the maximum of correlation and further away from this point. As this correlation appears crestfallen, two different angles of attack can be estimated when moving on the correlation ridge in up-stream and down-stream direction, and it can be seen that the latter one is larger with respect to the other one for all locations of the fixed point. The similarity between the size of $R_{u u}$ and $R_{w w}$ in stream-wise direction and the elliptical shape around the maximum, accompanied by the general variation of the angle with increasing wall distance of the fixed point, implies a relation between both fluctuations. This might be a result of a complex velocity structure (not necessarily a vortex structure) moving with the fluid, but the exact relation cannot be seriously deduced from the correlation functions. However, when the one-dimensional representations along the two coordinates through the maximum of correlation in figure 5.9 and figure 5.10 are compared, this similarity vanishes as the orientation of the principal axis of the ellipse around the span-wise coordinate differs
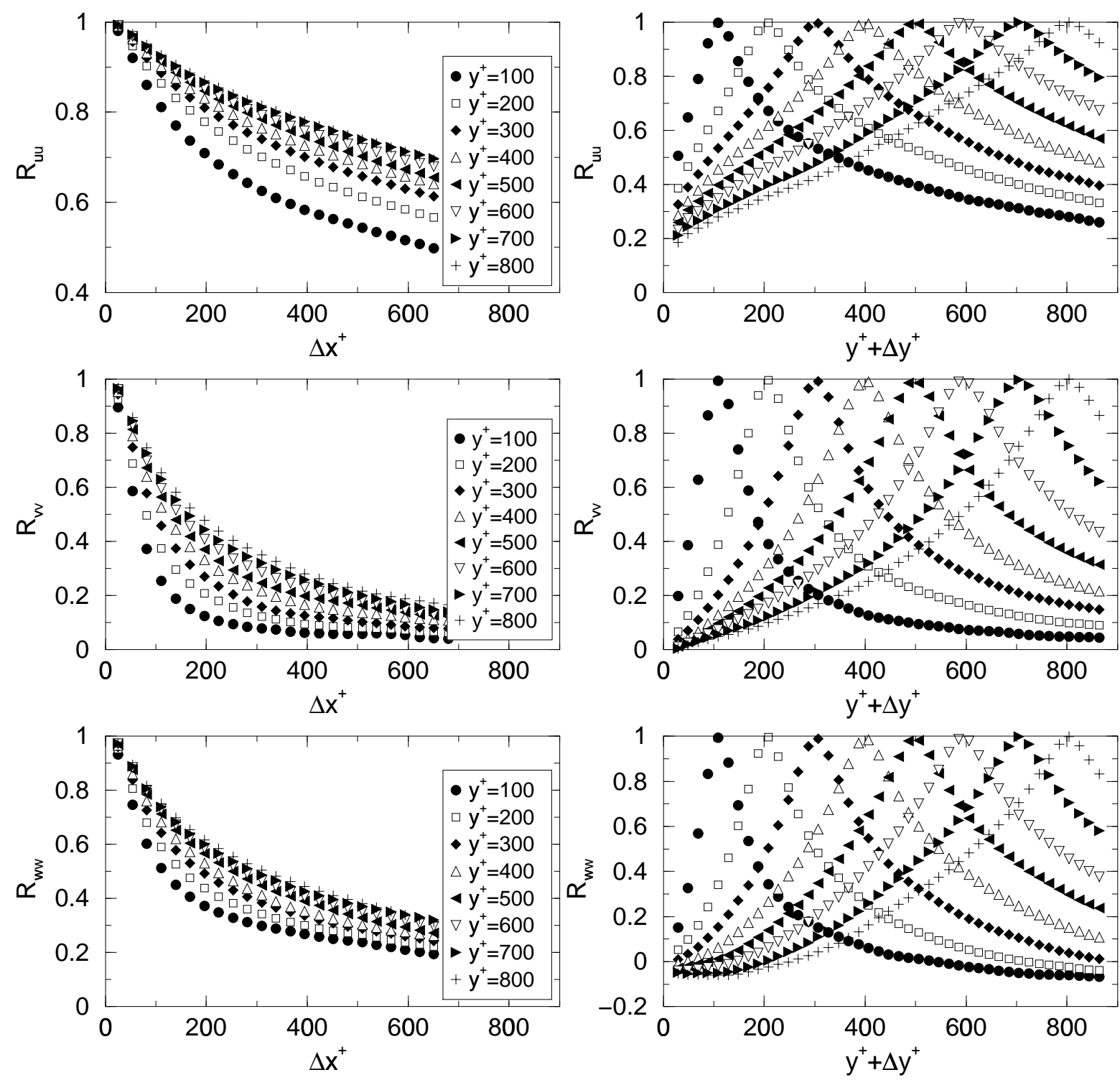

FigURE 5.10: $R_{u u}\left(\Delta x^{+}, y^{+}, y^{+}+\Delta y^{+}\right), R_{v v}\left(\Delta x^{+}, y^{+}, y^{+}+\Delta y^{+}\right)$and $R_{w w}\left(\Delta x^{+}, y^{+}, y^{+}+\Delta y^{+}\right)$ measured at $R e_{\theta}=15000$. 

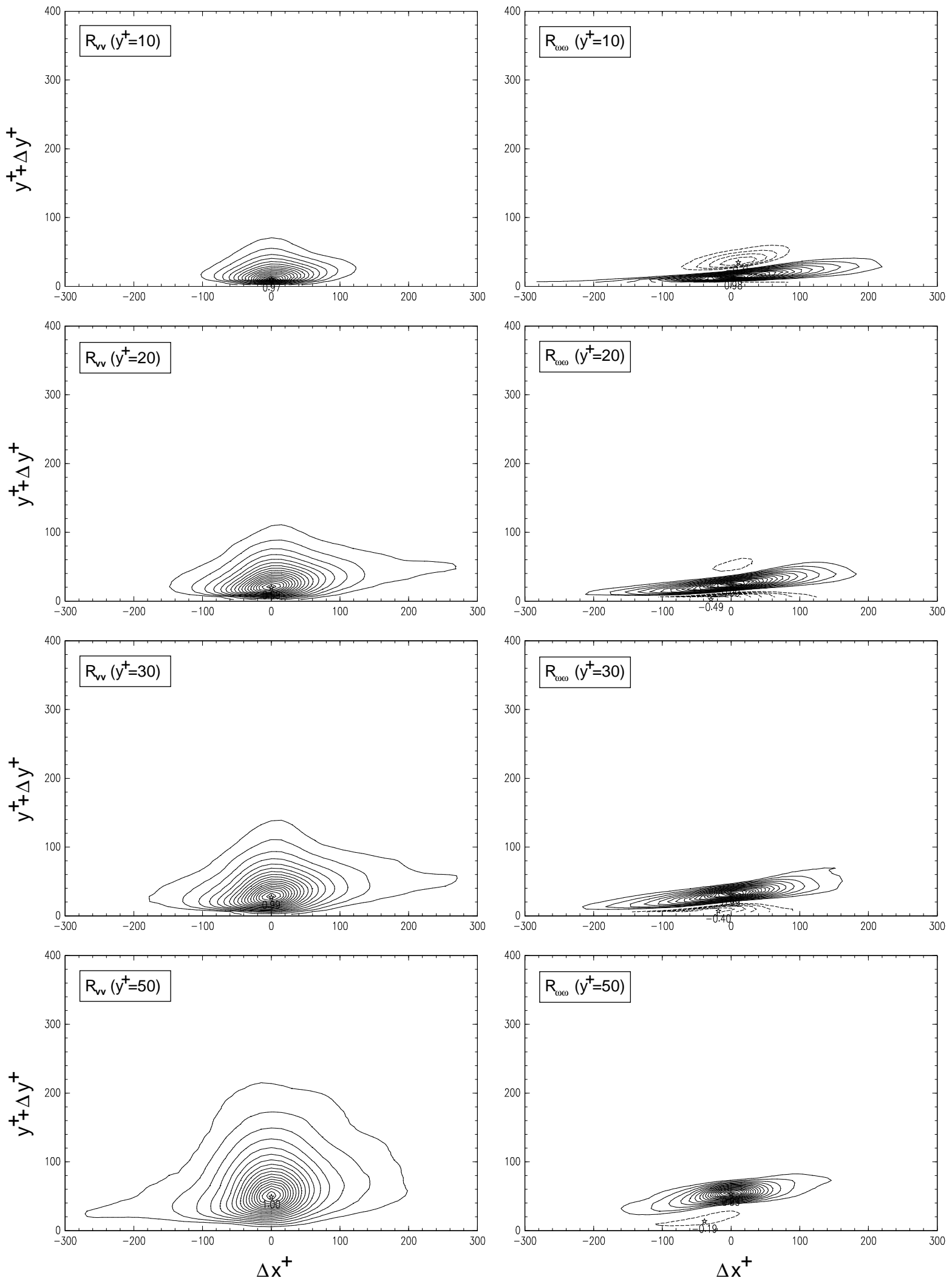

FigURE 5.11: $R_{v v}\left(\Delta x^{+}, y^{+}, y^{+}+\Delta y^{+}\right)$(left) and $R_{\omega_{z} \omega_{z}}\left(\Delta x^{+}, y^{+}, y^{+}+\Delta y^{+}\right)$(right) for various $y^{+}$-locations of the fixed point (see legend) measured at $R e_{\theta}=7800$. 
to a large extent. This illustrates clearly the difficulty associated with the interpretation of the one-dimensional correlations. Nevertheless, this representation allows a convenient comparison of the various correlations and their dependence on the location of the fixed point relative to the wall. Especially the different size of the structures along the coordinates is clearly visible (note different scale). Figure 5.11 reveals the two-dimensional correlation function of the wall-normal velocity fluctuations $R_{v v}$ and the correlation function of the span-wise vorticity component $R_{\omega_{z} \omega_{z}}$ for different locations of the fixed point. It can be clearly seen that the $R_{v v}$ correlations are relatively small with respect to $R_{u u}$ and also strongly deformed for wall locations below $y^{+}=100$. For $y^{+}>100$, this deformation vanishes as can be seen from the line representations in figure 5.9 and figure 5.10. Remarkable is the extension of the overhang region which goes in stream-wise direction when the fixed point is below 40 wall-units and in up-stream direction for larger wall-distances. The vorticity structures shown
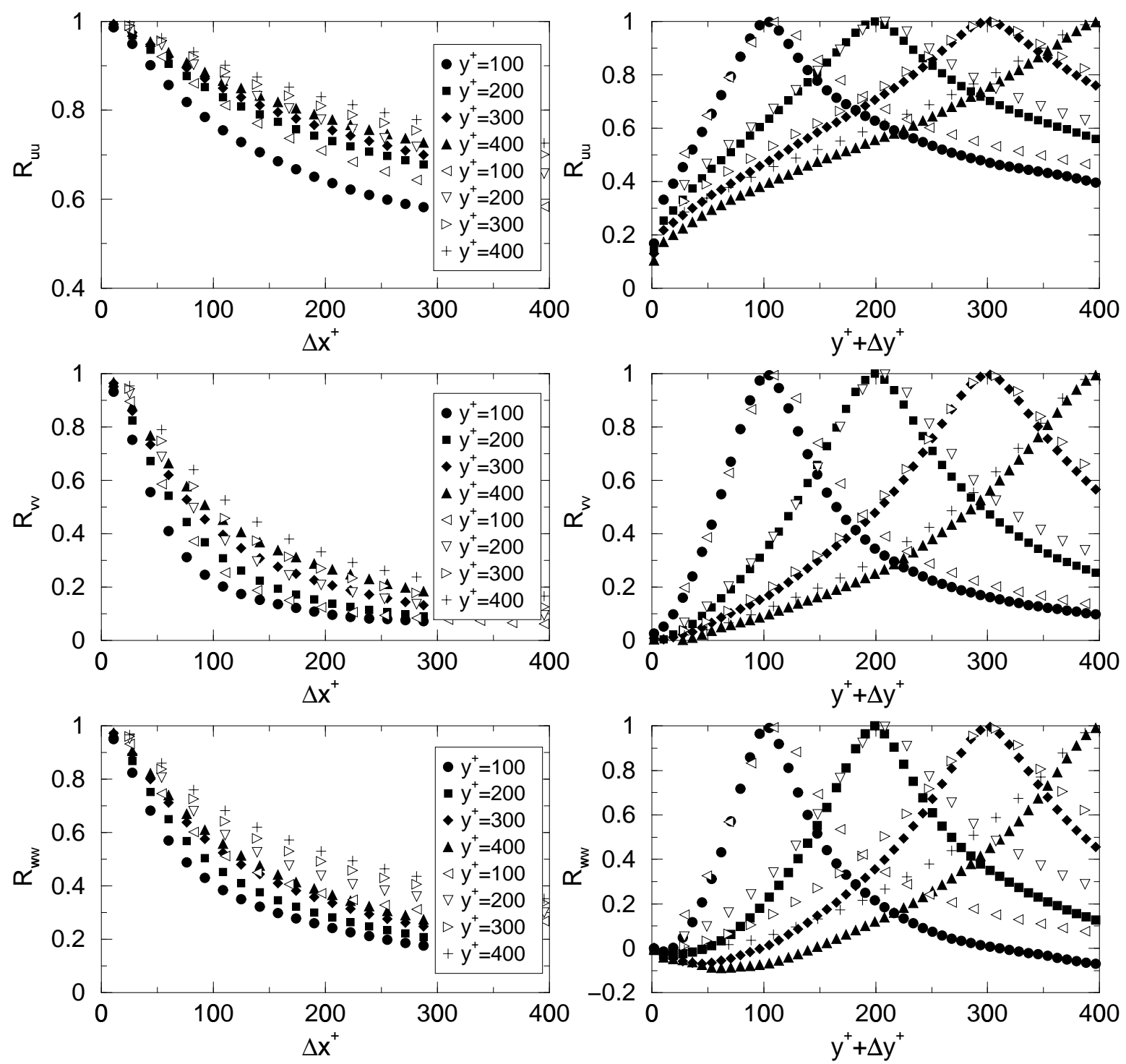

FIgURE 5.12: Comparison of $R_{u u}\left(\Delta x^{+}, y^{+}, y^{+}+\Delta y^{+}\right), \quad R_{v v}\left(\Delta x^{+}, y^{+}, y^{+}+\Delta y^{+}\right)$and $R_{w w}\left(\Delta x^{+}, y^{+}, y^{+}+\Delta y^{+}\right.$) measured at $R e_{\theta}=7800$ (solid symbols) and $R e_{\theta}=15000$ (opaque symbols) and $y^{+}=100,200,300,400$. 
in the right column of figure 5.11 are strongly elongated close to the wall and it can be seen that a remarkable region of negative correlation (dashed line) exists above the region of positive correlation (solid line). For larger wall distances such a region of negative correlation can be observed below the main structure but with reduced intensity. In addition it can be seen that the main structure is slightly inclined relative to the stream-wise coordinate and that the inclination angle increases in stream-wise direction. Figure 5.12 shows a comparison between the investigations performed at $R e_{\theta}=7800$ (solid symbols) and $R e_{\theta}=15000$ (opaque symbols). The left column reveals the stream-wise dependence of the primary correlations measured at $y^{+}=100,200,300,400$ and the right column the corresponding dependence on the location of the fixed point from the wall. Although the general dependence of the correlations agrees fairly well when compared in wall-units, the correlation value of the high
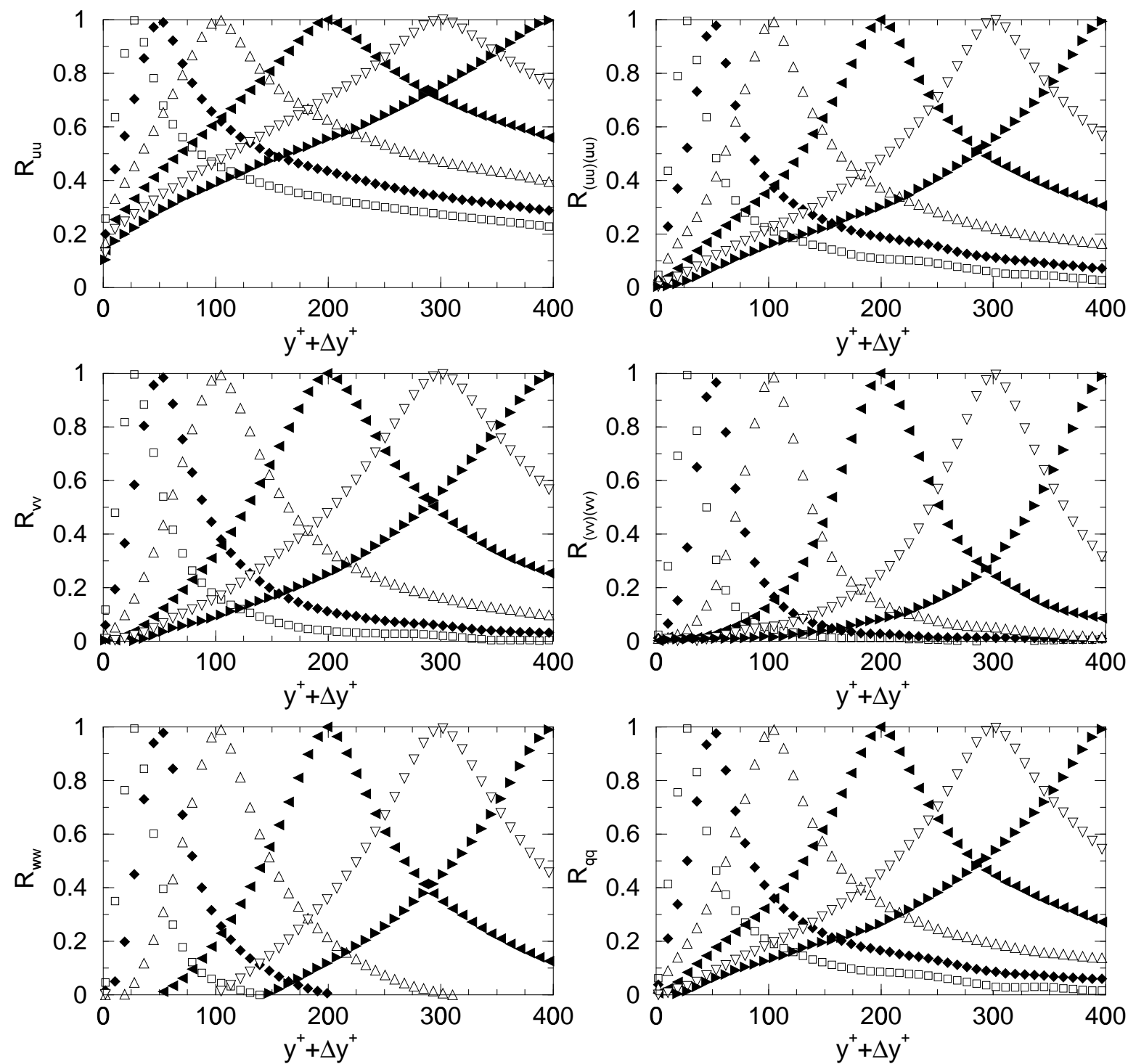

FIGURE 5.13: $R_{u u}\left(\Delta x^{+}, y^{+}, y^{+}+\Delta y^{+}\right), R_{v v}\left(\Delta x^{+}, y^{+}, y^{+}+\Delta y^{+}\right)$and $R_{w w}\left(\Delta x^{+}, y^{+}, y^{+}+\Delta y^{+}\right)$ (left), $R_{(u u)(u u)}\left(\Delta x^{+}, y^{+}, y^{+}+\Delta y^{+}\right), R_{(v v)(v v)}\left(\Delta x^{+}, y^{+}, y^{+}+\Delta y^{+}\right)$and $R_{q q}\left(\Delta x^{+}, y^{+}, y^{+}+\Delta y^{+}\right)$ (right) measured at $R e_{\theta}=7800$. The maximum of correlation indicates the position of the fixed point (same symbols as in figure 5.9). 
Reynolds number investigation is sometimes up to $10 \%$ above the values of the low Reynolds number measurements. Furthermore it should be noted that the largest difference appears for the $R_{w w}$ correlation. However, no unique statement can be given whether this is a real Reynolds number effect or a result of the tilted nature of the correlations, because in case of non-isotropic turbulence it is not well defined how the correlations must be compared when the Reynolds number varies (it is conceivable that only the main axis of the ellipse collapses). Figure 5.13 and figure 5.14 finally display a line representation of the various double- and triple-correlations, which are associated with the production of turbulence according to equation (5.18) and (5.20), namely $R_{(u u)(u u)}, R_{(v v)(v v)}, R_{q q},-R_{u v},-R_{v u}, R_{(u v)(u v)}, R_{(u u v)(u u v)}$, $R_{(u v v)(u v v)}$ and $R_{(v w w)(v w w)}$ measured at $R e_{\theta}=7800$. It can be clearly seen that the width of the correlations at a particular value decreases in general with increasing complexity of the correlations $R_{u u}>R_{(u u)(u u)}>R_{(u u v)(u u v)}$. This implies a decreasing importance of the higher order correlations and establishes the simplifications usually applied in the formulation
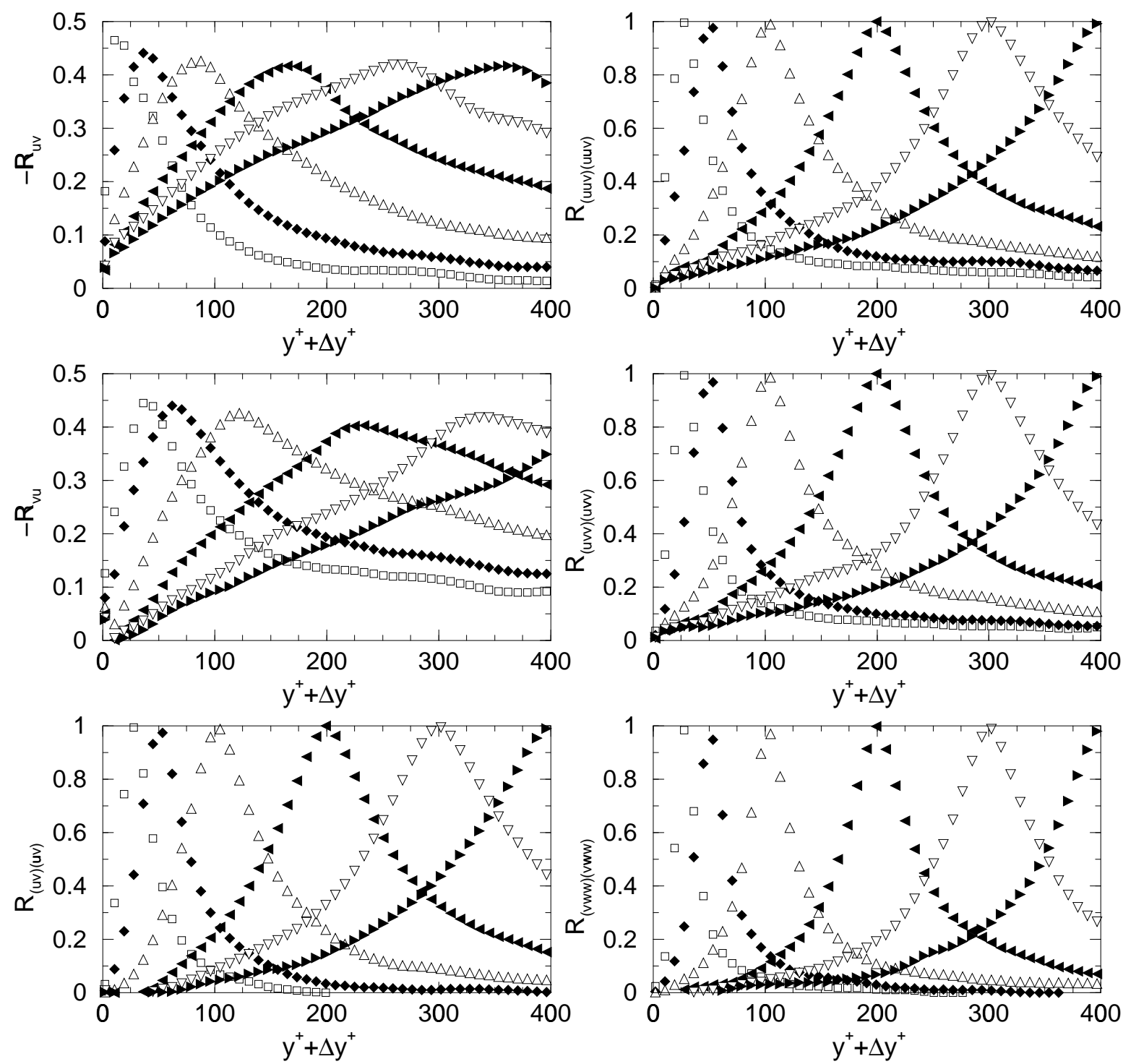

FIGURE 5.14: $R_{u v}, R_{v u}$ and $R_{(u v)(u v)}$ correlations (left) and $R_{(u u v)(u u v)}, R_{(u v v)(u v v)}$ and $R_{(v w w)(v w w)}$ (right) measured at $y^{+}=20,30,50,100,200,300,400$ and $R e_{\theta}=7800$. 
of conservation equations for the Reynolds stresses [37, 89]. Of primary interest are the $-R_{u v}$ and $-R_{v u}$ correlations and their dependence on the wall distance, because these functions are mainly responsible for the turbulent mixing in wall-bounded flows according to the Reynolds equation in the boundary layer approximation on page 70 . For the interpretation it is important to keep in mind that the first subscript indicates the velocity component at the fixed point while the second one indicates the component which is spatially shifted for the calculation of the cross-correlation. There are two surprising results, namely the different height of the correlation in the vicinity of the wall and the shift of the maximum towards the wall (when the stream-wise velocity component is fixed) or away from the solid boundary (when the wallnormal velocity component is fixed). This result will be further examined in chapter 6 .

\subsection{Properties of coherent velocity structures}

The multi-point correlations presented in the previous section furnish information about the average size, shape and coherence of the flow structures being present in the flow field, and they provide valuable details required for the interpretation of near-wall turbulence in terms of Reynolds equation and multi-point correlation equations. However, as the flow structures have a history of development while they are transported downstream, the spatial correlation functions include realizations of a large number of structures at various stages of their life history. As the details about the individual flow structures are averaged out by the statistical approach, the organisation of the individual coherent flow structures, their significance for the production of turbulence and their relation with respect to proposed vortex models will be investigated in the following. Unfortunately, this is not a trivial task because the selection of typical structures is subjective as the outcome of such an approach is always strongly coupled with the specified criteria. In other words, it is always possible to find structures which nicely match with the models proposed in the first chapter or structures with other desired properties. In order to minimise this well known problem, several techniques have been successfully exploited in the past, such as the variable interval time averaging (VITA) method, which compares the short term variance of a flow variable with respect to a chosen threshold level, or the variable interval space averaging (VISA) method, which allows to detect structures with similar spatial structure. However, these methods, which were mainly developed for the analysis of time-resolved hot-wire measurements, are not well suited for the analysis of PIV velocity fields. Therefore flow structures will be investigated here which appear frequently and with sufficient strength in the essential Reynolds stress component. The first criterion selects structures which are dominant with regard to their occurrence and the second one identifies structures which are dominant in terms of turbulent mixing and production of turbulence.

\subsubsection{Shear-layer}

The basic coherent flow structure that can be easily observed in the $x y$-plane of a turbulent boundary layer (stream-wise wall-normal) is the so called shear-layer. When moving with the appropriate convection velocity, or after subtracting the local mean velocity, this structure appears as a slightly-inclined low-speed region, often several thousand wall-units in length and some 100 wall-units in height (their width is largely unknown). The inclination angle of the shear-layers relative to the wall was the subject of several studies $[9,28,50,62,80]$. The general consensus is that this angle depends on the distance from the wall, as indicated by the 
results in figure 5.5 and 5.6, but the exact values are still a point of discussion. The results, based on the analysis of space-time correlation measurements, vary between 4 and 7 degrees in the viscous sub-layer and 11 and 20 degrees in the buffer region [63]. This observation is fully consistent with the average results presented in section 5.3.2. On the top of these layers, vortices with a strong span-wise vorticity component can be observed frequently [74, 38], but it is an open question whether these vortices are a result of a Kelvin-Helmholtz instability, like
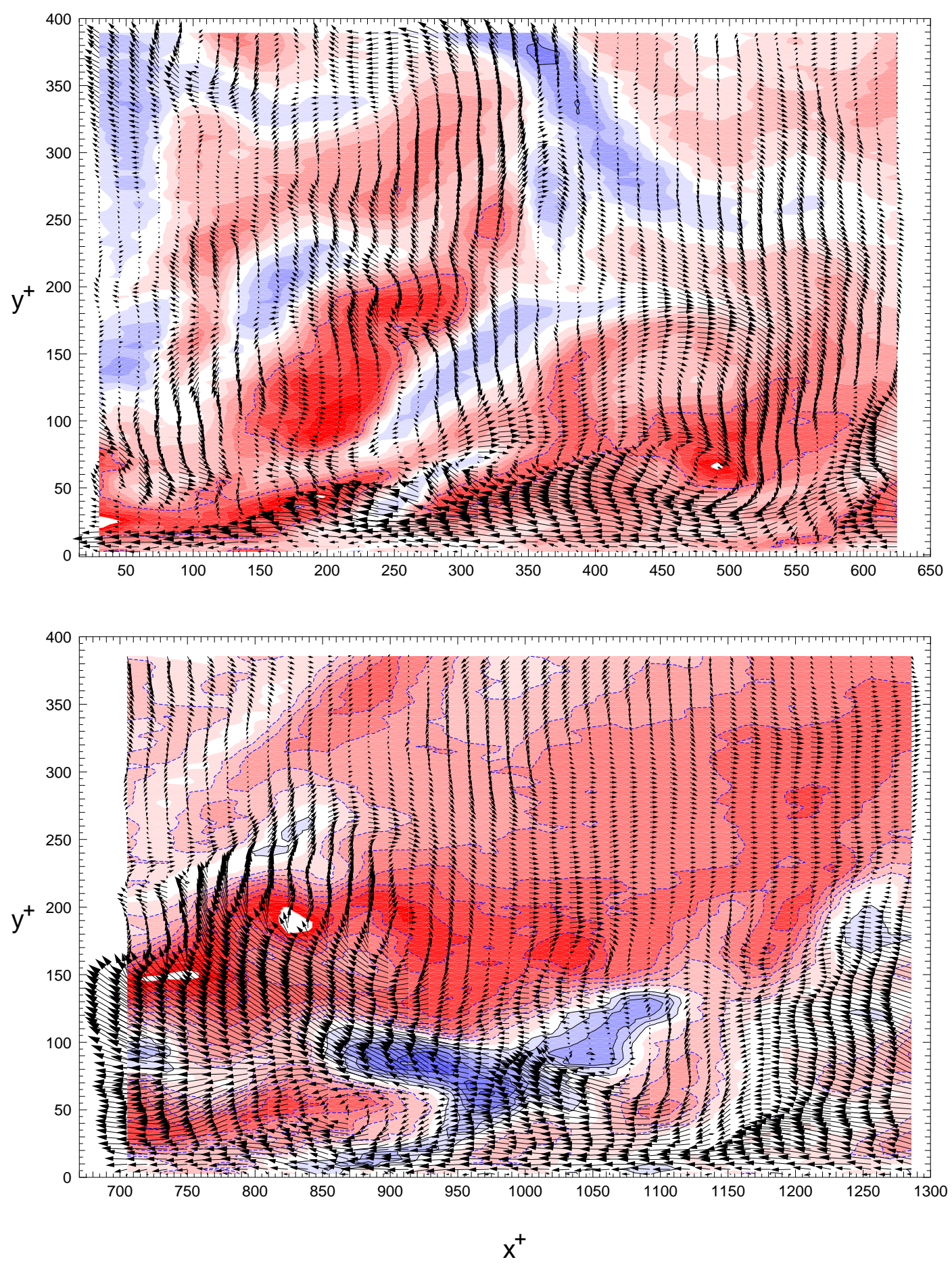

FIGURE 5.15: Velocity fluctuations measured at $\operatorname{Re}_{\theta}=7800$ ( $w$ is colour coded). 


\section{Investigation of the $x y-p l a n e$}

spiralling Kelvin-Helmholtz vortices in a mixing layer, or related to the heads of a family of loop-shaped structures as assumed by many authors. Figure 5.15 shows a typical pair of instantaneous velocity fields measured simultaneously with the double-stereoscopic PIV system $18 \mathrm{~m}$ behind the leading edge of the flat plat at $R e_{\theta}=7800$ (the measurement domain of the lower vector field is located down-stream of the upper one, as indicated by the $x$-scale). The mean flow direction is from left to right and the local mean velocity is subtracted to display the
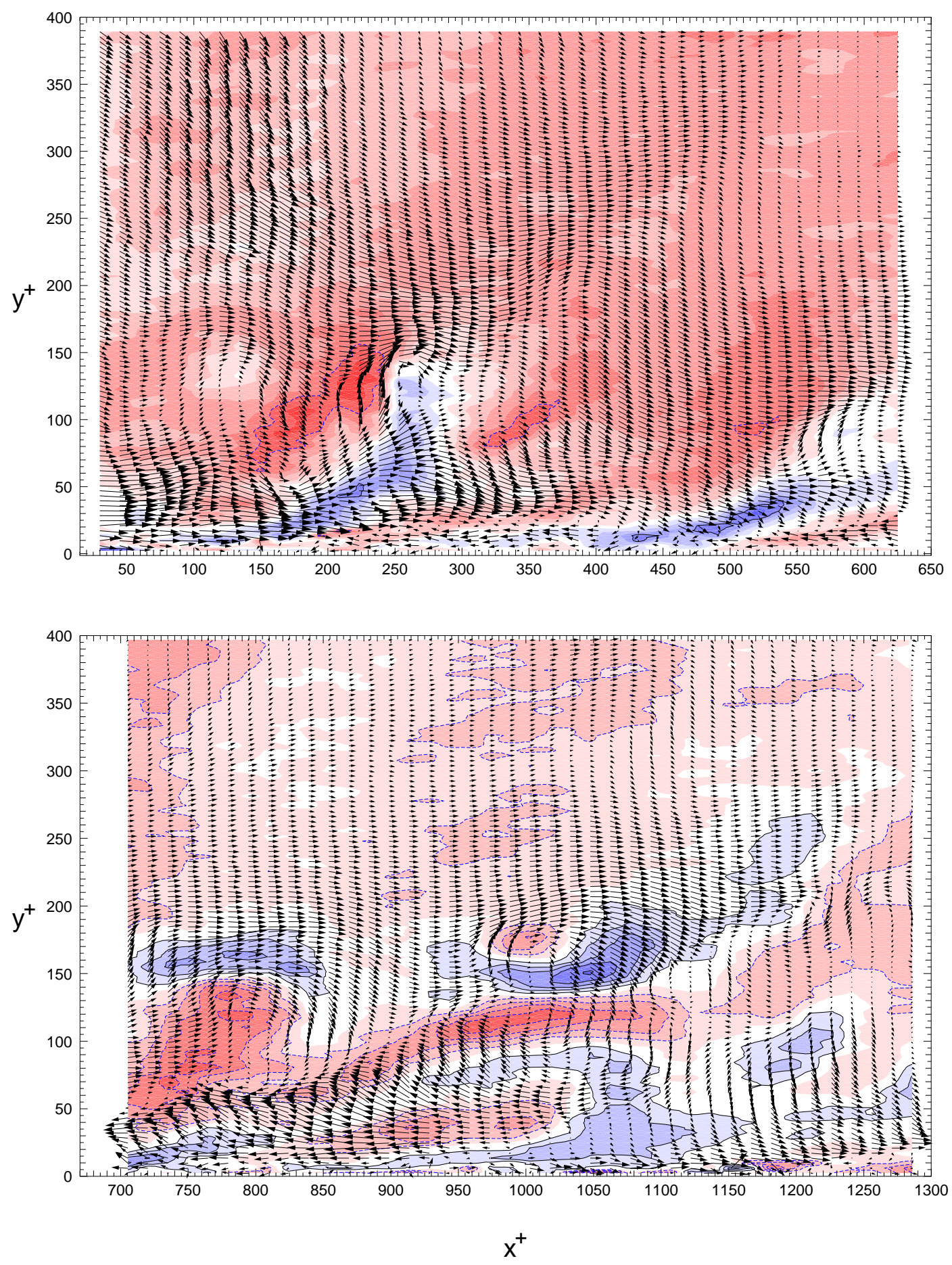

FIGURE 5.16: Velocity fluctuations measured at $\operatorname{Re}_{\theta}=7800$ ( $w$ is colour coded). 
turbulent velocity fluctuations $u(x, y), v(x, y)$ and $w(x, y)$. In this representation, the shearlayer appears as slightly-inclined low-speed regions with the geometrical dimensions given above. Although the exact angle varies strongly in the instantaneous velocity fields, it can be clearly seen that the angle between the wall and the shear-layer increases with increasing wall distance, see also figure 5.16. This can be explained by the different shape of the low momentum structures due to the strong flow gradients and the presence of the wall. While the near-wall region below $y^{+}=50$ is dominated by low-speed streaks, which will be considered in the next chapter in detail, the log-law region above $y^{+}=50$ is dominated by large-scale shear-layers without any obvious structural regularity in contrast to the streaks. Another remarkable result is the fact that the low momentum structures below $y^{+}=50$ are usually not as long as the total length of the large-scale shear-layer. However, as the low-speed streaks are only 30 wall-units in width and not often perfectly parallel to the main flow direction, as will be seen later, only a cut through the streak can be observed in the $x y$-plane while the length of the cut depends on the orientation of the streak with respect to the measurement plane and its geometrical properties. In figure 5.16 for example, a cross-section of a streak can be observed from $x^{+}=150$ to 400,500 to 800 and another starts at $x^{+}=1200$. The lower vector field of the same figure and the upper one of figure 5.15 indicate that on the top of these shear-layers vortices can be frequently observed. The significance of these vortices for the turbulent mixing will be investigated in the following.

\subsubsection{Ejection}

Since the pioneering near-wall flow visualisation experiments by Kline et al. (1967), Corino \& Brodkey (1969) and Kim et al. (1971), it is well established that the coherent structures, which can be identified in the flow field, run through quite complex cycles. The most striking cycle or process, which could be observed, was the movement of elongated low velocity regions, designated as low-speed streaks, out in the buffer region. This motion away from the wall was associated with an oscillation of the low-speed flow structure and a violent break-up into small scale turbulence. A schematic representation of this process along with the typical velocity profiles is shown in figure 1.2 on page 6 . This near-wall process, which was observed to occur suddenly and abruptly, was designated as ejection and it was speculated that a large part of the turbulence production was associated with this observation. This becomes evident from the production term shown in the following equation. Due to the fact that the mean velocity gradient $\partial \bar{U} / \partial y$ is quite large close to the wall, according to figure 5.2, and the product of the two orthogonal velocity fluctuations $u$ and $v$ is negative, because the stream-wise velocity fluctuation $u$ is negative for low-speed streaks and their wall-normal velocity component positive according to the observations, the total term becomes positive when multiplied with the negative sign. This means that turbulence is produced according to equation (5.18).

$$
-\rho \overline{u v} \frac{\partial \bar{U}}{\partial y}=\text { turbulence production }
$$

Based on these considerations quantitative experiments were performed to determine the footprint of this ejection process in detail. It was found that the typical velocity signal from the process was quite regular provided the scales and intensity were normalised properly (an example of the signal is shown on page 133). Figure 5.17 reveals two characteristic veloc-

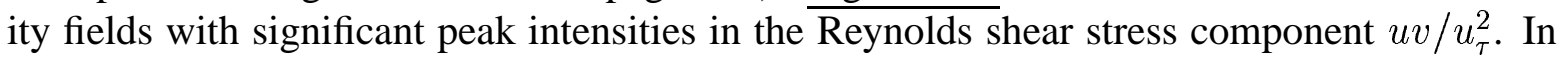


case of the upper vector field the range is $-20.0 \leq u v / u_{\tau}^{2} \leq 7.2$ and for the lower image $-26.2 \leq u v / u_{\tau}^{2} \leq 6.7$. In both cases the region where the production of turbulence takes place (red colour) is embedded in the typical low-speed regions close to the wall, and it should be noted that on top of the low-speed region a vortex can be observed, as already mentioned at the end of the previous section. It is evident that these vortices pump low-speed fluid away
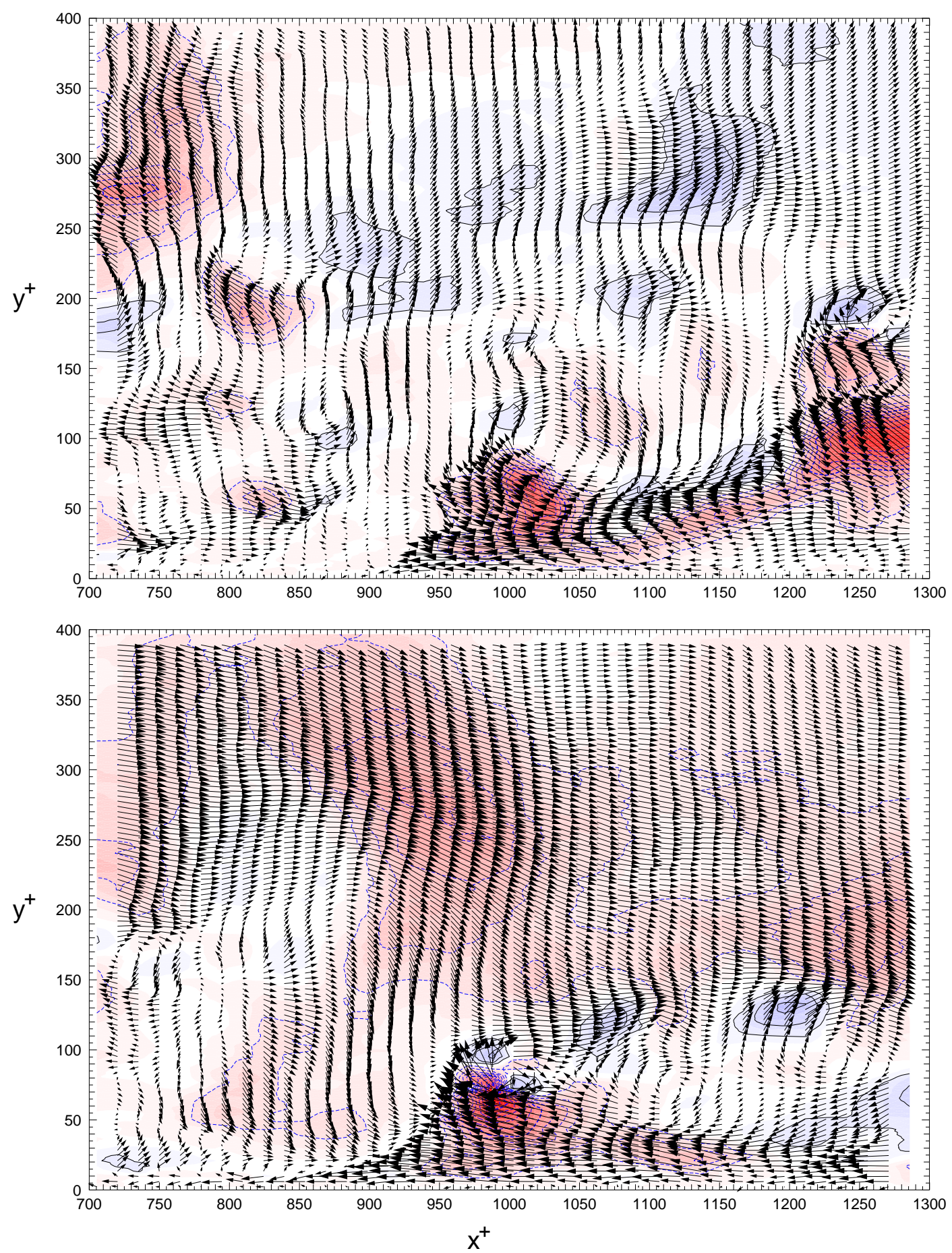

FIGURE 5.17: In-plane velocity fluctuations and distribution of the instantaneous Reynolds shear-stress (red: $u v<0$, blue: $u v>0$ ) measured at $R e_{\theta}=7800$. 
from the wall and this is associated with the production of turbulence because $u v$ is negative. In addition it becomes visible that upstream of the low-speed region a well developed shearlayer appears. Compared to the previously discussed shear-layer angle, here the angle is large in relation to the wall.

\subsubsection{Sweep}

In this section the interaction between the shear-layer and the near-wall structures with the surrounding high momentum flow structures coming from larger wall distances under conservation of their momentum will be investigated. The extended high momentum flow structures moving towards the wall are called sweeps in the literature, see page 71 . Figure 5.18 shows two examples which were selected due to the strong intensity in the Reynolds shearstresses component $u v$ that is approximately 20 times larger than the average value shown in figure 5.4. In case of the top vector field, for example, the non-dimensional range is $-22.2 \leq u v / u_{\tau}^{2} \leq 12.2$ and for the lower figure $-23.6 \leq u v / u_{\tau}^{2} \leq 18.4$. It should be mentioned that nearly no measured velocity field indicates a higher value which means that the structures can be considered as dominant concerning the production of turbulence. However, these velocity field represents general features which can be frequently observed even when the peak intensity of the Reynolds shear stress is lower. It can be seen from both velocity fields that sweeps are relatively simple large-scale $e d d y$ structures which transfer high momentum fluid towards the wall as assumed in the mixing-length theory by Prandtl. The word eddy implies a local flow region (or structure) over which the simultaneous velocity fluctuations are coherent or correlated $[89,100]$ and not a circulatory motion. As the variation of the stream-wise velocity inside the sweep is relatively small, the significance of this flow structure with regard to the production to turbulence is basically determined by the velocity fluctuation in wall-normal direction. This becomes important in case of boundary layer control for example, because a velocity disturbance in wall-normal direction is much more efficient than a disturbance in stream-wise direction. However, as a large part of the sweeps is located farther away from the wall, the potential artifical disturbance must be intense enough to reach the flow structure.

The results presented in this chapter clearly indicate the existence of coherent structures, their geometrical properties, and their significance for the turbulent mixing in wall-bounded flows. In the following chapters the interaction of these structures will be further examined in order to find out which structures are fundamental and which ones are only secondary, which ones are dominant and which ones are irrelevant. 

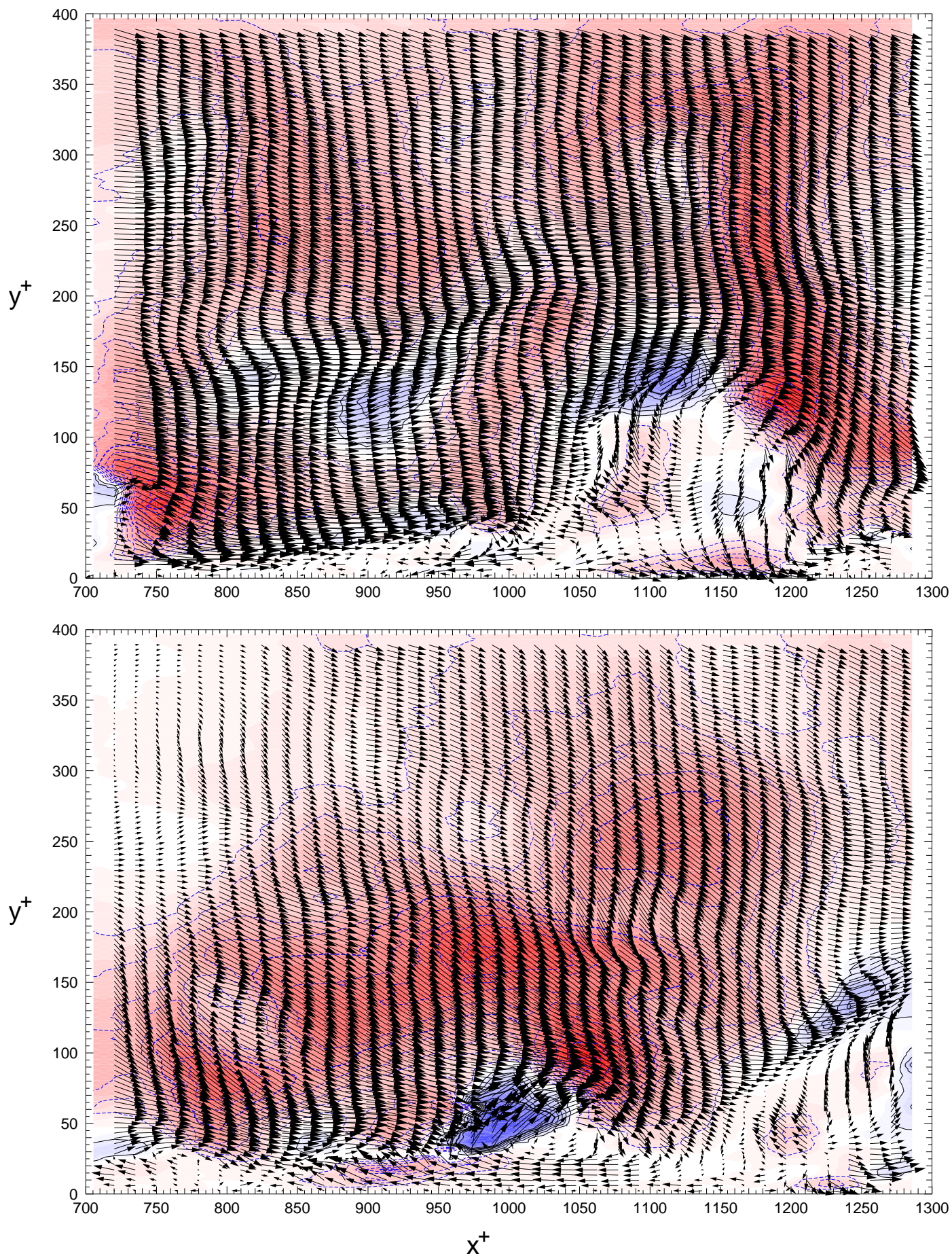

FIGURE 5.18: In-plane velocity fluctuations and distribution of the instantaneous Reynolds shear-stress (red: $u v<0$, blue: $u v>0$ ) measured at $R e_{\theta}=7800$. 


\section{Investigation of the xz-plane}

One of the well established and widely accepted results of the turbulent boundary layer research is the characteristic mean velocity profile and the maximum of the stream-wise velocity fluctuation in the buffer layer at $y^{+} \approx 15$, see $[21,55]$. This is shown in the lower left graph of figure 6.1 measured at $R e_{\theta}=7800$ with the stereoscopic PIV arrangement described in chapter 5. However, when the stream-wise fluctuations are subdivided according to their sign before calculating the rms values, it turns out that the negative fluctuations have a maximum at $y^{+} \approx 20$, while those with a positive component reach the maximum at $y^{+} \approx 10$, com-
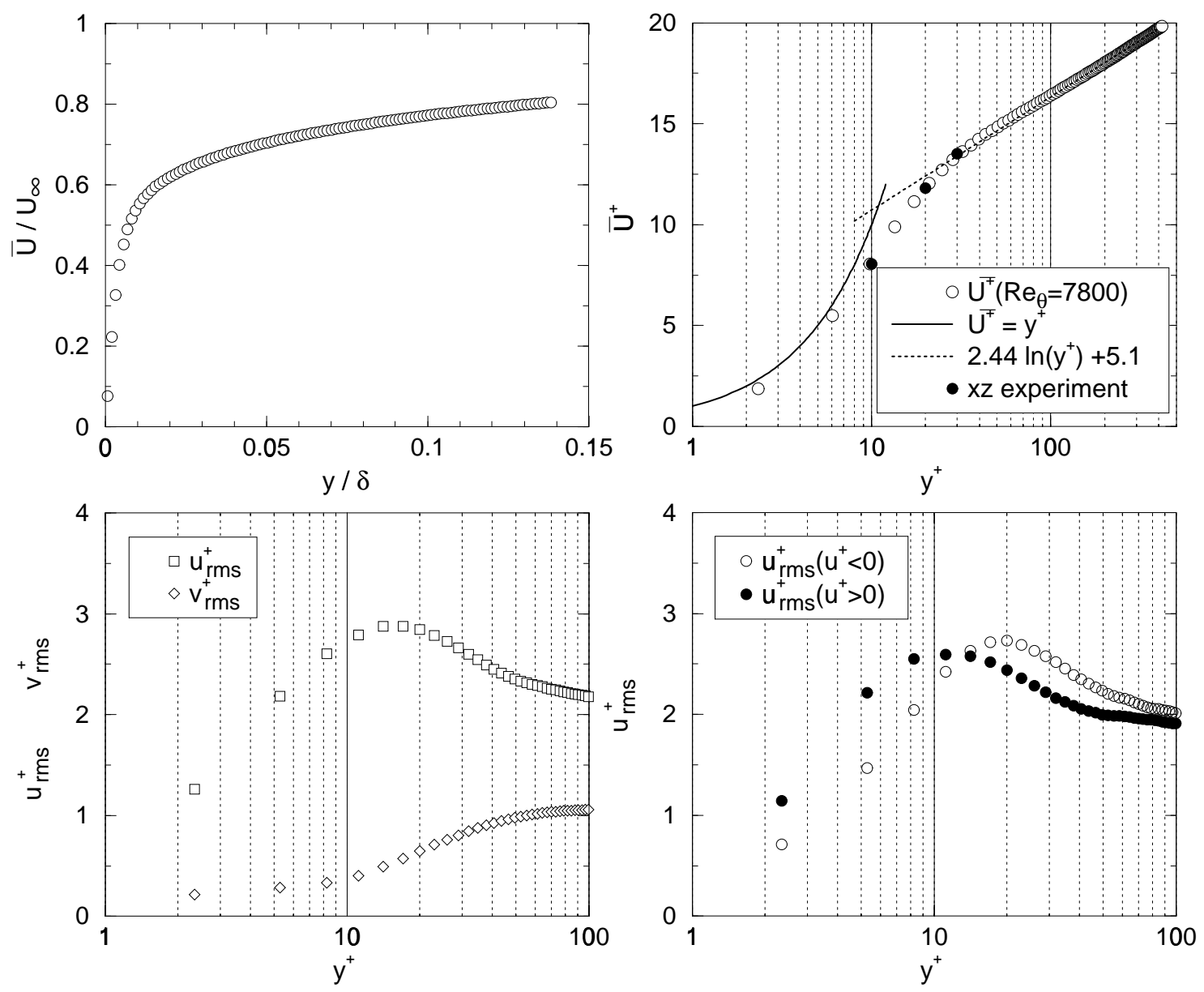

FIGURE 6.1: Results of high-resolution stereoscopic PIV boundary layer measurements performed at $R e_{\theta}=7800$. Top: Linear and semi-logarithmic representations of the normalised mean velocity profile (average over 2500 velocity fields). Bottom: Root-mean-square values of the stream-wise velocity fluctuations $u$ (left) and conditional averaged representation (right). 
pare [111]. This is shown in the lower right graph. It is not surprising that the maxima do not coincide as the high momentum flow structure originates statistically from $y^{+}$locations where $\bar{U}^{+} \sim \ln y^{+}$while low-momentum flow structures have their origin in the near-wall region on average, where $\bar{U}^{+} \sim y^{+}$holds (see upper right figure). However, the reason that the maximum of $\sqrt{u^{2}}$, with $u<0$, is further away from the wall with respect to $\sqrt{\overline{u^{2}}}$, with $u>0$, is not evident. To examine the physical process associated with this experimental result, the statistical properties of a fully developed turbulent boundary layer flow along a flat plate is investigated in stream-wise span-wise planes located at $y^{+} \approx 10,20$ and 30 , by using the multiplane stereo PIV technique described in chapter 4 . First of all, the joint probability density function of the velocity fluctuations is analysed to determine the basic statistical properties of the coherent velocity structures like their occurrence, intensity and main flow direction relative to the wall. Thereafter various spatial correlation, cross-correlation and conditional-correlation functions are presented in order to compare the mean size and shape of the dominant flow structures being present in the near-wall region of the flow. The dynamic of the dominant structures is investigated by means of spatio-temporal correlation and cross-correlation functions measured in spatially separated planes. Finally, characteristic flow fields are considered to illuminate the relation between the instantaneous flow structures with respect to the averaged ones and to estimate the contribution of the identified coherent flow structures to the production of turbulence and transport of Reynolds stresses.

\subsection{Experimental set-up}

The experimental investigation was performed $18 \mathrm{~m}$ behind the leading edge of the flat plate in the temperature stabilised closed-circuit wind-tunnel shown in figure 1.4 on page 8 . The multiplane stereo PIV-equipment applied for the investigation consists of the same BMI four pulsed Nd:YAG laser system as outlined in section 5.2, but in contrast to the configuration described on page 72 , here the system was arranged in such a way that the differently polarised light pulses leave the laser housing at different output ports. This allows to cover a wider range of light-sheet spacings (up to a few $\mathrm{cm}$ ) and an independent positioning of both beampairs, as pointed out in section 4.2. Behind each port one optical bench was installed with three appropriate lenses and a $\lambda / 2$-retardation plate which allows to rotate the direction of the polarisation vector continuously. By using long focal length lenses, the laser beams could be formed into sheets of about $0.6 \mathrm{~mm}$ thickness at the measurement position, corresponding to $\Delta y^{+} \approx 5$. It should be noted that the main problem associated with the generation of a lightsheet running parallel to a flat surface is the loss of energy with decreasing wall distance, due to the darkening of the light-sheet by the wall. This difficulty can be solved either by using more optical elements, as described in section 4.8, or by using long focal length lenses (in the meter range) as applied here. The positions of the light-sheets could be smoothly adjusted in vertical direction by moving properly coated mirrors, mounted on a micrometer translation stage. The determination of the distance of the light-sheets from the wall was achieved by illuminating a square metal block, placed on the flat plate and covered with light-sensitive paper. The recording system utilised for this investigation is similar to the unit described in section 5.2 apart from the lenses, which where replaced by $100 \mathrm{~mm}$ Carl Zeiss lenses because of the short observation distance $d_{o}$ (see table 6.1), and the required polarising beam splitter-cubes and mirrors in front of the lenses according to section 4.1. The arrangement was installed below the wind-tunnel, as shown in figure 6.2, at positions summarised in table 6.1. The positions of 
the (virtual) slave cameras 1 and 2, located behind the mirrors, are not presented in the table as these can be considered as identical with the corresponding master cameras 3 and 4 .

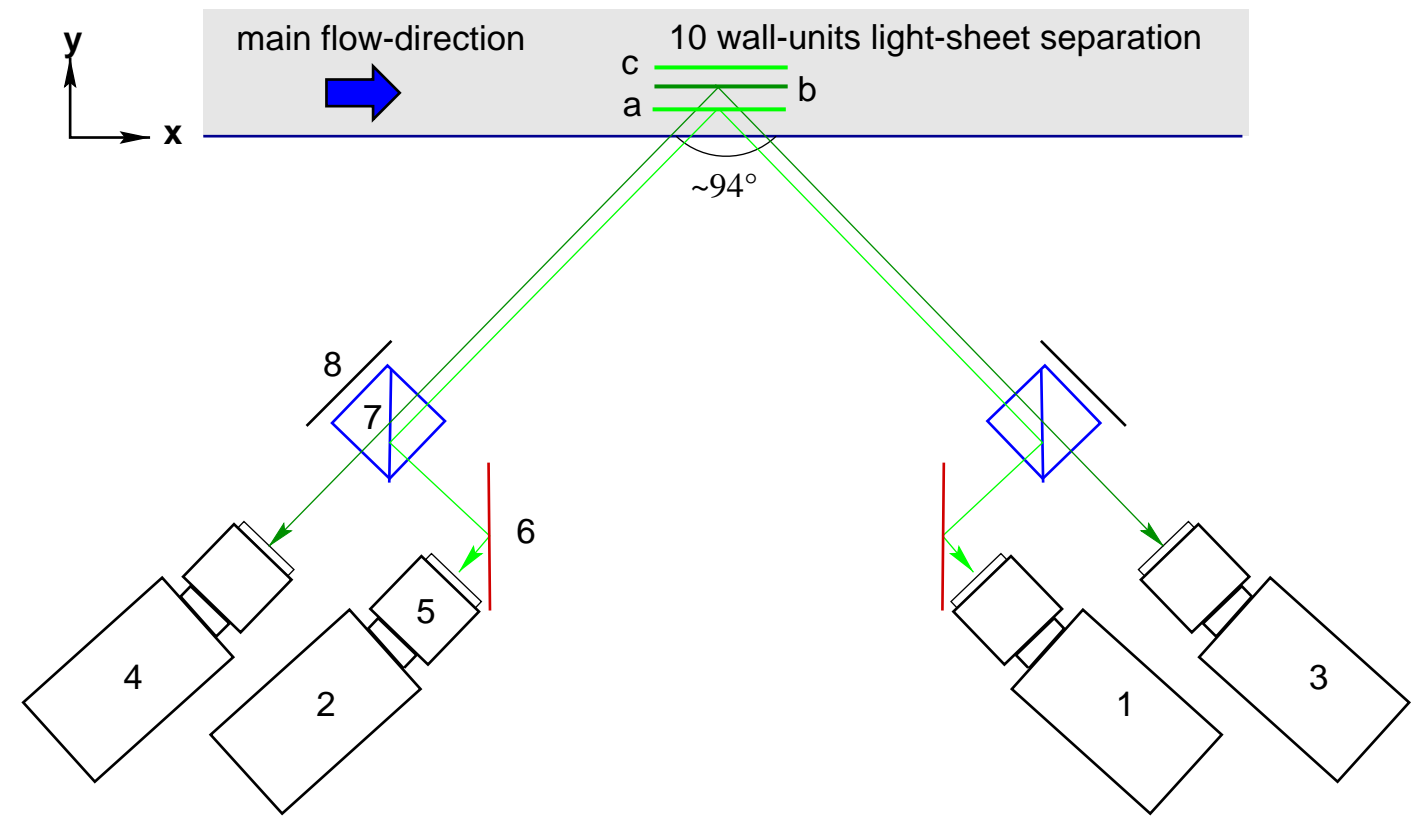

FIGURE 6.2: Experimental set-up for $x z$-investigation. 1-4 digital cameras, 5 lens, 6 mirror, 7 polarising beam-splitter cube, 8 absorbing material.

\begin{tabular}{ccccc}
\hline camera & $x[\mathrm{~mm}]$ & $y[\mathrm{~mm}]$ & $d_{o}[\mathrm{~mm}]$ & $\alpha[\mathrm{deg}]$ \\
\hline 3 & 628 & -573 & 850 & 47.1 \\
4 & -608 & -565 & 829 & 47.6 \\
\hline
\end{tabular}

TABLE 6.1: Camera position, observation distances and viewing angles with respect to the centre of the field of view.

For magnification and field of view adjustments each Scheimpflug-adapter was mounted on a two-axis micrometer translation stage, and the polarising beam splitter-cubes and mirrors in front of the lenses were connected to two-axis tilt-rotation stages and gimbal mirror mounts. To obtain ideal particle images for the image analysis algorithms (bright circles, $2-3$ pixel in diameter, surrounded by a dark background), the imaging of the field of view was performed by means of $100 \mathrm{~mm}$ Carl Zeiss lenses with an aperture of 8 . This leads to a complete erasure of all optical aberrations and out-of-focus effects. The arrangement was installed below the wind-tunnel, as shown in figure 6.2. The mean observation distance was $840 \mathrm{~mm}$ and the opening angle between the left and right camera systems was set to $94.4^{\circ}$ to resolve the out-ofplane motion with sufficient accuracy according to figure 3.5. This is important because the out-of-plane component is required to calculate the dominant Reynolds shear-stress component $\tau_{\text {turb }}=-\rho \overline{u v}$. For the calibration of the system a regular grid with $2 \mathrm{~mm}$ line spacing was glued on an aluminium plate and attached with a micrometer translation stage in such a way that a parallel motion of the grid could be achieved in vertical direction. This grid was aligned with each light-sheet one after another and recorded each time with the four cameras (before and after the experiment in order to proof the conservation of the boundary conditions during the experiment). As any horizontal translation of the target could be excluded with this device, 
a particular $x z$-position in a plane at $y$ can be precisely related to a $x z$-position in a plane at $y+\Delta y$. This is extremely important because any uncontrolled movement of the calibration target would appear in the correlation data of the velocity fluctuations and could bias the interpretations of the results, as pointed out in section 3.3. Due to the small spatial separation of only ten wall-units between the differently polarised light-sheet planes, it was not necessary to move any camera when changing the measurement position, as all effects could be uniquely determined by the calibration technique, described in section 3.2, along with the calibration validation method described in section 3.3. Only the sharpness was slightly adjusted in order to keep the image contrast and to avoid out-of-focus effects. The determination of the mapping function was achieved by using the Hough transformation algorithm [18] and the evaluation of the stereo-scopic images was performed on the DLR SUN-cluster by applying the properly normalised free-shape correlation described in section 5.2 and [88]. It should be mentioned that window-deformation techniques, which are increasingly applied for the evaluation of PIV recordings, are of minor value when the main flow gradients are perpendicular to the lightsheet plane. This is due to the fact that the variation of the particle image shift inside an interrogation window is in general not a simple function in contrast to the case where the strong flow gradients coincide with the measurement plane. For the displacement estimation with sub-pixel accuracy, a two-dimensional Gaussian peak-fit routine was applied as this approach is less sensitive to peak-locking effects due to the small variation of the measurement error with the sub-pixel displacement, see section 2.4.2. By applying the following set of band-pass and gradient filters $\left(1<\Delta x<12\right.$ pixel; $-4<\Delta y<4$ pixel and $\Delta x_{i}-\Delta x_{i+1}<5$ pixel $)$, the number of correct measurements was on average above $99.9 \%$ and no smoothing algorithm was applied at all. The basic details about the recording and evaluation are summarised in table 6.2.

TABLE 6.2: Relevant parameter for the characterisation of the experiment performed 18 $\mathrm{m}$ behind the leading edge of the flat plate in the $x z$-plane of the turbulent boundary layer flow.

\begin{tabular}{lrl}
\hline$R e_{\theta}$ & 7800 & {$[1]$} \\
$R e_{\delta}$ & 74000 & {$[1]$} \\
$R e_{x}$ & $3.6 \times 10^{6}$ & {$[1]$} \\
\hline$U_{\infty}$ & 3 & {$[\mathrm{~m} / \mathrm{s}]$} \\
$u_{\tau}$ & 0.121 & {$[\mathrm{~m} / \mathrm{s}]$} \\
$\delta$ & 0.37 & {$[\mathrm{~m}]$} \\
$\delta^{+}$ & 3000 & {$[1]$} \\
$t^{+}=t u_{\tau}^{2} / \nu$ & & {$[1]$} \\
field of view & $67 \times 35$ & {$\left[\mathrm{~mm}^{2}\right]$} \\
field of view & $0.18 \times 0.09$ & {$\left[\delta^{2}\right]$} \\
field of view & $544 \times 284$ & {$\left[\Delta x^{+} \Delta z^{+}\right]$} \\
spatial resolution & $1.42 \times 0.6 \times 1.42$ & {$\left[\mathrm{~mm}^{3}\right]$} \\
spatial resolution & $11.5 \times 5.0 \times 11.5$ & {$\left[\Delta x^{+} \Delta y^{+} \Delta z^{+}\right]$} \\
pulse separation $\Delta t$ & 200 & {$[\mu \mathrm{s}]$} \\
dynamic range at $y^{+}=10$ & 1.00 to 9.330 & {$[\mathrm{pixel}]$} \\
dynamic range at $y^{+}=20$ & 1.37 to 11.74 & {$[\mathrm{pixel}]$} \\
dynamic range at $y^{+}=30$ & 2.61 to 11.84 & {$[\mathrm{pixel}]$} \\
\hline vectors per sample & 7936 & \\
number of samples & 4410 & \\
\hline
\end{tabular}




\subsection{Statistical properties of the buffer layer}

\subsubsection{Single point statistics}

It has already been stated that the lower right graph of figure 6.1 implies that the dominant physical processes, which are responsible for the turbulent mixing in near-wall turbulence, change when approaching the wall. This becomes even more evident when the joint probability density function of the velocity fluctuations $u v$ and $u w$ is considered. This can be clearly seen in figure 6.3 for two characteristic wall distances (left column: $y^{+} \approx 10$, right column:

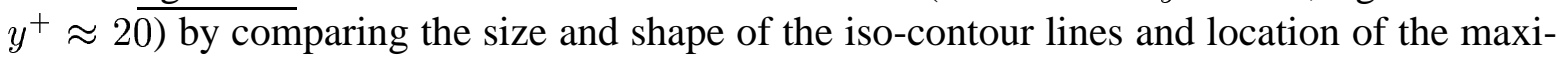
mum. First of all, it is evident that the functions strongly differ in size and shape for a fixed
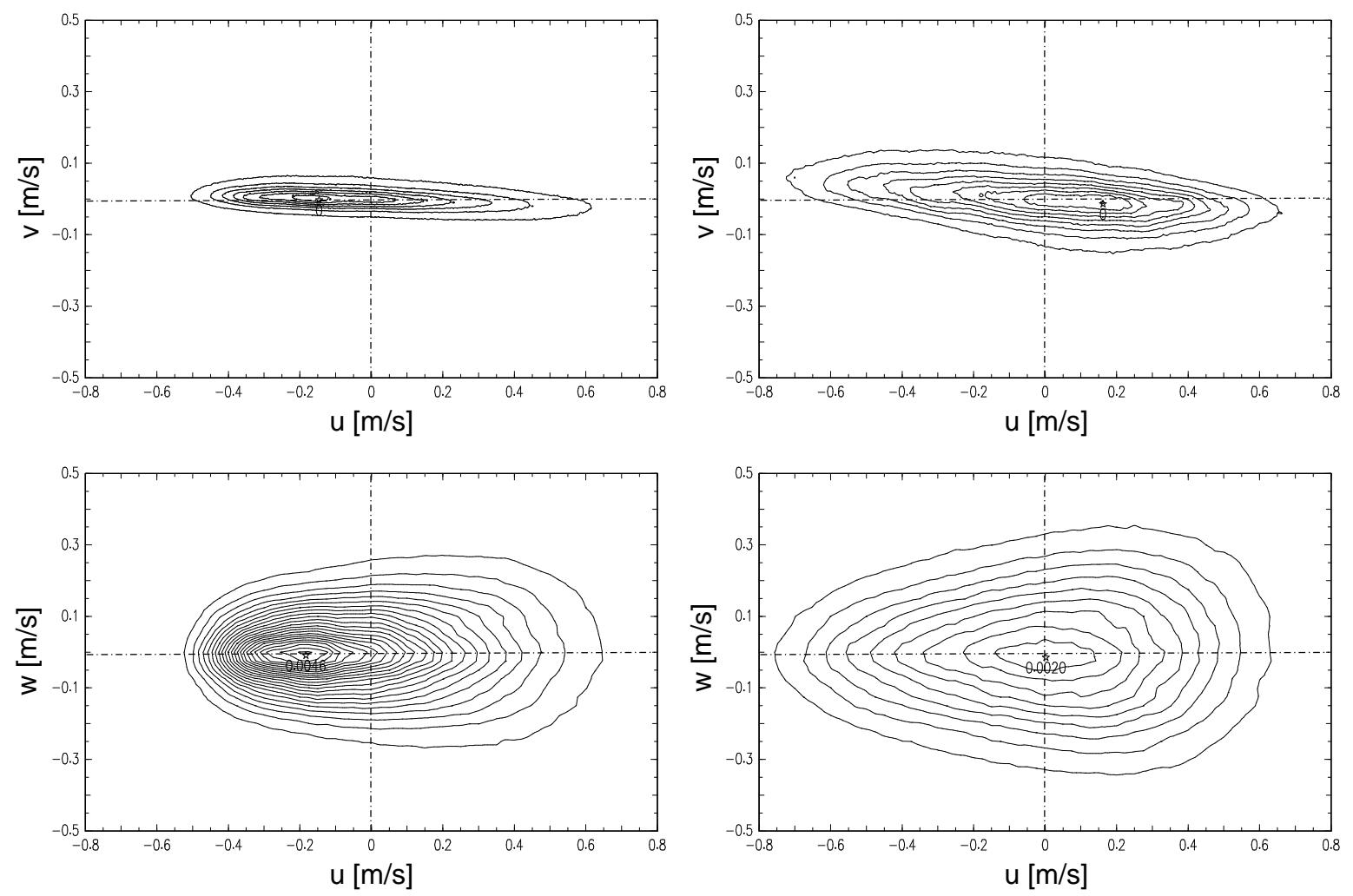

FIGURE 6.3: Joint probability density functions of the $u v$ and $u w$ velocity fluctuations measured at $y^{+} \approx 10$ (left) and $y^{+} \approx 20$ (right).

wall location, because $\sqrt{u^{2}}>\sqrt{w^{2}}>\sqrt{v^{2}}$ according to figure 5.3, and also the symmetry properties $\operatorname{PDF}(w)=\operatorname{PDF}(-w)$ can be explained by the symmetry of the flow in $z$-direction. Secondly, the decreasing size of the distribution towards the wall is evident as the amplitude of the velocity fluctuations is damped due to the presence of the wall. The stretching of the $\operatorname{PDF}(u, v)$ distribution can be explained by the different asymptotic behaviour of the velocity components close to the wall, see [16,31]. Furthermore, the slightly inclined orientation of the $\operatorname{PDF}(u, v)$ function along with the non-symmetrical shape clearly indicates that flow regions associated with ejection $(u<0$ and $v>0)$ and sweeps $(u>0$ and $v<0)$ are more likely and dominate for both wall locations over the regions where both fluctuations possess the same sign simultaneously. This is necessary because the generation and maintenance of turbulence require that $\tau_{\text {turb }}=-\rho \overline{u v}$ is positive on average as can be seen from Reynolds equation in 
boundary layer approximation and the discussion on page 93. However, the displacement of the maximum to negative $u$-values, with decreasing wall distance, is not evident for both PDF's. This implies that at $y^{+} \approx 10$ most of the structures (or the large ones) show a slightly negative stream-wise velocity fluctuation with a rather weak amplitude, as can be seen from the location of the maximum. As the mean value of all fluctuations must be zero by definition, the amplitudes of the structures with a positive stream-wise velocity fluctuation are necessarily larger in order to compensate the maximum at negative $u$. This explains the fact that the maxima at $y^{+} \approx 10$ in the lower right of figure 6.1 result from the positive fluctuations and not from the negative as expected. The power-of-two operation, required for calculating the rmsvalues, increases the weight of the positive large-scale fluctuations when the average is taken and decreases the contribution of the negative high frequency fluctuations (the argumentation can be reversed for the measurements at $y^{+} \approx 20$ ). As the asymptotic behaviour of the spanwise velocity amplitude is weaker with respect to the $v$-component, the displacement of the maximum can be seen more clearly in the lower row of figure 6.3 where the joint probability density function of the velocity fluctuations in stream-wise and span-wise direction is shown. Another interesting feature, appearing in the lower row, is the asymmetry for the large-scale fluctuations, expressed by $\operatorname{PDF}(u, w) \neq \operatorname{PDF}(-u, w)$ which is visible in the right graph. The fact that large-scale fluctuations of the span-wise component $w$ are frequently associated with positive stream-wise fluctuations and small-scale $w$-fluctuations with negative $u$-fluctuations may be an effect associated with organised motions in the flow field. This will be considered later. Figure 6.4 reveals the same result but in a different representation in order to highlight the displacement of the maximum in wall-units and the amplitude of the signal as a function of the parameter indicated in the legend. The graph with the thick line-width in the upper right plot shows the distribution of the wall-normal velocity component when the fluctuation in the stream-wise direction is exactly zero (e.g. the instantaneous velocity component is identical with the time averaged one). The function seems to be symmetrical and Gaussian in shape. If the stream-wise fluctuations become negative, the distribution of the wall-normal velocity component decreases slowly while the shape of the graphs becomes non Gaussian and the spectrum around the maximum decreases. In connection with the sign of the the fluctuations, this again implies that low-speed structures are more frequently associated with a positive wall-normal velocity component whereas the probability of finding strong high-momentum structures is related to a motion toward the wall. This can be explained simply by assuming that turbulent structures coming from large wall distances at $y+\Delta y$ conserve their momentum while travelling wall-ward [91]. Furthermore, the different decay of the maximum with increasing magnitude of $u$ should be noted as this indicates that the flow structures associated with positive wall-normal motion seem to be more coherent than the other structures. Finally, the fact is important that the magnitude of the largest stream-wise and wall-normal fluctuations are approximately twice as large as the corresponding rms value measured at the same $y^{+}$-position because this point supports the arguments used to explain the two maxima in the lower right plot of figure 6.1. Whereas the graphs in the lower plots, which represent the statistical structure of the $v w$ fluctuations, are nearly Gaussian and symmetrical in this representation, e.g. $\operatorname{PDF}(-v, w)=\operatorname{PDF}(v, w)$, the graphs of the centre row possess more or less pronounced shoulders which indicate a complex interaction of the structures in this region, but the nature of the processes cannot be identified from the single-point statistics. To deduce the typical flow angles associated with the transport of low and high momentum fluid towards and away from the wall, the probability density function of the absolute angle between the 

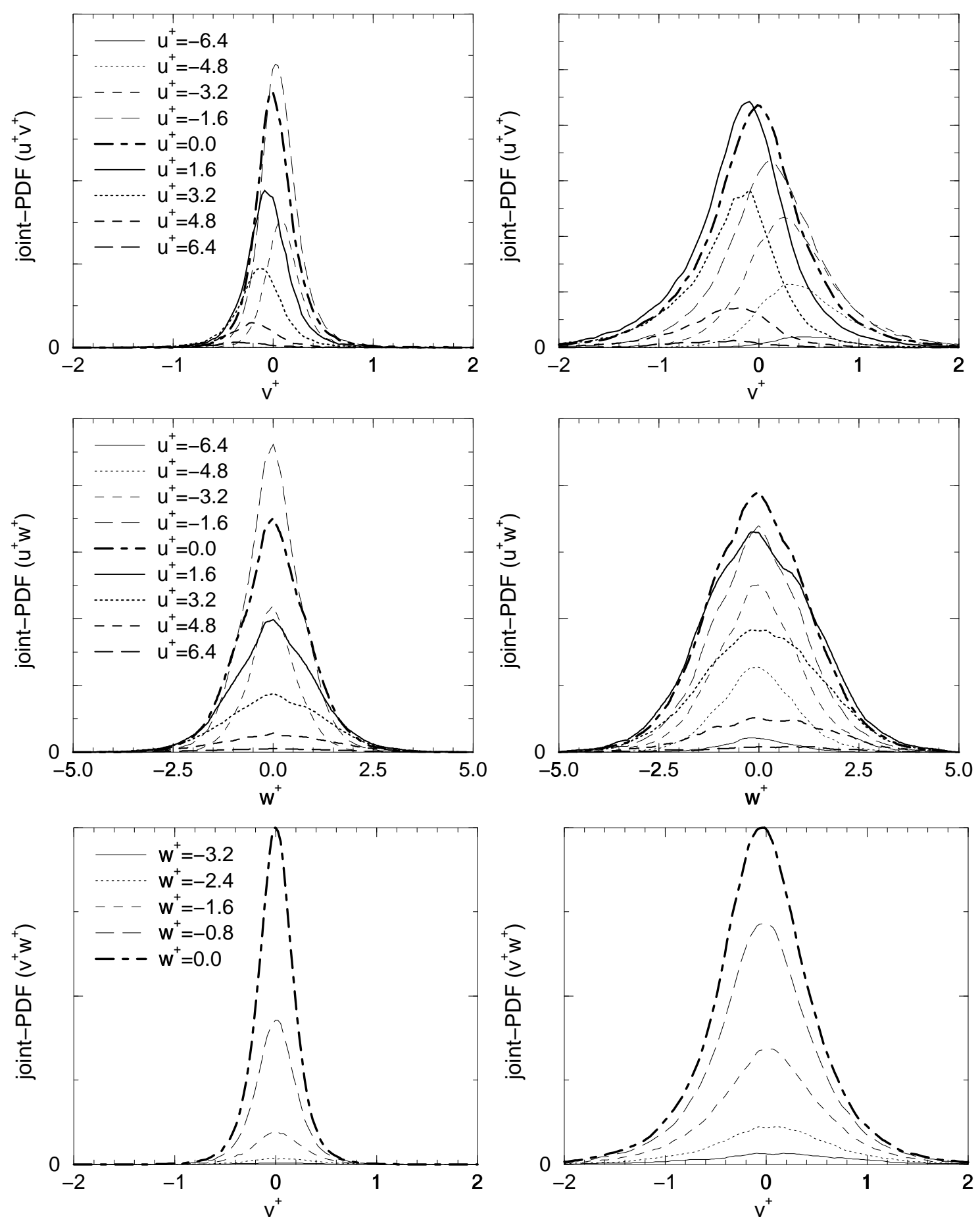

FIGURE 6.4: Joint-PDF functions of the $u^{+} v^{+}, u^{+} w^{+}$and $w^{+} v^{+}$(top to bottom) measured at $R e_{\theta}=$ 7800 and two wall-normal locations: $y^{+} \approx 10$ (left) and $y^{+} \approx 20$ (right).

instantaneous values of the various velocity components was calculated at $y^{+} \approx 10,20$ and 30 . The $w / U$-distribution, shown in the lower left graphs of figure 6.5 , is exactly symmetrical with respect to the centreline, and the range of angles increases when approaching the wall. This is clear as the magnitude of the span-wise velocity fluctuations increases slightly toward the wall according to figure 5.3. The $v / U$-distribution on the other hand is asymmetric with 

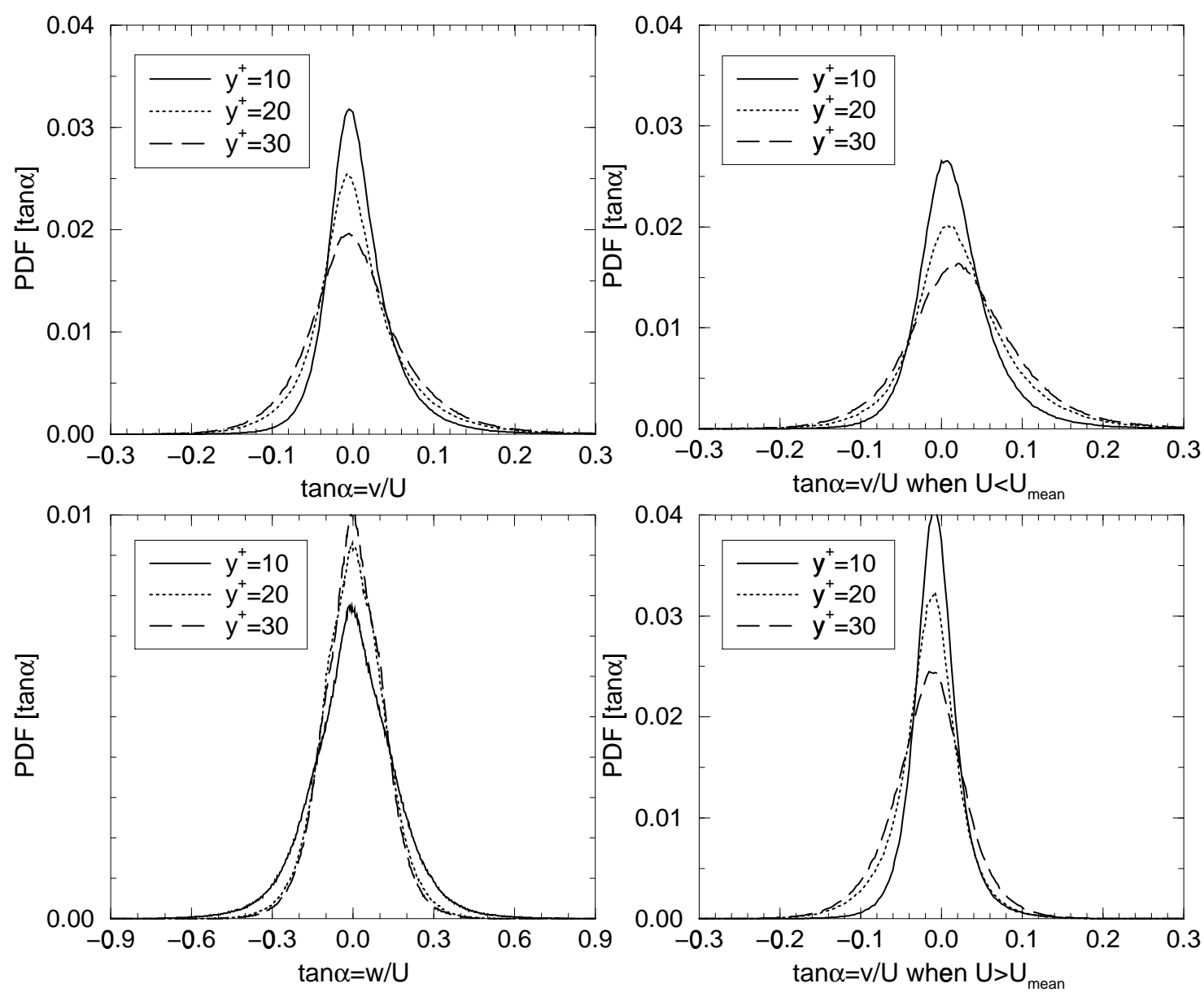

FIGURE 6.5: Probability density distribution of the flow angle between the instantaneous values of the various velocity components at $y^{+} \approx 10,20$ and $30 . v / U$ (upper left), $w / U$ (lower left), $v / U$ with $U<\bar{U}$ (upper right), $v / U$ with $U>\bar{U}$ (lower right).

a large range of scales for positive angles and a maximum located at negative $\alpha$ in agreement with [61]. The right column of figure 6.5 shows the distribution of the characteristic angles for the velocity structures whose instantaneous stream-wise velocity $U$ is below (upper graphs) or above the mean motion (lower graphs). As a stream-wise velocity which is larger than the mean velocity indicates a positive fluctuation $u$, the positive domain of the lower right plot indicates high-speed flow structures which move away from the wall, whereas the negative part indicates large momentum fluid moving towards the wall. The asymmetry of the measurements clearly indicates that the probability of finding a large momentum fluid moving towards the wall (so called sweeps according to section 5.4.3, or $Q 4$ events based on the definitions on page 71) is quite high relative to high-speed fluid moving away from the wall. This implies the dominance of $Q 4$ events over $Q 1$ when large magnitude events are considered, and the upper right plots state that $Q 2$ events or ejection dominate over $Q 3$ movements. Furthermore, it should be noted that the largest angles are always associated with $Q 2$ and $Q 4$ events.

Table 6.3 reveals the statistical properties of the absolute flow angles between the instantaneous velocity components with respect to the stream-wise direction for three different wall locations. The angles are quite small in relation to values from other experiments and numerical flow simulations [86] as here the instantaneous stream-wise flow velocity $U$ was used to 
calculate the absolute flow angles. The angles become comparable with the literature when the stream-wise velocity fluctuation $u$ is applied for the normalisation. This will be shown later. Due to the strong dynamic of the velocity fluctuations in the buffer layer it is not evident if the convection term in the conservation equations is large relative to the sum of the production, diffusion and dissipation term, as required for the observation of coherent structures. However, as the statistical results examined in this section indicate a strong relation between various velocity fluctuations, it seems likely that the results are related with the motion of organised or coherent flow structures. These structures, whose existence was already assumed by Prandtl in 1925 for the derivation of the mixing-length theory, can be defined as flow regions over which the simultaneous velocity fluctuations are correlated.

\begin{tabular}{lccc}
\hline & $y^{+}$ & $\alpha_{\text {mean }}[\mathrm{deg}]$ & $\alpha_{\mathrm{rms}}[\mathrm{deg}]$ \\
\hline \multirow{3}{*}{$v / U$} & 10 & 0.38 & 2.57 \\
& 20 & 0.23 & 3.36 \\
& 30 & 0.30 & 3.75 \\
\hline \multirow{3}{*}{$v /(U<\bar{U})$} & 10 & 1.00 & 2.86 \\
& 20 & 1.43 & 3.76 \\
& 30 & 1.59 & 4.20 \\
\hline \multirow{3}{*}{$v /(U>\bar{U})$} & 10 & -0.36 & 1.90 \\
& 20 & -0.90 & 2.43 \\
& 30 & -0.87 & 2.79 \\
$w / U$ & 10 & 0.17 & 9.32 \\
& 20 & 0.14 & 6.84 \\
& 30 & 0.13 & 6.42 \\
\hline
\end{tabular}

TABLE 6.3: Statistical properties of the absolute flow angles between the instantaneous velocity components with respect to the stream-wise direction measured at $y^{+}=10,20,30$.

\subsubsection{Spatial auto-correlation functions}

In order to link the statistical results of the previous section with the concept of coherent flow structures, outlined in chapter 1 and section 5.4, the properties of normalised spatial correlation and cross-correlation functions will be considered in this section, for theoretical details see [53]. First of all, the average dimensions of the dominant flow structures will be deduced from the primary components of the double correlation tensor measured in stream-wise spanwise planes, at $y^{+}=10,20,30$. The results can be seen as a completion of the stereoscopic PIV investigation described in $[38,44]$, where the planes at $y^{+}=30,100,220$ were investigated at $R e_{\theta}=950$ and 7800 . Unfortunately, a quantitative comparison with the large-scale hot-wire investigation by Grant [24] is questionable because of the different flow conditions and wall locations as already mentioned on page 79 . However, his arguments for the interpretation of the primary correlations will be applied and its validity for the near-wall correlations will be proven. The contours of the following plots are spaced in intervals of 0.05 for the primary correlation (excluding 0.05 and 1) and the position and amplitude of the minimum and maximum are included for comparison. Continuous lines indicate positive correlation whereas dotted lines assign regions with negative correlation values. Figure 6.6 shows the primary auto-correlations of the stream-wise (left column) and wall-normal (right column) 


\section{Investigation of the $x z-p l a n e$}

velocity fluctuations measured at $y^{+} \approx 30,20$ and 10 (top to bottom). Clearly noticeable is the characteristic elliptical shape of both correlations, with the principal axis in stream-wise direction, as well as the strong dependence of the correlation width from the wall distances in accordance with [44] and the results presented in section 5.3.2. However, the most striking feature, which could not be established before, is the variation of the size and span-wise location of the weak negative correlation regions of $R_{v v}$, when approaching the wall. This is clearly visible in the upper right plot of figure 6.7 where the span-wise dependence of this correlation at $\Delta x^{+}=0$ is shown in wall-units. The location of the minima is highlighted with symbols and the legend reveals the corresponding displacement of the symbols from the maximum of correlation, located at $\Delta z^{+}=0$. The range of correlation values indicates a large range of scales and the minima imply a span-wise periodicity whose wavelength strongly increases with increasing wall distance. From the negative correlation regions, the existence of stream-wise vortices can be concluded. However, it should be noted that their length is not
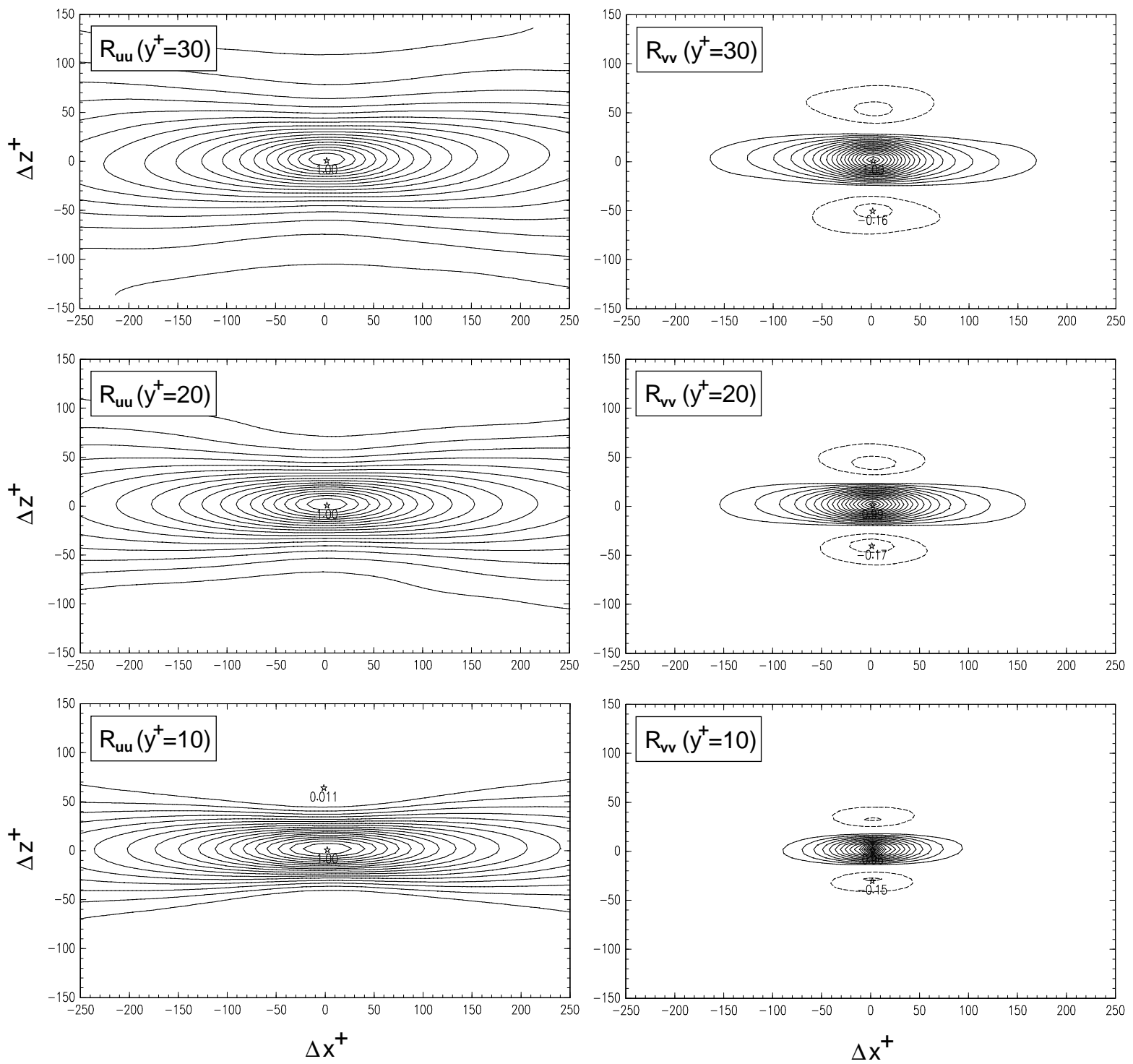

FIGURE 6.6: Two-dimensional spatial correlation function of fluctuating stream-wise velocity $R_{u u}$ (left) and wall-normal velocity $R_{v v}$ (right) measured at $y^{+} \approx 30,20$ and 10 (top to bottom). 
as long as expected by other authors as can be seen from the stream-wise extension of the negative correlation region in figure 6.6. In case of the $R_{u u}$ correlation, shown in the upper left plot of figure 6.7 , only the function measured at $y^{+} \approx 10$ changes its sign in span-wise direction and reveals a weak minimum at $\Delta x^{+} \approx 63$, while the others slowly converge to zero with increasing $\Delta z^{+}$. This indicates a decreasing dynamic velocity range with increasing wall distance, as expected from the $\sqrt{\overline{u^{2}}}$ profiles in figure 5.3, and implies that the coherent velocity regions rapidly loose their identity with increasing wall distance $y$. To deduce the statistical properties of the dominant low- and high-momentum flow structures which are convecting downstream, the measured data was subdivided according to the sign of the stream-wise velocity fluctuation before calculating the correlation functions. This conditional correlation approach allows to identify the structures associated with ejection and sweeps for instance.
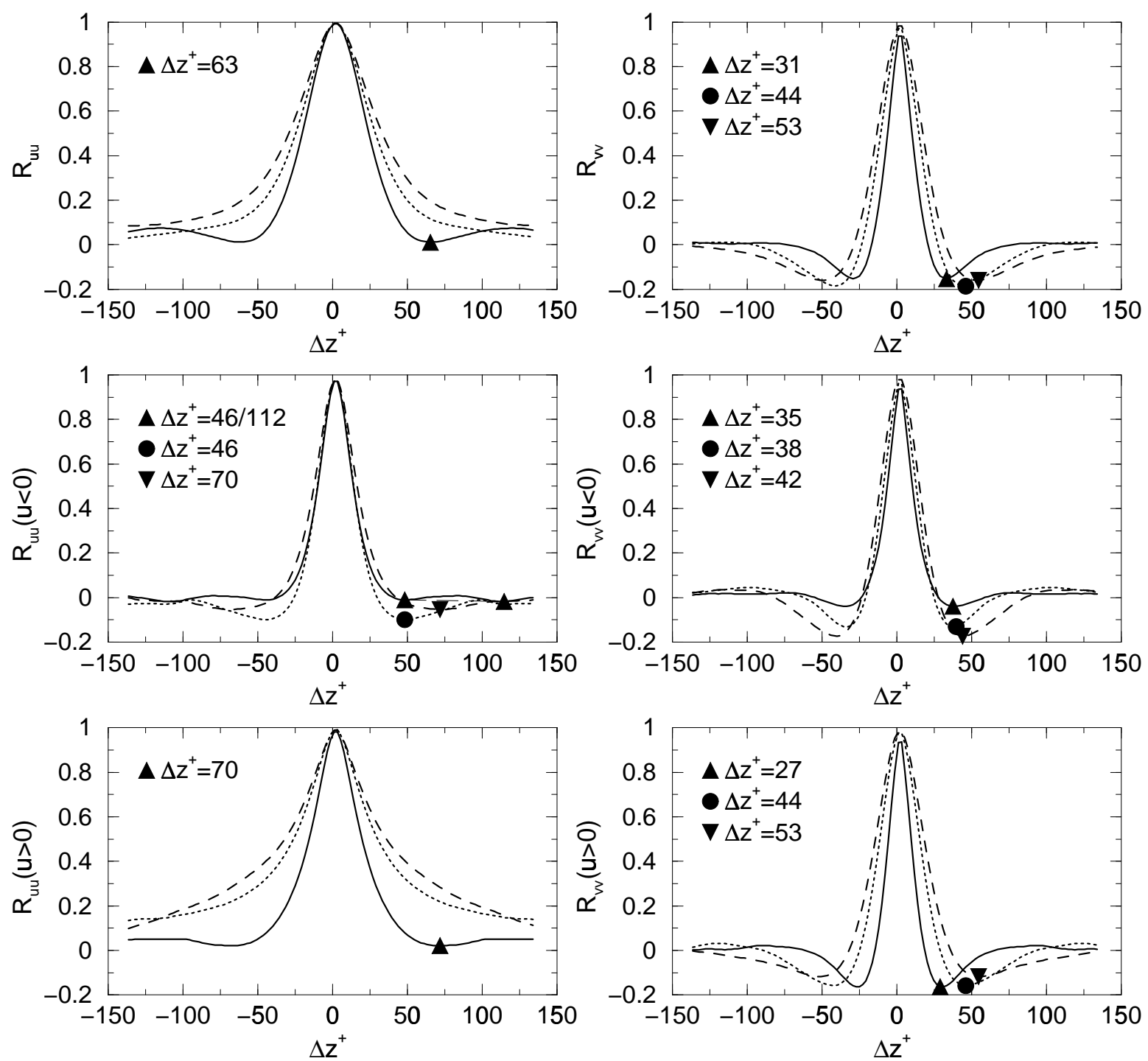

FIGURE 6.7: One-dimensional spatial correlation function of fluctuating stream-wise and wall-normal velocity components measured at $y^{+} \approx 10$ (solid graph), $y^{+} \approx 20$ (dotted graph) and $y^{+} \approx 30$ (dashed graph) as a function of the span-wise coordinate. The symbols indicate the location of the minimum and the legend the displacement of the minimum from the origin. 
The left plot of the centre row of figure 6.7 reveals the $R_{(u<0)(u<0)}$ correlations, denoted by $R_{(-u)(-u)}$ for simplicity, and the right plot shows the associated correlation of the positive and negative wall-normal fluctuations in the particular regions where $u$ is negative. The coherent velocity regions selected in this way show nearly the same functional dependence around the maximum (down to correlation values of 0.2 for $R_{(-u)(-u)}$ and nearly zero for $R_{v v}(u<0)$ ), which indicates a high degree of coherence in wall-normal direction. When the $R_{v v}(u<0)$ correlation is considered the value of the minimum increases with increasing wall distance. This is completely different when only the correlations of the wall-normal fluctuations are calculated which can be measured in regions where the stream-wise velocity fluctuations are larger than the mean velocity, e.g. $R_{v v}(u>0)$ as shown in the lower right plot of the same figure. In this case the value of the minimum decreases with increasing wall distance. The symbol explanation of the $R_{v v}(u<0)$ and $R_{v v}(u>0)$ correlations in the figures indicates that the distance between the minima and the maximum increases with increasing wall distance for both functions but faster for the $R_{v v}(u>0)$, especially close to the wall. Moreover, it can be concluded from the functional dependence of the correlations around the maximum that the coherence of the $R_{v v}(u>0)$ structures is rather weak compared to the coherent structures represented by $R_{v v}(u<0)$. Also, the structures which enter in the calculation of $R_{(-u)(-u)}$ loose their identity to a large extent with increasing wall-distance as indicated by the strong difference between the graphs. The exact value of the span-wise periodicity of the structures, indicated by the minimum in the correlations, is still a point of discussion in the literature, especially at high Reynolds numbers. One reason for this controversy is the dependence of this number on the method applied for the determination of the wavelength. However, it should be noted that the conditional correlation approach, applied here, is fully based on mathematical grounds and yields only one well defined value for the wavelength, instead of a streak-spacing distribution from which a mean has to be calculated in an appropriate way. Table 6.4 reveals the values measured in air- and water-facilities at different Reynolds number by using various detection methods. It can be seen that the exact spacing extracted from the $R_{v v}, R_{v v}(u<0)$ and $R_{v v}(u>0)$ correlation in figure 6.7, does not match with the values found in the cited work but when the $R_{(-u)(-u)}$ correlation is considered at $y^{+} \approx 10$, the agreement is remarkable with the other experiments.

TABLE 6.4: Comparison of streakspacing and flow parameters. First block: air, PIV, conditional correlation method, $y^{+} \approx 10$, [Present study]. Second block: water, hydrogen bubbles, $y^{+} \approx 5$, see [93]. Third block: air, probe rake, $y^{+} \approx 2-7$, see [25]. Forth block: water, hydrogen bubbles, $y^{+} \approx 4$, see [92].

\begin{tabular}{ccccr}
\hline$R e_{\theta}$ & $U_{\infty}[\mathrm{m} / \mathrm{s}]$ & $u_{\tau}[\mathrm{m} / \mathrm{s}]$ & $x[\mathrm{~m}]$ & $\lambda_{z}^{+}$ \\
\hline 7800 & 3.0 & 0.1210 & 18.0 & 92 \\
\hline 3160 & 0.390 & 0.0155 & 4.50 & 96 \\
3310 & 0.305 & 0.0121 & 4.27 & 104 \\
4180 & 0.387 & 0.0150 & 4.27 & 93 \\
4940 & 0.475 & 0.0181 & 4.27 & 97 \\
5830 & 0.582 & 0.0220 & 4.27 & 95 \\
\hline 3300 & - & 0.2256 & - & 89 \\
4700 & - & 0.3277 & - & 110 \\
\hline 1080 & 0.152 & 0.0070 & 3.14 & 91 \\
1325 & 0.152 & 0.0069 & 4.11 & 106 \\
\hline
\end{tabular}




\subsubsection{Spatial cross-correlation functions}

For phenomena associated with the production of turbulence the cross correlation $R_{v u}$ is more important than the primary components of the correlation tensor, as $R_{v u}$ reflects the size and shape of the structures being responsible for the transport of relatively low-momentum fluid outwards into higher speed regions and for the movement of high-momentum fluid toward the wall and into lower speed regions. This correlation was first studied by Tritton in 1967 by using a pair of hot-wire probes $2.1 \mathrm{~m}$ behind a trip-wire at free-stream velocities between $6.75 \mathrm{~m} / \mathrm{s}$ and $7.1 \mathrm{~m} / \mathrm{s}$ [100]. The boundary layer thickness was $60 \mathrm{~mm}$ and the Reynolds number $R e_{\delta}=22000$. Due to technical reasons his results were only measured along the three principal axes and at larger wall-locations, so that a direct comparison with the work presented here is questionable. In the following, it is important to keep in mind that the cross-correlation
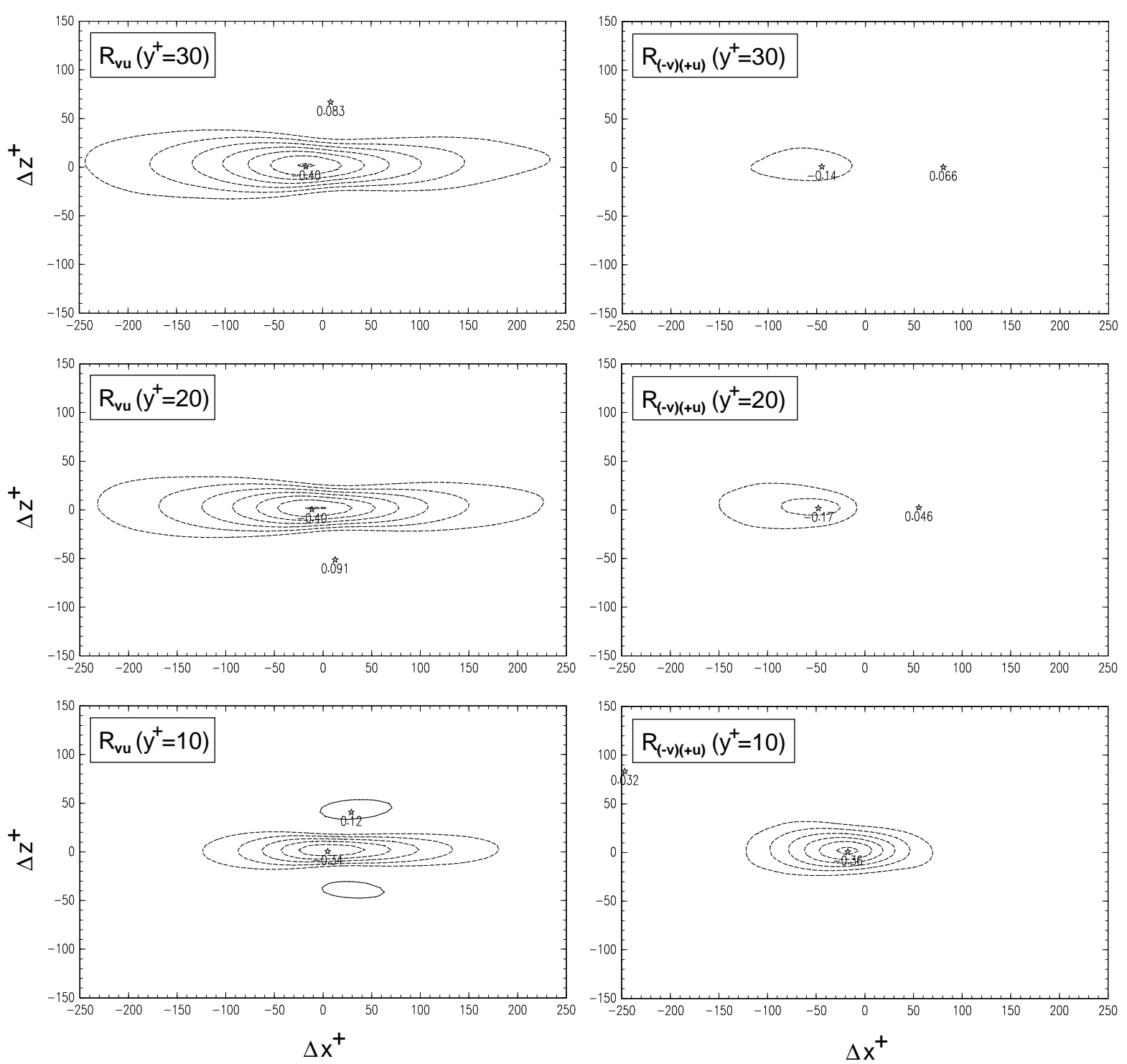

FIGURE 6.8: Two-dimensional spatial cross-correlation function of fluctuating stream-wise with wallnormal velocity components $R_{v u}$ (left) and conditional cross-correlation of negative wall-normal with positive stream-wise velocity $R_{(-v)(+u)}$ (right) measured at $y^{+} \approx 30,20$ and 10 (top to bottom). 
function is not necessarily an even function with a maximum at $\Delta x_{i}=0$ in contrast to the primary correlations, but there exists still an important symmetry property when the random variables $u$ and $v$ are interchanged, namely $R_{u v}\left(\Delta x_{i}\right)=R_{v u}\left(-\Delta x_{i}\right)$. The left column in figure 6.8 shows the $R_{v u}$ correlation function with the $v$ component fixed and $u$ shifted in the two homogeneous directions. First of all it should be noted that the sign of $R_{v u}$ again indicates that the transport of relatively low-momentum fluid outward into higher speed regions $(u<0$ and $v>0$ ) and the movement of high-momentum fluid toward the wall into lower speed regions $(u>0$ and $v<0)$ are the predominant processes in the near-wall region. In addition, the strong elliptical shape implies that the turbulent mixing in the wall-normal direction is related to the low-speed structures represented by $R_{(-u)(-u)}$ in figure 6.7. But obviously only a small part of the low-momentum structures shows a correlated motion in both
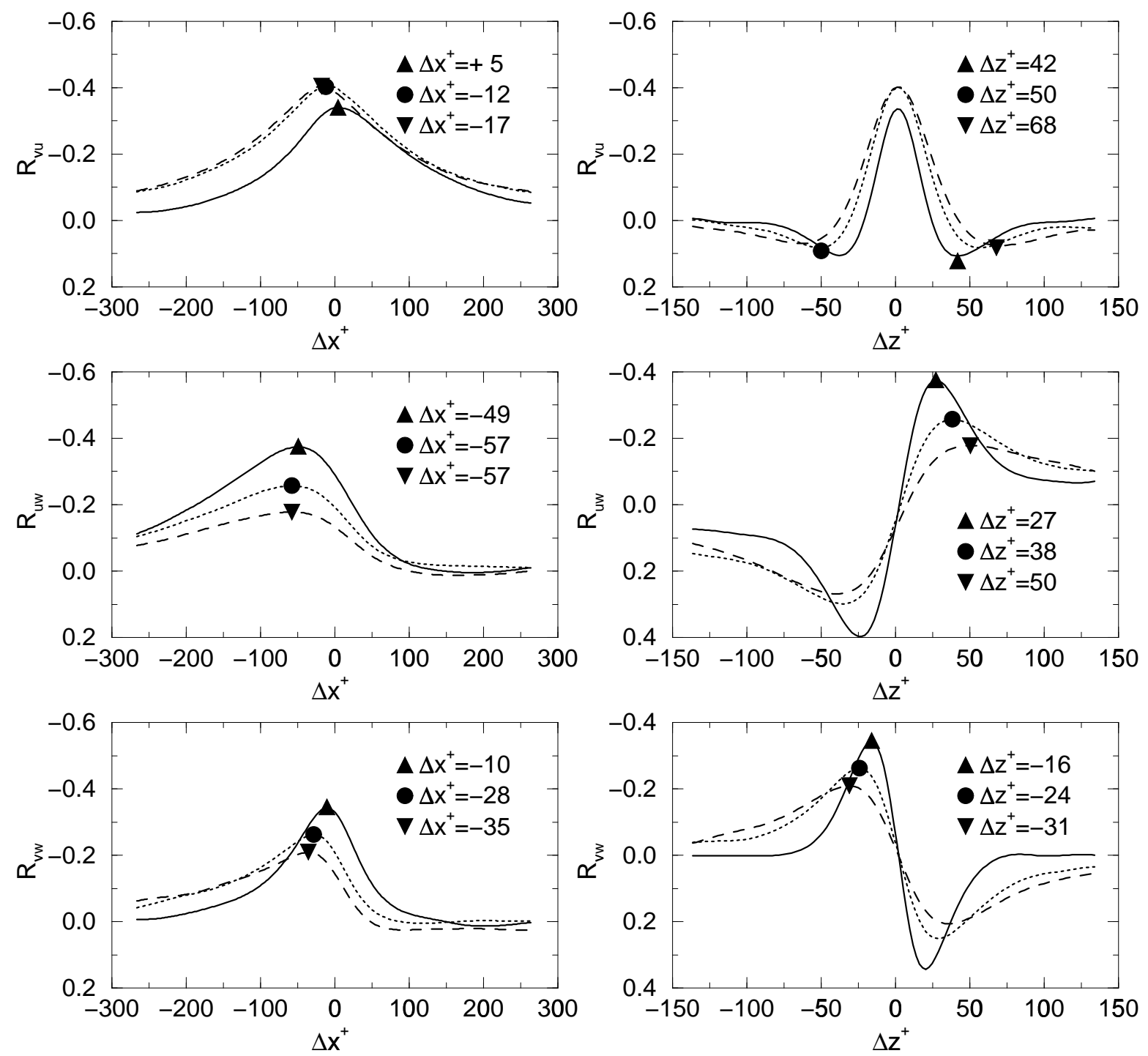

FIGURE 6.9: One-dimensional spatial cross-correlation function of Reynolds stress components measured at different wall-normal location (Solid graph: $y^{+} \approx 10$. Dotted graph: $y^{+} \approx 20$. Dashed graph: $\left.y^{+} \approx 30\right)$ as a function of $\Delta x^{+}$and $\Delta z^{+}$. The symbols indicate the maximum of correlation and the legend the distance from the minimum to the origin. 
stream-wise and wall-normal direction with sufficient strength (lower curve of figure 6.8) as the total length of the low-speed structures are several thousand wall-units in length according to the size of the $R_{u u}$ correlation, shown in figure 6.5. This is fully consistent with the size of the $R_{v v}$ correlation and the results in [44]. The span-wise extent of the flow-structures associated with the production of turbulence can be estimated best from the upper right graphs of figure 6.9 and the phase relation between both orthogonal fluctuations from the location of the maximum shown in the upper left graphs of the same figure. Apart from the structural details of the flow regions associated with the turbulent exchange in wall-normal and stream-wise direction, it is important to consider the cross-correlation with the span-wise velocity fluctuations because any outflow of fluid induces an organised span-wise flow motion towards the lifting structure due to continuity. In addition, as in isotropic turbulence this quantity must be zero, these correlations indicate any departure from this ideal situation which is mathemati-
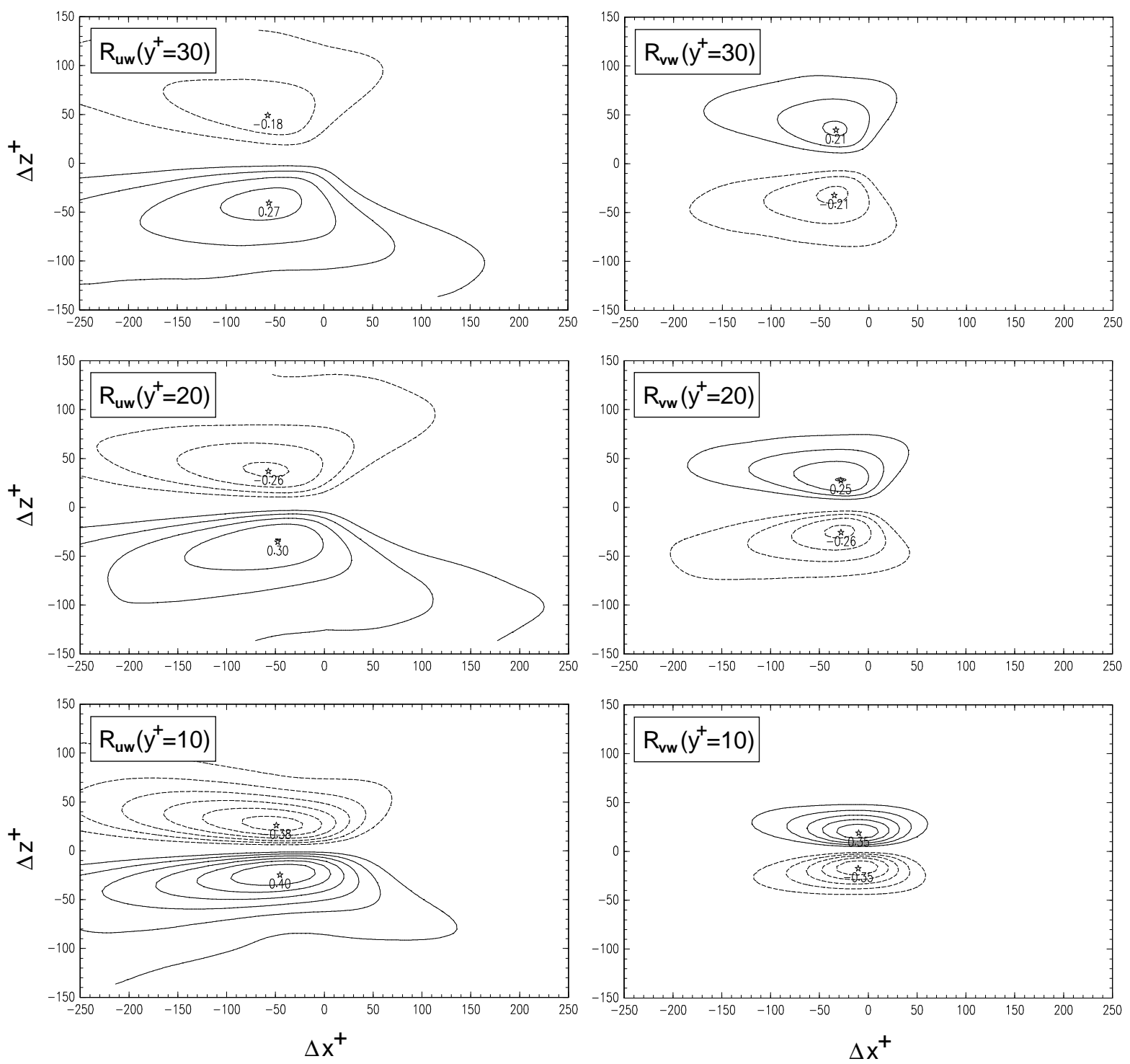

FIGURE 6.10: Two-dimensional spatial cross-correlation function of fluctuating stream-wise with span-wise velocity components $R_{u w}$ (left) and correlation of wall-normal with span-wise components $R_{v w}$ (right) measured at $y^{+} \approx 30,20$ and 10 (top to bottom). 


\section{Investigation of the $x z-p l a n e$}

cally accessible. Figure 6.10 shows the two-dimensional spatial cross-correlation function of fluctuating stream-wise with span-wise velocity components $R_{u w}\left(\Delta x^{+}, y^{+}, \Delta^{+} z\right)$ (left) and correlation of wall-normal with span-wise components $R_{v w}\left(\Delta x^{+}, y^{+}, \Delta z^{+}\right)$(right) measured at $R e_{\theta} \approx 7800$ and $y^{+} \approx 30,20,10$ (top to bottom). First of all, it can be concluded from the general size, shape and height of both correlations, that a very well organised span-wise motion exists, especially at $y^{+} \approx 10$ where the height of $R_{v w}$ is above the $R_{v u}$ correlation. This organised motion implies that the concept of local isotropy, which links the turbulent-energy dissipation in shear flows with the dissipation of isotropic turbulence, is inadequate in nearwall turbulence. Secondly, it should be noted that the degree of organisation increases with decreasing wall distance while the opposite holds for the $R_{v u}$ correlation as can be estimated from the intensity of the maximum. Thirdly, the spacing between the extrema is increasing with larger wall distances but also, it can be seen that the location of these particular points
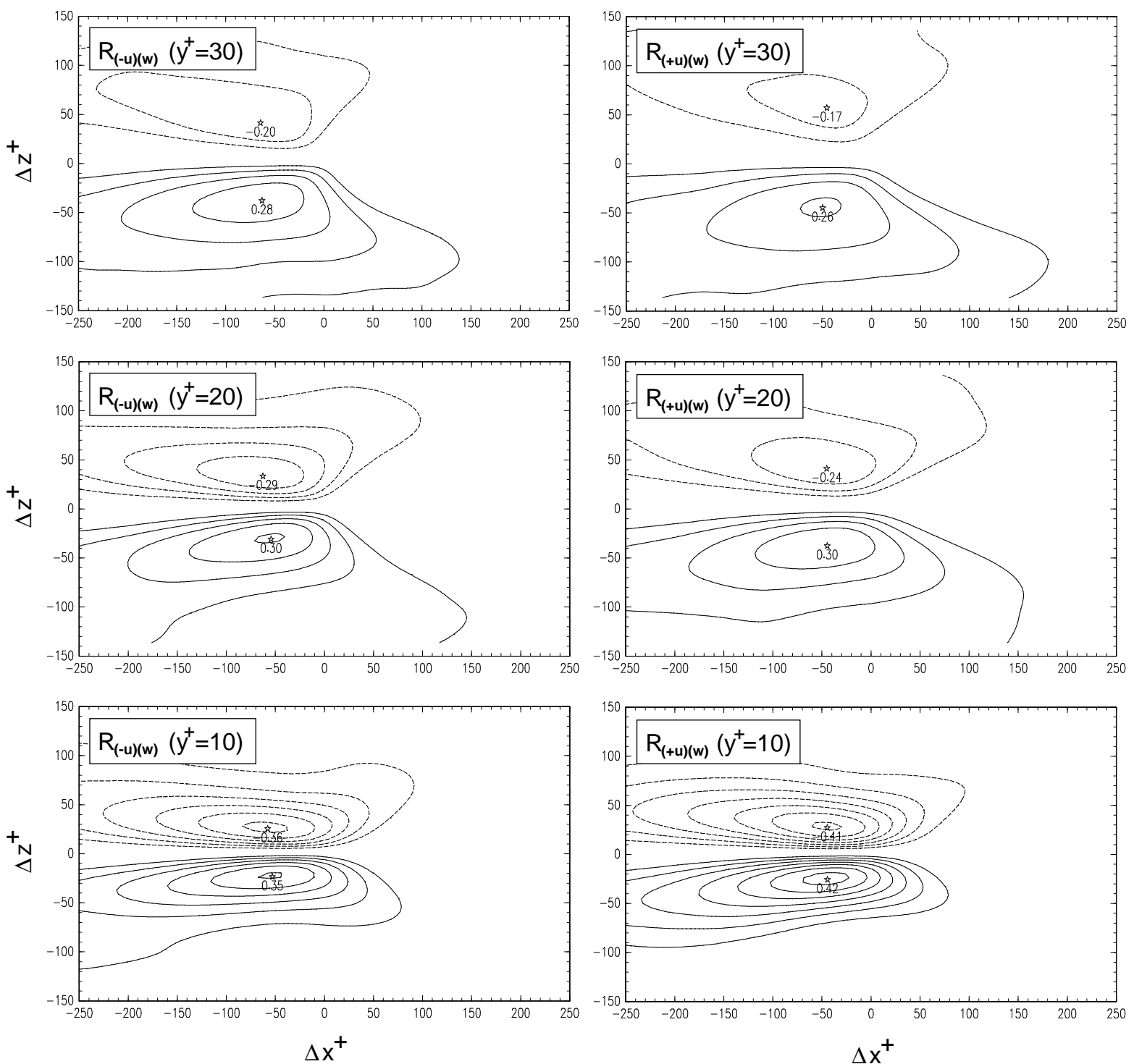

FIGURE 6.11: Conditional cross-correlation of negative stream-wise fluctuations with the span-wise velocity component $R_{(u<0)(w)}$ (left) and correlation positive stream-wise fluctuations with span-wise components $R_{(u>0)(w)}$ (right) measured at $R e_{\theta}=7800$ and $y^{+}=30,20,10$ (top to bottom). 
moves to negative stream-wise displacements, particularly if the $R_{v w}$ correlation is considered. For clarity, the two lower rows of figure 6.9 reveal the functional dependence of the correlations along the axis of symmetry. This representation allows to compare the height of the correlation and the location of the maximum in detail. Figure 6.11 shows the twodimensional spatial cross-correlation function of negative stream-wise fluctuations with the span-wise velocity component $R_{-u w}\left(\Delta x^{+}, y^{+}, \Delta z^{+}\right)$(left) and correlation positive streamwise fluctuations with span-wise components $R_{+u w}\left(\Delta x^{+}, y^{+}, \Delta z^{+}\right)$(right). Figure 6.12 displays the corresponding function of positive wall-normal fluctuations (extracted from flow regions where $u<0$ ) with the span-wise velocity component $R_{+v w}\left(\Delta x^{+}, y^{+}, \Delta z^{+}\right)$(left) and correlation of negative wall-normal fluctuations (extracted from flow regions where $u>0$ ) with span-wise components $R_{v w}$ with $(v<0)$ (right). These structures permit to resolve the
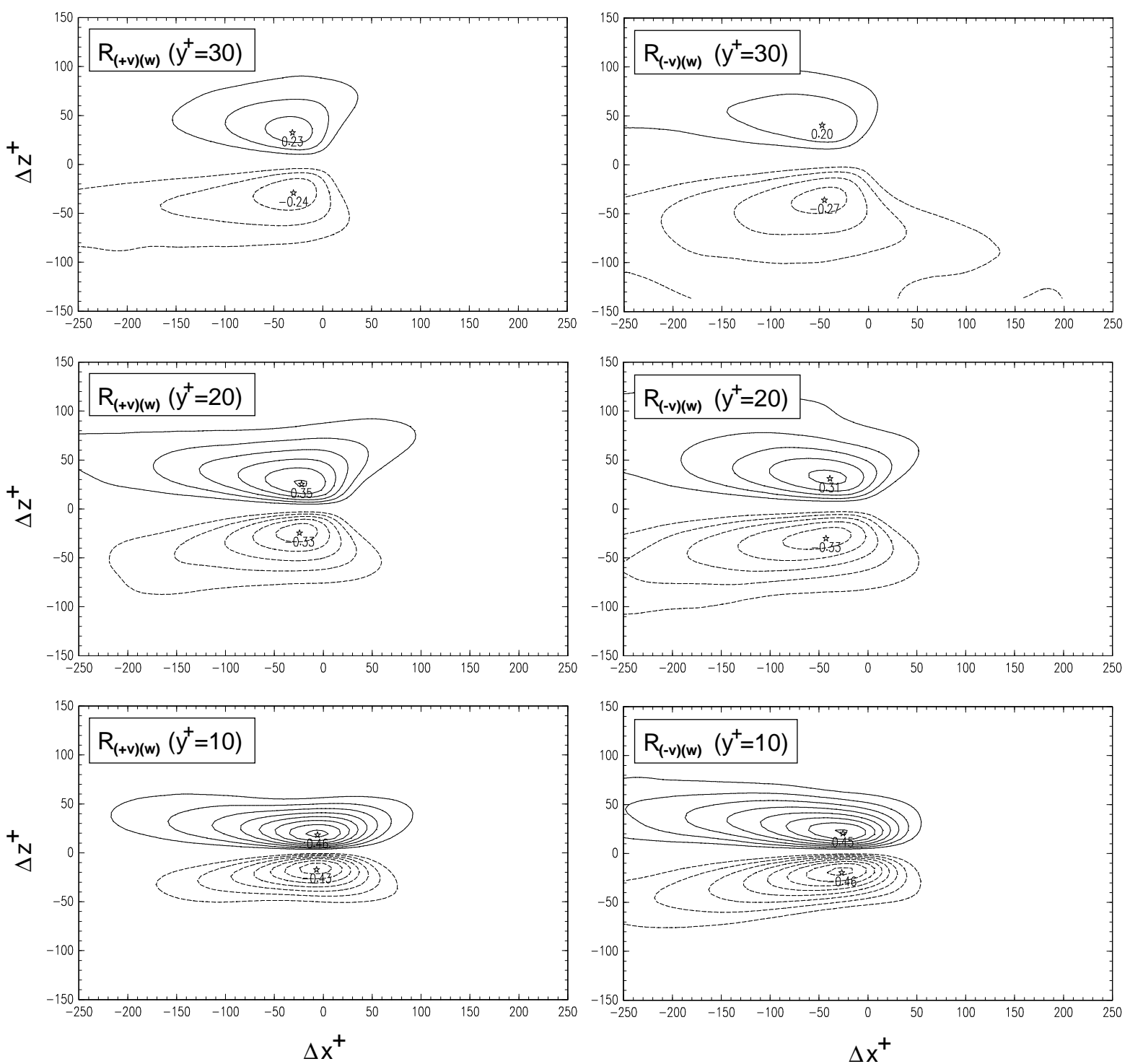

FIGURE 6.12: Conditional cross-correlation of positive wall-normal fluctuations (extracted from flow regions where $u<0$ ) with the span-wise velocity component $R_{(v>0)(w)}$ (left) and correlation of negative wall-normal fluctuations (extracted from flow regions where $u>0$ ) with span-wise components $R_{(v<0)(w)}$ (right) measured at $R e_{\theta}=7800$ and $y^{+}=30,20,10$ (top to bottom). 
processes associated with the organised motion in span-wise direction in more detail. Especially the value of maximum correlation should be noted for different wall distances and with respect to $R_{u w}$ and $R_{v w}$. In order to interprete the various correlation patterns qualitatively, it is necessary to assume a pure flow motion indicated by the first subscript (motion towards the wall in case of $R_{(v<0)(w)}$ ) at the origin of the correlation plane. Using this method it becomes evident that a motion towards the wall is on average associated with a span-wise motion away from the structure while the opposite holds for an organised motion away from the wall (compare the sign of the structures). The location of the maxima, on the other hand, implies that a phase relation exists between the two motions. When the flow direction is taken into account, it becomes apparent that the span-wise motion can be considered as the footprint of the wall-ward motion. Thus, the span-wise motion can be considered as a secondary motion. It is clear that the processes described are associated with two stream-wise vortices whose length is determined by the flow region moving in $y$-direction. For clarity the main features of the non-conditional functions have been summarised in figure 6.9 in form of one-dimensional graphs extracted at the location of the maximum of correlation in $x$ - and $z$-direction. This representation allows to compare quantitatively the height of the correlation and the location of the maximum in wall-units.

\subsection{Spatio-temporal buffer layer statistics}

In this section the spatio-temporal behaviour of the flow structures present in the buffer layer will be analysed quantitatively to examine their convection velocity and temporal decay. The first investigation of this type was performed by Favre et al (1957) who studied the spatiotemporal structure of the stream-wise velocity component $R_{u u}$ at $y^{+}>40$ and $R e_{\theta}=1600$, by using a pair of spatially separated hot wire probes $[19,20]$. Their measurements were performed $0.79 \mathrm{~m}$ and $1.94 \mathrm{~m}$ behind a tripping device at free-stream velocities of $12 \mathrm{~m} / \mathrm{s}$ with $\delta \approx 17 \mathrm{~mm}$ and $\delta \approx 34 \mathrm{~mm}$. Here, the structures at $y^{+}=30$ and below will be investigated at $R_{\theta}=7800$ by analysing the primary correlations $\left(R_{u u}, R_{v v}\right.$ and $\left.R_{w w}\right)$, the different crosscorrelations $\left(R_{u v}, R_{u w}\right.$ and $\left.R_{v w}\right)$ and conditional correlations taking into account the sign

of the fluctuations $\left(R_{+u v}, R_{-u v}, R_{+v w}, R_{-v w}\right.$ and others). The conditional correlations yield information about the space-time structure of the bursting phenomenon and allow to estimate the mean convection velocity of the coherent velocity structures in the near-wall region. This becomes important for proving the validity of Taylor's hypothesis in the near-wall region of a turbulent boundary layer flow. In other words, when the characteristic velocity of the large scale structure motions differs from the velocity of the small scale structures, the space-time transformation $\partial u / \partial t=-\bar{U} \partial u / \partial x$, usually applied when spatial derivatives are measured by using a hot-wire, becomes questionable.

All correlations considered so far were calculated at a fixed $y^{+}$location. Here, in contrast, the spatio-temporal correlation between separated planes is considered. The spacing is always 10 wall-units but the position of the lower measurement plane is altered between $y^{+} \approx 10$ and 20 wall-units. When the temporal delay between a pair of measurements is varied, this approach allows to investigate the structures moving toward and away from the wall, depending on the temporal order of the measurements. In other words, when a structure is moving towards the wall, this structure can be investigated best when the first measurement is performed at $y+\Delta y$ with $\Delta y>0$ and the second at $y$ while flow-structures moving away from the wall yield higher correlation values when the first measurement is 
performed at $y$ and the second at $y+\Delta y$. The basic requirement for the interpretation of the two-dimensional spatial cross-correlation functions is a clear understanding of the symmetry properties as their intensity, shape and position may strongly depend on the order of the fluctuating quantities considered for the correlation $R_{u v} \neq R_{v u}$ as well as on their exact position $R_{u(y+\Delta y) v(y)} \neq R_{u(y) v(y+\Delta y)}$. This is highlighted in figure 6.13 which reveals all possible combinations of the two-dimensional spatial cross-correlation functions of fluctuating stream-wise and wall-normal velocity components extracted simultaneously at $y^{+}=10$ and $y^{+}+\Delta y=20$. The important parameters for the analysis of the cross-correlation functions are their size, shape, intensity, sign and location of the maxima as these values furnish basically information about the average dimensions of the moving flow structures, about their degree of organisation and about their propagation direction relative to the mean motion. The particular example shown in figure 6.13 indicates that the correlation function is rather weak and relatively small in spatial extent when the $v$ component, measured in the plane located at $y^{+}=10$, is correlated with the $u$ component from the plane located at $y^{+}=20$ (see upper figure). The correlation of the $v\left(y^{+}=20\right)$ with $u\left(y^{+}=10\right)$, on the other hand, results in a quite strong and well extended correlation (see lower figure). In the lower left figure, the $u\left(y^{+}=10\right)$ component is shifted for the calculation of the cross-correlation function and for the lower right calculation the $v\left(y^{+}=20\right)$ component was shifted relatively to $u\left(y^{+}=10\right)$. The first series of two-dimensional spatial cross-correlations shown in figure 6.14 displays the
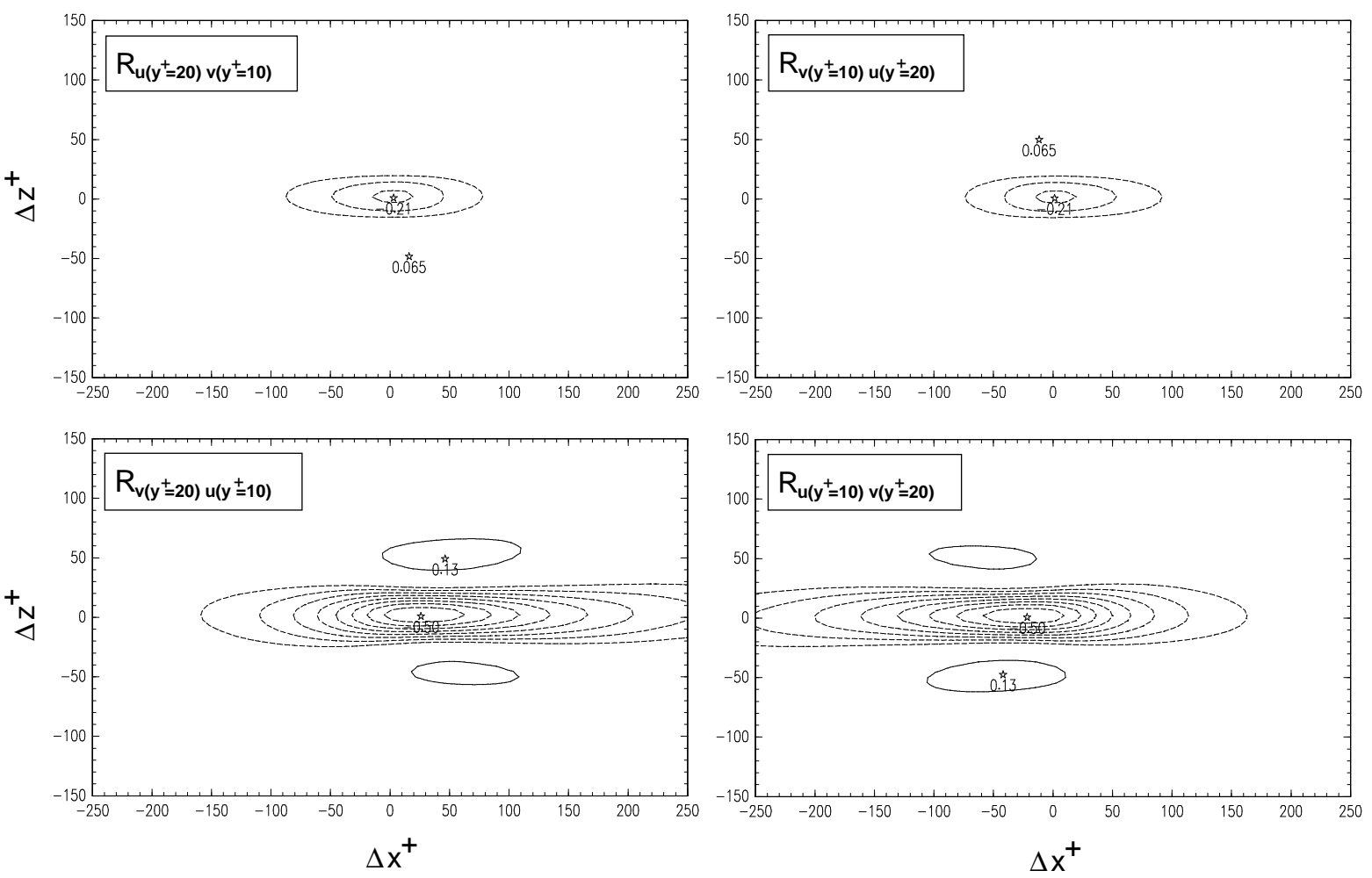

FIGURE 6.13: Possible two-dimensional spatial cross correlation functions of fluctuating stream-wise and wall-normal velocity components extracted simultaneously at $y$ and $y+\Delta y . R_{u(y+\Delta y) v(y)}$ (upper left), $R_{v(y+\Delta y) u(y)}$ (lower left), $R_{v(y) u(y+\Delta y)}$ (upper right), $R_{u(y) v(y+\Delta y)}$ (lower right).

statistical relation between the fluctuating stream-wise and the wall-normal velocity components, measured simultaneously at different $y^{+}$-locations. The top row displays the crosscorrelation $R_{v\left(y^{+}=20\right) u\left(y^{+}=10\right)}$ (left) and $R_{u\left(y^{+}=20\right) v\left(y^{+}=10\right)}$ (right). Again, it can be stated from 


\section{Investigation of the $x z-p l a n e$}

the negative sign of the correlation, indicated by the dashed lines, that the ejection and sweeps must be the predominant processes and the different size, shape and intensity of the functions $\left(R_{v\left(y^{+}=20\right) u\left(y^{+}=10\right)}>R_{u\left(y^{+}=20\right) v\left(y^{+}=10\right)}\right)$ imply the dominance of ejection at this wall locations. In addition it can be estimated from the location of the maximum in the upper left graph that the low momentum region appears as a shear layer in $y$-direction while the strong positive side peaks in the same figure indicate that the outflow of low-momentum fluid is associated with a secondary motion. The centre row of figure 6.14 reveals the same functions but measured at $y^{+}=20$ and $y^{+}=30$ e.g. $R_{v\left(y^{+}=30\right) u\left(y^{+}=20\right)}$ (left) and $R_{u\left(y^{+}=30\right) v\left(y^{+}=20\right)}$ (right). The bottom row of figure 6.14 yields the conditional cross-correlation $R_{v\left(y^{+}=20\right) u\left(y^{+}=10\right)}$ with $u>0$ (left) and $R_{v\left(y^{+}=30\right) u\left(y^{+}=20\right)}$ with $u>0$ (right). Especially the amplitude of $R_{v u}$ in the lower right plot should be noted.
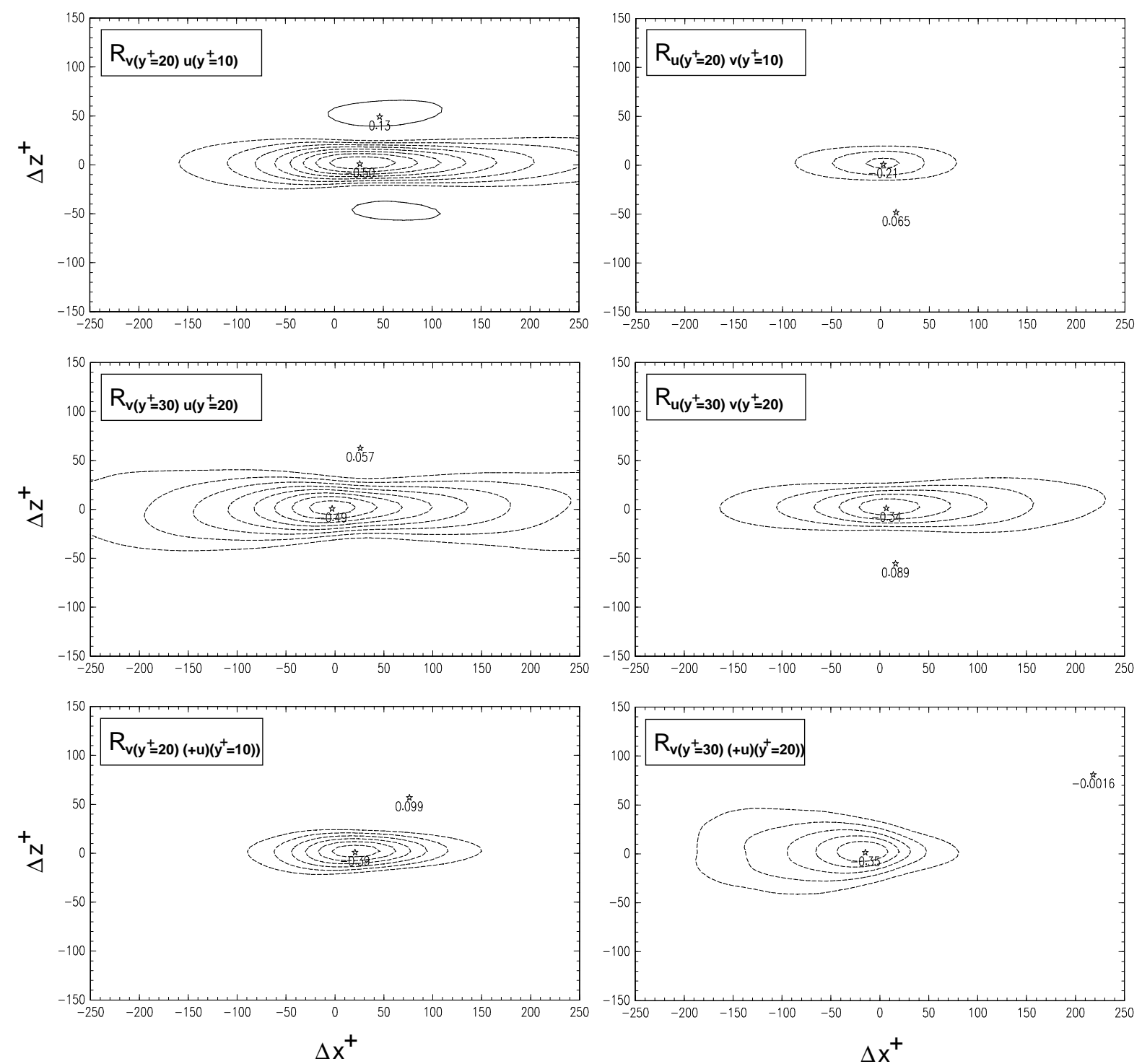

FIGURE 6.14: Two-dimensional spatial cross-correlation function of fluctuating stream-wise $(u)$ with wall-normal $(v)$ velocity components measured at $R e_{\theta}=7800$. Top: $R_{v\left(y^{+}=20\right) u\left(y^{+}=10\right)}$ (left) and $R_{u\left(y^{+}=20\right) v\left(y^{+}=10\right)}$ (right). Centre: $R_{v\left(y^{+}=30\right) u\left(y^{+}=20\right)}$ (left) and $R_{u\left(y^{+}=30\right) v\left(y^{+}=20\right)}$ (right). Bottom: $R_{v\left(y^{+}=20\right) u\left(y^{+}=10\right)}$ with $u>0$ (left $)$ and $R_{v\left(y^{+}=30\right) u\left(y^{+}=20\right)}$ with $u>0$ (right). 


\subsection{Spatio-temporal buffer layer statistics}
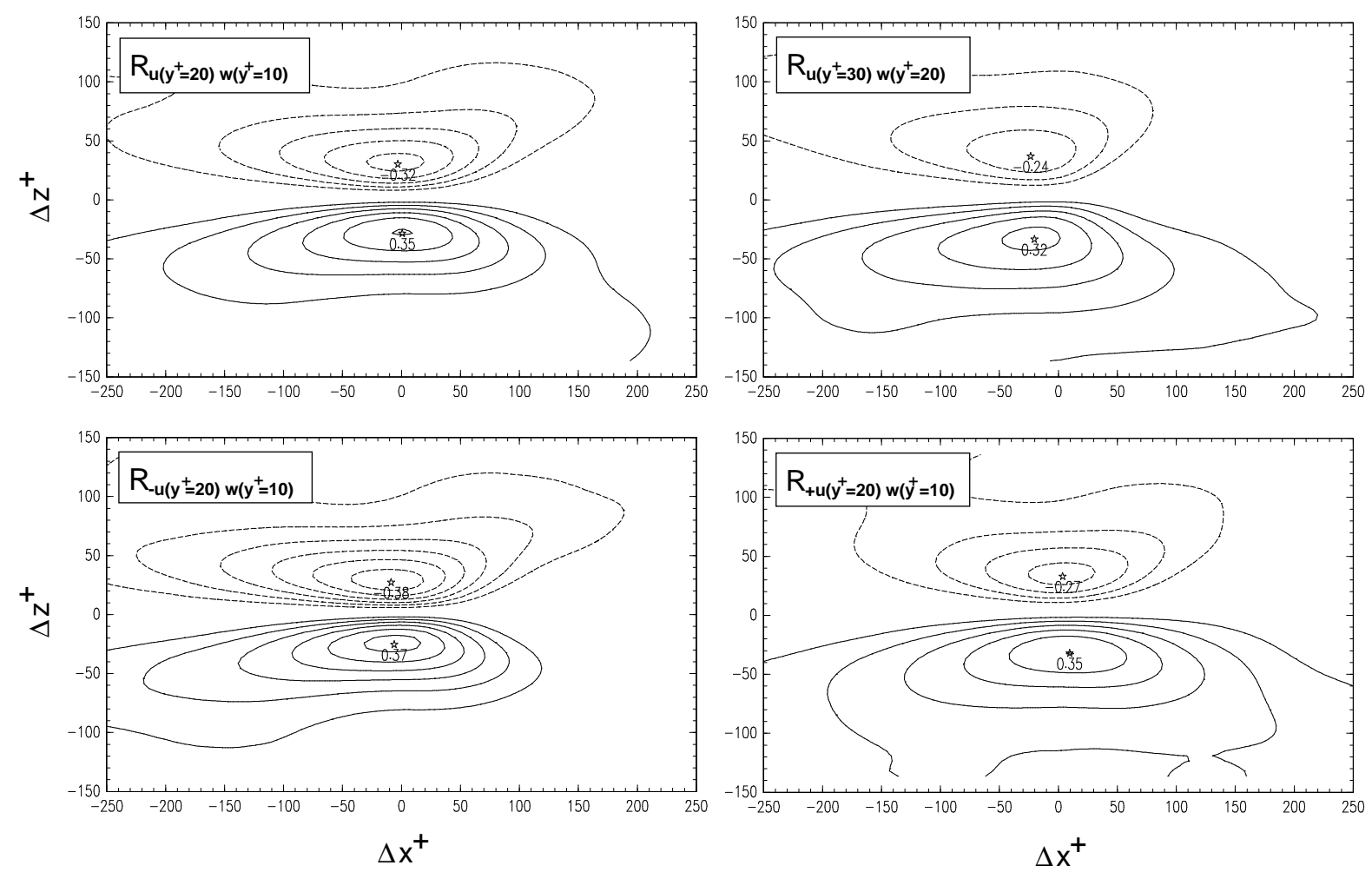

FigURE 6.15: Top: $\quad R_{u\left(y^{+}=20\right) w\left(y^{+}=10\right)} \quad$ (left) and $R_{u\left(y^{+}=30\right) w\left(y^{+}=20\right)}$ (right). Bottom: $R_{u\left(y^{+}=20\right) w\left(y^{+}=10\right)}$ with $u<0($ left $)$ and $u>0($ right $)$ measured at $R e_{\theta}=7800$.
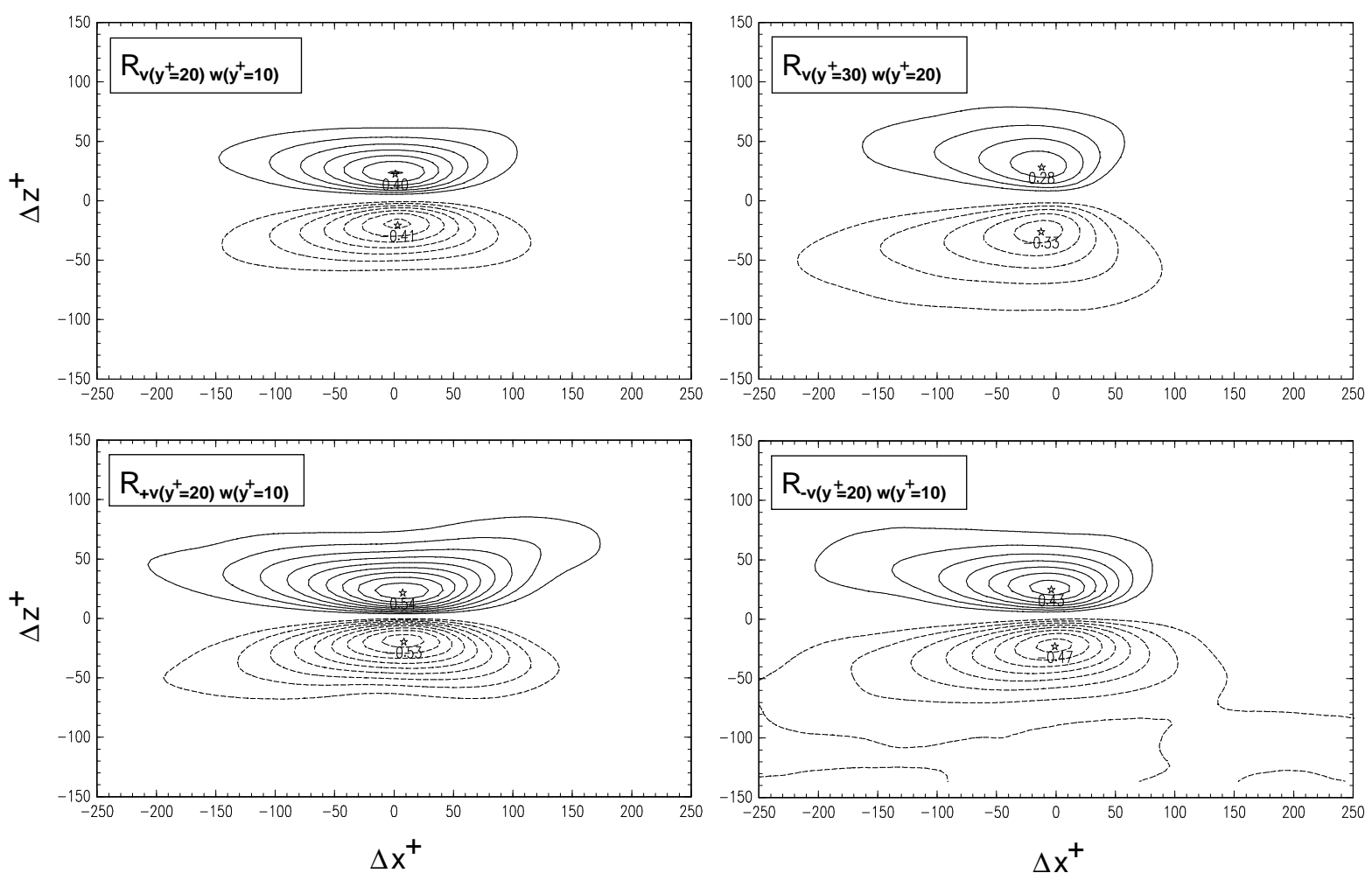

FIGURE 6.16: Top: $\quad R_{v\left(y^{+}=20\right) w\left(y^{+}=10\right)} \quad$ (left) and $R_{v\left(y^{+}=30\right) w\left(y^{+}=20\right)}$ (right). Bottom: $R_{v\left(y^{+}=20\right) w\left(y^{+}=10\right)}$ with $u<0$ and $v>0($ left $)$, and $u>0$ and $v<0$ (right) measured at $\operatorname{Re}_{\theta}=7800$. 
Figure 6.15 and figure 6.16 present the cross-correlations where the span-wise velocity component is involved. The similar shape of the correlations is clearly visible, but the larger extent of the $R_{u w}$ function with respect to $R_{v w}$ should be noted and the difference in the maximum of correlation which indicates that the $R_{v w}$ correlation exceeds the coherence of the $R_{u w}$ function. This is fully in accordance with the analysis in the previous section. Of fundamental importance are the differences in the fine structure if the correlation is considered as a function of the temporal delay and the structure dependent convection velocity highlighted quantitatively in figure 6.17 to figure 6.19. Only a line of the data extracted from the two-dimensional correlation functions along the $x$-coordinate through the maximum of correlation is shown here.
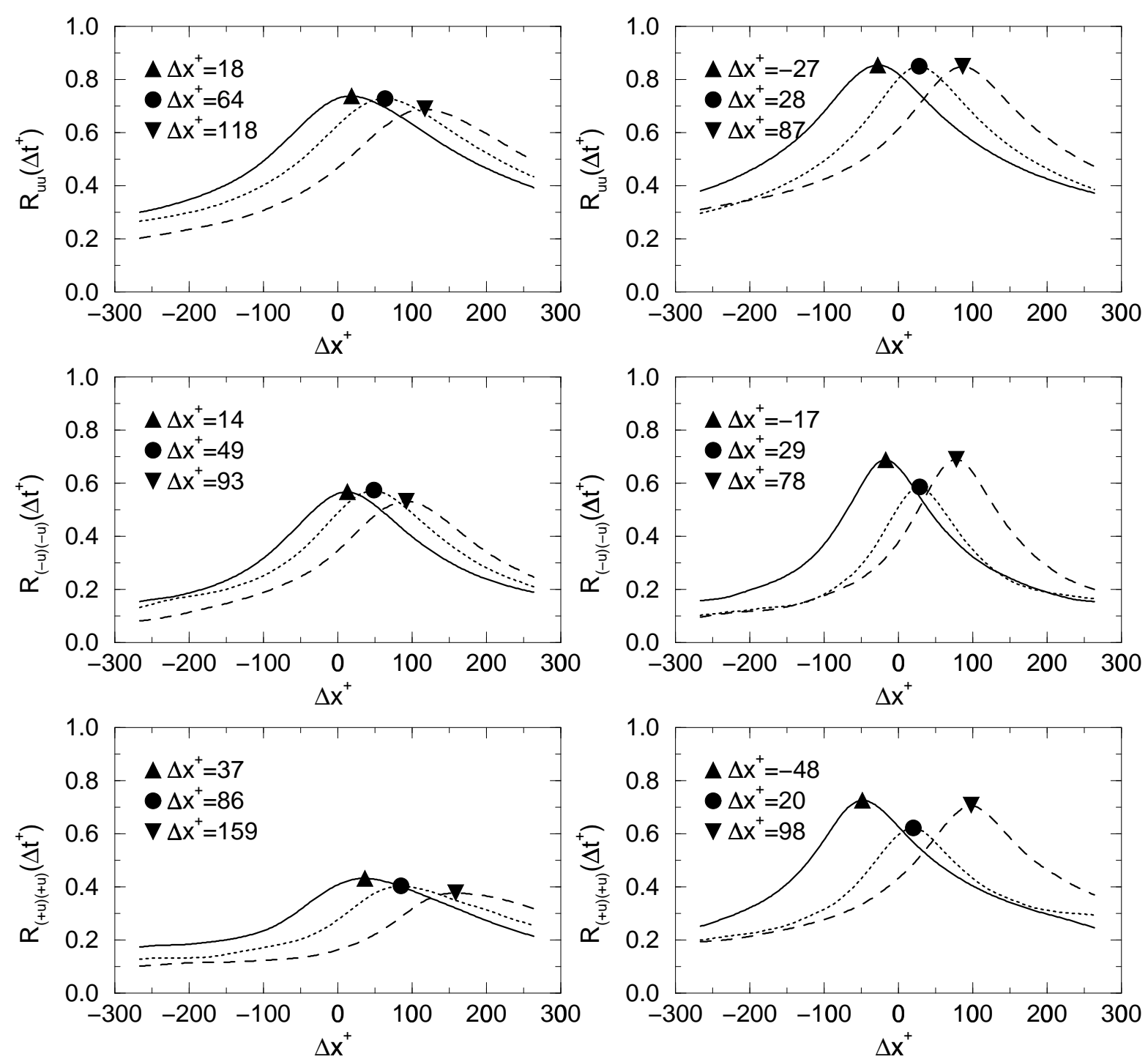

FIGURE 6.17: One-dimensional spatio-temporal correlation function of stream-wise velocity fluctuation $R_{u u}$ (top), the low-momentum fluctuations in stream-wise direction $R_{-u-u}$ (centre) and the high-momentum stream-wise fluctuations $R_{+u+u}$ (bottom) measured at $y^{+}=10$ (left column) and $y^{+}=20$ (right column) and different time delays between a pair of measurements $\left(\Delta t^{+}=-5\right.$ solid line, $\Delta t^{+}=0$ dotted line, $\Delta t^{+}=+5$ dashed line). The symbol indicates the maximum of correlation and the legend shows the exact position in wall-units. 
First of all it can be seen that the shift of the maximum (indicated by the symbols and the legend) strongly depends on the sign of the stream-wise velocity fluctuation considered for the calculation of the conditional correlation and from the wall-distance for all correlations. This becomes clear as structures whose velocity is larger than the mean velocity are travelling faster. Secondly, it turns out that the height of the conditional correlations is always lower than the correlations based on all fluctuations. Finally, it should be noted by comparing the values in the legend that the movement of high-speed structures towards and away from the wall differs to a large extent. Since the distance between each of a pair of measurement planes was 10 wall-units, it is possible to obtain information about the extension of the structures
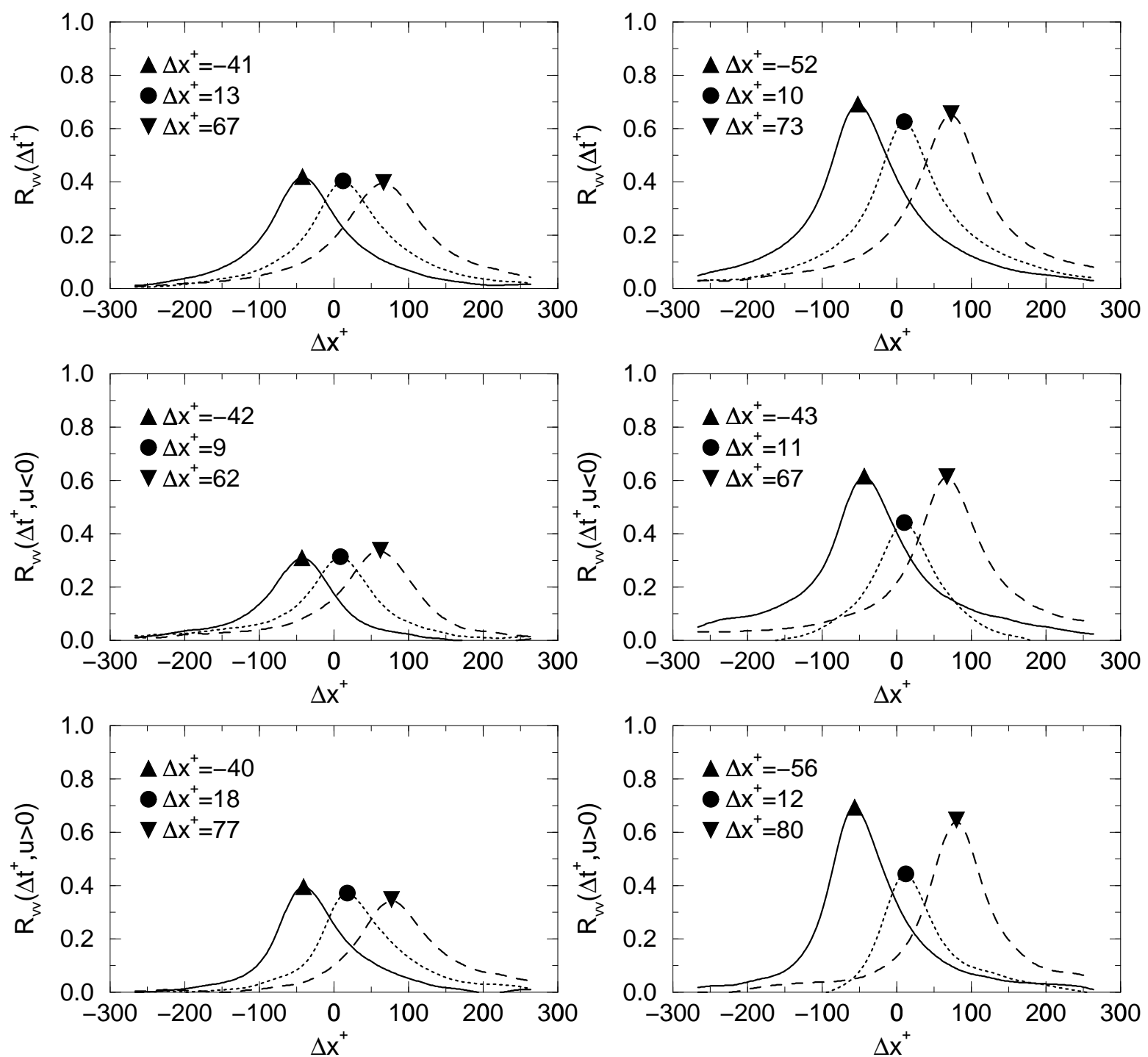

FIGURE 6.18: One-dimensional spatio-temporal correlation function of wall-normal velocity fluctuation $R_{v v}$ (top), and correlations of wall-normal fluctuation extracted in regions with $u<0$ (centre) and $u>0$ (bottom) measured at $y^{+}=10$ (left column) and $y^{+}=20$ (right column) and different time delays between a pair of measurements $\left(\Delta t^{+}=-5\right.$ solid line, $\Delta t^{+}=0$ dotted line, $\Delta t^{+}=+5$ dashed line). The symbol indicates the maximum of correlation and the legend shows the exact position in wall-units. 
in wall-normal direction, if the case is considered where both measurements were performed simultaneously. The angle $\alpha_{1}$ indicates the inclination of the dominant flow structures between $y^{+} \approx 10-20$ and $\alpha_{2}$ between $y^{+} \approx 20-30$. The angles calculated from the primary correlations are in good agreement with the observation of other authors. However, when the wall-normal extent of flow structures which are associated with the production of turbulence are considered, it turns out that the angles associated with $Q 4$ events are in general quite large with respect to the angles associated with low-speed streaks moving away from the wall. Another interesting feature is the fact that the angles increases when the wall distance becomes larger. This result can be explained by the strong velocity gradients in wall bounded flows.
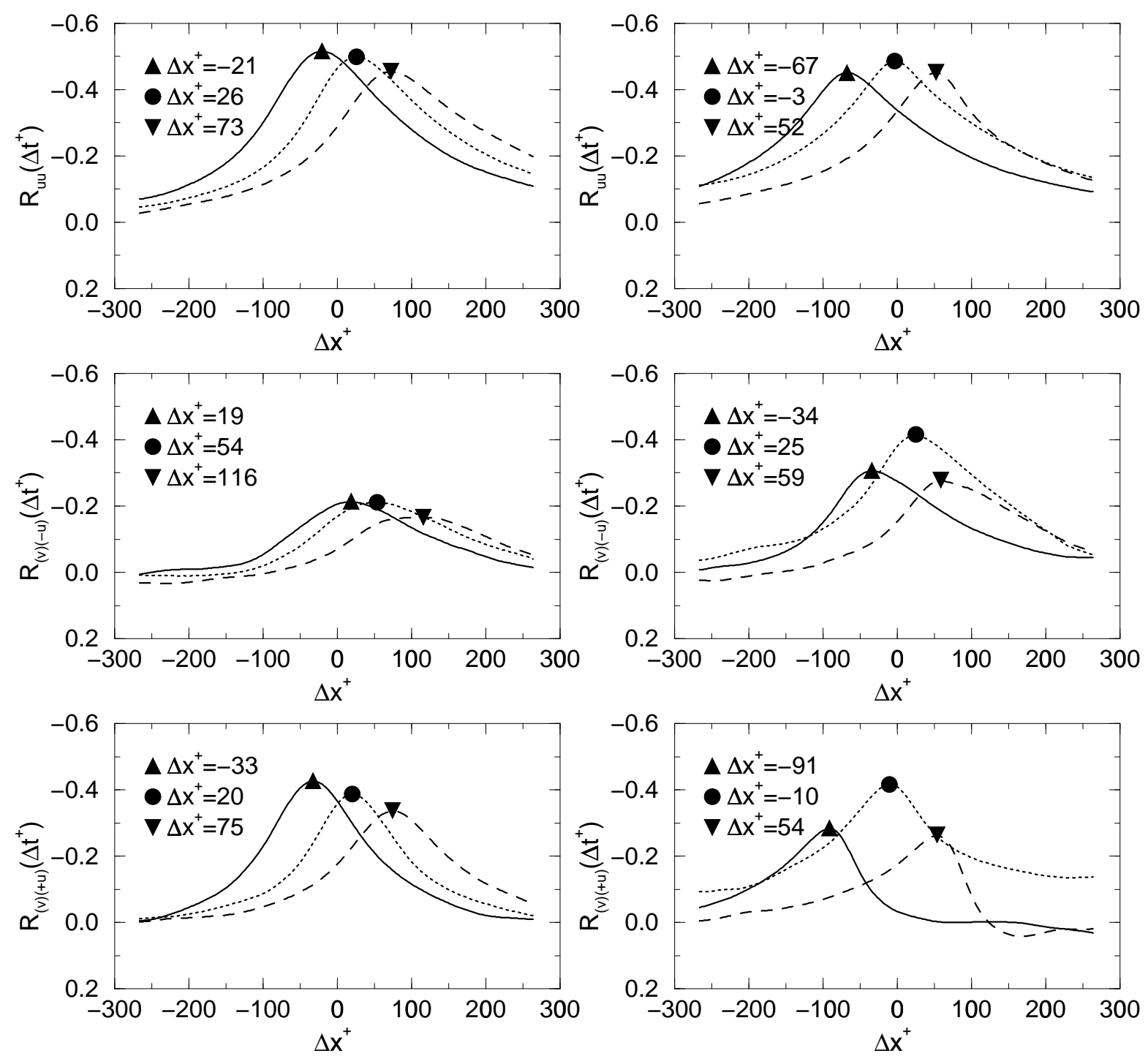

FIGURE 6.19: One-dimensional spatio-temporal cross-correlation function of stream-wise with wallnormal velocity fluctuation $R_{v u}$ (top), correlations of negative stream-wise with wall-normal fluctuation $R_{(v)(-u)}$ (centre) and correlations of positive stream-wise with wall-normal fluctuation $R_{(v)(+u)}$ (bottom) measured at $y^{+}=10$ (left column) and $y^{+}=20$ (right column) and different time delays between a pair of measurements $\left(\Delta t^{+}=-5\right.$ solid line, $\Delta t^{+}=0$ dotted line, $\Delta t^{+}=+5$ dashed line $)$. The symbol indicates the maximum of correlation and the legend shows the exact position in wall-units. 


\begin{tabular}{lcc||lc}
\hline Correlation & $\alpha_{1}[\mathrm{deg}]$ & $\alpha_{2}[\mathrm{deg}]$ & Correlation & $\alpha[\mathrm{deg}]$ \\
\hline$R_{(u)(u)}$ & 8.88 & 19.6 & $R_{(v)(u)}$ & 21.0 \\
$R_{(-u)(-u)}$ & 11.5 & 19.0 & $R_{(v)(-u)}$ & 10.5 \\
$R_{(+u)(+u)}$ & 6.63 & 26.6 & $R_{(v)(+u)}$ & 26.6 \\
\hline
\end{tabular}

TABLE 6.5: Extension of the structures in wall-normal direction

It should be emphasised that the angles, calculated here, represent the structural feature of dominant coherent velocity structures in contrast to the results presented in table 6.3 which show the average angles calculated from all structures being present in the flow.

\subsection{Properties of coherent velocity structures}

In this section the dominant coherent flow structures being present in the buffer-layer will be investigated in detail. Of primary interest is their size and shape, their significance for the production of turbulence and their mutual interaction. In the first part of the investigation flow structures will be considered which can be labelled as dominant due to their occurrence. Thereafter, flow structures are examined that can be characterised as dominant due to their large amplitude in the Reynolds stress component - $\rho u v$. It should be noted that these particular structures do not occur frequently, according to figure 6.20 where the probability density function of the Reynolds stress component $-\rho u v$ measured at $y^{+}=20$ is displayed (left). Furthermore, it can be seen from the $u v \operatorname{PDF}(u v)$ distribution in the right figure that their contribution to the total production of turbulence is quite small compared with the structures which are dominant with respect to their occurrence. However, these velocity fields represent general features which can be frequently observed even when the peak intensity of the Reynolds shear stress is usually lower.

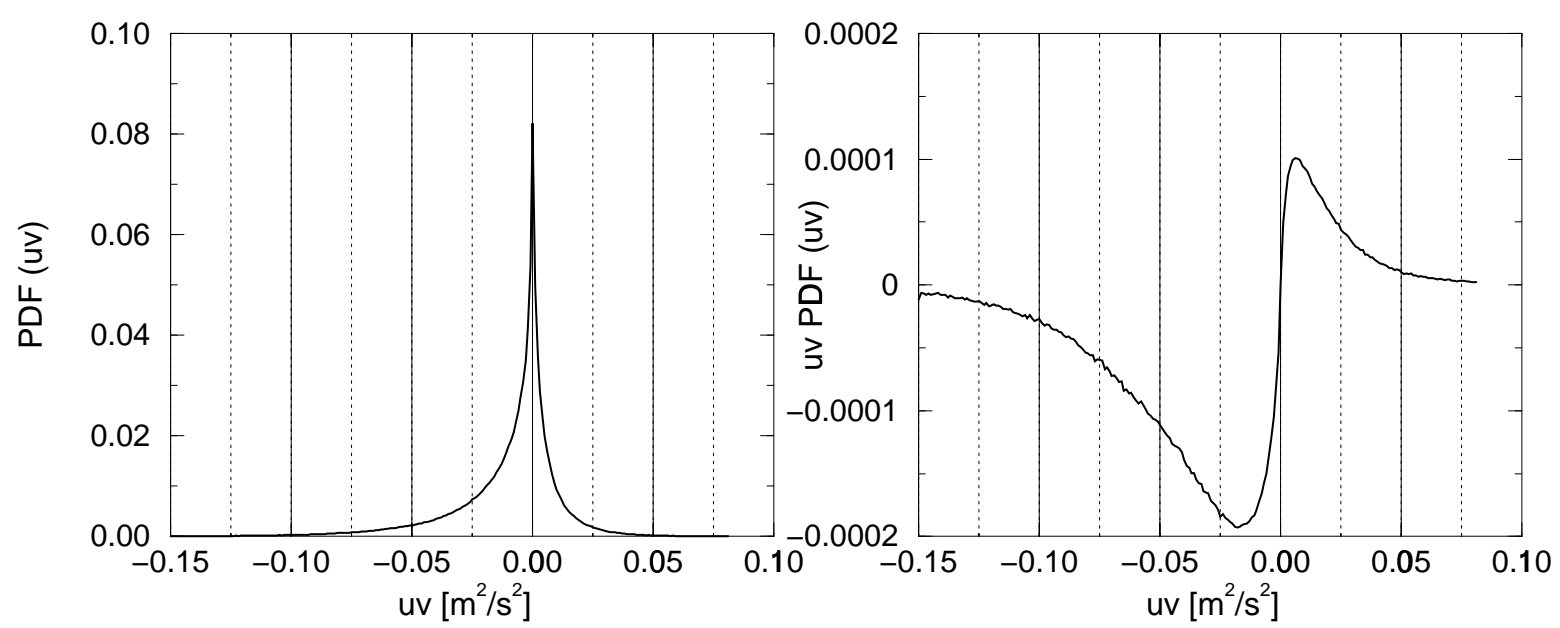

FIGURE 6.20: Distribution of the Reynolds shear stress $u v\left(y^{+}=20\right)(l e f t)$ and $u v \operatorname{PDF}(u v)(r i g h t)$.

\subsubsection{Low-speed streaks}

Low-speed streaks are the most striking structures in the near wall region between $y^{+} \approx 5$ and $y^{+} \approx 40-50$ which can be clearly observed in $x z$-planes close to the wall $[58,38,44]$. They 
appear as elongated and twisted low-speed regions, sometimes 1000 wall-units in length and on average 30 wall-units in width, with a span-wise periodicity of about 100 wall-units. The dependence on the Reynolds number according to [25] and the exact wall distance is still a point of discussion. It has been assumed that hairpin-vortices induce these low-speed region between the inclined legs while they are travelling downstream but a convincing experimental proof is still missing [46]. Some authors [7, 54] proposed that the low-speed streaks are generated between pairs of relatively weak, but highly elongated stream-wise vortices, similar to the legs of the hairpin model, but the existence of these vortices is still an open and controversial question $[94,44]$. Other authors assume that the streaks might originate from a weak vertical oscillation of the fluid layers which produces strong oscillations in stream-wise direction [66]. However, a general agreement based on the experimental and numerical results could not be achieved [14]. From flow visualisation experiments it is evident that the lowspeed streaks play a dominant role in a sequence of events referred to as bursting phenomena. Kline observed in the near-wall region of a turbulent boundary layer that extended low-speed flow structures, which move away from the wall, start to oscillate and burst finally after a certain life time into small scale turbulence [58]. The bursting of these low-speed structures may be related to an inflectional instability which is going to develop in the low-speed regions. This Kelvin-Helmholtz instability may cause an ejection of local vortices above the streaks which is associated with the production of turbulence. However, another explanation is that the ejection of low-speed fluid from the wall is associated with flow structures which transfer momentum towards the wall (sweeps or inrush bursts), located directly upstream of the region where the ejection takes place [84]. The connection between the bursting phenomenon near the wall and the large scale motion in the outer part is one of the key questions. In the vertical plane, the footprint of the sweep-streak interaction would appear as a near-wall shear-layer as discussed above, but of smaller extent in both wall-normal and stream-wise directions. From what has been said, it is obvious that the reality and relevance of the proposed models require detailed experimental information of the spatio-temporal flow structure in the near wall region.

Figure 6.21 shows two characteristic velocity fields measured in the $x z$-plane at $y^{+}=10$. The flow direction is from left to right and the local mean velocity $\bar{U}$ is subtracted from the instantaneous velocity field $U$ to display the turbulent velocity fluctuations $u=U-\bar{U}$ and $w$. Predominant structures are the elongated flow regions that convect downstream with approximately half the local mean velocity, indicated by the vectors going from right to left. The shape, extent and span-wise separation of these slightly tilted flow regions is in quantitative agreement with the literature [86], but it should be noted that the instantaneous values of the geometrical properties can deviate strongly from the averaged ones, presented in figure 6.6. The width of the structures visible in figure 6.21 for example varies between 20 and 100 wall units, but also broader streaks can be found. Another important property of the streaks is their extent in wall-normal direction as the statistical variation of their height is responsible for the increasing separation on average between the streaks with increasing wall distance. This can be concluded from the velocity fields in figure 6.22 which were measured simultaneously with the vector fields presented in figure 6.21 but at $y^{+}=20$. First of all, it is obvious that the strong variation of the streak-width vanishes. Whereas the small ones in both figures conserve their geometrical properties to a large extent, the width of the streak located at $z^{+} \approx 150$ in the top image becomes smaller with increasing wall distance. This is in agreement with the results presented in 6.7. The lower image on the other hand nicely shows that the length of these flow regions seems to decrease as well with increasing wall distance. However, as the 

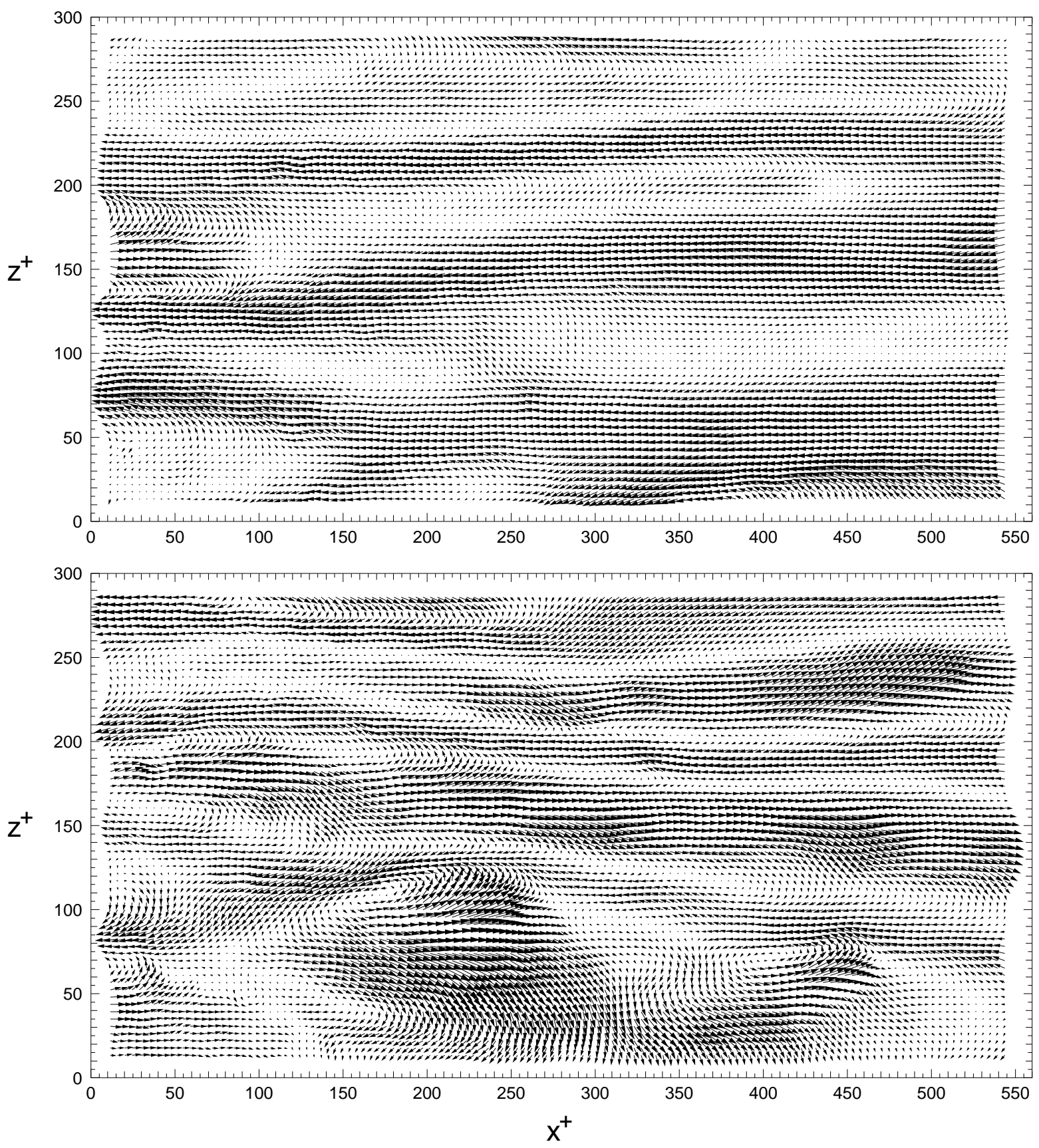

FIGURE 6.21: Velocity fluctuations measured at $y^{+}=10$.

streaks which appear separated in stream-wise direction at $y^{+}=20$ belong to the same streak visible at $y^{+}=10$, the decreasing length of these structures can be considered as an artefact related to the statistical variation of their height. When the dynamic of the streaks is investigated, it turns out that these structures tend to move away from the wall, which can be deduced from the out-of-plane motion (blue contours denote a motion away from the wall $v>0$ and red towards the wall $v<0$ ). It is clear that this process is associated with the production of turbulence according to the Reynolds equation as $\tau_{\text {turb }}=-\rho \overline{u v}$ becomes positive on average. However it is important to note the extent of this vertical motion is usually significantly shorter than the total length of the low-speed streaks at the same $y$-value. This is in agreement with the 

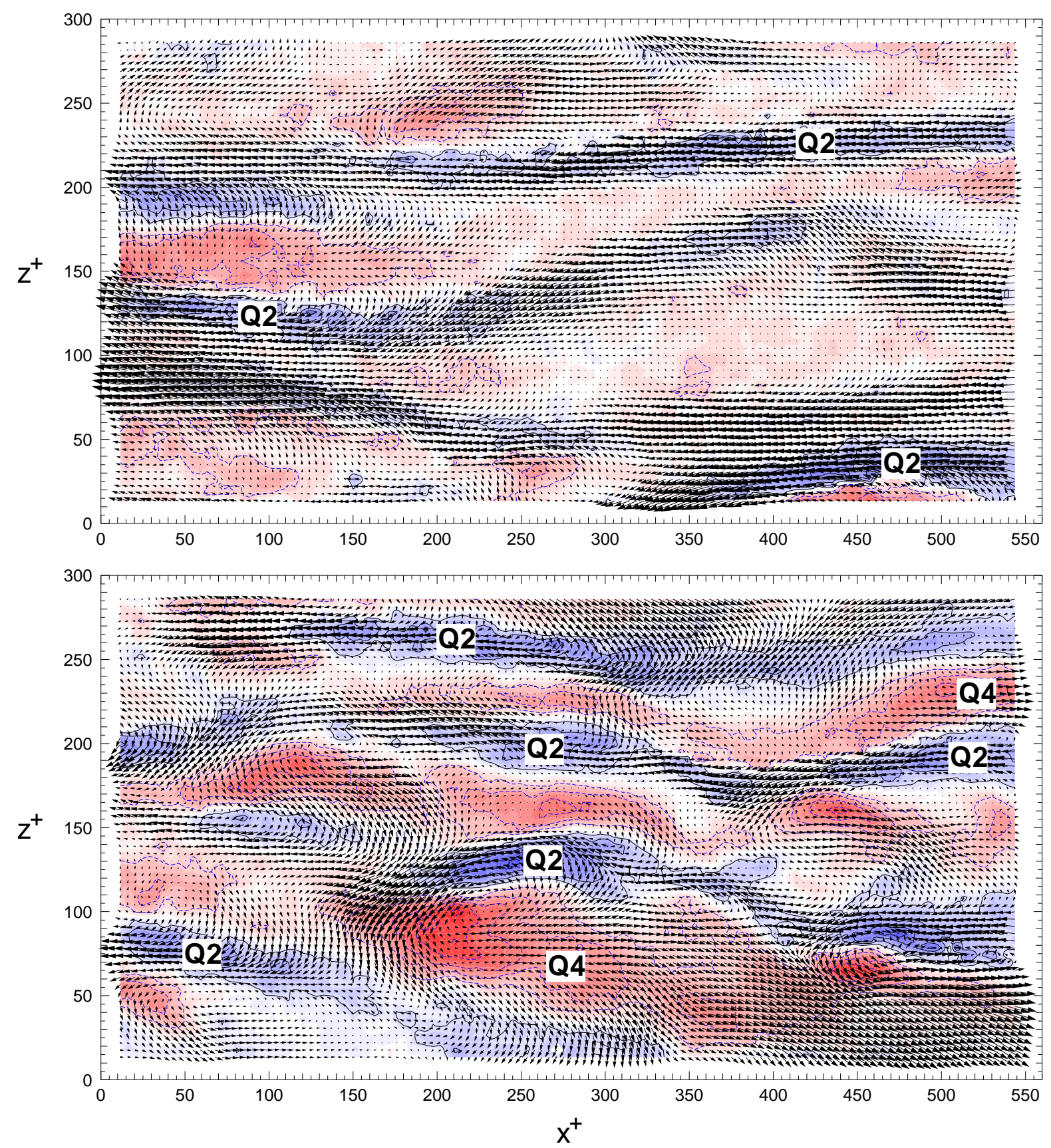

FIGURE 6.22: Velocity fluctuations measured at $y^{+}=20$ (red: $v<0$; blue: $v>0$ ).

$R_{u v}$ correlation in figure 6.8 and 6.9 and implies that no pairs of stream-wise counter-rotating vortices flank the low-speed regions over their total length, as proposed by some authors [54]. Otherwise, one would expect to detect a sign change in the out-of-plane motion on both sides of the streaks or at least a large $v$ variation over the length of the streaks.

\subsubsection{Sweeps}

The production of turbulence caused by the lifting of the low-speed streaks is one of the basic processes identified in near-wall turbulence as already mentioned. However, due to the 

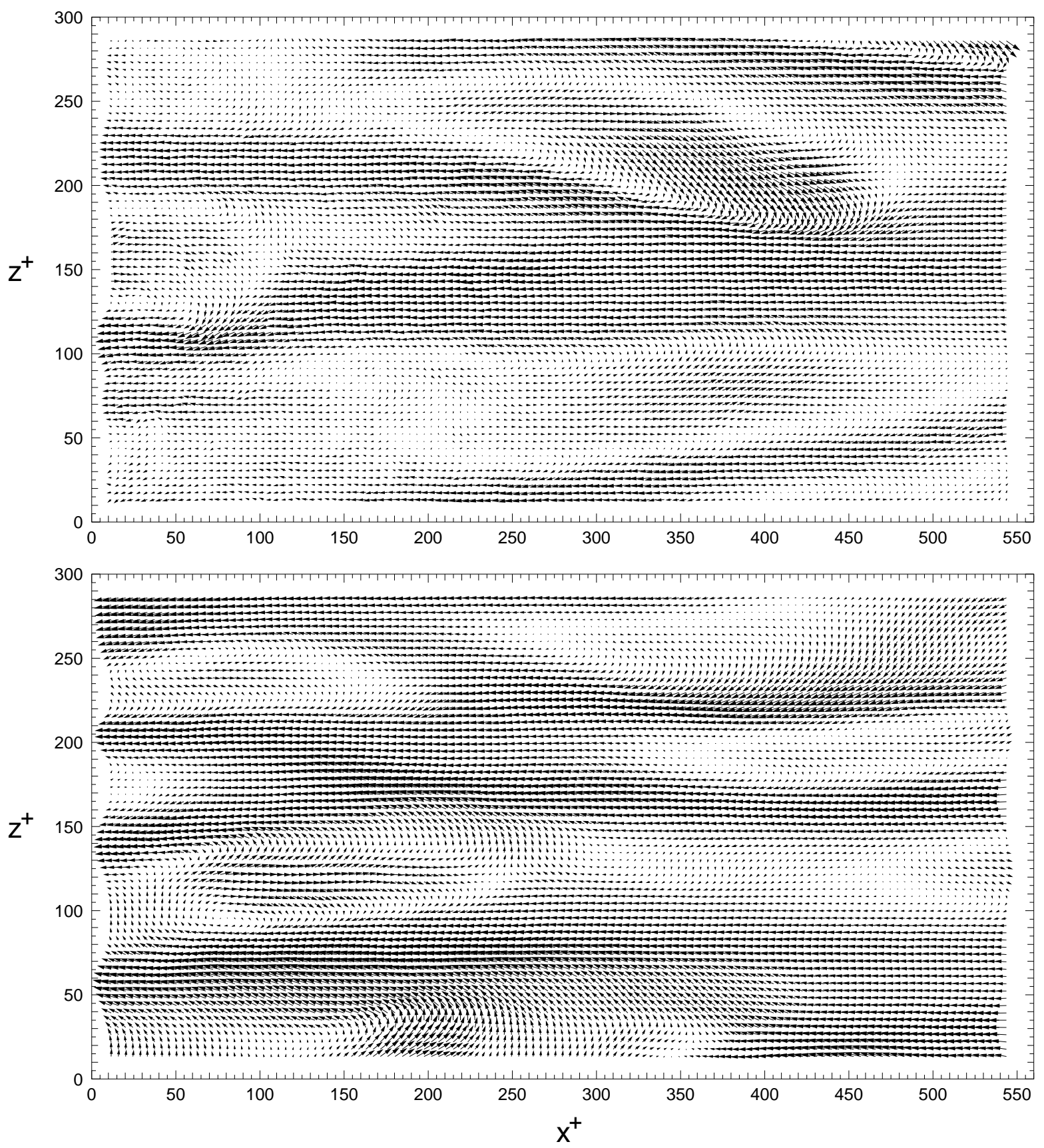

FIGURE 6.23: Velocity fluctuations measured at $y^{+}=10$.

complexity of the turbulent motion and the limitations of the measurement techniques the cause and dynamic is still the subject of controversial discussions. In order to investigate this exchange processes in detail, the interaction of the streaks with the surrounding fluid will be investigated next. Especially the interaction regarding the high momentum flow structures which move towards the wall will be considered, as only these possess the energy required to alter the turbulence structure of the flow field effectively.

It is evident that the high-momentum flow structures must be visible in nearly each velocity field in order to compensate the low-momentum movement of the streaks, but in contrast to the low-speed streaks the variety of these structures is much larger. Usually they appear 

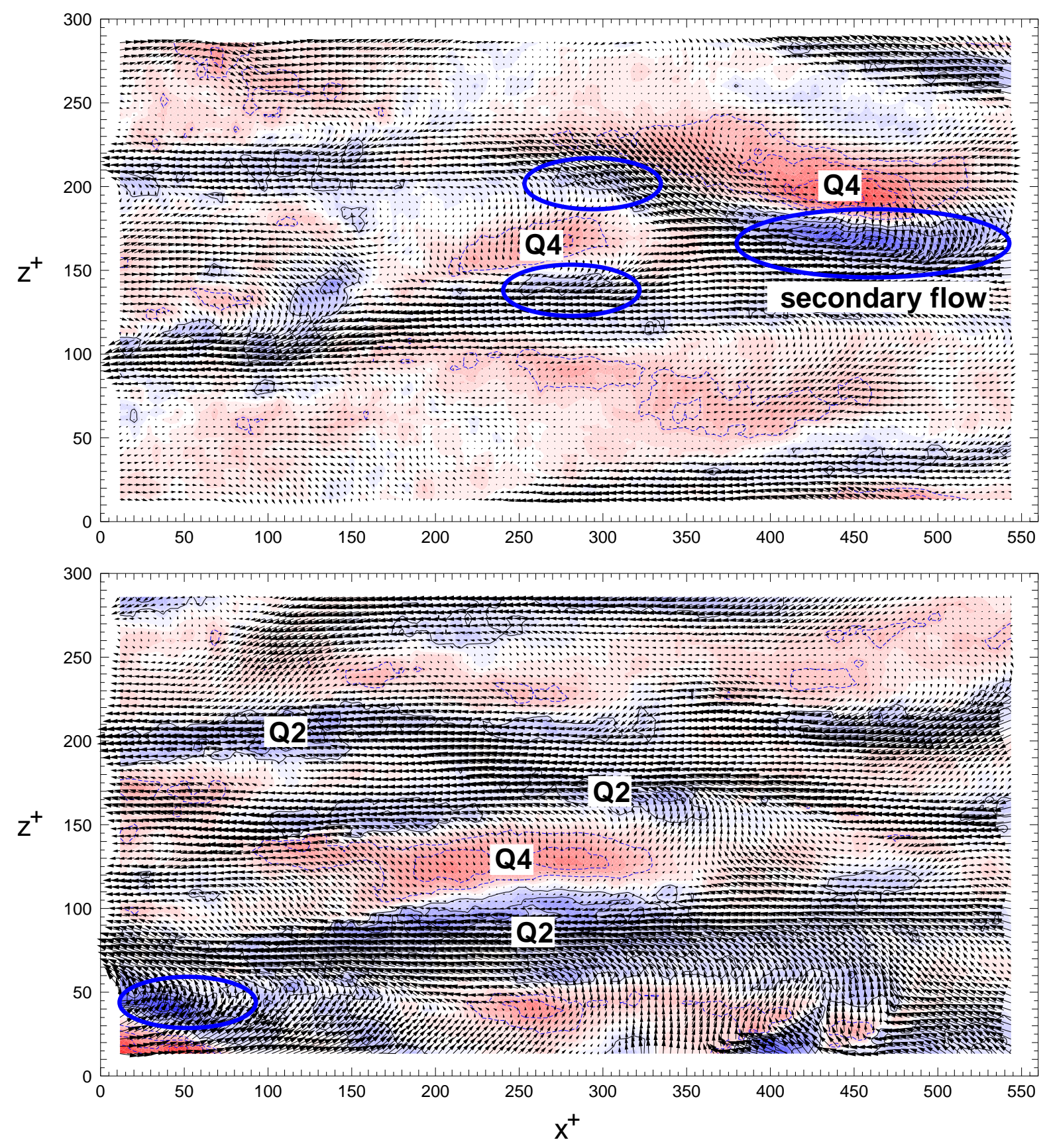

FIGURE 6.24: Same as figure 6.23 but measured at $y^{+}=20$ (red: $v<0$; blue: $v>0$ ).

less elongated and broader than the low-speed regions because these flow structures originate statistically from flow regions which are further away from the wall, see section 5.4. This can be estimated from the sign of the wall-normal velocity component which is represented by the contours in figure 6.22. At $y^{+} \approx 10$ these high momentum structures look sometimes similar to the streaks with regard to size and shape according to figure 6.21 but more frequently they resemble small elliptically shaped islands, approximately 200 wall units in length and roughly 50 to 100 wall-units in width according to figure 6.23. It can be seen from the out-of-plane velocity component, displayed in figure 6.24, that these structures, which are labelled as sweeps, transfer momentum towards the wall. Thus, these structures are statistically represented by 

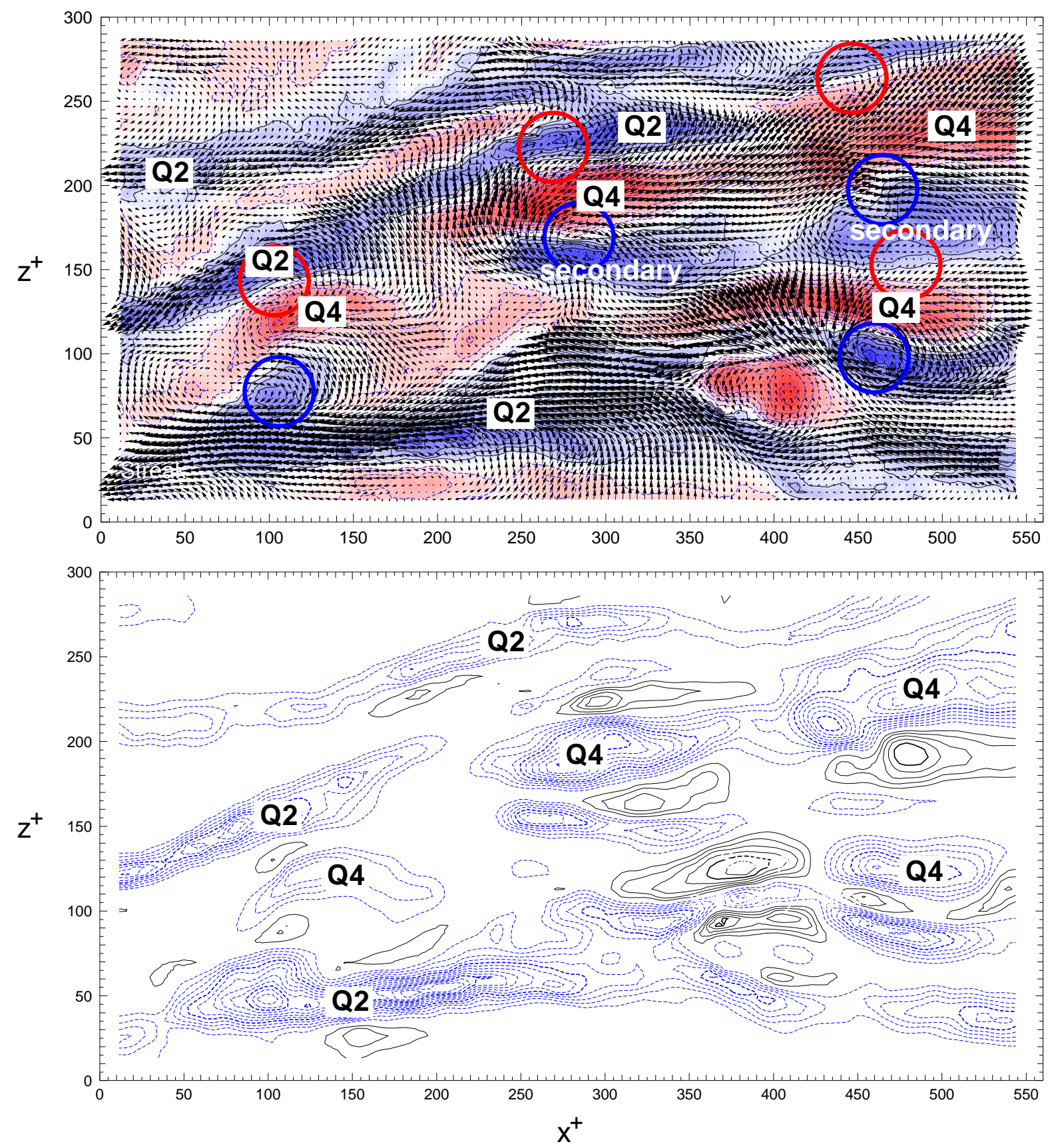

FIGURE 6.25: Velocity fluctuations measured at $y^{+}=20$ (top) and Reynolds stress component $u v$ (bottom).

the correlations shown in figure 6.8. If such a structure moves towards a low-speed streak, as visible in figure 6.24 for example, an interaction takes place and parts of the streaks, which are directly affected by the sweeps, are forced to move away from the wall due to continuity (see blue region in ellipse). On average, this process is represented by the spatial correlation functions shown in figure 6.10 to 6.12. However, it can be seen from the samples shown in figure 6.24 that the effect on the streaks is quite small in the present case. This can be explained by the fact that the momentum is insufficient to create a strong lifting of the streak. Figure 6.25 shows the same interaction but the momentum transferred by the sweeps is much larger. In 

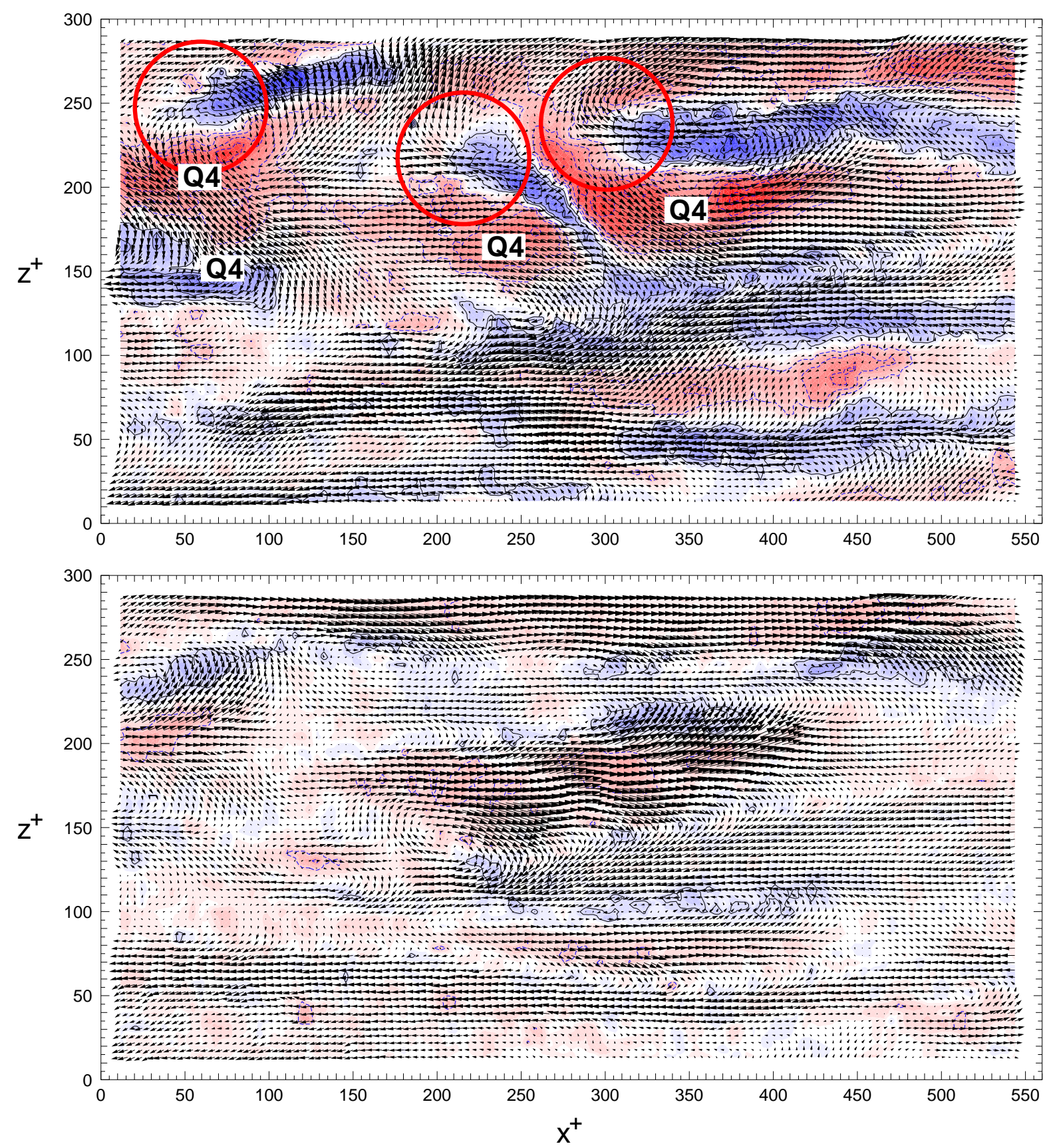

FIGURE 6.26: Velocity fluctuations measured simultaneously at $y^{+}=20$ (top) and $y^{+}=10$.

effect, the streaks move away from the wall, indicated by the blue contours, and it is visible from the lower image that this is associated with the production of Reynolds shear-stress (dashed lines indicate $u v<0$ ). Though, it should be noted that the production of Reynolds shear stress induced by the sweeps is quite large as can be estimated from regions denoted by Q4 in the lower contour plot. Based on this experimental result it can be concluded that, in general, the lifting of low-speed streaks can be considered as a secondary motion which is induced by an interaction between a low-speed streak with a sweep, and the size and strength of the region, which moves away from the wall, is related to the momentum of the sweep. In addition it can be stated that the lifting of low-speed fluid into higher momentum flow regions 


\subsection{Properties of coherent velocity structures}

is accompanied by two weak stream-wise vortices because any local motion away from the wall is associated with a stream-wise vortex pair, but these vortices are produced locally and can not be considered as primary vortex structures. The similar stream-wise extent of sweeps and regions of significant outward motion supports this conjecture. The average length of these vortices can be estimated from figure 6.10 to 6.12 .

Another effect associated with the movement of the sweeps is the creation of counter rotating vortex pairs, as indicated by the red and blue circles in figure 6.25. First of all, it should be noted that these structures are different from the vortex models presented in the figure 1.3 as these vortical structures pump high-speed fluid towards the wall and not low-speed away as implied by the vortex models on page 6 . Besides, it seems likely that these vortices are generated when the sweeps interact directly with the low-speed regions. Another remarkable effect which can be observed quite frequently is the generation of hairpin like structures when a sweep interacts directly with the back of a streak, see red circles in figure 6.26 for example. However, it can be seen from the lower image of the same figure that these vortices do not extend into the near-wall region down to $y^{+}=10$ in the present case. It can be speculated that this might be related to the strength of the sweep or that the sweep streak interaction did not last long enough at the time the image was acquired to form a clear vortex pair.

\subsubsection{Ejection}

Figure 6.27 does not reveal any extended streak pattern but the intensity of the Reynolds stress component is very large as can be estimated from the lower image. However, by visual inspection of the velocity field it can be seen that the regions of strong production are associated with counter rotating vortex pairs, denoted by the circles, which indicate the presence of a hairpin like structures, [44]. Moreover it seems that these structures are convecting as a package as described in [112]. It should be kept in mind that according to figure 6.20 the occurrence of such arrangements of hairpin like structures is very low in the near-wall region as only a minority of the fluctuations exhibits an intensity which is typically associated with such structures. Figure 6.28 shows another pair of velocity fields measured simultaneously at $y^{+}=20$ (top) and 10. Clearly visible are the different structures discussed above and their interaction. In this representation the vortical flow structures are inclined in stream-wise direction as the structures in the lower representation appear slightly upstream with respect to the results in the upper image. Since the position and intensity may be affected by the particular convection velocity subtracted, the wall-normal vorticity component $\omega_{y}$ is shown in figure 6.29 for both cases, calculated from the instantaneous velocity fields. Whereas the shear layers beneath the streaks appear clearly in this representation, the vortices are less pronounced. However, the relatively sudden change in the vorticity component at the beginning of a streak might indicate the presence of hairpin like structures.

In the last decades of the previous century, detailed hot wire investigations were performed in order to obtain quantitative information about the bursting phenomena. By using different pattern recognition techniques, mentioned on page 90, a quite regular normalised velocity signal could be extracted and it was assumed that this pattern is the signature of a coherent structure which is associated with the bursting phenomena [102]. In the near-wall region below $y^{+}=30$ the pattern could be observed in nearly $65 \%$ of the total samples, and by analysing the individual velocity signal it was found that the length of the pattern varied over quite a wide range (1:25). To identify the structures which are responsible for the characteris- 

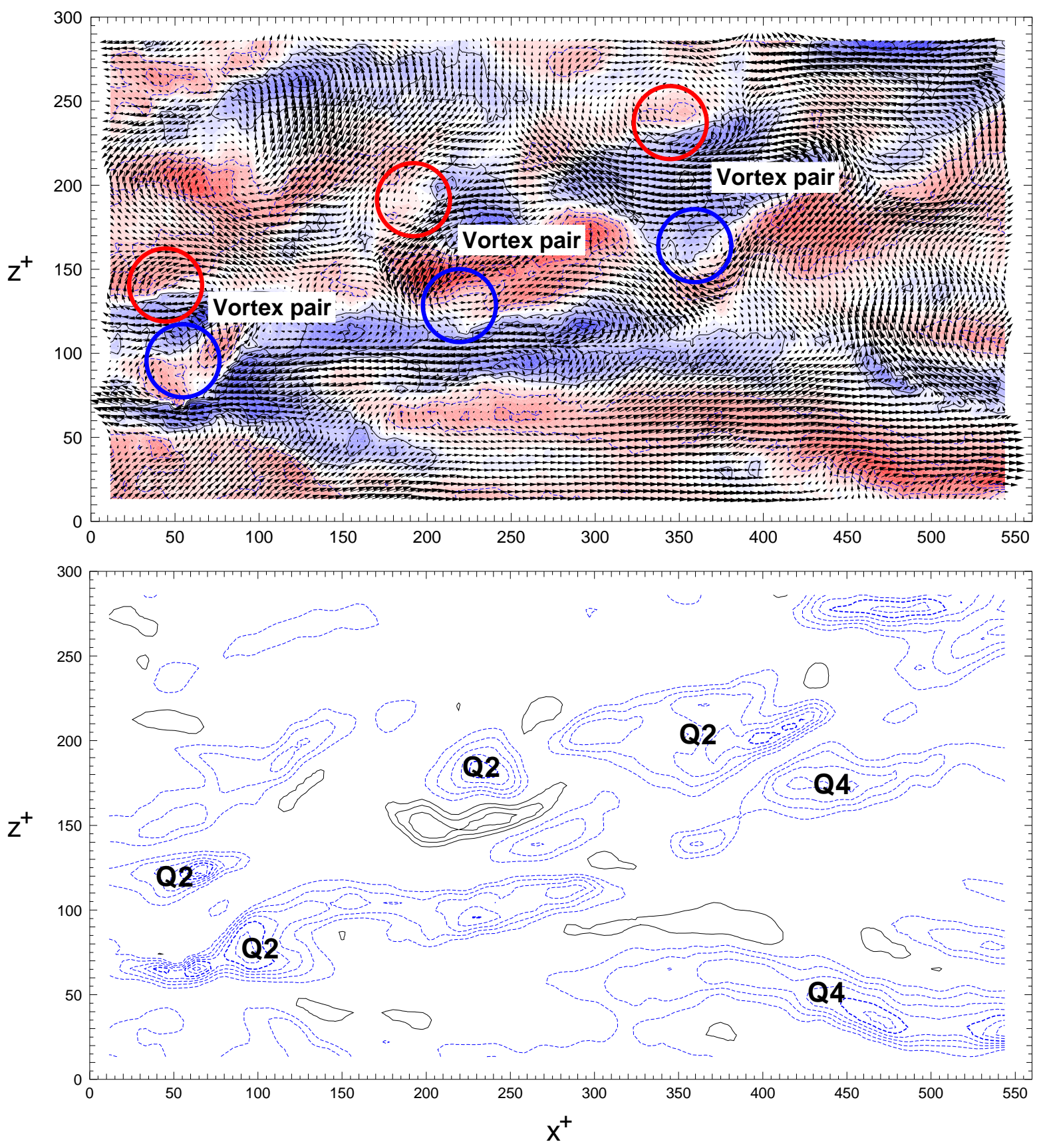

FIGURE 6.27: Velocity fluctuations measured at $y^{+}=20$ (top) and Reynolds stress component $u v$ (bottom).

tic velocity pattern, the velocity structure of the PIV measurements is analysed in stream-wise direction for various span-wise locations. For the comparison it is important to keep in mind that in the PIV experiment the spatial variation of the velocity is considered at a fixed time while the time signal of the velocity is considered at a fixed point in the hot wire investigation. The left image of figure 6.30 shows three typical instantaneous signals of the $u$-velocity component which nicely matches with the ensemble averaged pattern deduced from the hot wire measurements. The signals were extracted from figure 6.28 along the red lines located at $z^{+}=70,120$ and 220. It can be clearly seen that the characteristic velocity pattern is 

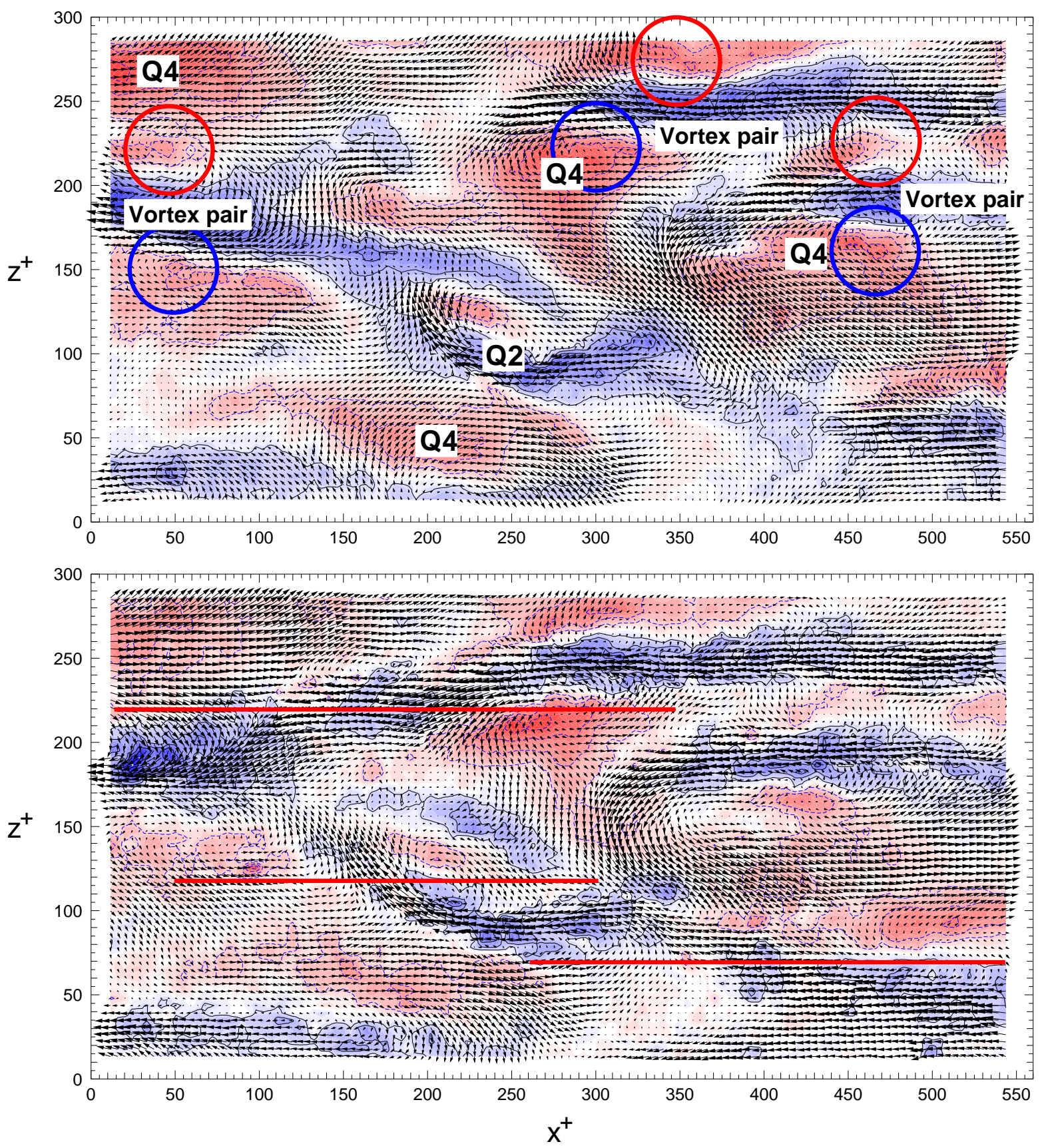

FIGURE 6.28: Velocity fluctuations measured simultaneously at $y^{+}=30$ (top) and $y^{+}=20$.

directly associated with the low-speed streaks. As these flow structures appear frequently in the near-wall region according to figure 6.21 to 6.30 , the high detection rate in the hot wire results is not surprising and also the strong variation of the pattern length can be explained. The maximum length is given by the extension of the streaks, which can be longer than 1000 wall-units, and the lower limit of the length appears when only the cross-section of a narrow streak convects along the probe. Since the streaks are only slightly twisted, the projection of the width of the cross-section in stream-wise direction is the relevant parameter for the lower limit of the pattern length. As the minimum width is approximately 30 wall units, according to section 6.2.2, and the maximum angle between the stream-wise coordinate and the streak 

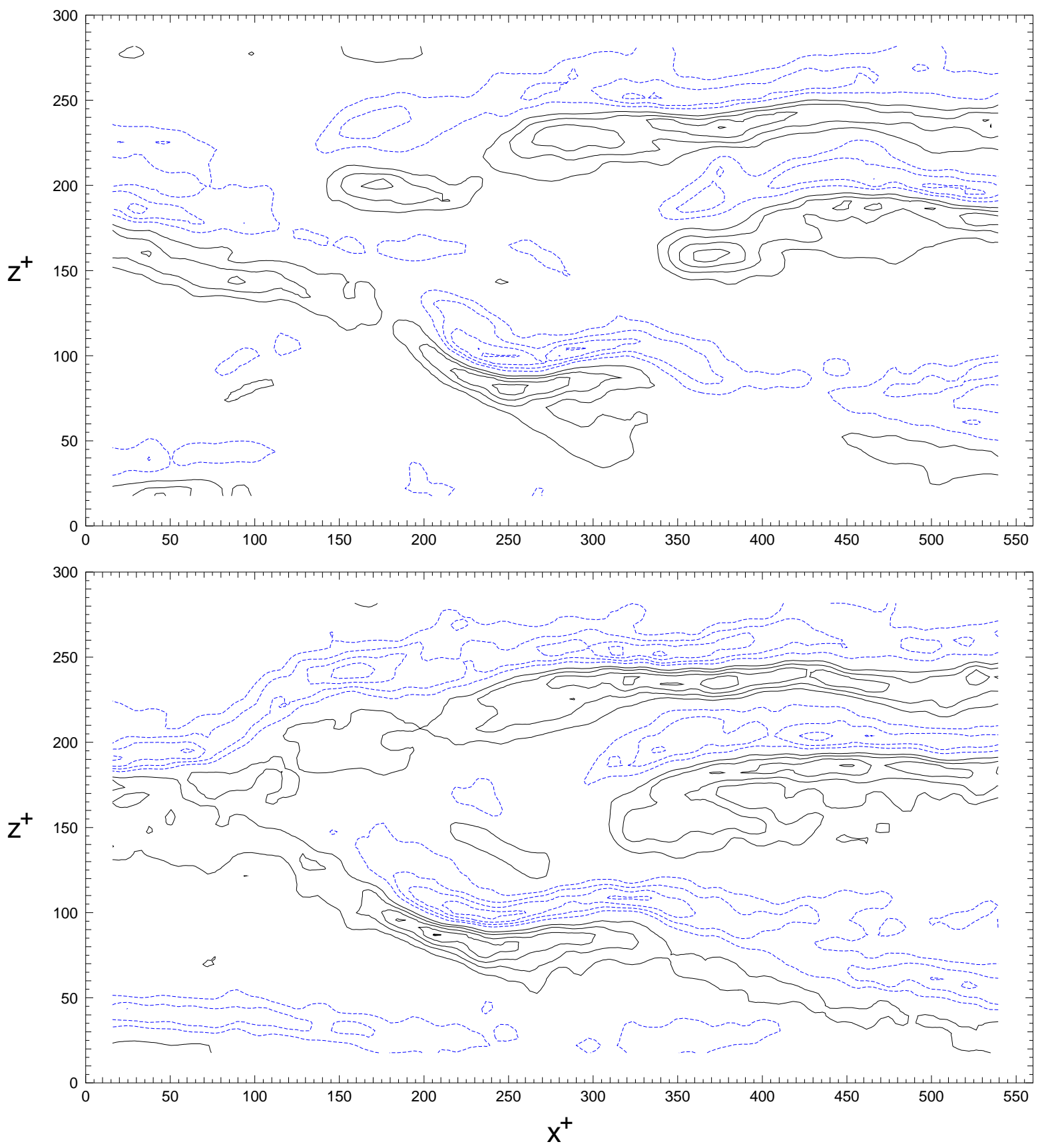

FIGURE 6.29: Vorticity fluctuation calculated from figure 6.28.

is approximately $45^{\circ}$, according to the previous figures, the projection of the cross-section in stream-wise direction is roughly 42 wall-units. Thus, the variation of the length can be estimated to $1000 / 42 \approx 24$ which nicely corresponds with the result deduced from the hot wire investigation. However, in contrast to the hot wire results, the maximum of the graphs shown in the left image of figure 6.30 always appears on the left hand side from the minimum while in the results presented in [102] the maximum appears on the right hand side. This can be explained by the different representations. While in [102] the pattern is displayed against the time and here against the spatial coordinate, a small time in [102] correspond to a large downstream position. So when the time axis of the hot wire results is reversed for comparison, the 

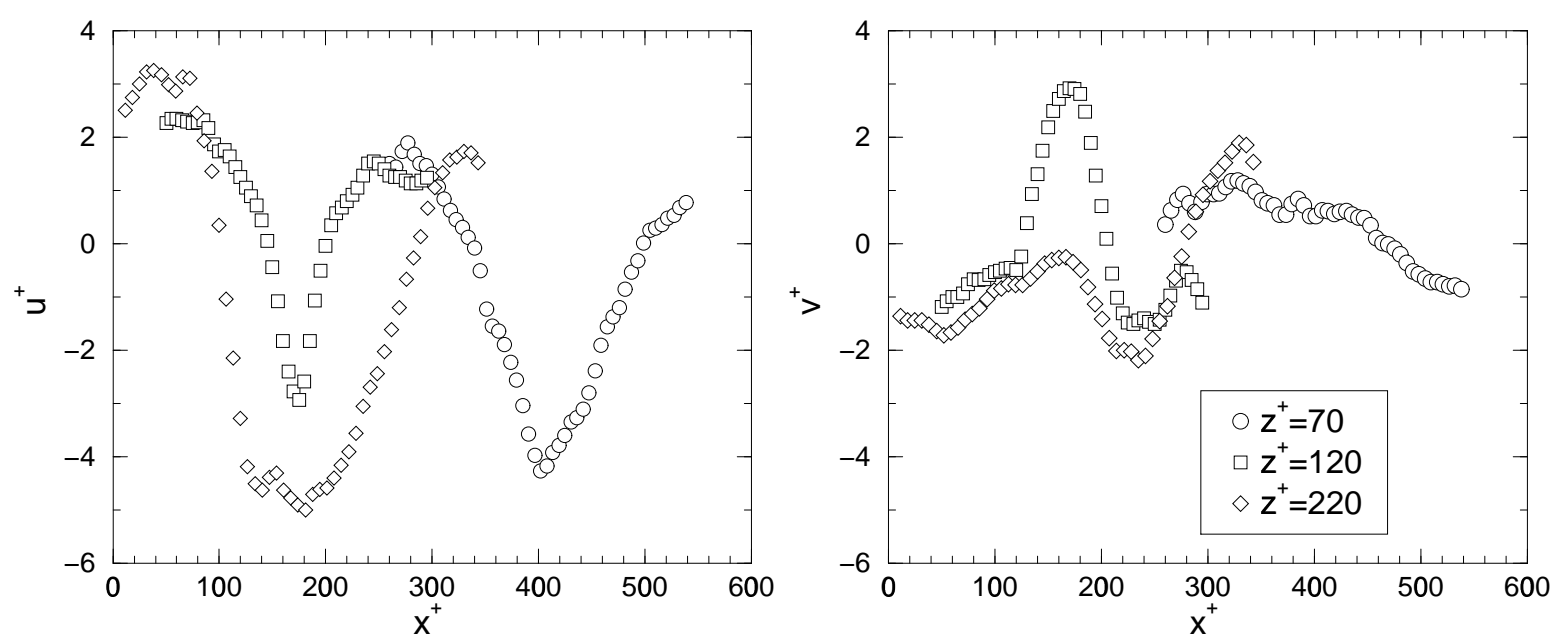

FIGURE 6.30: Profiles of $u^{+}$and $v^{+}$velocity components along red lines in figure 6.28.

functional dependence of the graphs agrees nicely. From the analysis of the velocity fields in this section, it has already been seen that the production of turbulence is quite often associated with the streaks. Nevertheless, it is obvious that the velocity pattern, shown in figure 6.30, can be only considered as a necessary condition for the production of turbulence by means of low-speed streaks. This becomes evident when the variation of the wall-normal velocity component, shown in the right image of figure 6.30, is compared with the left image. From the sign of the fluctuations it can be estimated that a significant amount of turbulence is only produced in case of the graphs with the circular and squared symbols as only in this case the wall-normal velocity component is positive while the stream-wise velocity is negative. At the end of this chapter it should be mentioned that the interpretation of the results presented in figure 6.30 requires that the flow structures keep their spatial organisation while travelling down-stream. The validity of this assumption, which is called frozen pattern hypothesis, will be proven in the next chapter. 
6 Investigation of the $x z$-plane 


\section{Investigation of the yz-plane}

The interpretation of the results presented in figure 6.30 on page 133 was based on the assumption that the spatial variation of the instantaneous turbulent velocity signal $u(x, y)$ can be transformed into a time dependent velocity signal $u(y, t)$ at a fixed point according to

$$
\frac{\partial u}{\partial x}=-\frac{1}{\bar{U}} \frac{\partial u}{\partial t}
$$

It is obvious that this so called Taylor hypothesis is certainly valid when the local convection velocity $\bar{U}$ is large relative to the turbulent fluctuations $u$. However, the statistical results shown in figure 5.3 on page 77 indicate that the maximum of the non-dimensional streamwise velocity fluctuation is around 3 at $y^{+} \approx 15$ and the average velocity is roughly 10 at the same wall location according to figure 5.2. Although the ratio between both values is only 0.3 at the measurement location where figure 6.30 was recorded, further evidence is required to justify the assumption made because it is not evident that $\bar{U}$ is the correct convection velocity of the flow structures. For this reason the dependency of the spatio-temporal correlations of the velocity fluctuations will be investigated in this chapter. In addition, various spatial correlation and cross-correlation functions of the velocity fluctuations will be investigated to validate the concepts proposed in chapter 6 to explain the turbulent mixing by means of coherent structures. Furthermore the dimensions of the shear-layers in the $y z$-plane will be considered and the characteristic features of the stream-wise vortices will be examined as well as their significance for the turbulent mixing. This is of great interest as pointed out in the introduction, because it is generally assumed that these vortices play a dominant role for the momentum exchange in wall bounded flows, as indicated in figure 1.3. This work can be seen as a completion of the conventional PIV investigation described in [13] and the flow visualisation described in [28]. Here only the main results will be analysed in detail. The additional information presented in the following may serve for comparison with the predictions of fundamental turbulence models and for the validation of numerical flow simulations in the future.

\subsection{Experimental set-up}

The multiplane stereo PIV technique in the configuration utilised for this investigation consists of four pulsed Nd:YAG lasers (BMI) each with an output energy of $255 \mathrm{~mJ}$ per pulse at $\lambda=532$ $\mathrm{nm}$, two optical benches for the generation of independent light-sheets (one for each state of polarisation), and four Peltier cooled high resolution cameras (PCO) with 1280 by 1024 pixel resolution and 12 Bit dynamic range. The schematic arrangement of the equipment with respect to the test-section is outlined in figure 7.1. This arrangement follows directly from the experimental setup, described in section 6.1, after rotating the orientation of the 
light-sheet by turning the cylindrical lens in the light-sheet optic. The observation angle for each camera in angular imaging configuration with Scheimpflug correction is shown in the following table along with the exact positions of the master cameras ( 1 and 2 in figure 7.1) with respect to the centre of the field of view (all asymmetries have been taken into account for the calculation of the three velocity components). Four $180 \mathrm{~mm}$ lenses (Carl Zeiss)

\begin{tabular}{ccccc}
\hline camera & $x[\mathrm{~mm}]$ & $z[\mathrm{~mm}]$ & $d_{o}[\mathrm{~mm}]$ & $\alpha[\mathrm{deg}]$ \\
\hline 1 & -1570 & 1464 & 2143 & 42.9 \\
2 & -1560 & -1463 & 2140 & 43.2 \\
\hline
\end{tabular}

TABLE 7.1: Position and observation distance of the master cameras with respect to the centre of each field of view (meeting point of optical axis) and corresponding observation angles.

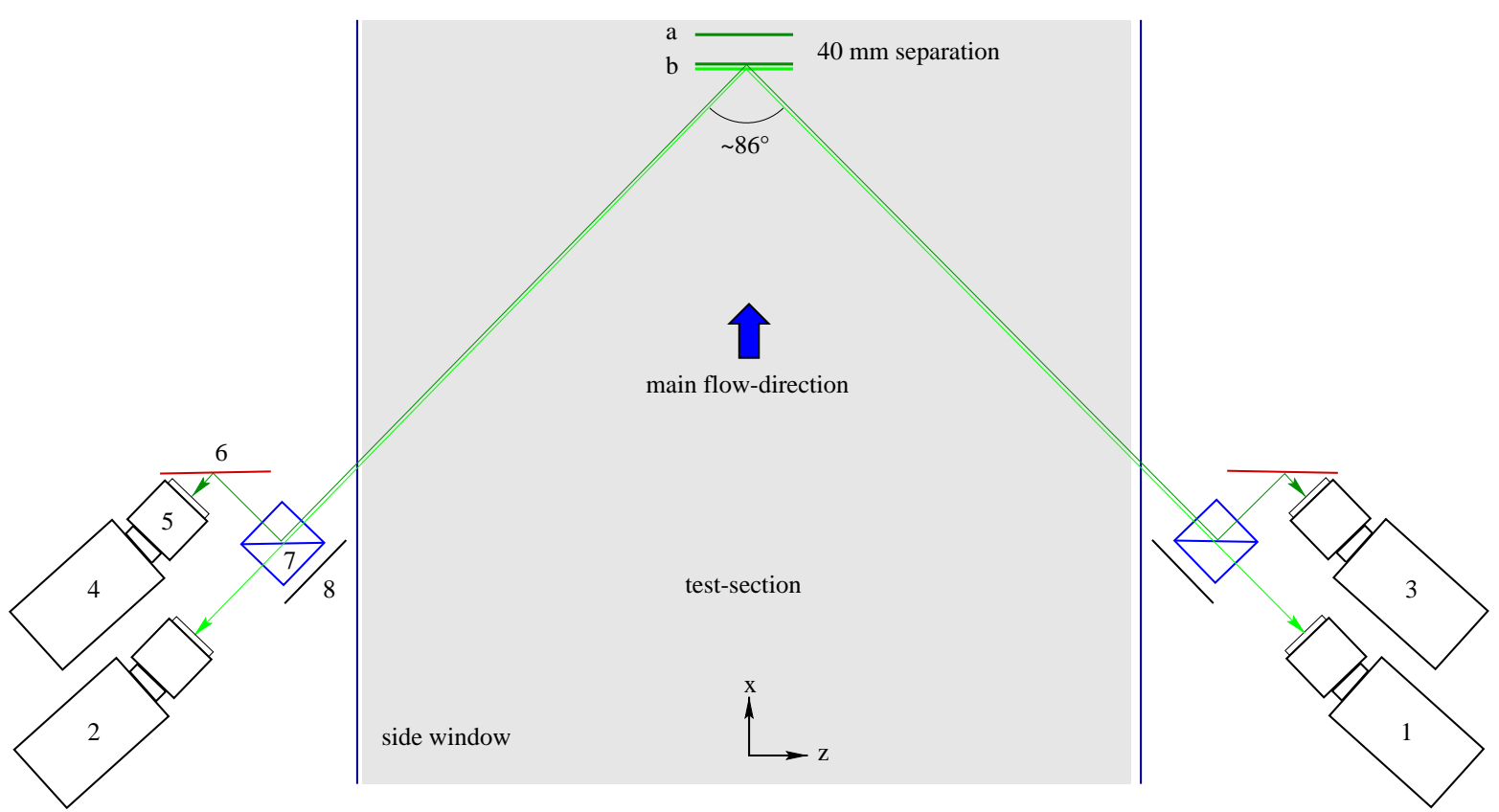

FIGURE 7.1: Schematic set-up of the recording system and light-sheet position for both experiments (different scales). 1-4 digital cameras, 5 lens, 6 mirror, 7 polarising beam-splitter cube, 8 absorbing material, a measurement location for 1st investigation (40 $\mathrm{mm}$ separation between both measurement planes in stream-wise direction), $\mathbf{b}$ measurement location for 2 nd investigation (all measurement positions at the same location). Different light ray colours indicate different states of polarisation.

were used for the measurements with an aperture of 8 and a magnification of $1 / 6$ along the principal axis of the lens. Due to the strong out-of-plane velocity component, as a result of the light-sheet orientation relative to the main flow direction, the light-sheet pairs with equal polarisation have been shifted in stream-wise direction as indicated in figure 7.2. This improves the signal to noise ratio, as the loss-of-correlation due to unpaired particle images is minimised without reducing the dynamic range and the spatial resolution. Decreasing $\Delta \tau$ or increasing the magnification of the imaging system would reduce the dynamic range or spatial resolution. 
For the evaluation of the stereo-scopic images the second order warping technique was applied again, along with the calibration validation procedure described in section 3.3. This ensures that the interrogation spots from each of a pair of stereo-scopic images correspond to the same region of the flow. The interrogation of the data was performed with the FFTbased free shape cross-correlation outlined in section 2.4, and for the determination of the signal-peak with sub-pixel accuracy, the two-dimensional Gaussian fit using the LevenbergMarquardt method has been applied. This peak finding method is less sensitive to sub-pixel displacements compared with the three point Gaussian peak fit, see figure 2.14 and table 2.1 for details. For the calculation of the velocity vectors $32 \times 32$ pixel interrogation windows were used for both Reynolds number investigations. The bandwidth of particle images displacements varies between zero and 8 pixel (zero and -8 for the left camera system in figure 7.1) for the light pulse delay listed in table 7.2, and the number of spurious vectors was on average below $0.1 \%$ by applying the following set of band-pass and gradient filter $(-3<\Delta z<10$ pixel; $-5<\Delta y<5$ pixel and $\Delta x_{i}-\Delta x_{i+1}<4$ pixel). The basic details about the recording and evaluation are summarised in table 7.2.

\begin{tabular}{lrrl}
\hline$R e_{\theta}$ & 7800 & 15000 & {$[1]$} \\
$R e_{\delta}$ & 74000 & 160000 & {$[1]$} \\
$R e_{x}$ & $3.6 \times 10^{6}$ & $8.4 \times 10^{6}$ & {$[1]$} \\
\hline$U_{\infty}$ & 3 & 7 & {$[\mathrm{~m} / \mathrm{s}]$} \\
$u_{\tau}$ & 0.121 & 0.263 & {$[\mathrm{~m} / \mathrm{s}]$} \\
$\delta$ & 0.37 & 0.34 & {$[\mathrm{~m}]$} \\
$\delta^{+}$ & 3000 & 5980 & {$[1]$} \\
field of view & $63 \times 98$ & $63 \times 98$ & {$\left[\mathrm{~mm}^{2}\right]$} \\
field of view & $0.17 \times 0.26$ & $0.19 \times 0.29$ & {$\left[\delta^{2}\right]$} \\
field of view & $511 \times 796$ & $1107 \times 1722$ & {$\left[\Delta y^{+} \times \Delta z^{+}\right]$} \\
spatial resolution & $0.6 \times 2.13 \times 2.13$ & $2.13 \times 2.13$ & {$\left[\mathrm{~mm}^{3}\right]$} \\
spatial resolution & $5.0 \times 17.3 \times 17.3$ & $37.4 \times 37.4$ & {$\left[\Delta x^{+} \Delta y^{+} \Delta z^{+}\right]$} \\
pulse separation & 200 & 100 & {$[\mu \mathrm{s}]$} \\
dynamic range & 0.3 to 10.8 & 0.6 to 11.8 & {$[\mathrm{pixel}]$} \\
\hline vectors per sample & 13113 & 13113 & \\
number of samples & 2975 & 2100 & \\
\hline
\end{tabular}

TABLE 7.2: Relevant parameters for the characterisation of the experiment performed $18 \mathrm{~m}$ behind the leading edge of the flat plate in the $y z$-plane of the turbulent boundary layer flow.

Two similar experiments have been performed independently. In the first experiment the spatial location of all light-sheet planes was identical and the time separation between a pair of velocity fields being acquired was varied (see left plot of figure 7.2). This allows to study the changes of the velocity structures with time. In the second experiment the time-separation between a pair of measured velocity fields was altered as before but, in addition, the measurement planes were spatially shifted in stream-wise direction by $40 \mathrm{~mm}\left(\Delta x^{+} \approx 300\right)$ as indicated in figure 7.1 and in the right plot of figure 7.2. Thus it was possible to select a flow pattern at an upstream position and to measure the structural changes as a function of the spatial distance between the light-sheet pairs and of the time interval between the two acquisitions 
of the data. As the flow structures and their spatial organisation change while they are transported downstream, the various correlation values yield information about their life history. This is in some extent similar to the image analysis in PIV where the position, size, shape and intensity of the signal-peak in the correlation domain yield statistical information about the size and shape of the particle images themselves as well as information about the magnitude, direction and homogeneity of the displacement of the particle ensemble in between the two illuminations. The main difference lies in the fact that the fluid mechanical correlations presented in the following are ensemble averages calculated over hundreds of realizations, whereas in PIV the ensemble average is replaced by the spatial average of a single realization. This implies that in our case the statistical process leading to the fluid mechanical correlation functions must not necessarily be ergodic as required for PIV image analysis.
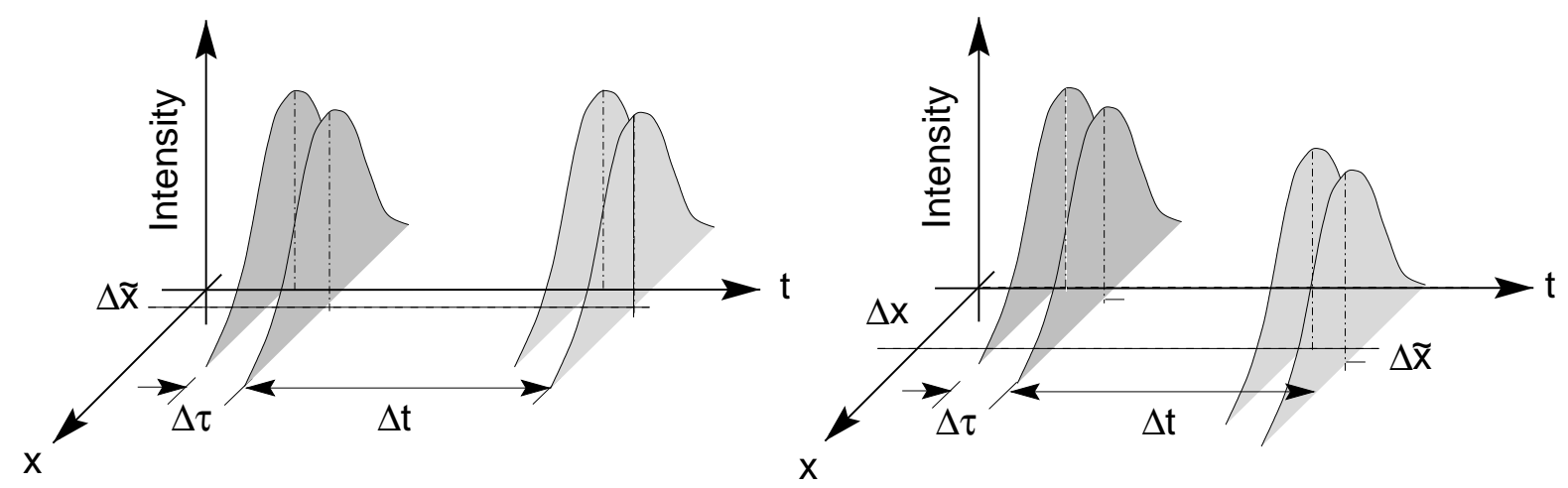

FIGURE 7.2: Optimised light-sheet positioning to reduce loss-of-correlation due to unpaired particle image pairs, induced by out-of-plane motion $(\Delta \widetilde{x}=0.5 \mathrm{~mm})$. Different light-sheet shadings indicate different states of polarisation. Left: Timing diagram for measuring all components of the space-time correlations tensor for $\Delta x=0$ and various $\Delta t$. Right: Timing diagram for measuring all components of the space-time correlations tensor for $\Delta x \neq 0$ and various $\Delta t$.

\subsection{Statistical properties of the log-law region}

To ensure that the experimental arrangement was properly aligned relative to the flow direction, the main statistical flow properties were calculated and compared with the results presented in chapter 5 . The top row of figure 7.3 shows the non-dimensional mean veloc-

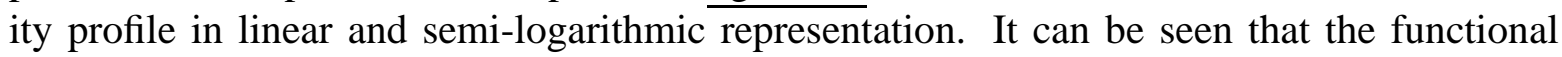
dependence agrees fairly well with the graph from the measurements presented in chapter 5 . This demonstrates the accuracy of the stereoscopic PIV method because the mean streamwise velocity in figure 7.3 corresponds to the out-of-plane velocity component in the present investigation. To estimate the quality of the camera and light-sheet alignment, the statistical properties of the fluctuations in the near-wall region were calculated. The distribution of the velocity fluctuations $u, v$ and $w$, the stream-wise vorticity $\omega_{x}$ and two components of the Reynolds stress tensor ( $v w$ and $u w$ ) are exactly symmetrical so that a high flow quality and very accurate alignment of the laser and recording system can be assumed in the following. Any misalignment of the light-sheet with respect to the wall and mean flow direction or incorrect estimated camera positions would appear as a displacement of the distributions of certain magnitude and direction and, thus, indicate how to improve the setup or to correct the results. 

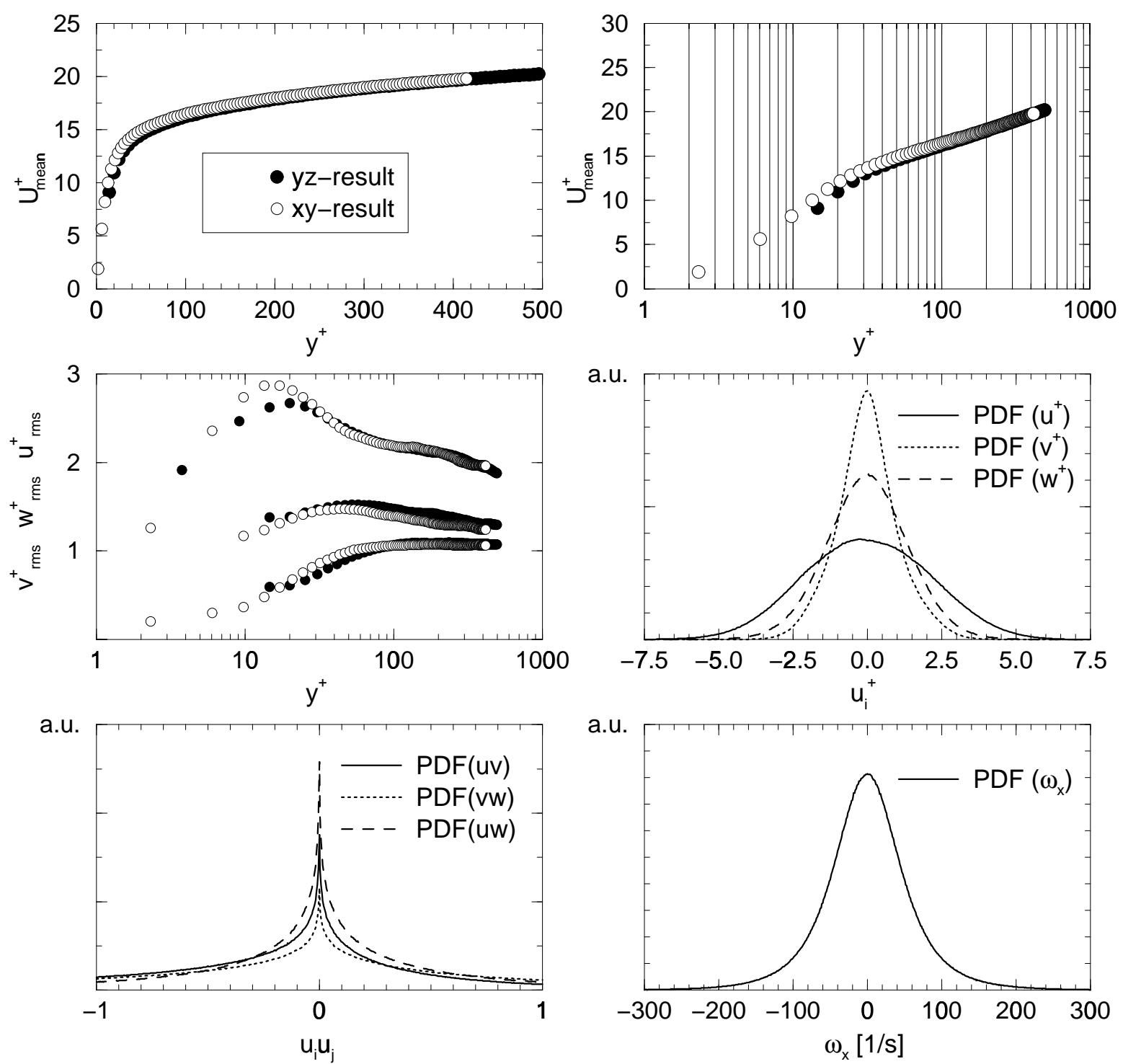

FIGURE 7.3: Flow characteristics. Upper row: Mean velocity profile at $\operatorname{Re}_{\theta}=7800$. Centre row: Intensities and distribution of the velocity fluctuations. Lower row: Distribution of the Reynolds stresses and stream-wise vorticity.

The $u v$ distribution, on the other hand, is not symmetrical and possesses a negative mean value indicating that turbulence is produced on average. Slight differences between both investigations can be only observed in the left graph of the centre row for $y^{+}<30$. In this domain, the maximum of the stream-wise and wall-normal velocity fluctuations is lower with respect to the reference investigation due to the different spatial resolution in wall-normal direction. However, for $y^{+}>30$ the values nicely match apart from the $w$ component of the velocity fluctuation which is slightly underestimated in the $x y$-investigation. This can be explained by the fact that the out-of-plane motion in the $x y$-investigation is quite small referring to the inplane motion in contrast to the $y z$-investigation. This is supported by the hot-wire comparison shown in figure 5.3 on page 77 . It can be seen that the PIV results match with the hot-wire investigations for the $u$ and $v$ component while a slight systematic deviation can be observed in case of the $w$ component. However, it should be mentioned that this systematic difference 

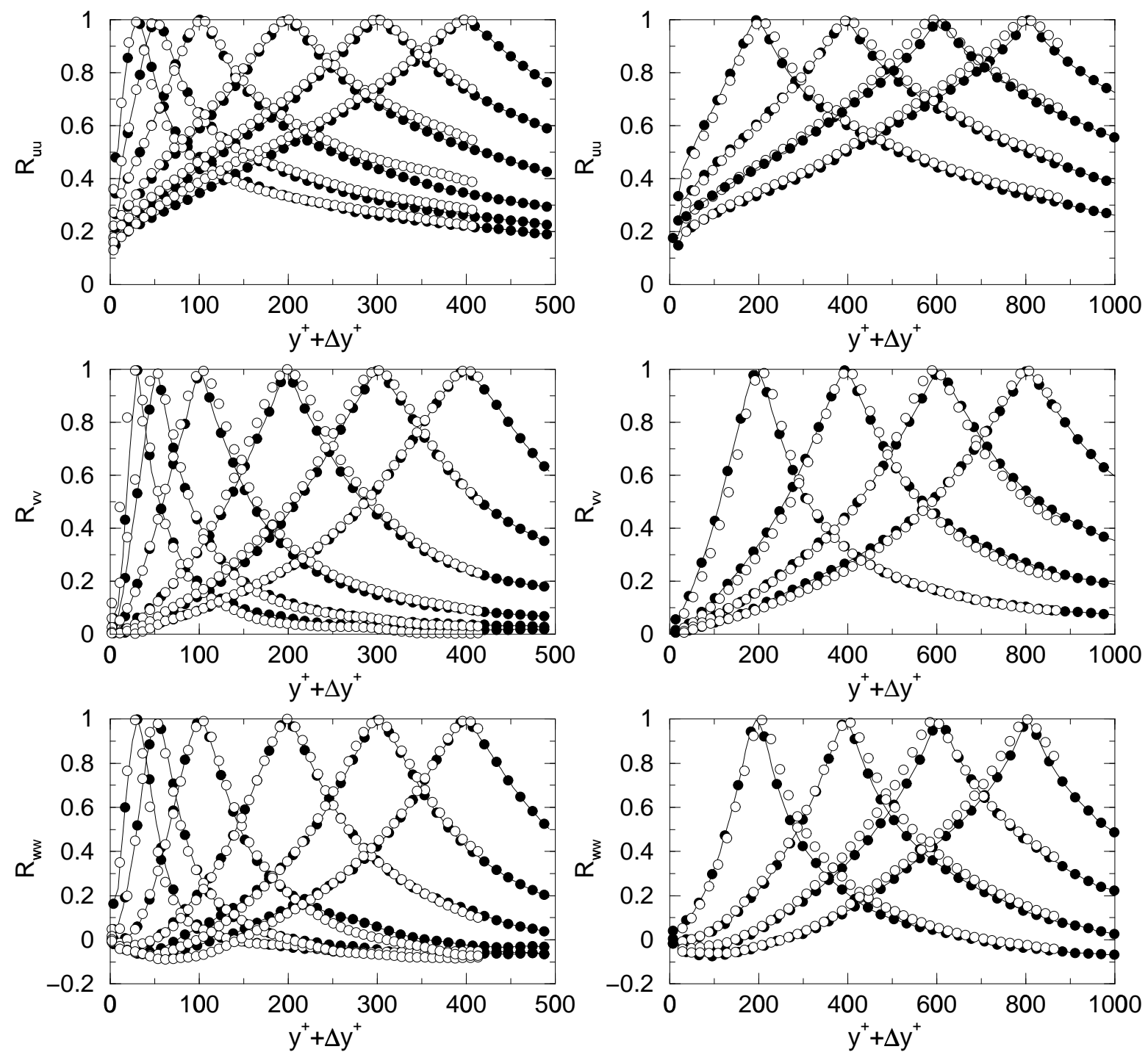

FIGURE 7.4: Primary two-point correlations measured at $R e_{\theta}=7800$ (left) and 15000 (right) and comparison with results from $x y$-investigation (opaque symbols).

does not affect the calculation of the correlations at all. Figure 7.4 shows the dependence of the primary correlations on the location of the fixed point for $R e_{\theta}=7800$ (left column) and $R e_{\theta}=15000$ (right column) in comparison with the results presented in chapter 5 (opaque symbols). Generally it can be seen that the agreement is very good, although the location of the fixed point is not exactly identical due to the different grid applied for the calculation of the velocity vectors. Thus, it can be concluded that the measurement technique is very reliable even if the orientation of the measurement plane is normal with regard to the main flow direction. Based on this result various correlations will be presented in the following in order to complete the results presented in the previous chapters. 


\subsubsection{Spatial correlations with $\Delta x=0$}

The following sequence of figures reveals for $R e_{\theta}=7800$ the three primary correlations of the velocity fluctuations for various wall distances of the fixed point as indicated it the caption or by the location of the maximum. The distance between the contour levels is 0.05 and the solid lines indicate a positive correlation value. Clearly visible is the different size and shape of the correlations and the variation with the wall distance. While the $R_{u u}\left(y^{+}, y^{+}+\right.$ $\Delta y^{+}, \Delta z^{+}$) correlation, shown in figure 7.5, is nearly circular in shape for $y^{+}>100$, the size of the correlation measured at $y^{+}=30$ (upper left plot) decreases quite rapidly in span-wise direction. The extention in wall-normal direction on the other hand measures several hundred wall-units. The short width of the correlation in $z^{+}$-direction implies the relation with the near-
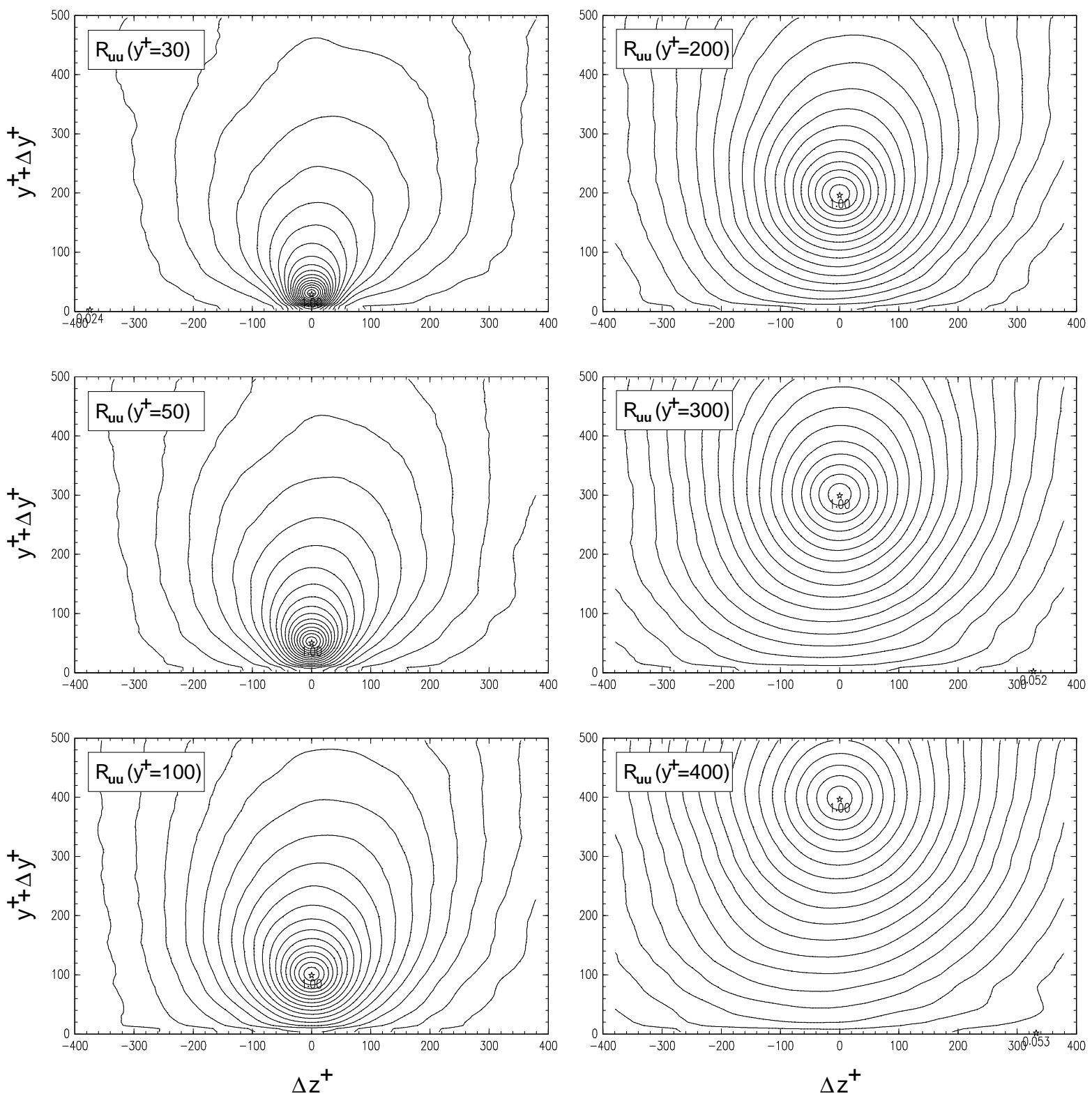

FigURE 7.5: $R_{u u}\left(y^{+}, y^{+}+\Delta y^{+}, \Delta z^{+}\right)$correlation measured at $y^{+}=30,50,100,200,300,400$ (upper left to lower right). 


\section{Investigation of the yz-plane}

wall structures presented in the previous chapter, and the $R_{v v}\left(y^{+}, y^{+}+\Delta y^{+}, \Delta z^{+}\right)$correlation, shown in the upper left image of figure 7.6, indicates the spatial extent of the dominant flow structures which are moving towards and away from the wall. With increasing wall distance from the fixed point, the correlations increase in size but it should be noted that the total size is always below the size of the $R_{u u}$ correlation. When the $R_{w w}\left(y^{+}, y^{+}+\Delta y^{+}, \Delta z^{+}\right)$ correlation in figure 7.7 is considered, it can be concluded that the various primary correlations are always stretched in the direction of the velocity fluctuation. However, the dimension of the stretching which is related to the region where a correlated motion can be observed is quite different. Another interesting feature is the butterfly shape of the $R_{w w}$ correlation, especially for $y^{+}<50$. The fact that the correlation is further away from the wall with increasing $\left|z^{+}\right|$ indicates that the influence of a span-wise motion being present at $y^{+}=30$, for example, is quite limited. This might be related to the fact that the span-wise motion is restricted by the
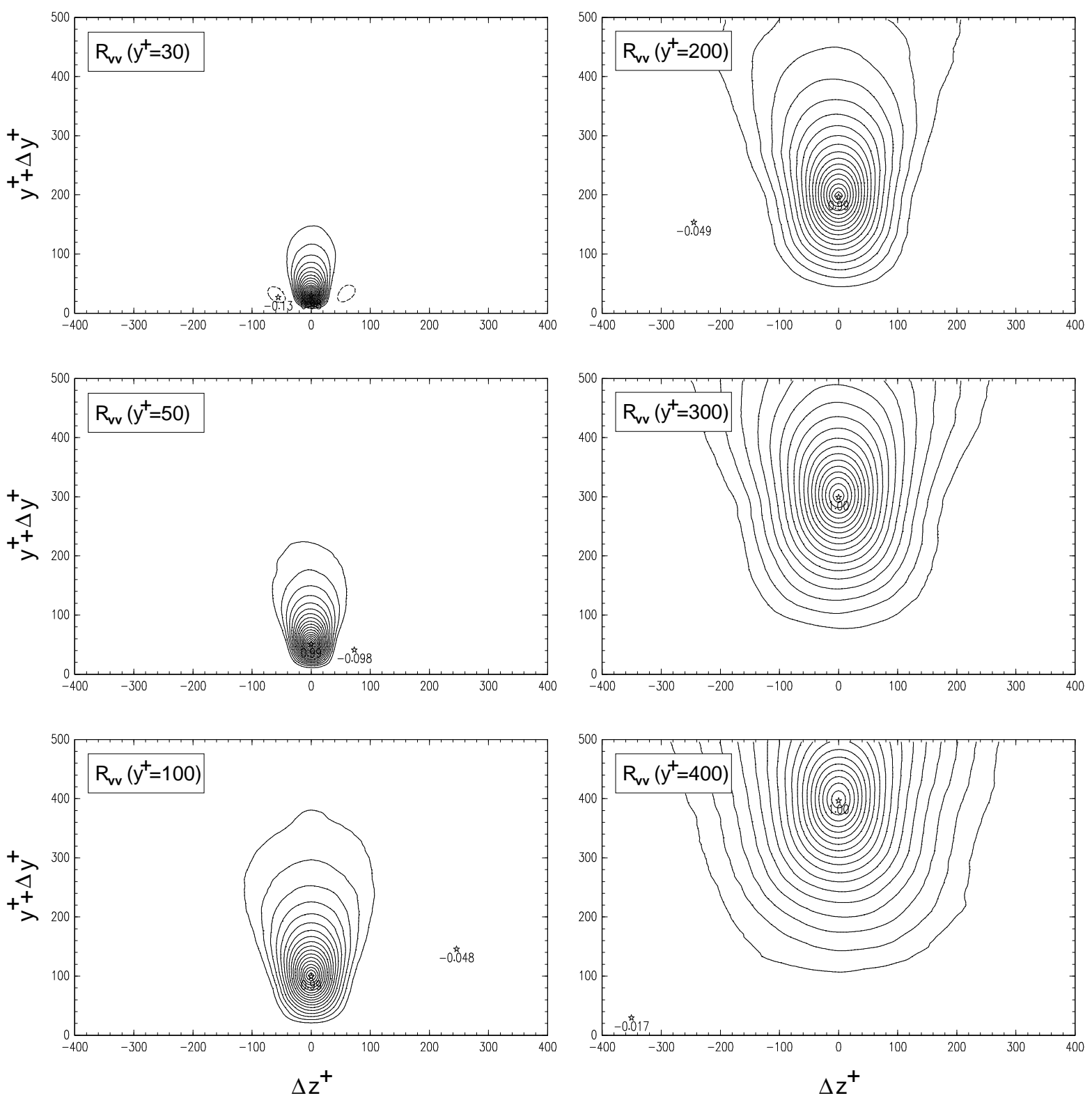

FigURE 7.6: $R_{v v}\left(y^{+}, y^{+}+\Delta y^{+}, \Delta z^{+}\right)$correlation measured at $y^{+}=30,50,100,200,300,400$. 

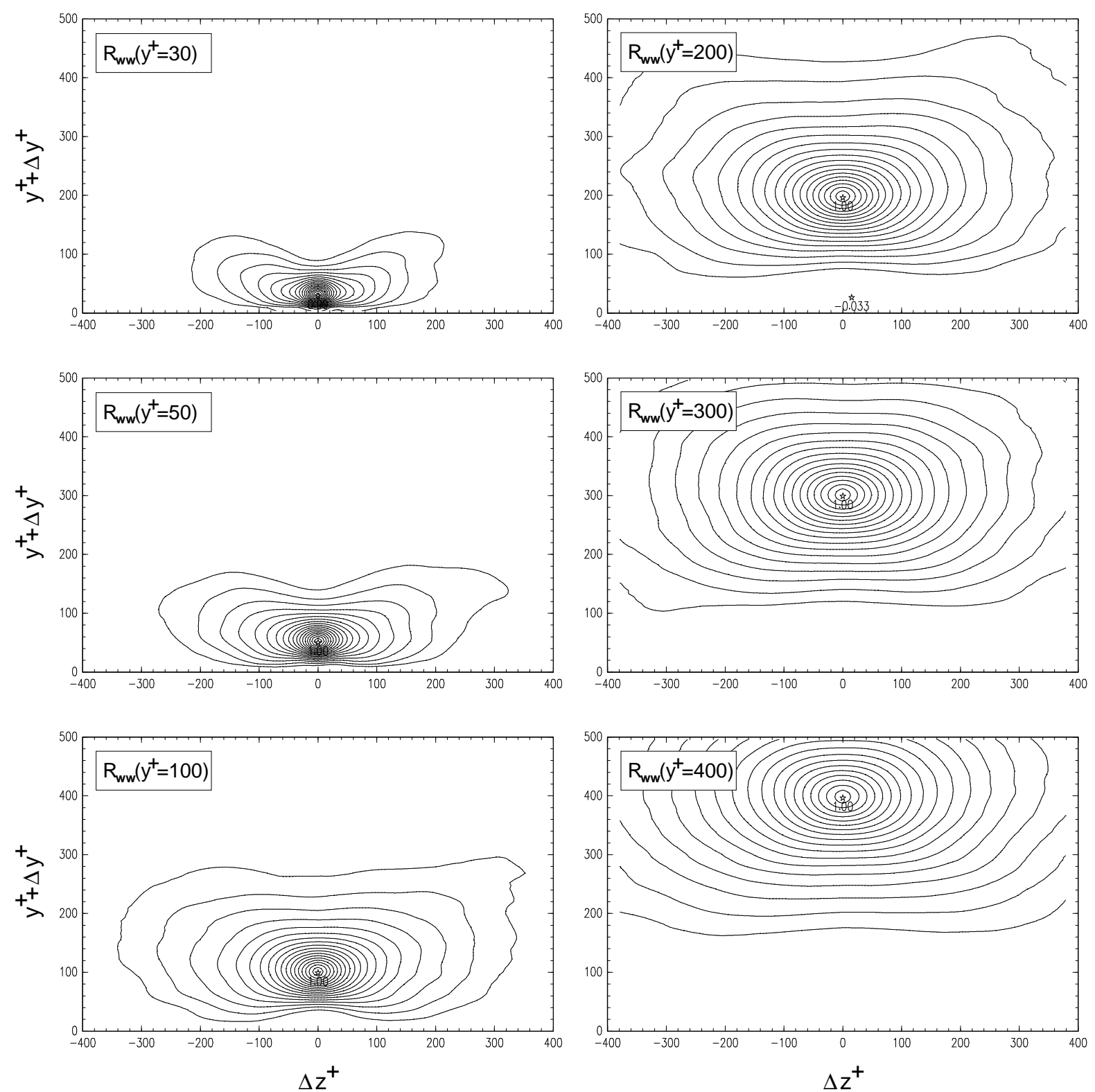

FIGURE 7.7: $R_{w w}\left(y^{+}, y^{+}+\Delta y^{+}, \Delta z^{+}\right)$correlation measured at $y^{+}=30,50,100,200,300,400$.

presence of the streaks which are attached to the wall according to the section 6.4. However, at $y^{+}=100$ the situation is reversed. In this case the correlation function drops down to zero with $y^{+} \rightarrow 0$ at $z^{+}=0$ but for larger $\left|z^{+}\right|$a significant correlation with the near-wall motion can be found.

\subsubsection{Spatial cross-correlations with $\Delta x=0$}

The left column in figure 7.8 shows the $R_{v u}$ cross-correlation function measured at $y^{+}=$ $30,50,100$ (top to bottom) with the $v$ component fixed while the $u$ signal was shifted in the $y$ and $z$ directions. The right column reveals the opposite case where the $u$ component was fixed while $v$ was shifted. The negative sign of the cross-correlations, represented by the dashed lines, indicates again that the transport of relatively low-momentum fluid outward into higher 

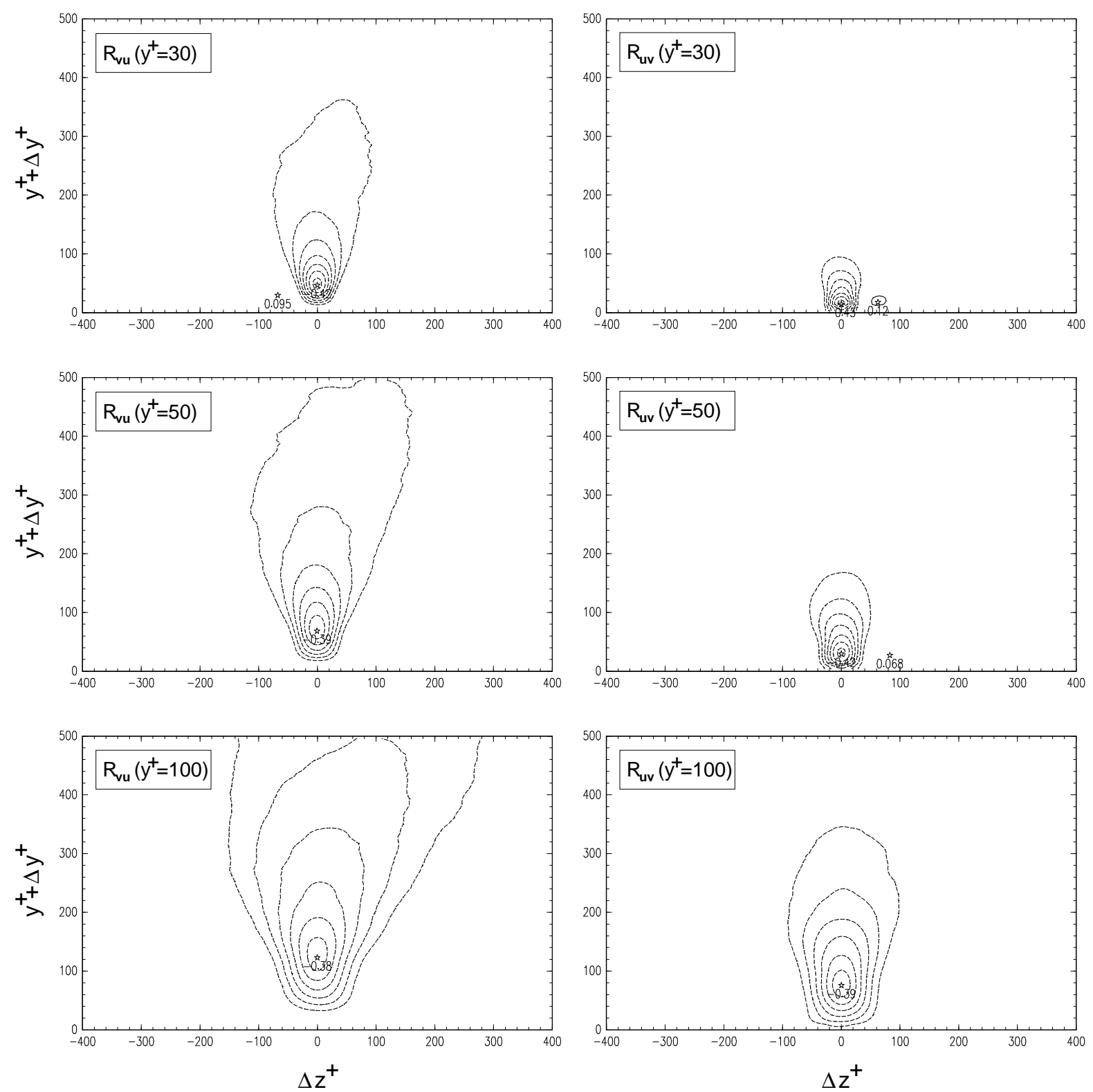

FIGURE 7.8: $R_{v u}\left(y^{+}, y^{+}+\Delta y^{+}, \Delta z^{+}\right)$and $R_{u v}\left(y^{+}, y^{+}+\Delta y^{+}, \Delta z^{+}\right)$correlation measured at $y^{+}=$ $30,50,100$.

speed regions ( $u<0$ and $v>0$ ) and the movement of high-momentum fluid toward the wall into lower speed regions $(u>0$ and $v<0)$ are the predominant processes in the near-wall region. In addition it can be seen from the different size of the correlation functions that in the left case the region, where a correlated motion between both velocity components can be observed, is significantly larger with respect to the case shown in the right column. This implies that the wall-normal motion is strongly affected by the stream-wise fluctuations while the effect of the wall-normal fluctuations on the stream-wise motion is quite limited. This is evident from the equation of motion which states that the energy from the mean motion is transferred into the stream-wise fluctuation at first before a transfer into the $v$ and $w$ component takes place. In addition, it becomes visible from the location of the maximum in figure 7.8 that in the case where the $v$ component is fixed, the maximum of correlation appears at larger wall 

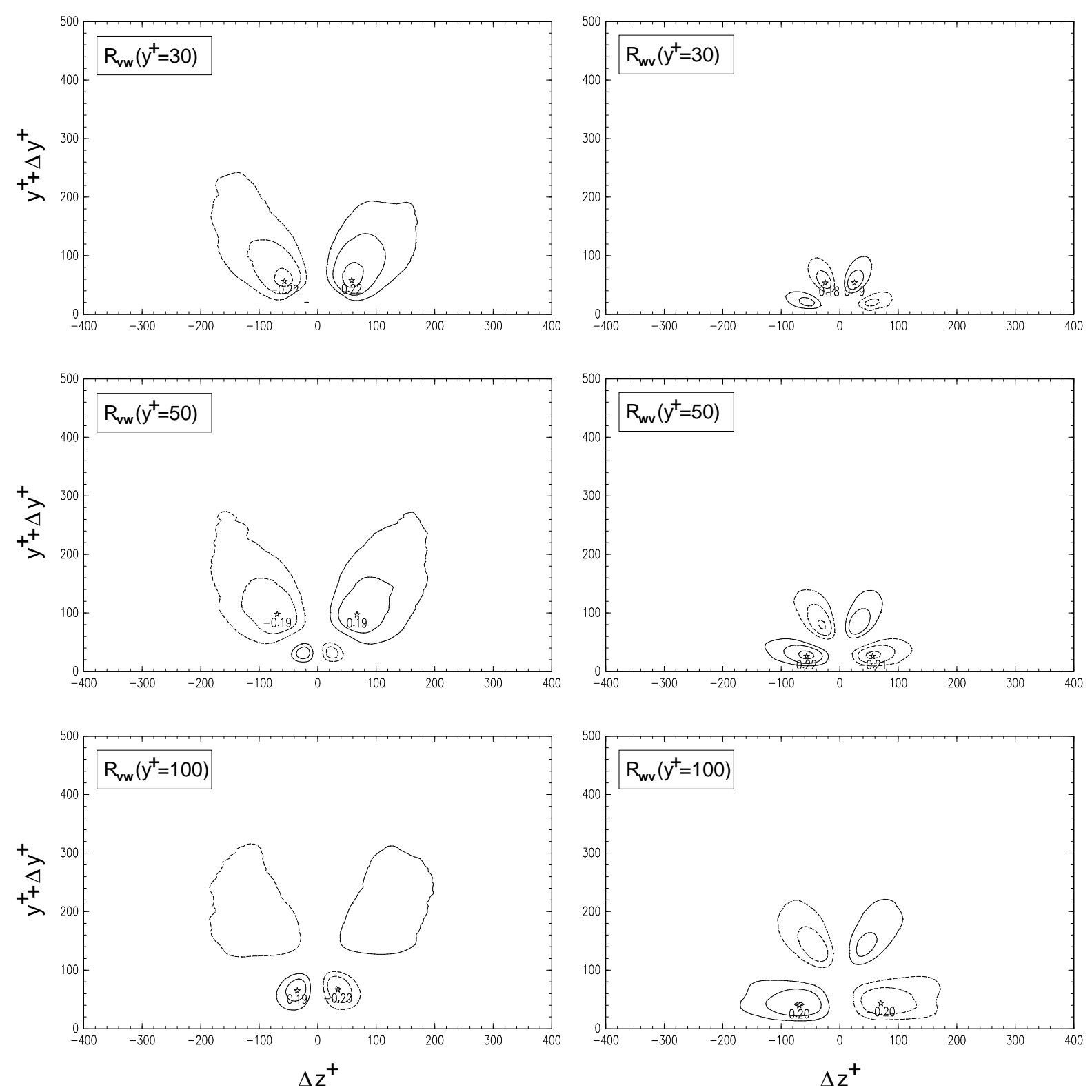

FIGURE 7.9: $R_{v w}\left(y^{+}, y^{+}+\Delta y^{+}, \Delta z^{+}\right)$and $R_{w v}\left(y^{+}, y^{+}+\Delta y^{+}, \Delta z^{+}\right)$correlation measured at $y^{+}=$ $30,50,100$.

distance with respect to the location of the fixed point, while in the case where the $u$ component was fixed the maximum occurs closer to the wall. The same behaviour can be observed when the cross-correlation between the wall-normal and span-wise component of the velocity is considered. The left column in figure 7.9 displays the $R_{v w}$ correlation function measured at $y^{+}=30,50,100$ (top to bottom). Here the $v$ component was fixed while $w$ was shifted in the two homogeneous directions and the right column reveals the opposite case where the $w$ component was fixed while $v$ was shifted. It can be seen that the structures presented in the left column are larger for all wall locations. In addition, a significant change in the organisation of the correlation can be observed when the location of the fixed point is altered. In case of the correlation presented in the right column the structural features remain constant with increasing wall distance and only the size varies. The value of the maximum is comparable for 
all cross-correlations. In order to examine the dynamical properties of the flow represented by the correlations in the left column, a positive span-wise velocity fluctuation which is located at the fixed point will be assumed first. It can be seen from the sign of the correlation that positive $w$ fluctuation induces a vertical motion towards the wall, which is located upstream of the horizontal velocity structure (negative $z$ values), and a motion away from the wall located downstream of the coherent structure. The argumentation can be reversed when a motion with $w<0$ is considered. At larger wall distance it can be deduced from the secondary peaks near the wall that a horizontal motion in positive $z$ direction $(w>0)$ induces also a quite local downstream motion, but the location of this coherent motion is relatively close to the location of the fixed point with respect to the position where an upward motion can be observed. In addition, it can be concluded from the increasing height of the correlation value that the coherence of this near-wall motion increases if the location of the fixed point is farther away from the wall. When the correlations shown in the right column of figure 7.9 are analysed in the same way, it can be seen that a vertical motion at the fixed point location with $v>0$ induces a horizontal motion towards $\Delta z^{+}=0$ in the near-wall region and away from the centre at higher wall locations. A vertical motion towards the wall, on the other hand, induces a horizontal motion away from the centreline at $\Delta z^{+}=0$ due to continuity. As the correlations do not allow to differentiate between primary and secondary motion it is also conceivable to interprete the results in a different way. For example, it can also be stated that a horizontal motion towards the centreline induces a vertical motion away from the wall at the

location of the fixed point. This argumentation was usually applied for the interpretation of the results in chapter 6 . The basis for this interpretation is the assumption that the kinetic energy of the vertical motion is not sufficient to alter the turbulence structure to a large extent, especially near the wall. This can be deduced from the rms-profiles shown in figure 7.3. Anyway, the three dimensional size, shape and interaction of the flow structures is now fully determined if the results presented in chapter 5 and 6 are taken into account.

\subsection{Spatio-temporal correlations with $\Delta x=0$}

To examine the dynamical aspects of the organised flow structures, the temporal evolution of the primary correlations as a function of the wall-normal coordinate $y^{+}$and time delay $\Delta t^{+}=t u_{\tau}^{2} / \nu$ will be analysed in the following. It might be argued that it is easier to perform such an analysis when the orientation of the measurement plane is parallel to the main flow direction. However, it should be kept in mind that due to the small size of the correlations in span-wise direction the results may be strongly biased if the measurement plane is not perfectly aligned with respect to maximum of the correlation in physical space, or, if the 3D correlation is slightly tilted in span-wise direction due to the limited number of samples, for example. Both effects can be avoided by using the experimental configuration applied here. To obtain correct results a two-dimensional correlation was calculated from which two orthogonal lines being parallel to the coordinate axis were extracted, and the location of the global maximum was used to determine the intersection point of the lines to be extracted (the one-dimensional correlation function depends on the coordinates of the points from which the values are taken from the four-dimensional correlation function). For the calculation of the two-dimensional correlation functions a line at a particular wall location was extracted from the measurement acquired at time $t$ and correlated with the result measured at $t+\Delta t$. The distance between both measurement planes is zero in order to study the convective de- 
cay of the flow structures. Figure 7.10 and figure 7.11 reveal the primary correlations measured at $\operatorname{Re}_{\theta}=7800$ and figure 7.12 and figure 7.13 show the corresponding results for the $R e_{\theta}=15000$ investigation. Clearly visible is the different size and shape of the correlations and the variations in the temporal decay. Due to the different size of the correlation functions
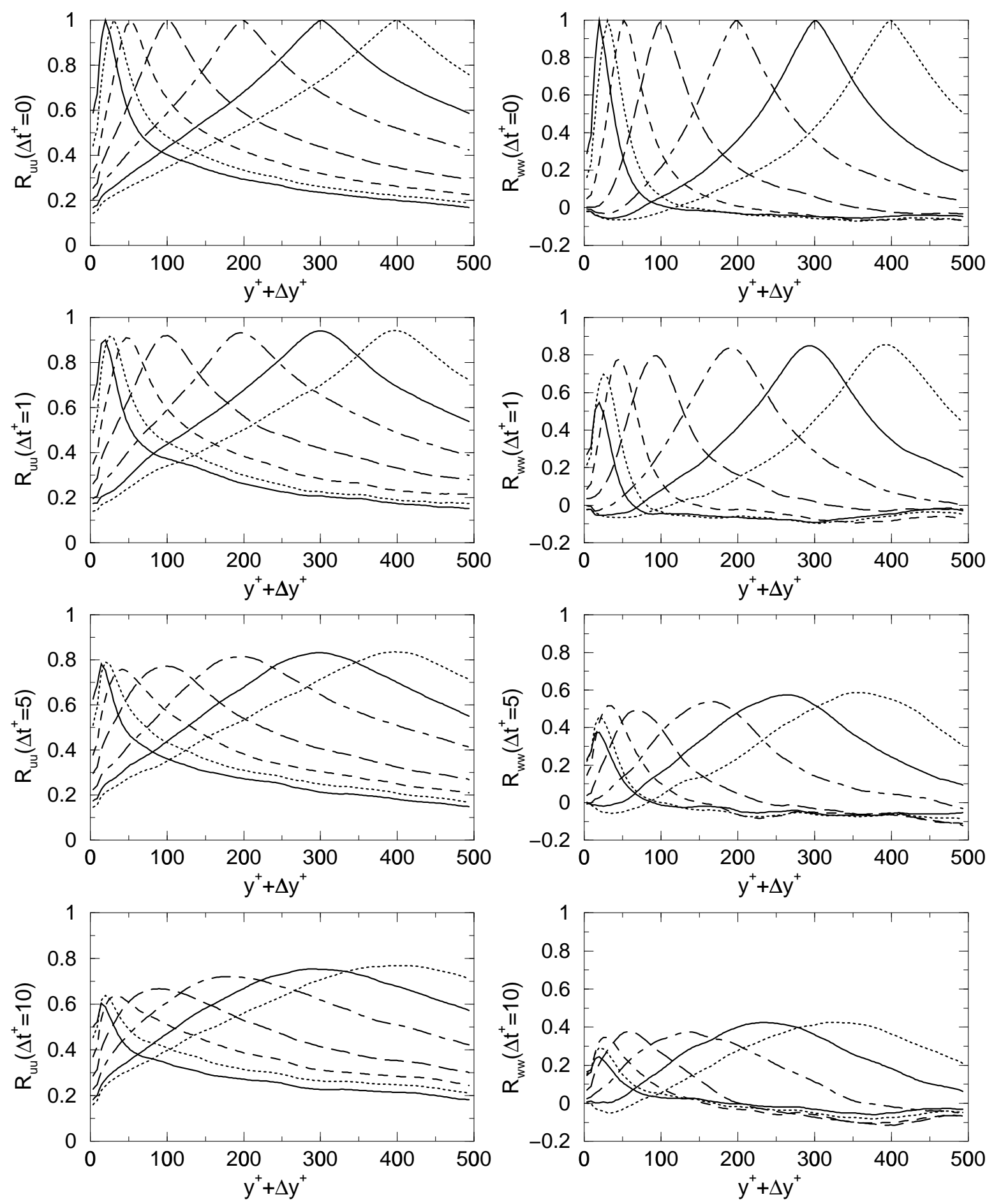

FIGURE 7.10: $R_{u u}$ (left) and $R_{w w}$ (right) correlation functions measured at $R e_{\theta}=7800$ and $y^{+}=$ $20,30,50,100,200,300,400$ (see location of the maximum). 
in stream-wise direction according to section 5.3.2 it is evident that the maximum of $R_{v v}$ decreases quite rapidly with increasing temporal delay and vanishes already for $\Delta t^{+}=10$ at $R e_{\theta}=7800$ while the other correlations still show a significant correlation which is above 0.6 for $R_{u u}$ and around 0.4 in case of $R_{w w}$. Another interesting effect becomes visible when the correlation functions in the near-wall region are considered. It can be seen that the $R_{v v}$ and $R_{w w}$ correlations decrease quite rapidly with respect to $R_{u u}$ and relative to the decrease of the correlation function at larger wall locations of the fixed point. This implies that structural changes within the near-wall region are quite large. When the width of the correlation and the curvature around the maximum are considered, the total size decreases only very little with increasing temporal separation but the curvature of the maximum becomes weaker. This indicates the dissipation of the small-scales due to action of the viscosity. The large-scales, on the other hand, are only weakly affected within the same time. This implies that the structures which become visible for large time separations can be considered as a low-pass representation of the dominant velocity structures. This point will be discussed in the next section.
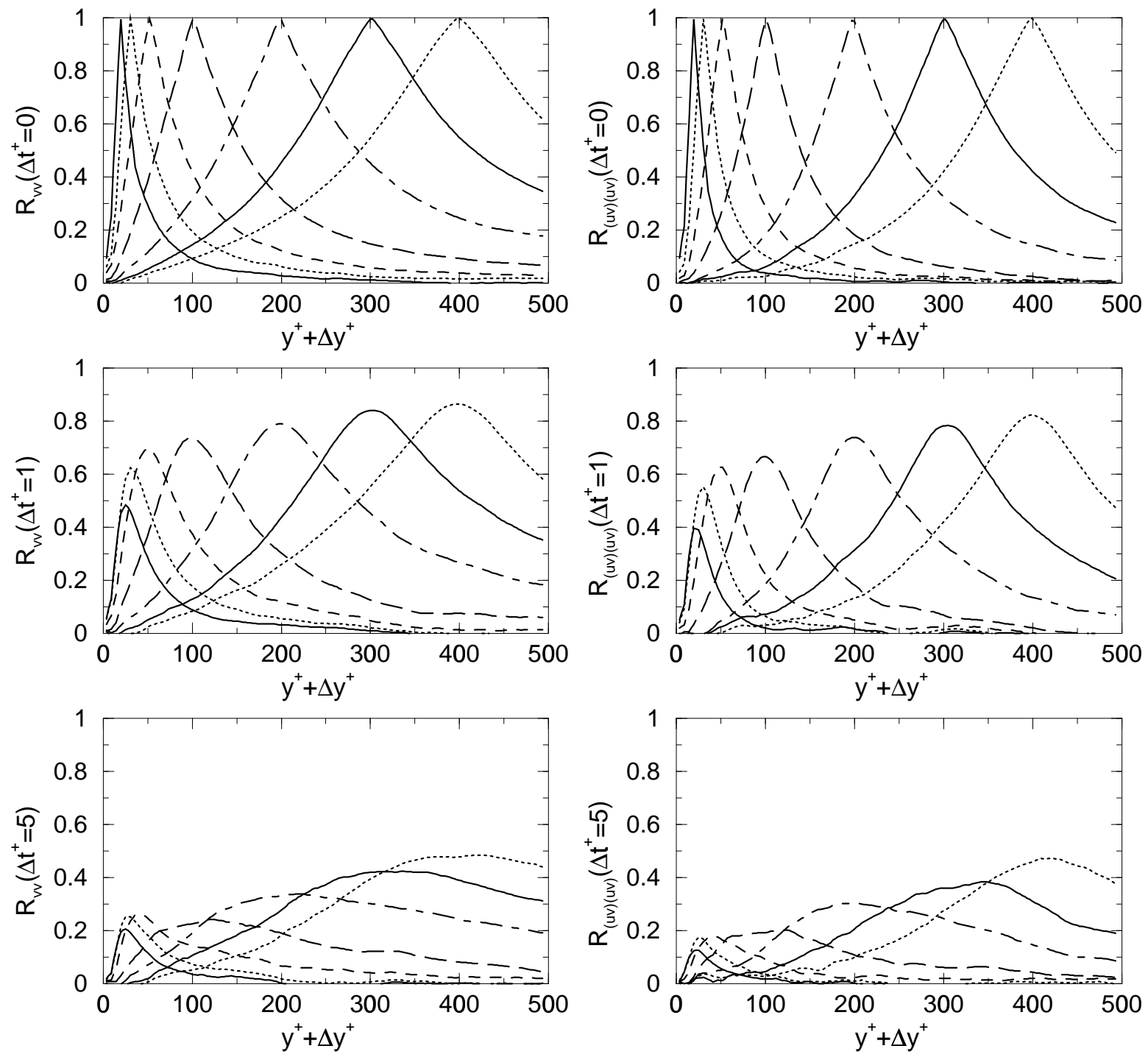

FIGURE 7.11: $R_{v v}\left(\Delta t^{+}\right)$(left) and $R_{(u v)(u v)}\left(\Delta t^{+}\right)$(right) correlation functions measured at $R e_{\theta}=$ 7800 and $y^{+}=20,30,50,100,200,300,400$ (see location of the maximum). 


\subsection{Spatio-temporal correlations with $\Delta x=0$}

To estimate the average decay of the flow structures being responsible for the production of turbulence, the $R_{(u v)(u v)}\left(\Delta y^{+}\right)$correlation was calculated, see right column of figure 7.11 and 7.13. The rapid decay of $R_{(u v)(u v)}$ for increasing $\Delta t^{+}$is mainly caused by the small correlation
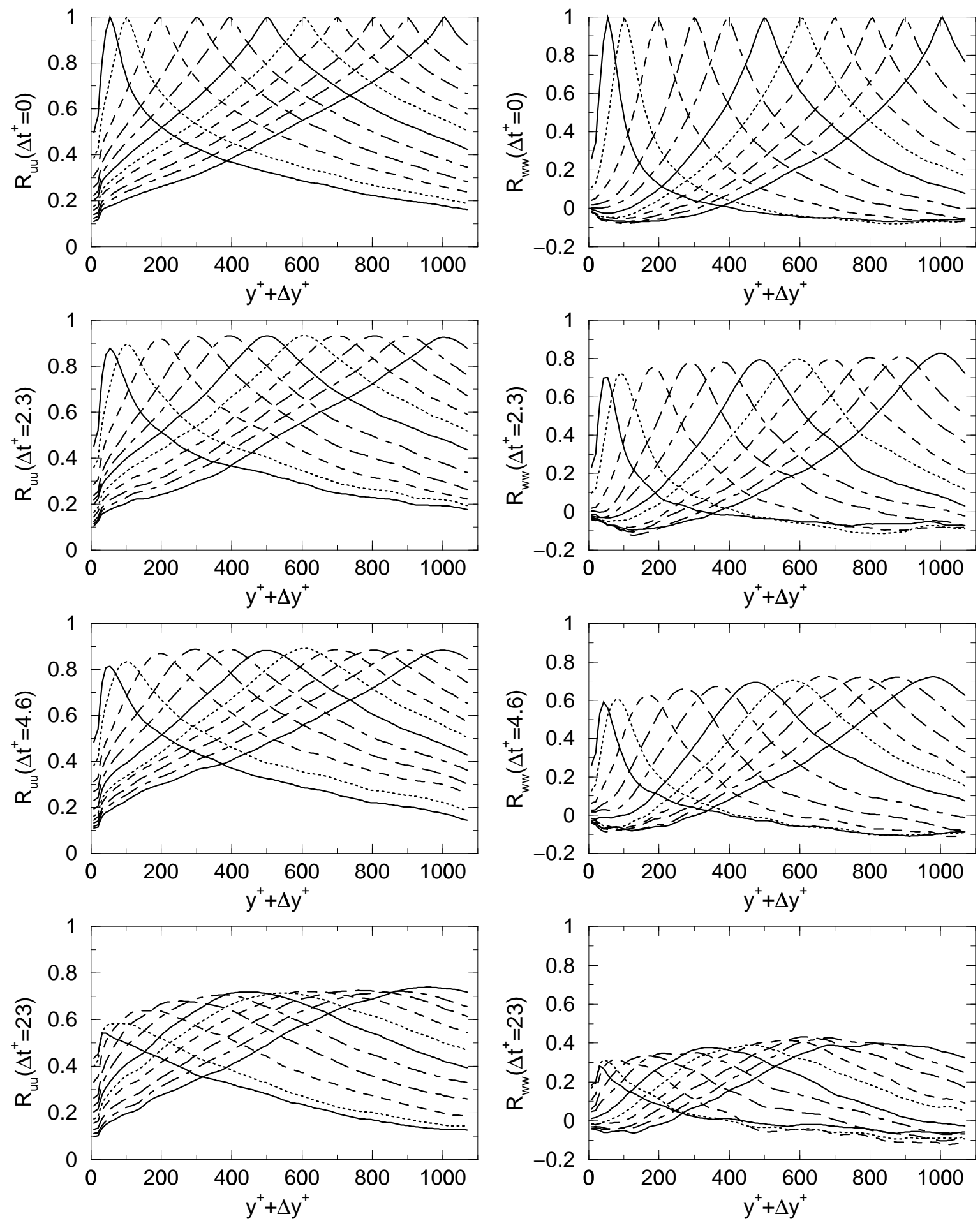

FIGURE 7.12: $R_{u u}\left(\Delta t^{+}\right.$) (left) and $R_{w w}\left(\Delta t^{+}\right.$) (right) correlation functions measured at $\operatorname{Re}_{\theta}=15000$ and $y^{+}=50,100,200,300,400,500,600,700,800,900,1000$ (see location of the maximum). 
length of $R_{v v}$. Another important fact is the shift of the $R_{u u}\left(\Delta y^{+}\right)$and $R_{w w}\left(\Delta y^{+}\right)$maximum with increasing $\Delta t^{+}$. This is shown more clearly in figure 7.14 for various wall distances of the fixed point. The symbols indicate the position of the maximum and the legend reveals the corresponding position in wall-units. The graphs labelled by the solid symbols indicate a correlation where a line at a particular $y$-location of the first image measured at time $t$ is correlated with the second image acquired at $t+\Delta t$. The opaque symbols indicate a correlation where a line extracted from the second image at $t+\Delta t$ is correlated with the first image measured at $t$. The asymmetry of the correlation shift for $\pm \Delta t$ indicates again that the flow structures, represented by this particular correlation function, reveal two dominant inclination angles relative to the wall, and it can be seen that the inclination of structures at closer wall distances relative to the location of the fixed point reveal the smaller inclination angle. The analysis of the spatio-temporal correlation functions of the velocity fluctuations and the Reynolds stresses indicate that the statistical properties of the log-law region is quite complex with respect to the
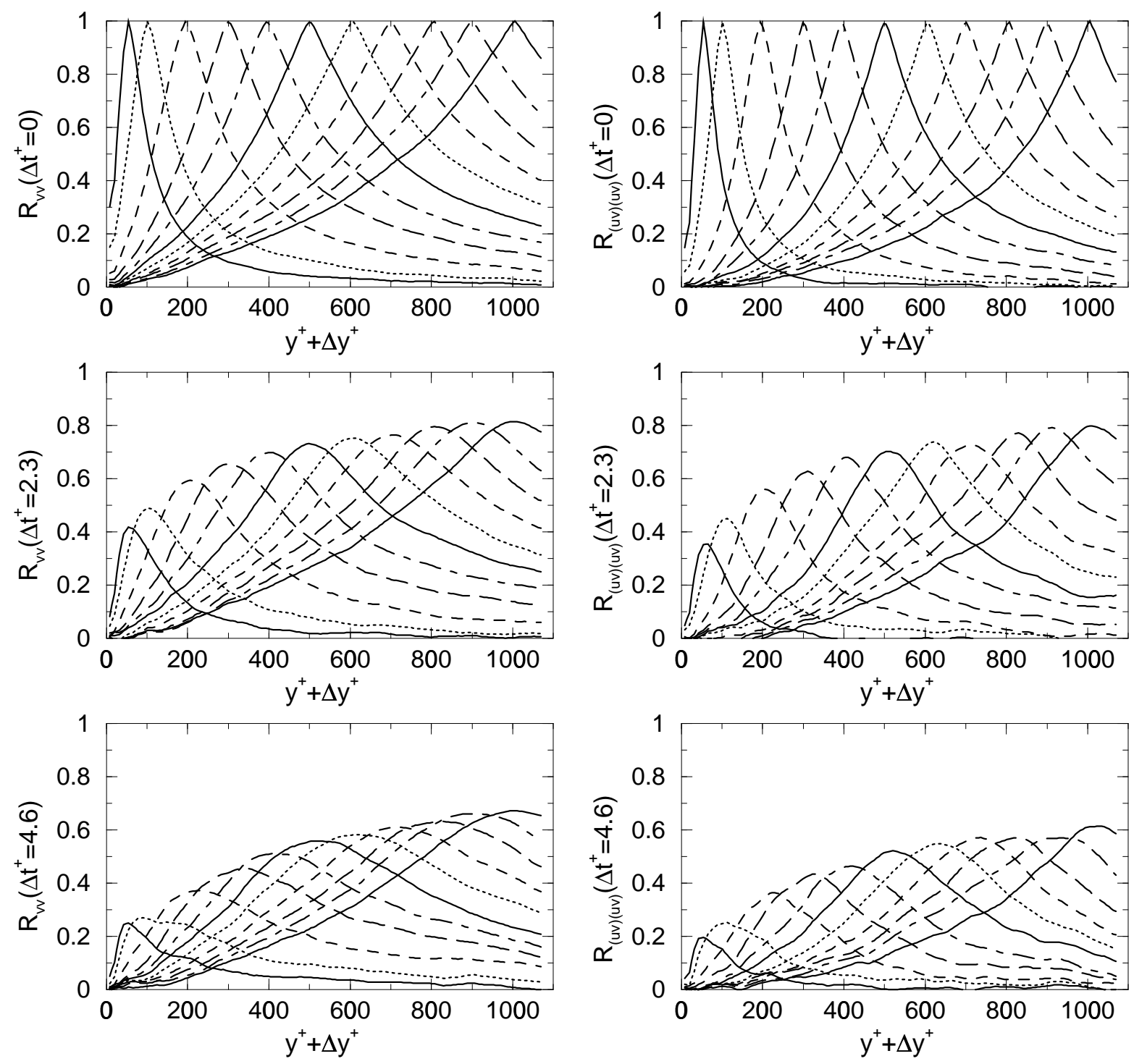

FIGURE 7.13: $R_{v v}$ and $R_{(u v)(u v)}$ correlation functions measured at $R e_{\theta}=15000$ and $y^{+}=$ $50,100,200,300,400,500,600,700,800,900,1000$ (see location of the maximum). 
first order moments of the velocity fluctuations within this domain. It could be shown that the structures of the spatio-temporal auto-correlation functions of the stream-wise $\left(R_{u u}\right)$ and span-wise $\left(R_{w w}\right)$ velocity fluctuations are quite similar (shape, size and temporal decay) when the span-wise and wall-normal dependence is considered, whereas their cross-correlation $R_{u w}$ is necessarily zero due to symmetry. Also, the characteristic shift of the largest correlation value towards higher velocity regions with time agrees well. The wall-normal correlations, on the other hand, are relatively small and their maximum decreases fast with increasing time. As a consequence, the spatial extent of regions where most of the Reynolds stresses are produced must be relatively limited. The correlation function of the stream-wise vorticity component also reveals a short spatio-temporal correlation length and a displacement towards higher velocity regions. Although a consistent interpretation of the presented results is conceivable, the instantaneous velocity fields need to be analysed for further evidence.
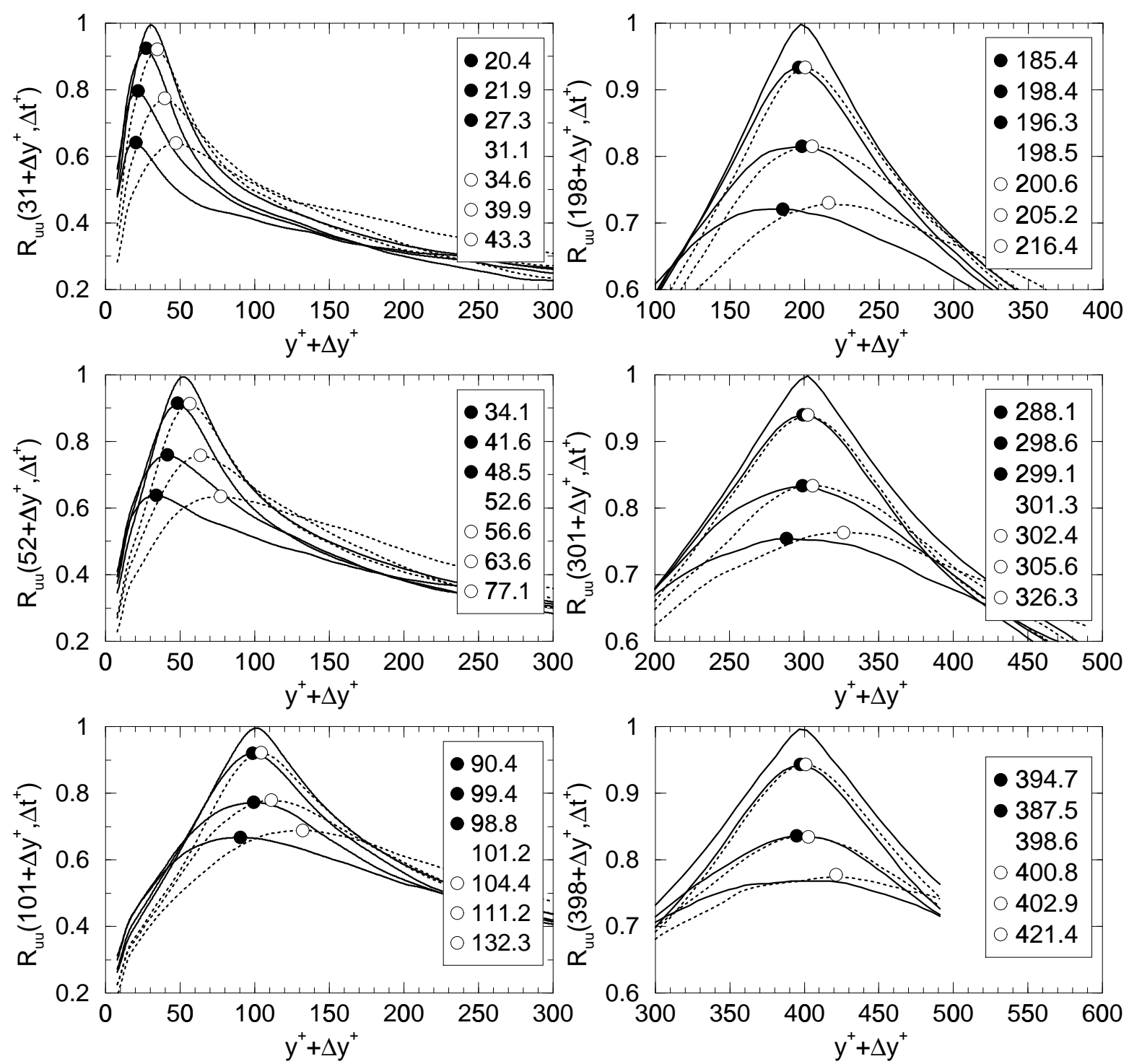

FIGURE 7.14: Representation of the $R_{u u}$ correlation at $R e_{\theta}=7800$ in the vicinity of the maximum for various wall distances $y^{+}$of the fixed point and temporal separation between the measurements $\left(\Delta t^{+}=-10,-5,-1,0,+1,+5,+10\right)$. 


\subsection{Spatio-temporal correlations with $\Delta x \neq 0$}

In this section various one-dimensional spatio-temporal correlation functions calculated from measurements in spatially separated planes will be examined. This particular experimental arrangement allows to select a flow pattern at an upstream position and to measure the structural changes due to the mean velocity gradients and to the turbulent motion as a function of the spatial distance between the light-sheet pairs and the time delay between the measurements. Figure 7.15 to figure 7.17 reveal the primary correlation functions for various wall distances from the fixed point and time delays between the measurements. The results were obtained by cross-correlating the measurements acquired at different locations (325 wall-units streamwise separation between both measurement planes) while the downstream measurement was performed after the upstream measurement as indicated by the axis label. As the local mean velocity is approximately $16 u^{+}$according to figure 7.3 , the structures selected at the upstream
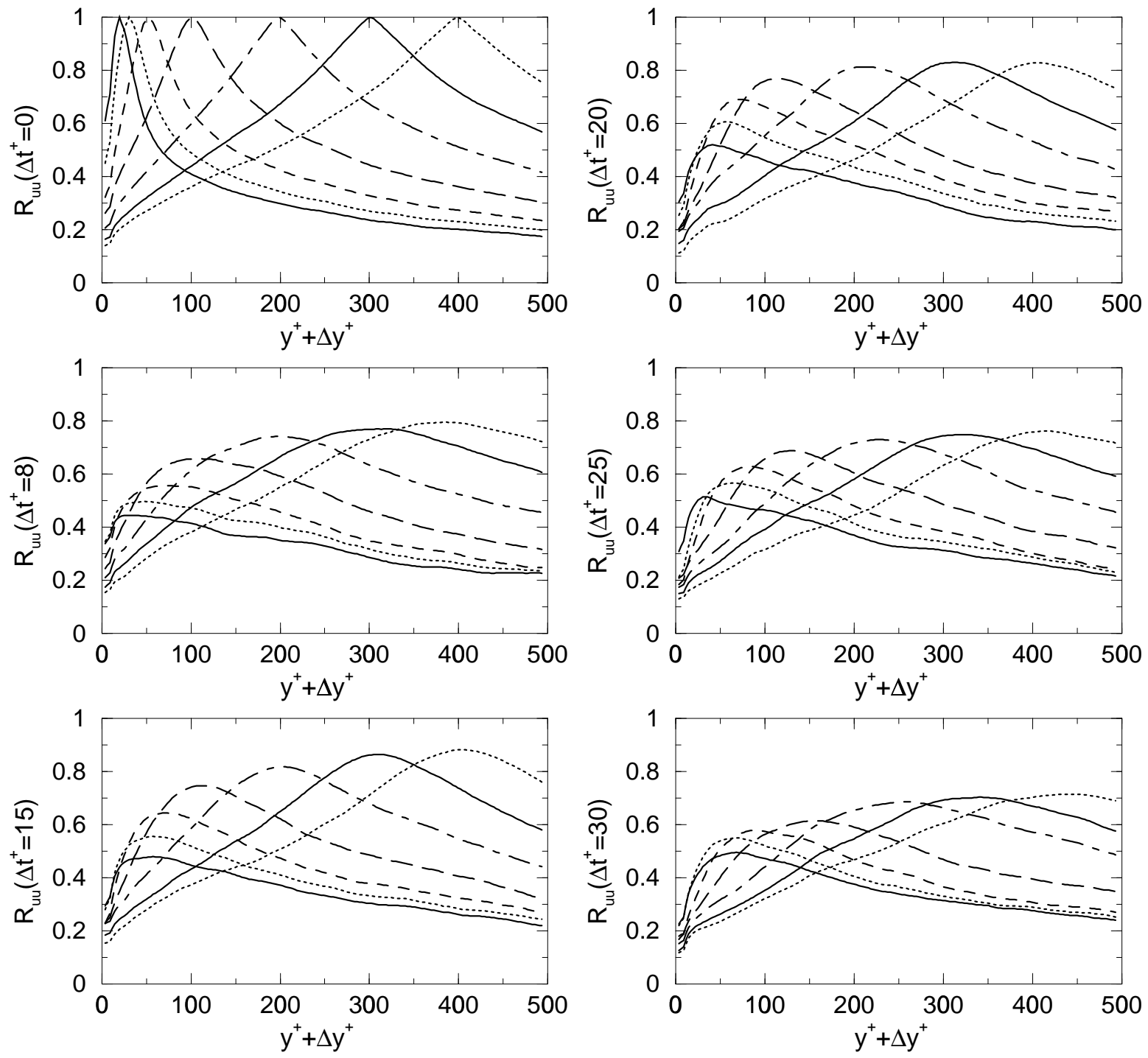

FIGURE 7.15: $R_{u u}$ correlation measured at $R e_{\theta}=7800$ and $y^{+}=20,30,50,100,200,300,400$ (see location of the maximum) for various temporal delays (see axis label). 
location are convected by about 130 wall-units (40\% of the distance between the measurement planes) for a temporal delay of $\Delta t^{+}=8$. Thus, the maximum correlation value is relatively low, especially near the wall, but it slightly increases with increasing $y$ due to the larger correlation length and larger local convection velocity. An other interesting effect is the shift of the maximum towards larger wall distances. However, both observations can be explained by means of the result shown in figure 5.5 on page 80 . When the correlation in the lower left column is considered with the maximum at $y^{+}=50$, the profile at $\Delta x^{+}=130-325=-195$ corresponds to the dashed graph in the left centre row of figure 7.15 , provided the structural changes are negligible, so that it can be assumed that the flow structure convects down-stream with an average velocity of $15 u^{+}$(local mean velocity at $y^{+}=50$ ) over 130 wall-units in stream-wise direction. In this case it can be estimated that the highest correlation value at $\Delta x^{+}=-195$ is 0.54 in the lower left column of figure 5.5, which nicely matches with the results from the present investigation, and the corresponding position of the maximum is at $y^{+} \approx 62$. This implies that the general properties of the correlations can be well understood
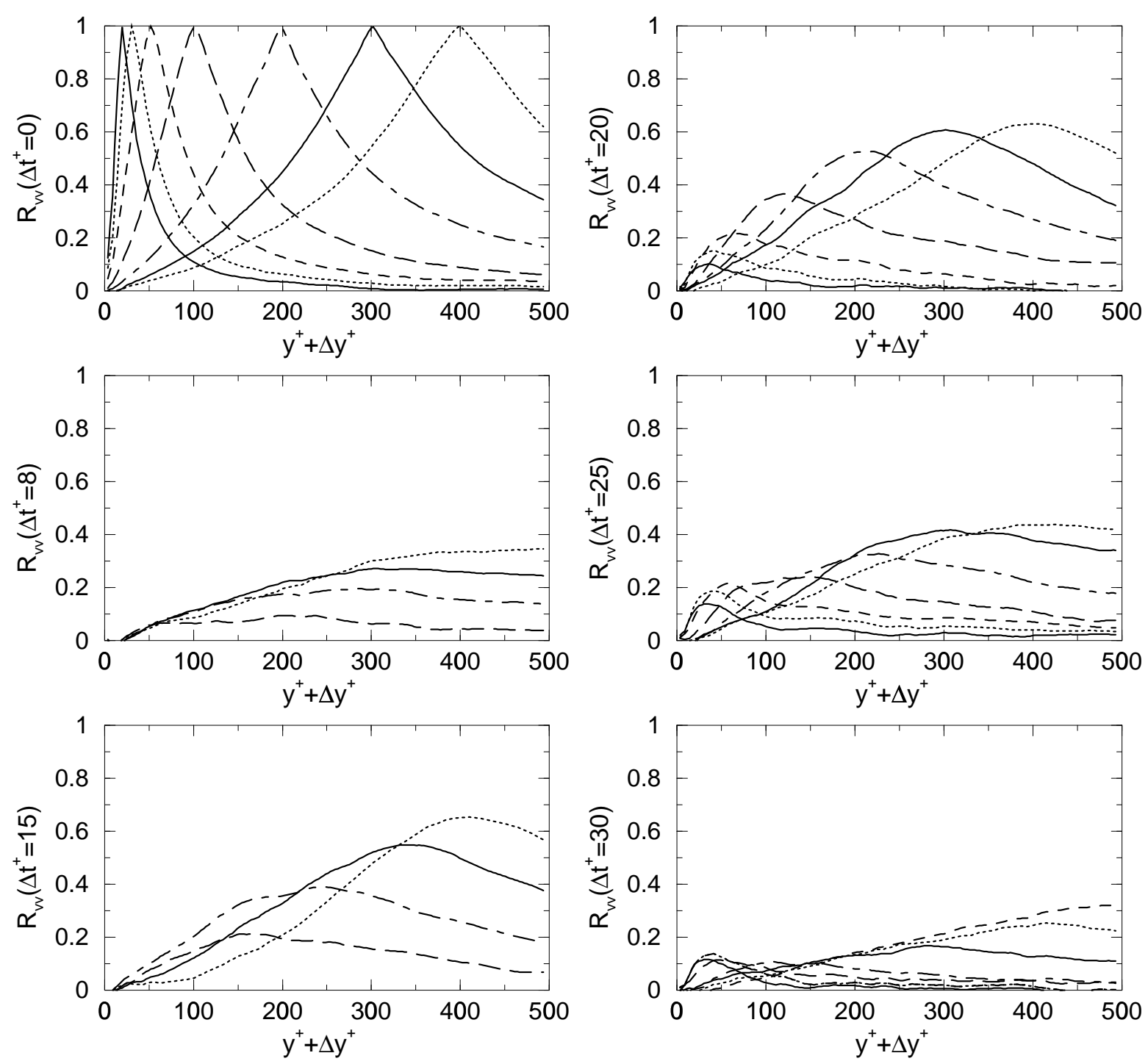

FIGURE 7.16: $R_{v v}$ correlation measured at $R e_{\theta}=7800$ and $y^{+}=20,30,50,100,200,300,400$ (see location of the maximum) for various temporal delays (see axis label). 
by using the results from chapter 5. For $\Delta t^{+}=15$ (lower left row of figure 7.15) the travelling distance is around 240 wall-units ( $75 \%$ of the separation between measurement planes) for the near-wall structures, but the structures at $y^{+}>200$ already reach the second observation plane as the mean convection velocity varies between 18 for $y^{+}=200$ and $19.8 u^{+}$ for $y^{+}=400$. This can be directly estimated from the height of the correlation maximum in the upper right graph of figure 7.15. While the correlation maximum already decreases for fixed point locations at $y^{+}>200$ a gradual increase can be observed for the near-wall correlations. All correlation maxima increase with increasing $\Delta t$ while the maximum correlation always remains lower near the wall. As the difference in height is quite large, structural changes or variation in the organisation of the structures closer to the wall (strong variation of the structure location from one time-step to the next) need to be assumed for this effect. This assumption is strongly supported by the fact that both, integral and dissipative, length scales significantly increase in the two last rows. Translated in the language of PIV image analysis, this effect corresponds to loss of pairs (resulting in a lower correlation coefficient)
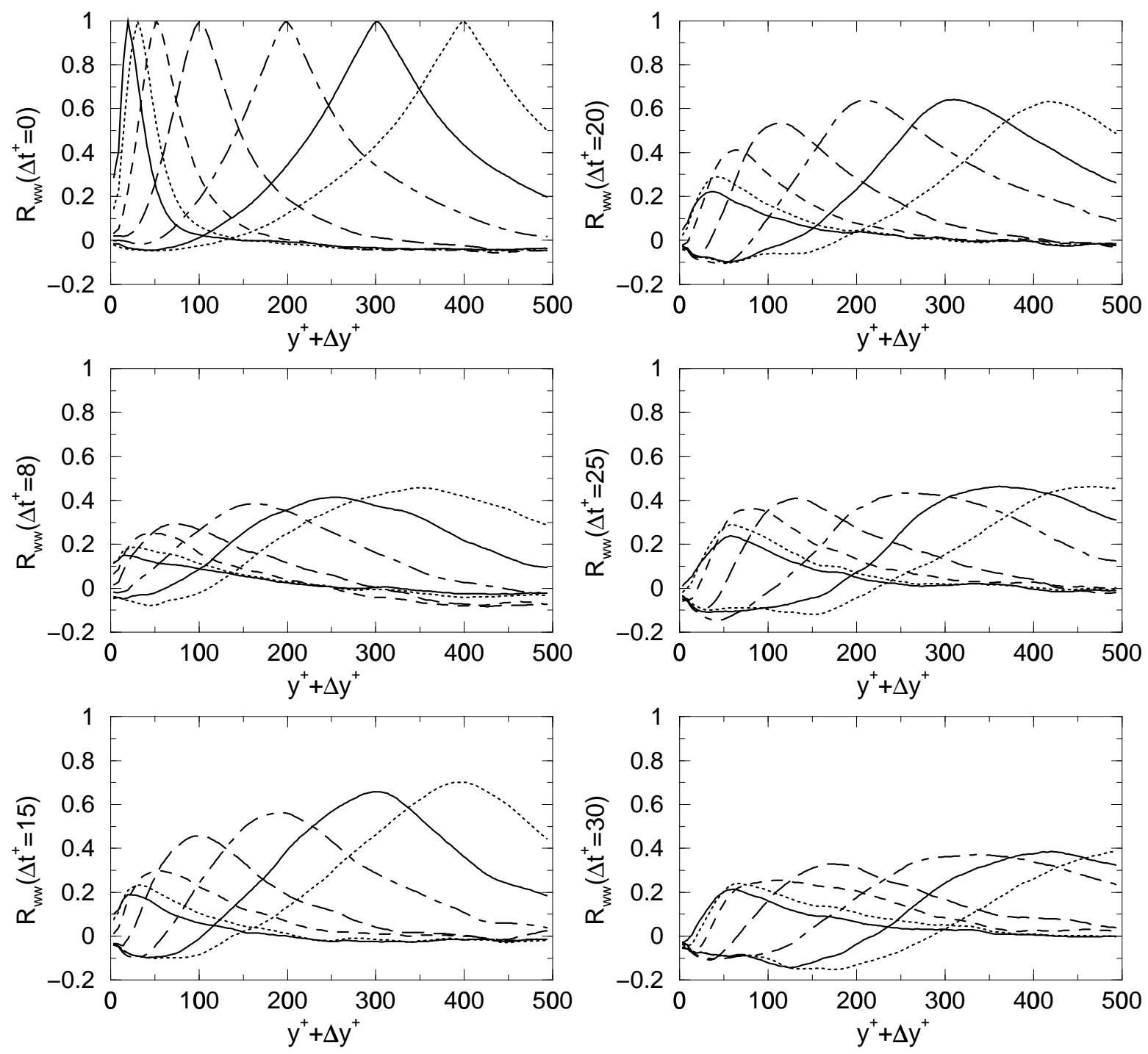

FIGURE 7.17: $R_{w w}$ correlation measured at $R e_{\theta}=7800$ and $y^{+}=20,30,50,100,200,300,400$ (see location of the maximum) for various temporal delays (see axis label). 
accompanied by structural differences in the successively recorded particle image patterns due to inhomogeneous displacement which leads to a broadened shape of the correlation peak.

In case of the $R_{v v}$ correlation shown in figure 7.16 no peaks can be observed with $\Delta t^{+}=8$ for the near-wall correlations due to the small size. However, if the graphs for $\Delta t^{+}=15$ to 30 are considered, the movement of the $R_{v v}$ correlation through the second measurement plane can be nicely seen. The $R_{w w}$ correlation, on the other hand, shown in figure 7.17, reveals peaks for all wall locations and temporal separations but the height of the correlation is always lower with respect to figure 7.15. This implies that the span-wise motion is less stable according to the structures represented by the $R_{u u}$ correlation. Another interesting feature visible in figure 7.17 is the strong increase of the $y^{+}$location of the maximum as a function of the time separation. This is fully consistent with figure 5.7 on page 82 . Only the shift towards smaller wall locations at $\Delta t^{+}=8$ is surprising. However, this is a necessary consequence of the order of correlation. In the present case a line at $y^{+}$was extracted from the results in the down stream plane and shifted along the field measured upstream. This implies that the the maximum appears at smaller $y^{+}$locations until the maximum of the correlation in figure 5.7 passes the down stream measurement plane. The same holds for the other correlations.

\subsection{Properties of coherent velocity structures}

\subsubsection{Loop-shaped structures}

In this section the significance of the stream-wise vortices for the turbulent mixing in wallbounded flows will be examined and the existence of the loop-shaped structures, highlighted on page 6 will be validated. Loop-shaped structures can be best detected in the $y z$-plane of a turbulent boundary layer (wall-normal span-wise) because these structures are inclined in stream-wise direction [13, 28, 49]. Their footprint is a counter-rotating vortex pair with a strong velocity component being normal to the wall between the vortex pair as indicated in figure 1.3. In planes which are parallel to the wall (stream-wise span-wise) these loops appear as counter-rotating vortices with a typical out-of-plane motion between the vortex cores as illustrated in section 6.4. Thus the basic turbulent mixing process associated with this hairpin vortex is the transfer of low-speed fluid from the wall and high speed fluid towards the wall such that $-\rho \overline{u v}$ becomes positive on average. Since the early flow visualisation experiments performed in a laminar boundary layer, it is generally accepted that looped shaped structures result from a progressive deformation of a span-wise vortex with an initial three dimensional disturbance, as shown in the lower figure on page 6 . The inclination of the vortices on the other hand is explained by means of self-induction of the developing vortex loop, and the stretching is assumed to be a result of the strong velocity gradients present in boundary layer flows [31].

However, it is still a point of discussion if these loop-shape structures are the predominant coherent velocity regions in turbulent boundary layers which are mainly responsible for the turbulent mixing. In the past, many attempts have been made in order to validate the existence and significance of these structures. The continuing discussion implies that no convincing experimental or theoretical evidence could be presented. As the signature of these coherent structures is a counter-rotating vortex pair with a strong velocity component normal to the wall between the vortex pair, as indicated in figure 1.3, they can be easily identified when the measurement plane is perpendicular to the wall and mean flow direction. Figure 7.18 shows two independent velocity fields where many well developed vortex pairs can be observed in 
the near-wall region below $y^{+}<100$ (see circles). Moreover it can be seen that these vortices induce a wall-normal velocity as indicated in figure 1.3. This is fully consistent with the results discussed in section 7.2.2. In addition the red contours, which represent a negative outof plane velocity fluctuation $(u<0)$, show that the stream-wise velocity of the fluid between the vortex pairs is relatively low with respect to the mean motion. This implies that turbulence is produced by these structures as expected and discussed in the previous chapters. The blue contours indicate that the structures are frequently flanked by high-speed fluid.
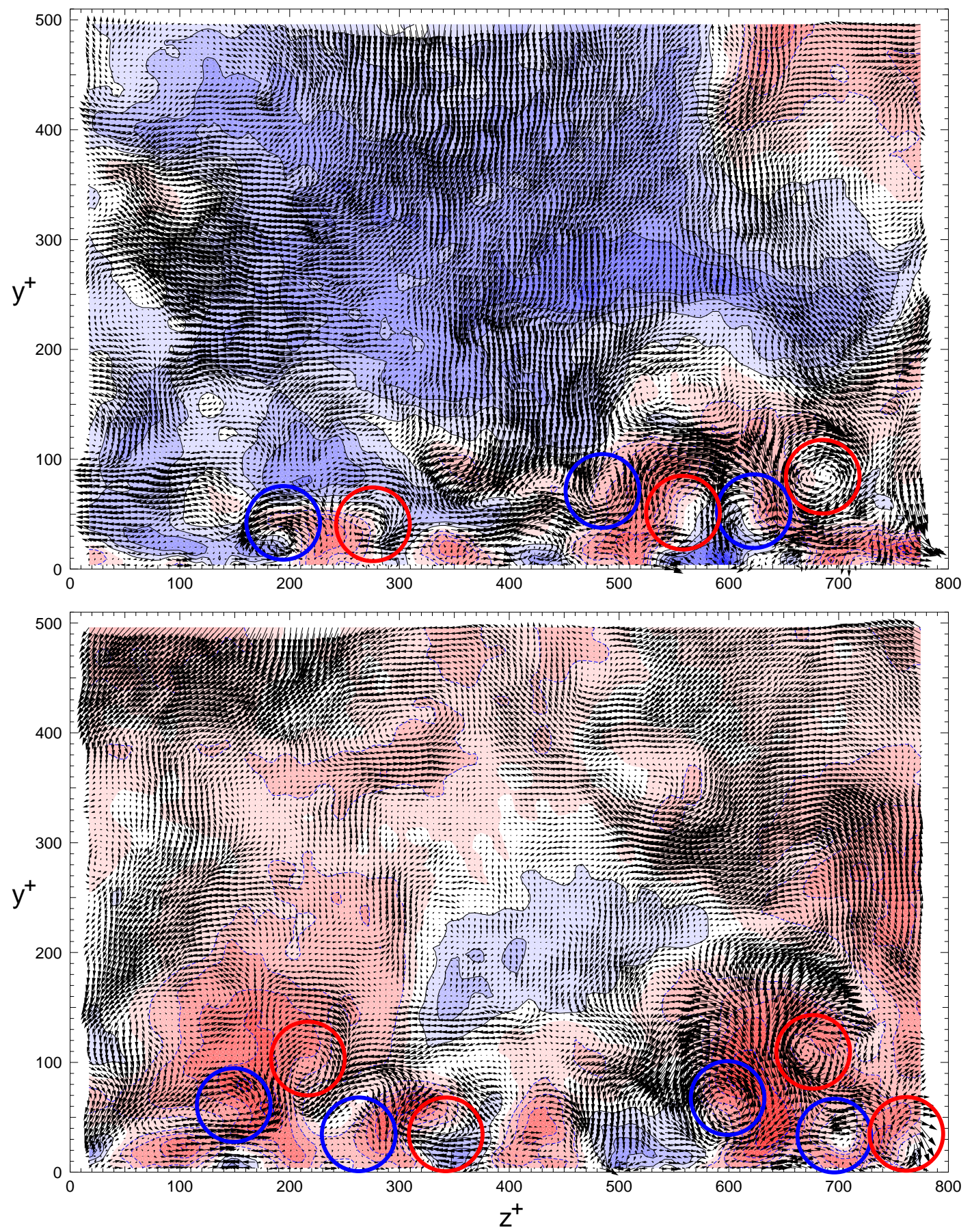

FIGURE 7.18: Velocity fluctuations (blue: $u>0$; red: $u<0$ ) with loop shaped structures, see circles. 


\subsubsection{Sweeps}

In order to interprete the results from the previous chapter it was assumed that the lifting of low-speed fluid from the wall is mainly caused by the high-momentum flow structures which move towards the wall. This interaction is shown exemplary in figure 7.19. Clearly visible is the strong wall-ward motion of a large-scale high-momentum flow structure in the lower image and the generation of looped shaped structures similar to those shown in the previous figure. The upper figure reveals similar structures but it should be noted that the
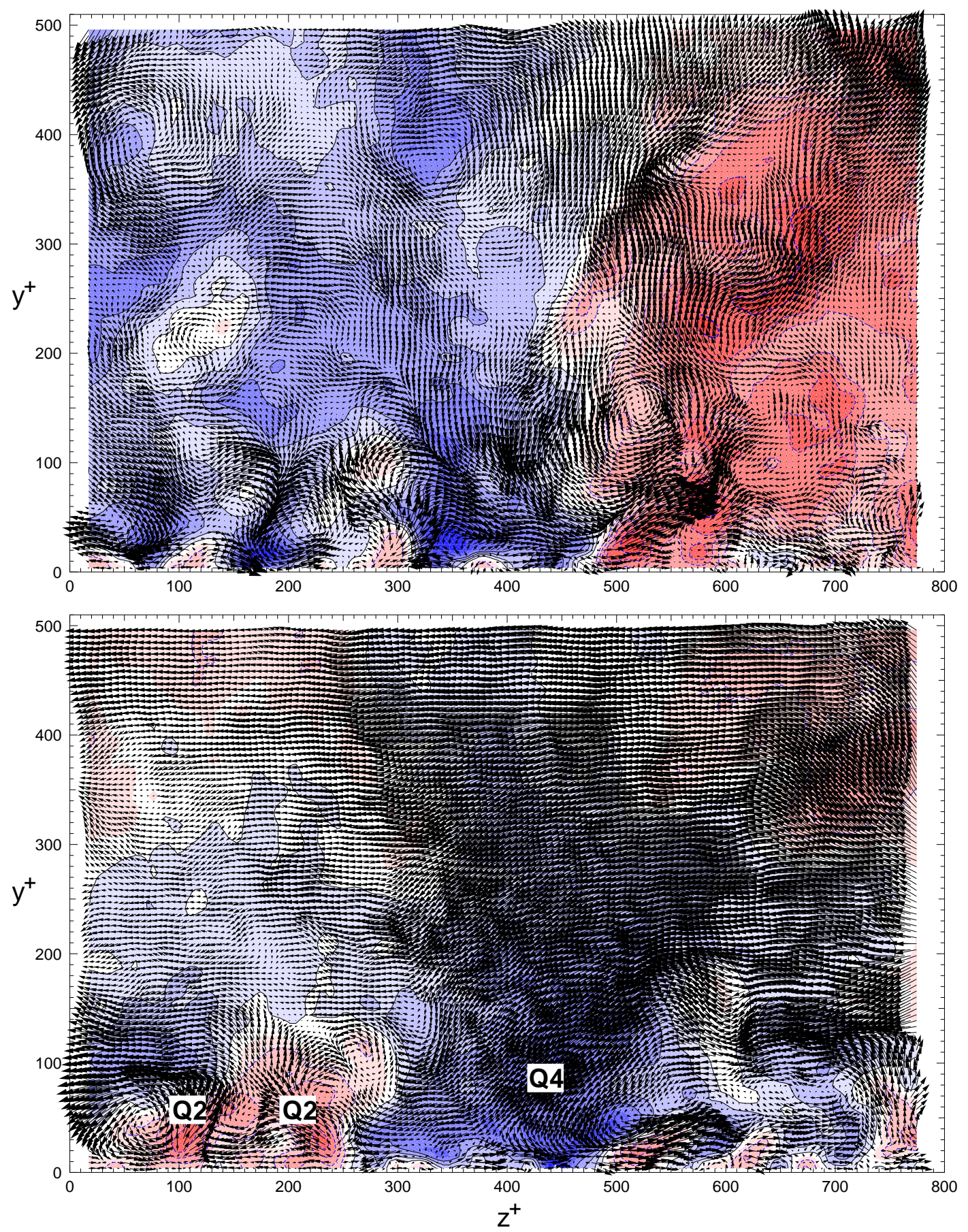

FIGURE 7.19: Same as figure 7.18 but with strong sweeps $(u>0$ and $v<0)$. 
span-wise size of the high-speed flow structures, which reach the wall, is quite small with respect to the structure visible in the lower image. To examine structures which contribute to the turbulent mixing to a large extent, the Reynolds shear stress component was calculated for each sample field and afterwards analysed if the intensity of this quantity was below a given threshold of -0.3 . It was already mentioned that the dominant structures with regard to the magnitude of the Reynolds shear stress component appear quite seldom and do not contribute to the total production of turbulence to a large extent. However, as the structural features are
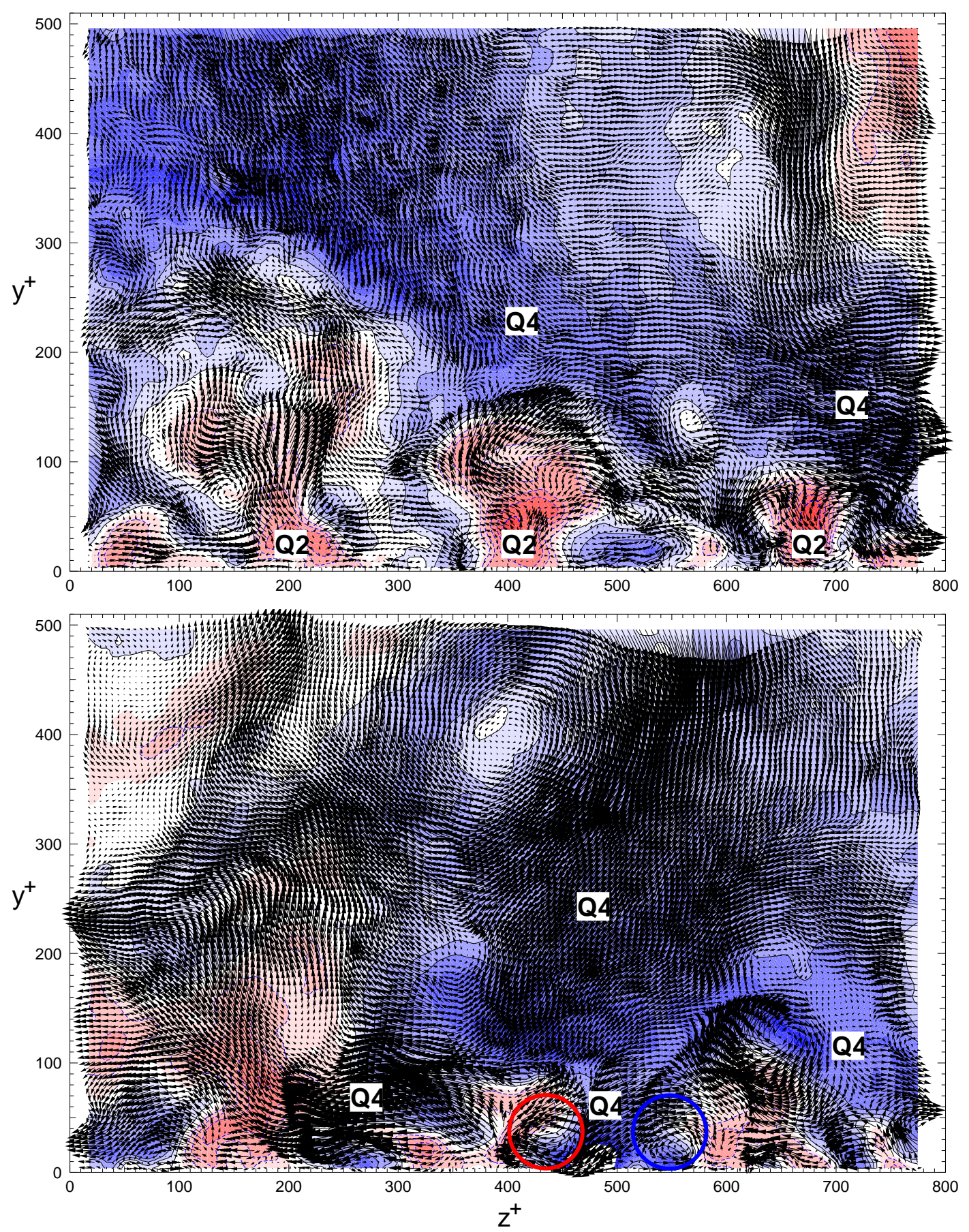

FIGURE 7.20: Same as figure 7.18 but with strong production of turbulence $(u v<0)$. 
assumed to be universal and thus independent on the magnitude of $-\rho \overline{u v}$, it is evident that their geometrical and dynamical properties can be best examined when the effect of the background turbulence, which often deteriorates the analysis of the less intense structures, becomes small in relation to the structures under investigation. Figure 7.20 shows two samples measured independently at $R e_{\theta}=7800$. Clearly visible in both velocity fields is the high-momentum large-scale motion (blue), which moves towards the wall, and the effect when these structures interact in the near-wall region with the low-momentum structures (red).
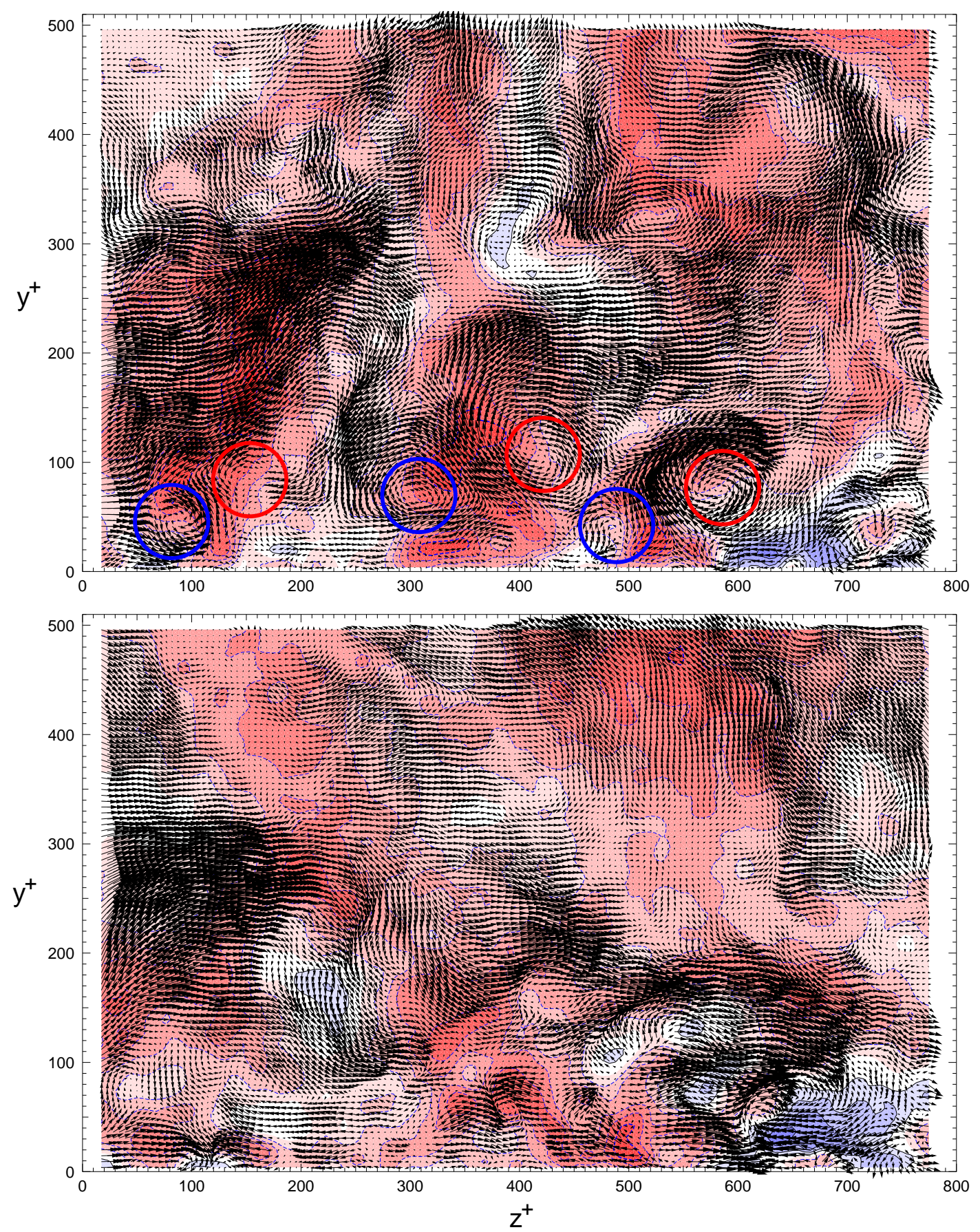

FIGURE 7.21: Same as figure 7.18 but measured simultaneously at spatially separated planes $\left(\Delta x^{+}=\right.$ 300 ) to examine the length of the stream-wise vortices in $x$-direction. 


\subsubsection{Stream-wise vortices}

In oder to examine the extent of the stream-wise vortices for different wall distances two pairs of velocity fields are shown in figure 7.21 and figure 7.22 which where measured simultaneously in spatially separated planes. The distance between both measurement planes is 325 wall-units. The first pair of velocity fields reveals hairpin like structures at a moderate wall distance, as indicated by the coloured circles in the top image. However, when the lower image is considered at the location where the vortices appear in the upper image, it is difficult to
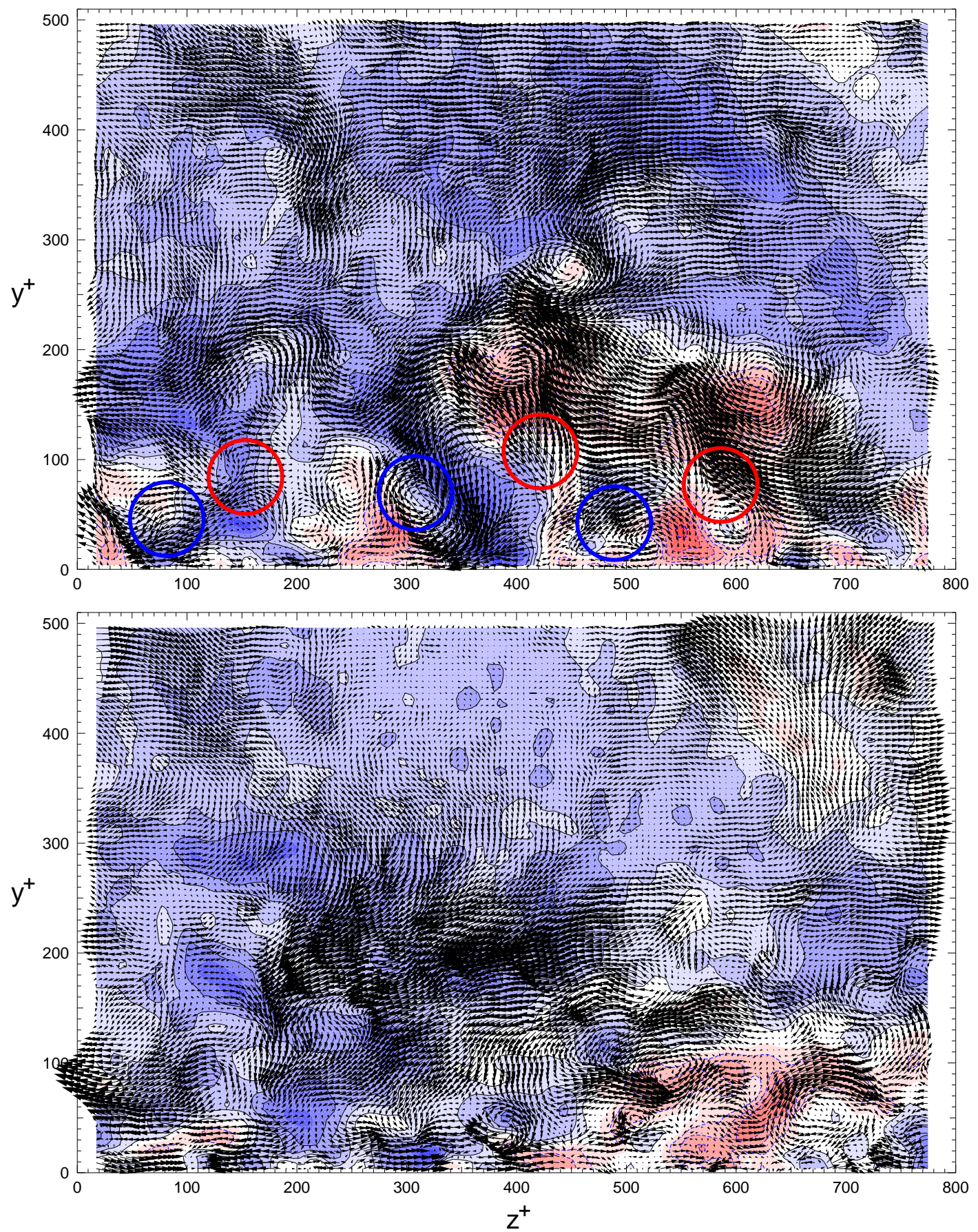

FIGURE 7.22: Same as figure 7.21. 
identify structural similarities. The same holds for figure 7.22 where especially the near-wall vortices below $y^{+}=100$ are considered. Thus, it can be again stated that these vortices are not usually several thousand wall-units in length as assumed by some authors. Another remarkable feature is the shear-layer indicated by the red contour in the lower right of figure 7.22 and the different height of this flow region between the image pair recorded simultaneously at different $x$ locations. Finally it can be seen that the low-momentum region is frequently flanked by stream-wise vortices. 
7 Investigation of the yz-plane 


\section{Summary}

The first part of this thesis examines different aspects of the Particle Image Velocimetry which are of fundamental importance for accurate measurements but which were not considered in detail in the literature. Starting point in chapter 2 is the general problem associated with the reproducible generation of sufficiently monodisperse particles with an appropriate size, shape and density that they follow the macroscopic flow motion faithfully without disturbing the flow or fluid properties. It could be shown quantitatively that high concentrations of particles with a narrow band size distribution and a mean diameter below $1 \mu \mathrm{m}$ can be generated best by means of multi-hole nozzles under over-critical pressure conditions ( $\geq 1$ bar), provided the kinetic energy, entering the liquid volume, is sufficient to change the fluid mechanical state of the liquid to a highly turbulent two-phase fluid. This experimental result was explained by assuming that the nozzle has basically three functions. First, it generates the particles at the nozzle exit. Secondly, it changes the fluid mechanical state of the fluid into a two phase liquid which seems to promote the generation of smaller particle size distributions, because the fluid mechanical parameters are different. Thirdly, the remaining kinetic energy which is not consumed for the generation of the particles or the transition of the fluid mechanical state is transferred into the turbulent motion of the liquid and it may act as an active impactor as the shearing of the bubbles which carry the particles to the liquid surface is enhanced. In order to examine the generation, transport and delivery of the particles inside the atomiser in detail, qualitative visualisation experiments were performed. It could be shown that bubbles arise from the liquid-feed holes of the Laskin nozzle. This implies that no particles can be generated at the ring side of the jet, in contrast to previous explanations in the literature.

Beside the fundamental problems associated with the generation of appropriate tracer particles, the registration, the storage and the read-out of the particle images were investigated in detail. This is another key element in PIV as the accuracy of the technique strongly depends on the precision with which the image displacement can be related to particle locations and their respective particle displacements. The discussion of the physical aspects of commonly used CCD sensors and electronics implies that the measurement noise in PIV is strongly affected by the geometrical properties of the picture elements but also by nonlinearities in the pixel response, the read-out of the images, gain and offset errors during the AD-conversion, amplification and transportation via long cable connections. It can be concluded from the analysis that the measurement error introduced by the CCD sensor increases with decreasing dimensions of the interrogation window. Thus, for accurate displacement estimation the size of the interrogation window should be selected in such a way that the errors introduced by the CCD are averaged out. In this case the remaining measurement error is mainly determined by the performance of the peak fitting routine for sub-pixel accuracy. This error was also investigated in the same chapter. It could be shown that under ideal experimental conditions this remaining error is approximately 0.1 pixel for all displacements when a two-dimensional Gaussian peak 
fit routine is applied. When a one-dimensional peak fit routine is applied, this error strongly depends on the exact sub-pixel displacement. This error was identified as the main cause for the so called peak-locking effect.

Chapter 3 focuses on different recording and evaluation methods for stereoscopic PIV. Of primary interest is the commonly used angular-displacement technique because the inherent drawback of this recording arrangement is the characteristic variation of the magnification factor across the field of view due to the oblique viewing direction. Beside a variation of the spatial resolution across the field of view along with a varying particle image density, serious problems usually arise when both cameras are located on one side of the light sheet. In this case, the size of each of a pair of measurement volumes considered for the calculation of the third velocity component is inversely proportional with respect to each other. This problem is usually solved by deforming the particle image field in such a way that the magnification becomes constant over the field of view. Unfortunately, this procedure requires some image interpolation scheme which increases the principal measurement error due to the CCD sensor and sub-pixel routine. In this thesis the performance of several interpolation schemes were compared in order to demonstrate the effect on the measurement noise. Based on this results it can be concluded that the additional measurement error introduced by the interpolation becomes comparable with other noise sources when a bilinear or more complex interpolation method is applied. However, in order to avoid the interpolation completely a novel evaluation scheme is proposed where the interrogation window is deformed instead of the measured image. In addition it is shown that another error is introduced when the interrogation windows from each of a pair of stereoscopic images do not correspond exactly to the same region of flow. Unfortunately, this error can be hardly avoided in any real experiment due to mechanical or thermal variation during the experiment, for example. As this measurement error is much larger with respect to any other error in PIV, a so called calibration validation method was developed which allows to compensate this error completely. This method can be applied to each acquired image pair to guarantee that everything involved in the measurement is unaffected by wind tunnel vibrations, thermal distortions e.g. for the duration of the measurement. This becomes important in noisy environments or for long acquisition times.

Chapter 4 examines the basic aspects of the multiplane stereo PIV technique which was developed to measure the temporal variation of the flow with high accuracy at any flow velocity. It could be demonstrated that this measurement technique is very reliable, robust and well suited for all kinds of applications, purely scientific as well as for industrially motivated investigations in large wind tunnels where acquisition time, optical access and observation distances are constrained. Furthermore, it is based on the conventional PIV equipment and the familiar evaluation procedure so that available PIV systems can easily be expanded. The advantage of this measurement system regarding to other imaging techniques lies in its ability to determine a variety of fundamentally important fluid-mechanical quantities with high accuracy (no perspective error) simply by changing the time sequence or the light sheet position. Another big advantage of this method becomes obvious, when the dynamic range of the particle image displacement is extremely large or when strong out-of-plane motions decrease the performance of standard and stereoscopic PIV. This is due to the fact that the performance and accuracy of the evaluation can be increased significantly when the information of the four independent particle image fields is used. Therefore various evaluation schemes are described in detail. A problem of this technique, which might occur, is caused by optical aberrations. Although it is impossible to completely eliminate all aberrations in any real system of finite 
aperture, it is shown how to eliminate certain aberrations by accepting aberrations of other types which are of no harm in PIV. This point is of primary importance because higher order aberrations like distortion and curvature of the field just influence the position and form of the image but do not lower the resolution. They can be completely eliminated numerically by using the methods examined in chapter 3 . Primary aberrations, on the other hand, like spherical aberration, coma and especially astigmatism deteriorate the image and alter the shape in a characteristic way. This leads to an increased measurement error because the performance of the peak-fit routine for sub-pixel accuracy strongly decreases for particle image diameter not equal 2-3 pixel. For completeness it should be mentioned at this point that the multiplane stereo PIV technique is frequently applied in other laboratories by now, see [77, 32] for example, and also commercially available.

The second part of this thesis reveals the main fluid mechanical results measured with the Multiplane Stereo PIV techniques in the temperature-stabilised, closed circuit wind tunnel at the Laboratoire de Mécanique de Lille (LML). In chapter 5 the stream-wise wall-normal plane ( $x y$-plane) of the turbulent boundary layer flow is examined at $R e_{\theta} \approx 7800$ and 15000 . To validate the flow characteristics and the performance and accuracy of the PIV technique, the basic statistical properties of the flow were determined at first and compared with the theoretical predictions in the literature and the hot-wire anemometry results discussed in $[11,21]$. The agreement with the analytical law of the wall and the log-law is excellent and also the hot-wire measurements match nicely with the PIV investigation when the mean velocity profile in outer- and inner-law scaling, the RMS-profiles of the three velocity fluctuations, the anisotropy parameter $\left(\overline{v^{2}} / \overline{u^{2}}\right)^{1 / 2}$ and the turbulence-level $\left(\overline{u^{2}}\right)^{1 / 2} / \bar{U}$ are considered, see page 76 to 78 . The shape, size and coherence of the turbulent flow structures and their dependence on the Reynolds number was estimated from the primary spatial correlations $R_{u u}, R_{v v}$ and $R_{w w}$ of the velocity fluctuations and $R_{\omega_{z} \omega_{z}}$, see page 80 to 87 . The similarity between the size of $R_{u u}$ and $R_{w w}$ in stream-wise direction and the elliptical shape around the maximum, accompanied with the general variation of the orientation with increasing wall distance of the fixed point, implies a strong relation between both fluctuations over several hundred wall units. However, the crestfallen shape of the $R_{w w}$ correlation indicates that the physical mechanism which connects the $u$ and $w$ fluctuations depends on the wall distance. This can be explained by the different shape of the coherent flow structures. While the near-wall region below $y^{+} \approx 50$ is dominated by well organised low-speed streaks, the log-law region above $y^{+} \approx 50$ is dominated by shear-layers and large-scale eddy structures. It is shown that the production of turbulence at $y^{+}>50$ is frequently associated with span-wise vortices which are located on top of the shear-layers. These vortices pump low-speed fluid from the shear-layer away from the wall. This process is associated with a relatively large velocity component in wall normal direction. However, the spatial extent of the region where $u v$ is negative is quite small. Large areas with $u v<0$ are frequently associated with relatively simple eddy structures (no vortices) which transfer high momentum fluid towards the wall as assumed in the mixing length theory by Prandtl, see page 91 to 94 . As the stream-wise momentum transported with this structure is quite large, their contribution to the production of turbulence is already significant when the negative wall-normal velocity component is relatively small. However, as the probability density function of the wall-normal velocity component is symmetrically within the log-law region, this process is compensated on average by a motion of high-speed fluid away from the wall. This changes in the near-wall region examined in chapter 6 . The analysis of the various double- and triple-correlations, which are associated with the production of tur- 
bulence, namely $R_{(u u)(u u)}, R_{(v v)(v v)}, R_{q q},-R_{u v},-R_{v u}, R_{(u v)(u v)}, R_{(u u v)(u u v)}, R_{(u v v)(u v v)}$ and $R_{(v w w)(v w w)}$ shows that the width of the correlations at a particular value decreases in general with increasing complexity of the correlations $R_{u u}>R_{(u u)(u u)}>R_{(u u v)(u u v)}$, see page 88 and 89. This implies a decreasing importance of the higher order correlations and establishes the simplifications usually applied in the formulation of conservation equations for the Reynolds stresses.

Chapter 6 illuminates the properties of the turbulent flow at $R e_{\theta} \approx 7800$ in stream-wise span-wise planes ( $x z$-planes) located at $y^{+} \approx 10,20,30$. This region is of primary interest according to chapter 5 because of the strong dynamic of the flow structures and the large production of turbulence. In order to obtain information about the structural features of the coherent structures, the size, shape and intensity of various spatial correlation, cross-correlation and conditional-correlation functions are examined in detail, see page 106 to 113. It is shown that the range of scales and span-wise periodicity of the coherent structures present in the flow depends strongly on the wall-distance. The mean streak-spacing is 92 wall-units at $y^{+} \approx 10$ when estimated from the conditional correlation, see page 108, and the span-wise size of the stream-wise vortices associated with sweeps measures 27 to 53 wall units at $y^{+} \approx 10,20,30$ while those associated with ejection are 35 to 42 wall units in size for the same wall locations, see page 107. However, the stream-wise size of these vortices is short relative to the length of the low-speed streaks, and it seems that these vortices are induced locally by the lift-up of low-speed streaks. This means that the stream-wise vortices which flank the low speed streaks are no primary vortices. They are produced when the streaks move away from the wall. The dynamics of the dominant structures is investigated by means of spatio-temporal correlation, cross-correlation and conditional correlations functions measured in spatially separated planes, see page 115 to 120 . The conditional correlations yield information about the space-time structure of the bursting phenomenon and allows to estimate the mean convection velocity of the coherent velocity structures present in the near wall region. The analysis of instantaneous velocity fields along with the probability density function of the Reynolds stress component $u v$ on page 121 implies, that most of the production of turbulence is associated with low-speed streaks, but the magnitude of the instantaneous Reynolds stress component $u v$ associated with streaks is relatively small, see page 123 and 124. The flow structures associated with large values of $|u v|$ on the other hand are frequently hair-pin like, see page 125 to 131. However, as the likelihood of these structures is quite small relative to the lifting streaks, they do not contribute to the total Reynolds stress to a large extend in the near wall region. The occurrence, intensity and main flow direction of the coherent structures is deduced from the analysis of the joint probability density function of the velocity fluctuations, see page 101 to 104. It is shown that the largest flow angles $\left(\alpha>10^{\circ}\right)$ in wall-normal direction are usually associated with ejection and sweeps, see page 105. In order to identify the structures responsible for the characteristic velocity pattern observed in hot-wire investigations, the velocity structure of the PIV measurements were analysed in stream-wise direction for various spanwise locations, see page 132 and 133. It was shown that the characteristic velocity pattern identified with the single point probes is caused by low-speed streaks. This could be further confirmed by comparing significant parameters with the results reported in the literature.

Chapter 7 reveals the results measured in the wall-normal span-wise plane (yz-plane) at $R e_{\theta} \approx 7800$ and 15000 . Of primary interest was the spatio-temporal dependence of the various correlation functions and the validity of Taylor's hypothesis because the interpretation of the results presented in chapter 6 was partially based on the assumption that the flow struc- 
tures keep their spatial organisation to a large extent while travelling down-stream by a few hundred wall units. The experimental results indicate that the maximum of the $R_{u u}$ correlation reach values above 0.8 for wall locations of the fixed point larger 100 wall units when the flow moves 300 wall units in stream-wise direction, see page 152 . For $y^{+} \approx 30$ the maximum reaches values above 0.6 . This implies that the structural features of the velocity pattern conserve their identity to a large extent. This justifies the assumption made for the interpretation of the results in chapter 6 . Furthermore, this result implies that the complex turbulent motion at a single point is a result of relatively simple coherent flow structures which are convecting downstream in form of a frozen pattern. In this sense, the complexity is a result of the spatial distribution of the structures and their orientation relative to the main flow direction and not a result of a strong structural changes of the flow field itself. To examine the interaction of the coherent flow structure below $y^{+}=100$ the cross-correlation $R_{v w}$ and $R_{w v}$ was calculated, see page 144 and 145 . These functions imply that a vertical motion towards the wall induces a horizontal motion away from the centreline due to continuity, and a vertical motion at the fixed point location with $v>0$ induces a horizontal motion towards $\Delta z^{+}=0$ in the near-wall region and away from the centre at higher wall locations. This correlated motion indicates that a motion away from the wall is associated with the generation of a stream-wise vortex pair in accordance with the interpretation in chapter 6 . To examine the characteristic features of the stream-wise vortices in detail and their significance for the turbulent mixing, instantaneous velocity fields were analysed, see page 156 to 160. It is shown that stream-wise vortices can be frequently observed in the near-wall region and also vortex pairs which transfer lowmomentum fluid away from the wall could be detected. However, the analysis shows that the stream-wise length of these vortices is not several thousand wall units in length, in agreement with the results in chapter 6 , and the number of vortices which could be detected was relatively small relative to the number of low-speed streaks present in the near-wall region. This implies that the streaks are not generally flanked by stream-wise vortices as assumed in the literature. 
8 Summary 


\section{Bibliography}

[1] ACARLAR MS, SMITH CR (1987) A study of hairpin vortices in a laminar boundary layer. Part 2. Hairpin vortices generated by fluid ejection, J. Fluid Mech. 175, pp. 43-83

[2] ADRIAN RJ (1988) Statistical properties of particle image velocimetry measurements in turbulent flow, in Laser Anemometry in Fluid Mechanics III. Springer-Verlag, Berlin Heidelberg, pp. 115-129

[3] ADRIAN RJ (1995) Limiting resolution of particle image velocimetry for turbulent flow, in Advances in Turbulence Research, Proc. 2nd Turbulence Research Assoc. Conf. Pohang Inst. Tech. pp. 1-19

[4] Adrian RJ, Meinhart CD, Tomkins CD (2000) Vortex organisation in the outer region of the turbulent boundary layer, J. Fluid Mech. 422, pp. 1-54

[5] BARnhart DH, AdRIAN RJ, PAPEN GC (1994) Phase-conjugate holographic system for high-resolution particle image velocimetry, Appl. Optics 33, pp. 7159-7170

[6] Bergmann L, SchäFER C (2004) Optik, Lehrbuch der Experimentalphysik Bd. 3, de Gruyter, Berlin New York

[7] BlackWELder RF, ECKelmann H (1979) Streamwise vortices associated with the bursting phenomenon, J. Fluid Mech. 132, pp. 87-103

[8] Born M, Wolf E (1985) Principles of Optics, Press, 6th edition

[9] BROWN GR, THOMAS ASW (1977) Large structures in a turbulent boundary layer, Phys. Fluids 20, pp. 243-251

[10] BRÜCKER C (1997) 3D scanning PIV applied to an air flow in a motored engine using digital high-speed video, Meas. Sci. Tech. 8, pp. 1480-1492

[11] Bruns J, Dengel P, Fernholz HH (1992) Mean flow and turbulence measurements in an incompressible two-dimensional turbulent bounday layer. Part I: Data, Institutsbericht Nr. 02/92, Herman-Föttinger-Institut für Thermo- und Fluiddynamik, Technische Universität Berlin.

[12] Carlier J, Foucaut JM, Dupont P, Stanislas M (1999) Caractérisation d'une couche limite turbulente à grand nombre de Reynolds par anémométrie à fils chauds et par Vélocimétrie par Images de Particules, 35ème colloque d'Aérodynamique Appliquée. Analyse et contrôle des écoulements turbulents, Lille, France 
Bibliography

[13] CARLIER J, KÄHLER CJ, STANiSlas M (2001) Experimental study of eddy structures in a turbulent boundary layer using PIV, 4th Intl. Symp. on Particle Image Velocimety, Göttingen, Germany, Sept. 17-19

[14] Di Cicca GM, Iuso G, SPazzini PG, Onorato M (2002) PIV study of the influence of large-scale stream-wise vortices on a turbulent boundary layer, Exp. Fluids 33, pp. 663-669

[15] CORINO ER, BRODKEY RS (1969) A visual investigation of the wall region in turbulent flow, J. Fluid Mech. 37, pp. 1-36

[16] Dreeben TD, Pope SB (1997) Probability density function and Reynolds-stress modeling of near wall turbulent flows Phys. Fluids 9, pp. 154-163

[17] Echols WH, Young YA (1963) Studies of portable air-operated aerosol generators, NRL Report 5929, Naval Research Laboratory, Washington D.C.

[18] Ehrenfried K. (2002) Processing calibration-grid images using Hough transformation, Meas. Sci. Tech. 13, pp. 975-983

[19] FaVre AJ, GaViglio JJ, Dumas RJ (1957) Space-time double correlations and spectra in a turbulent boundary layer, J. Fluid Mech. 2, pp. 313-342

[20] Favre AJ, Gaviglio JJ, Dumas RJ (1958) Further space-time correlations of velocity in a turbulent boundary layer, J. Fluid Mech. 3, pp. 344-356

[21] FeRnhOlz HH, FInley PJ (1996) The incompressible zero-pressure-gradient turbulent boundary layer: An assessment of the data, Prog. Aerospace Sci. 32, pp. 245-311

[22] Fincham A, Delerce G (1999) Advanced optimization of correlation imaging velocimetry algorithms, 3rd International Workshop on PIV, University of California, Santa Barbara, USA, Sept. 16-18

[23] Gebelein H (1935) Turbulenz, Springer-Verlag, Berlin

[24] GRAnt HL (1971) The large eddies of turbulent motion, J. Fluid Mech. 4, pp. 149-190

[25] GUPTA AK, LAUfER J, KAPLAN RE (1971) Spatial structure in the viscous sublayer, J. Fluid Mech. 50, pp. 493-512

[26] HAMA FR (1962) Streaklines in a perturbed shear flow, Phys. Fluids 5, pp. 644-650

[27] HART DP (2000) PIV error correction, in Laser Techniques Applied to Fluid Mechanics, ed. RJ Adrian et al , Springer-Verlag, Berlin Heidelberg, pp. 19-36

[28] HEAD MR, BANDYOPADHYAY P (1981) New aspects of turbulent boundary-layer structure, J. Fluid Mech. 107, pp. 297-338

[29] Hinsch K (1993) Particle image velocimetry (PIV), in Speckle Metrology, ed. RS Sirohi, Marcel Dekker, New York, pp. 235-324 
[30] Hinsch K (1995) Three-dimensional particle velocimetry, Meas. Sci. Tech. 6, pp. 742753

[31] HinZE JO (1975) Turbulence, 2nd ed., McGraw-Hill New York

[32] Hu H, Sata T, Kobayashi T, Taniguchi N, Yasuki M (2001) Dual-plane stereoscopic particle image velocimetry: system set-up and its application on a lobed jet mixing flow, Exp. Fluids 31, pp. 277-293

[33] Huang HT, Fiedler HE, WANG JJ (1993) Limitation and improvement of PIV, Exp. Fluids 15, pp. 168-174 and pp. 263-273

[34] ISO 13320-1 (1999) Particle size analysis - Laser diffraction methods, First edition, 1999-11-01, Reference number: ISO 13320-1:1999(E)

[35] JÄHnE (1997) Digital image analysis, 4th edition, Springer-Verlag, Berlin Heidelberg

[36] Jakobsen ML, Dewhirst TP, Greated CA (1997) Particle image velocimetry for predictions of acceleration force within fluid flows, Meas. Sci. Tech. 8, pp. 1502-1516

[37] JISCHA M (1982) Konvektiver Impuls-, Wärme- und Stoffaustausch, Vieweg \& Sohn. Braunschweig Wiesbaden

[38] KäHLER CJ (1997) Ortsaufgelöste Geschwindigkeitsmessungen in einer turbulenten Grenzschicht, Diplomarbeit, Deutsche Forschungsanstalt für Luft- und Raumfahrt, Research Report DLR-FB 97-32.

[39] KäHLER CJ (2000) Multiplane stereo PIV - Recording and evaluation methods, Proc. EUROMECH 411, Rouen, France, May 29-31

[40] KäHLER CJ (2001) Stereoscopic PIV, German Aerospace Center, Lecture Series, Application of Particle Image Velocimetry - Theory and Practice, Göttingen, Germany

[41] KÄHLER CJ (2004) Investigation of the spatio-temporal flow structure in the buffer region of a turbulent boundary layer by means of multiplane stereo PIV, Exp. Fluids 36, pp. 114-130

[42] KäHLER CJ (2003) Experimental investigation of flows at low Reynolds number using optical techniques, von Karman Institute for Fluid Dynamics, RTO/AVT-104 VKI Lecture Series on Low Reynolds number aerodynamics on aircraft including applications in emerging UAV technology, Rhode-Saint Genèse, Belgium, Nov. 24-28

[43] KäHLER CJ (2003) General design and operating rules for seeding atomisers, 5th International Symposium on Particle Image Velocimety, Busan, Korea, September 2224, paper 3207

[44] KÄHLER CJ, AdRIAN RJ, WILlERT CE (1998) Turbulent boundary layer investigations with conventional and stereoscopic PIV, Proc. 9th Intl. Symp. on Appl. of Laser Techniques to Fluid Mechanics, Lisbon Portugal, paper 11.1, June 13-16 
[45] Kähler CJ, Kompenhans J (1999) Multiple plane stereo PIV - Technical realization and fluid-mechanical significance, 3rd International Workshop on PIV, University of California, Santa Barbara, USA, paper PIV96, Sept. 16-18

[46] Kähler CJ, Kompenhans J (2000) Fundamentals of Multiple Plane Stereo PIV, Exp. Fluids [Suppl.], pp. S70-S77

[47] Kähler CJ, SAmmler B, Kompenhans J (2002) Generation and Control of Particle size distributions for Optical Velocity Measurement Techniques in Fluid Mechanics, Exp. Fluids 33, pp. 736-742

[48] KÄHLER CJ, SCHOlz U (2003) Investigation of laser-induced flow structures with time-resolved PIV, BOS and IR technology, 5th International Symposium on Particle Image Velocimety, Busan, Korea, September 22-24, paper 3223

[49] Kähler CJ, Stanislas M, Dewhirst TP, Carlier J (2001) Investigation of the spatio-temporal flow structure in the log-law region of a turbulent boundary layer by means of multi-plane stereo particle image velocimetry, in Laser Techniques to Fluid Mechanics, ed. RJ Adrian et al , Springer-Verlag, Berlin Heidelberg, pp. 39-53

[50] KASPERSEN(1996) A study of coherent structures using wavelet analysis. Thesis, University of Science and Technology, Trondheim, Norway

[51] KeAne RD, AdRIAn RJ (1990) Optimization of particle image velocimeters. Part I: Double pulsed systems, Meas. Sci. Tech. 1, pp. 1202-1215

[52] KEANE RD, ADRIAN RJ (1992) Theory of cross-correlation analysis of PIV images, Appl. Sci. Res. 49, pp. 191-215

[53] Khintchine A (1933) Korrelationstheorie der stationären stochastischen Prozesse, Math. Annalen. 109, pp. 604-615

[54] Kim HT, Kline SJ, ReYNOLDS WC (1971) The production of turbulence near a smooth wall in a turbulent boundary layer, J. Fluid Mech. 50, pp. 133-160

[55] KlebanOfF PS (1955) Characteristics of turbulence in a boundary layer with zero pressure gradient, NACA TR-1247, pp. 1135-1153

[56] KLEIN F (1925) Elementarmathematik vom höheren Standpunkte aus, Bd. 2, 3rd edition, Springer-Verlag, Berlin

[57] KLINE SJ (1978) The role of visualization in the study of the structure of the turbulent boundary layer, Coherent Structures of the Turbulent Boundary Layers. Ed Smith C.R. \& Abbot D.E., AFOSR/Lehigh, pp. 1-26

[58] Kline SJ, Reynolds WC, Schraub FA, Runstadler PW (1967) The structure of turbulent boundary layers, J. Fluid Mech. 30, pp. 741-773

[59] KLIne SJ, RUnSTADLER PW (1959) Some preliminary results of visual studies of the flow model of the wall layers of the turbulent boundary layer, Trans. ASME. Ser. E. Vol. 2 
[60] KNEubÜHL FK, SigRIst MW (1995) Laser, Teubner Verlag, Stuttgart

[61] KREPLIN H-P, ECKELMANN H (1978) Instantaneous direction of the velocity vector in a fully developed turbulent channel flow, Phys. Fluids 22 (6), pp. 1210-1211

[62] Kreplin H-P, Eckelmann H (1979) Propagation of perturbations in the viscous sub-layer and adjacent wall region, J. Fluid Mech. 95, pp. 305-322

[63] Labraga L, LagraA B, Mazouz A, Keirsbulck L (2002) Propagation of shearlayer structures in the near-wall region of a turbulent boundary layer, Exp. Fluids 33, pp. 670-676

[64] Landau LD, Lifschitz EM (1978) Statistische Physik, Lehrbuch der Theoretischen Physik Bd. 5, Akademie-Verlag, Berlin

[65] Landau LD, LifSChITz EM (1978) Physikalische Kinetik, Lehrbuch der Theoretischen Physik Bd. 10, Akademie-Verlag, Berlin

[66] Landhal MT, Mollo-Christensen E (1986) Turbulence and random processes in fluid mechanics, Cambridge University Press

[67] LASKIn S (1948) Submerged Aerosol Unit, AEC Project Quarterly Report UR-38, Univ. of Rochester.

[68] LAUFER J (1953) Investigation of turbulent flow in a two-dimensional channel, NACA TR-1053, pp. 1247-1266

[69] LAUFER J (1954) The structure of turbulence in fully developed pipe flow, NACA TR$1174,417-434$

[70] Lauterborn W, Kurz T, Wiesenfeldt M (1995) Coherent Optics, SpringerVerlag, Berlin Heidelberg

[71] LAWSON NJ, WU J (1997) Three dimensional particle image velocimetry: error analysis of stereoscopic techniques, Meas. Sci. Tech. 8, pp. 894-900

[72] LECORDIER B, LECORDIER JC, TRINITE M (1999) Iterative sub-pixel algorithm for the cross-correlation PIV measurements, 3rd International Workshop on PIV, University of California, Santa Barbara, USA, Sept. 16-18

[73] LESIEUR M (1996) Turbulence in Fluids, Kluwer-Verlag

[74] MeInHART CD (1994) Investigation of turbulent boundary-layer structure using particle-image velocimetry, Ph.D. dissertation, Department of Theoretical and Applied Mechanics. Urbana Illinois

[75] Melling A (1997) Tracer particles and seeding for particle image velocimetry, Meas. Sci. Tech. 8, pp. 261-304

[76] Monin AS, Yaglom AM (1973) Statistical fluid mechanics, The MIT Press, Cambridge, Mass. 
Bibliography

[77] Mullin JA, DAHM W (2002) Highly-resolved three dimensional velocity measurements via Dual-Plane Stereo Particle Image Velocimetry (DSPIV) in turbulent flows, AIAA Paper No. 2002-0290

[78] Noack BR, Dallmann U (1995) On Models and Theories for Turbulent Flows, Deutsche Forschungsanstalt für Luft- und Raumfahrt, Research Report DLR-IB 22395 A33

[79] Nychas SG, Hershey HC, Brodkey RS (1973) A visual study of turbulent sher flow, J. Fluid Mech. 61, pp. 513-540

[80] Oesterlund JM (1999) Experimental studies of zero pressure-gradient turbulent boundary layer flow, PhD thesis, Department of Mechanics, Royal Institute of Technology, Stockholm, Sweden

[81] OfFEn GR, KLINE SJ (1974) Combined dye-streak and hydrogen-bubble visual observations of a turbulent boundary layer, J. Fluid Mech. 104, pp. 223

[82] PERRY AE (1984) A study of degenerate and nondegenerate critical points if threedimensional flow fields, Deutsche Forschungs- und Versuchsanstalt für Luft- und Raumfahrt, Research Report DFVLR-FB 84-36.

[83] Perry AE, Lim TT, TeH EW (1980) A visual study of turbulent spots, J. Fluid Mech. 62, pp. 387-405

[84] PRATURI AK, BRODKEY RS (1978) A stereoscopic visual study of coherent structures in turbulent shear flow, J. Fluid Mech. 89, pp. 251-272

[85] Raffel M, Willert CE, Kompenhans J (1998) Particle Image Velocimetry - A practical Guide, Springer-Verlag, Berlin Heidelberg

[86] RoBINSON SK (1989) A review of vortex structures and associated coherent motions in turbulent boundary layers, 2. IUTAM Symposium on Structures of Turbulence an Drag Reduction. Federal Institute of Technology. Switzerland Juli 25-28

[87] RoBINSON SK (1991) The kinematics of turbulent boundary layer structure, NASA Technical Memorandum Number 103859

[88] Ronneberger O, RAFFel M, Kompenhans J (1998) Advanced evaluation algorithms for standard and dual plane particle image velocimetry, 9th Int. Symp. on Appl. of Laser Techn. to Fluid Mechanic Lisbon Portugal, paper 10.1, June 13-16

[89] RotTA JC (1962) Turbulent Boundary Layers in Incompressible Flow, Reprint from "Progress in Aeronautical Science, Volume 2". Pergamon Press Oxford

[90] RotTA JC (1972) Turbulente Strömungen, B.G. Teubner-Verlag Stuttgart

[91] Schlichting H (1951) Grenzschicht-Theorie, Verlag G. Braun. Karlsruhe. 1. Auflage

[92] Schraub FA, Kline SJ (1965) Mech. Eng. Dept. Rept. MD-12, Stanford University 
[93] SMith CR, METZLER SP (1983) The characteristics of low-speed streaks in the nearwall region of a turbulent boundary layer, J. Fluid Mech. 120, pp. 27-54

[94] SMith CR, WALKeR DA (1999) Turbulent wall-layer vortices, in Fluid Vortices, Green SI (ed.), Kluver, 235-290

[95] SOlOFF SM, AdRIAN RJ, ZIU Z-C (1997) Distortion compensation for generalized stereoscopic particle image velocimetry, Meas. Sci. Tech. 8, pp. 1441-1454

[96] SPALART PR (1988) Direct simulation of a turbulent boundary layer up to $R_{\theta}=1410$, J. Fluid Mech. 187, pp. 61-98

[97] Stanislas M, OKamoto K, Kähler CJ (2003) Main results of the First International PIV Challenge, Meas. Sci. Tech. 14, pp. R1-R27

[98] TheOdorsen T (1955) The Structure of Turbulence, In 50 Jahre Grenzschichtforschung, Görtler/Tollmien (ed.)

[99] Townsend AA (1976) The Structure of Turbulent Shear Flow, Cambridge Monographs on Mechanics and Applied Mathematics 2nd. Edition

[100] TRITTON DJ (1967) Some new correlation measurements in a turbulent boundary layer, J. Fluid Mech. 28, pp. 439-462

[101] Virant M, Dracos T (1997) 3D PTV and its application on Lagrangian motion, Meas. Sci. Tech. 8, pp. 1539-1552

[102] Wallace JM, Brodkey RS, Eckelmann H (1977) Pattern-recognized structures in bounded turbulent shear flow, J. Fluid Mech. 83, pp. 673-692

[103] Wallace JM, EcKelmann H, Brodkey RS (1972) The wall region in turbulent shear flow, J. Fluid Mech. 54, pp. 39-48

[104] WeSterWeEL J (1997) Fundamentals of digital particle image velocimetry, Meas. Sci. Tech. 8, pp. 1379-1392

[105] WESTERWEEL J (1998) Effect of sensor geometry on the performance of PIV interrogation, in Laser Techniques Applied to Fluid Mechanics, ed. RJ Adrian et al, SpringerVerlag, Berlin Heidelberg, pp. 37-55

[106] Westerweel J, DABIRI D, GHarib M (1997) The effect of a discrete window offset on the accuracy of cross-correlation analysis of digital PIV recordings, Exp. Fluids 23, pp. 20-28

[107] WiEgEL M (1996) Experimentelle Untersuchung von kontrolliert angeregten dreidimensionalen Wellen in einer Blasius-Grenzschicht, Dissertation Universität Hannover

[108] WILLERT CE (1997) Stereoscopic digital particle image velocimetry for application in wind tunnel flows, Meas. Sci. Tech. 8, pp. 1465-1479

[109] Willert CE, GHARIB M (1991) Digital particle image velocimetry, Exp. Fluids 10, pp. 181-193 
Bibliography

[110] Willmarth WW (1975) Structure of turbulent boundary layers, Adv. Appl. Mech. 15, pp. 159-254

[111] Willmarth WW, LU SS (1975) Structure of the Reynolds stress near the wall, J. Fluid Mech. 55, pp. 65-92

[112] ZHOU J, ADRIAN RJ, BALACHANDAR S (1996) Auto-generation of near-wall vortical structures in channel flow, Phys. Fluids 8, pp. 288-290 


\section{Commonly used symbols}

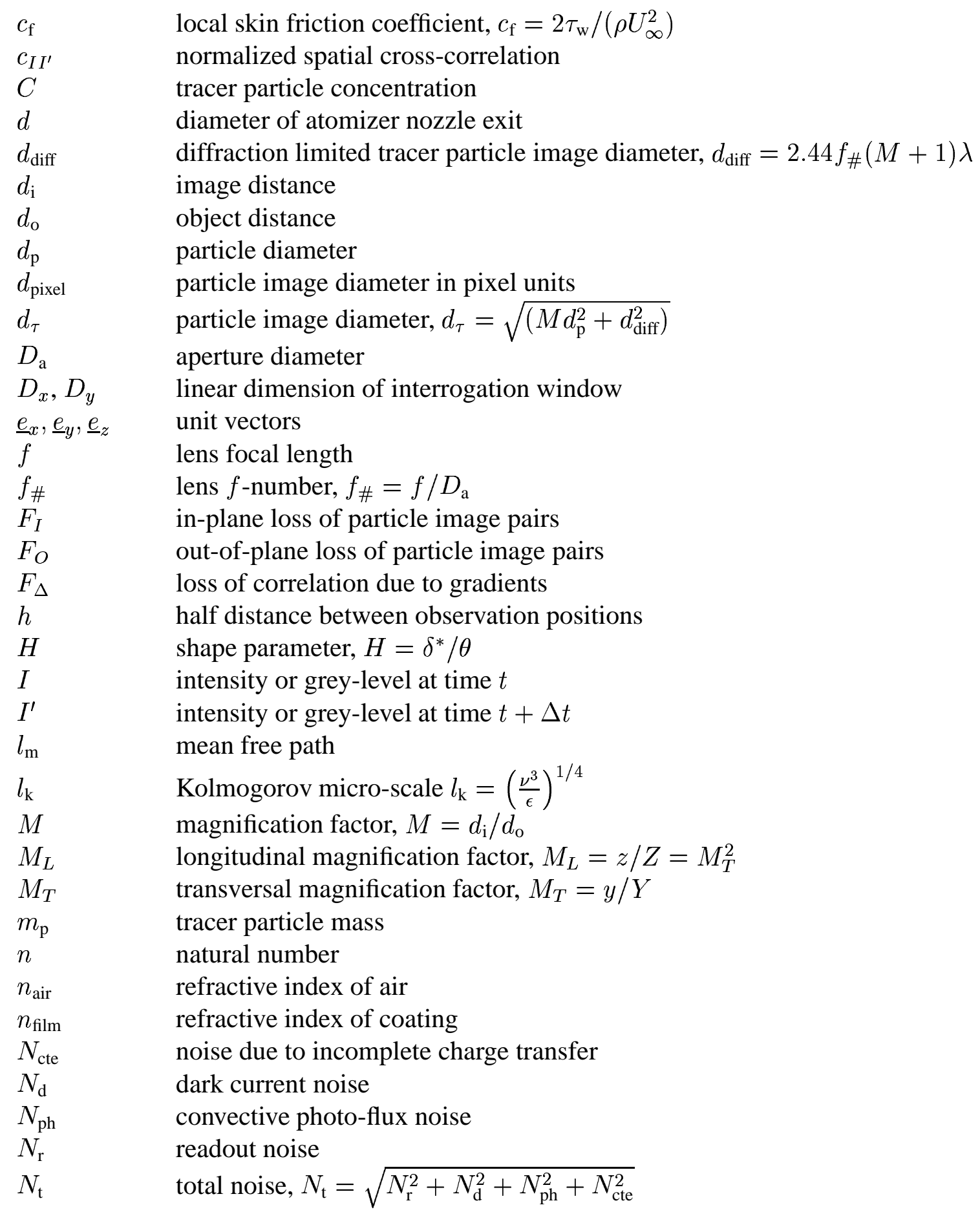




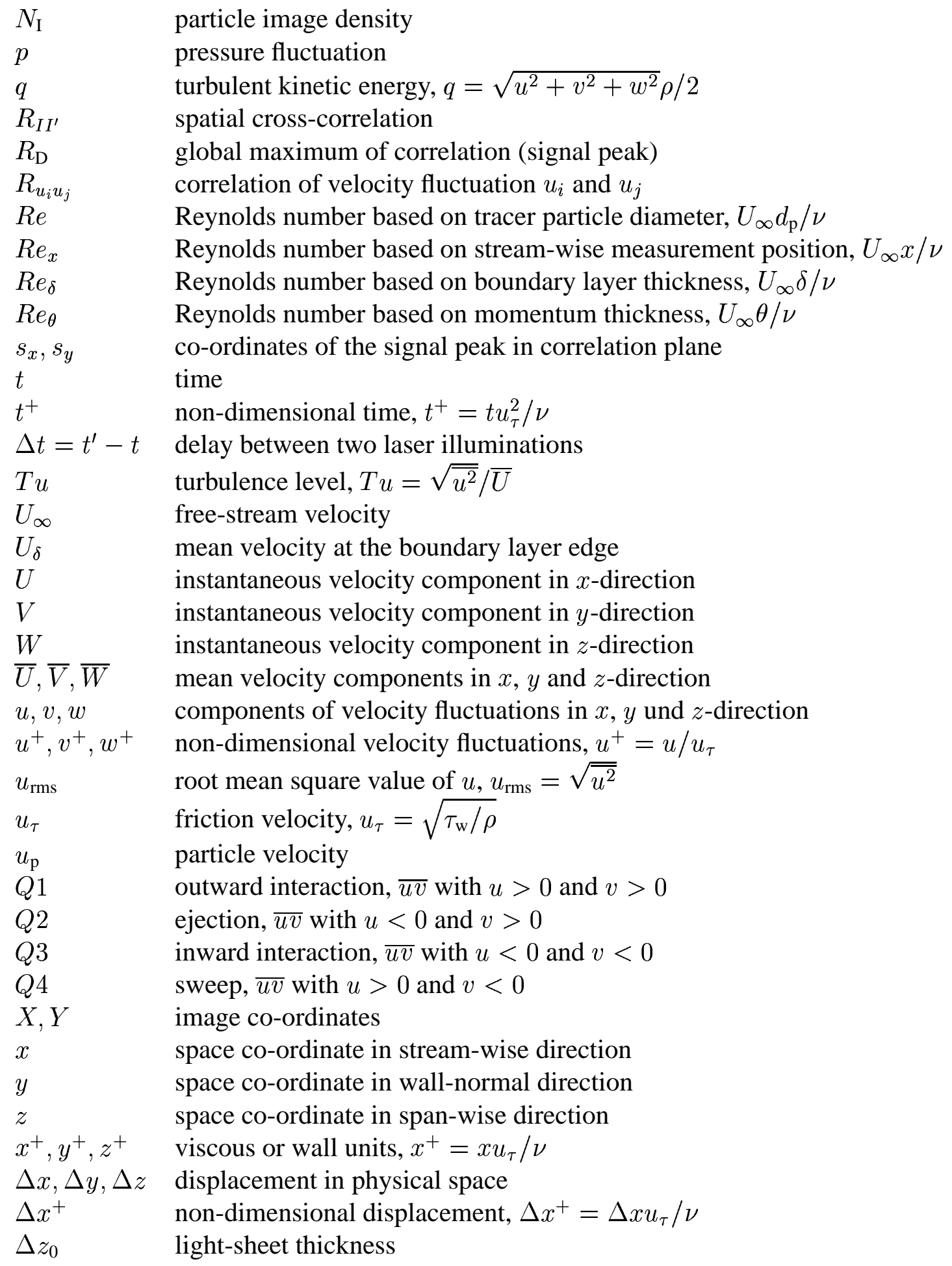

\section{Greek symbols}

$\begin{array}{ll}\alpha & \text { off-axis angle } \\ \delta & \text { boundary layer thickness } \delta=\delta\left(\bar{U}=0.99 U_{\infty}\right) \\ \delta_{z} & \text { depth of focus, } \delta_{z}=2 f_{\#} d_{\text {diff }}(M+1) / M^{2}\end{array}$




$\begin{array}{ll}\delta^{+} & \text {non-dimensional boundary layer thickness } \\ \delta^{*} & \text { displacement thickness, } \delta^{*}=\int_{0}^{\infty}\left(1-\bar{U} / U_{\infty}\right) d y \\ \epsilon & \text { total energy } \\ \theta & \text { momentum thickness, } \theta=\int_{0}^{\infty} \bar{U} / U_{\infty}\left(1-\bar{U} / U_{\infty}\right) d y \\ \kappa & \text { VON KÁRMÁN constant } \\ \Lambda_{i j} & \text { integral correlation length, } \Lambda_{i j}=\int_{0}^{\infty} R_{i j} d\left(\Delta x_{i}\right) \\ \lambda & \text { wavelength of laser light } \\ \lambda_{i j} & \text { dissipation length, } \lambda_{i j}=1 / \sqrt{-\frac{1}{2}} \frac{\partial^{2} R_{i j}}{\partial \Delta x^{2}} \\ \lambda^{+} & \text {non-dimensional streak spacing in } z \text {-direction } \\ \mu & \text { dynamical viscosity } \\ \nu & \text { kinematical viscosity, } \nu=\mu / \rho \\ \rho & \text { density of the fluid, } 1.2 \mathrm{~kg} / \mathrm{m}^{3}(\text { air }) \\ \rho_{\mathrm{p}} & \text { tracer particle density, } 874 \mathrm{~kg} / \mathrm{m}^{3}\left(\text { olive oil), } 912 \mathrm{~kg} / \mathrm{m}^{3}(\mathrm{DEHS})\right. \\ \sigma_{a} & \text { standard deviation of index }(\text { here } a) \\ \tau_{\text {mol }} & \text { molecular shear stress, } \tau_{\text {mol }}=\mu \partial \bar{U} / \partial y \\ \tau_{\text {tur }} & \text { turbulent shear stress, } \tau_{\text {tur }}=-\rho \overline{u v} \\ \tau_{\mathrm{w}} & \text { wall shear stress, } \tau_{\mathrm{w}}=\lim y \rightarrow 0 \partial \bar{U} / \partial y \\ \omega_{z} & \text { components of the vorticity fluctuation in } z \text {-direction }\end{array}$

\section{Abbreviations}

CCD Charge Coupled Device

DLR Deutsches Zentrum für Luft- und Raumfahrt e.V.

DNS Direct Numerical Simulation

DPIV Digital Particle Image Velocimetry

FFT Fast Fourier Transformation

HFI Hermann Föttinger Institut

HWA Hot-Wire Anemometer

LML Laboratoire de Mecanique de Lille

LES Large Eddy Simulation

MPSPIV Multiplane Stereo Particle Image Velocimetry

PDF Probability Density Function

PIV Particle Image Velocimetry

RANS Reynolds Averaged Navier-Stokes Simulation

RMS Root Mean Square

SPIV Stereo-scopic Particle Image Velocimetry 


\section{Index}

A

aberration ...................6 61

accuracy .................. 15, 16

aerosol atomiser ................ 16

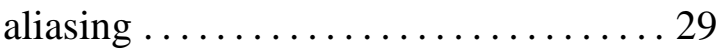

angular displacement method.........38

anisotropy parameter............. 78

astigmatism ..................6 61

B

beam-splitter . . . . . . . . . . . . . . . 50

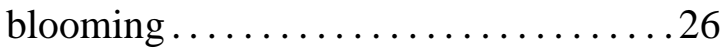

boundary conditions ..............6 69

buffer-layer.................... 71

bursting phenomena............. 122

C

calibration.......................99

charge coupled device (CCD) . . . . . . . 24

charge transfer...................25

circular effects ................... 29

closure problem................. 72

coating ....................... 59

convection..................... 72

correlation

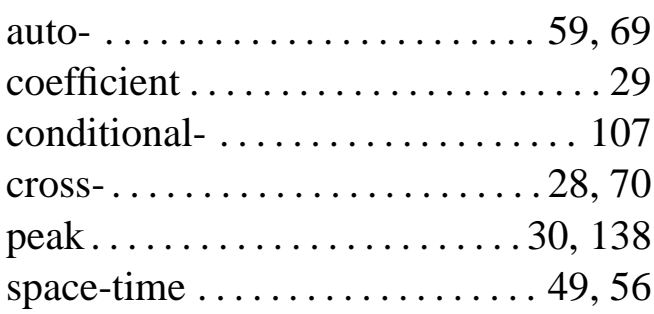

D

dark current noise ................. . 25

depth of focus .................. 41

diffraction limited image ........ 26, 61

digital recording $\ldots \ldots \ldots \ldots \ldots \ldots . \ldots 24$

discretisation .................... 24

distortion ................. 42, 61
$\mathbf{E}$

eddies ................ 5, 69, 95

ejection..................93, 122

ergodic theorem ............. 30, 70

evaluation ....................... 28

\section{F}

fast Fourier transformation . . . . . . . . . . 29

feed holes ..................... 16

fill-ratio .................... 25-27

flow

disturbance ............. 5, 65

visualisation ............. 5, 21

fluid element ............... 13, 69

fluid volume ................... 28

forward scattering $\ldots \ldots \ldots \ldots \ldots 15,38$

friction velocity ............... 76

frozen pattern hypothesis .......... 133

fully developed flow ..............78

\section{G}

ghost-image ..............661, 66

grey-level interpolation ............ . 44

grey-level quantisation...............24

\section{I}

image density ................... 30

image interpolation methods ......... 44

impactor ...................... 19

inrush bursts................... 122

instabilities ..................... 69

integration time ................ 25

intermittency ................. 5, 71

interrogation

area.....................28

window .................... 29

J

joint probability density...........70

K

Kelvin-Helmholtz instability .....91, 122 
Kolmogorov micro-scale

69

$\mathbf{L}$

laminar boundary layer $\ldots \ldots \ldots \ldots .5,65$

laser.....................15, 51

Laskin nozzle .............. 16, 21

law-of-the-wall . . . . . . . . . . . . . . . 71

light-sheet .................... 14

log-law ..................... 71

loop-shaped structure $\ldots \ldots \ldots \ldots 6,155$

loss-of-pairs . . . . . . . . . . . . . . 30

low-speed streak............6, 121

\section{M}

magnification $14,38,42$

mean value

mean velocity profile..........76, 138

Mie scattering ................. 15

molecular stress .................71

momentum thickness ............... 75

multi-pass interrogation..............31

\section{O}

opening angle...................39

out-of-plane

component ................. 37

error...................39, 46

outer-flow effects ............... 76

$\mathbf{P}$

particle

concentration .............14, 16

density .................. 15

displacement.................15

image..................26, 61

image displacement ........... 15

size ....................... 16

tracer-....................13

pattern recognition technique........ 129

peak-looking .................. 58

perspective error ................. 37

photographic recording ............ 24

picture element (pixel)..............24

pixel storage capacity..............26

point spread function .............. 26

polarisation....................60 60

probability density $\ldots \ldots \ldots \ldots \ldots \ldots 69$
Q

quantum efficiency ..............25

\section{$\mathbf{R}$}

read-out......................25

reflection .............. 15, 26, 59

retardation plate................51

Reynolds

equation ................... 70

number...................... 5

stress equation $\ldots \ldots \ldots \ldots \ldots \ldots 72$

stress tensor ................. 70

\section{$\mathbf{S}$}

sampling theorem ............... 24

Scheimpflug condition ......... 42, 50

sequencer ................... 54

shear-layer .................6, 60

signal-to-noise ratio ...........26, 31

smoke generator ............... 17

spatial resolution ................ 15

spherical aberration ...............6 61

statistical ensemble................699

storage-capacity ................ 26

streak-spacing ................. 108

stream-wise vortices . . . . . . . . . . . 6

sub-pixel accuracy ............... 31

suction nozzle ................. 16

sweep .................. 95, 122

\section{$\mathbf{T}$}

Taylor hypothesis............ 114, 135

transition ....................... 7

translation method ............... 38

tripping device $\ldots \ldots \ldots \ldots \ldots \ldots 7,75$

turbulence-level ............. 7, 78

turbulent

boundary layer .............. 5

diffusion ................... 72

dissipation ............. 5, 72

kinetic energy............... 72

length scales ................. 79

mixing $\ldots \ldots \ldots \ldots \ldots \ldots \ldots \ldots \ldots \ldots \ldots \ldots$

production ............. 72, 122

redistribution ............... 72

spectrum ............... 49 


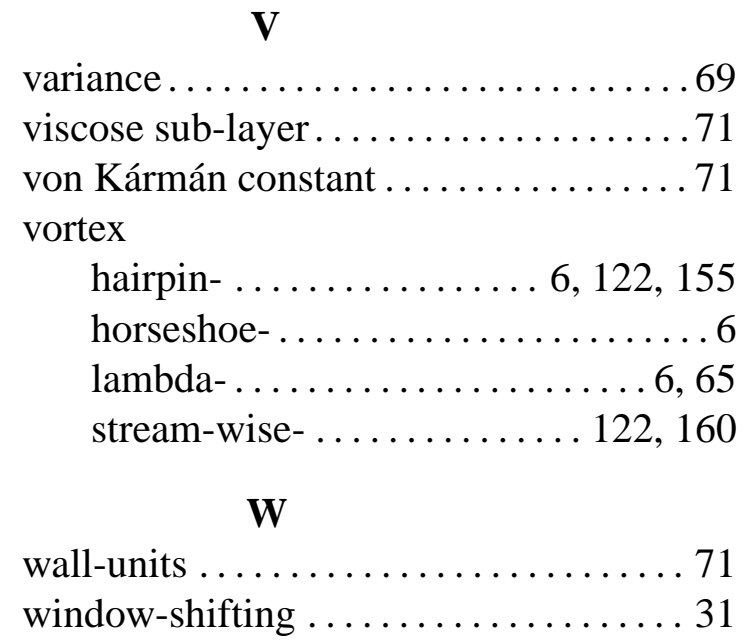




\section{Acknowledgement}

First of all I would like to thank Prof. Dr. Eckelmann from the University of Göttingen, who has always been ready to discuss experimental and fluid mechanical questions and to give invaluable advice, and thanks to Prof. Dr. Ronneberger for the comments on the draft version of this book.

Secondly, I would like to thank Dr. Bütefisch and Dr. Kompenhans from the German Aerospace Center (DLR) for their professional and financial support in any situation, without that this work would have never been finished and I would also express my gratitude to both for their continuing interest in my research work over the years and the strong scientific promotion that I have experienced while I was working at DLR.

Thirdly, I would like to thank Prof. Dr. Stanislas from the Laboratoire de Mécanique de Lille (LML) in France, especially for his experimental support, without that the second part of this book could neither have been conceived nor carried out, and I would like to thank Prof. Dr. Fernholz from the Hermann Föttinger Institut (HFI) in Berlin for the provision of the hot-wire results.

In addition I would like to express my thanks to all colleagues at DLR and around the world who have directly or indirectly influenced the success of this thesis, especially Dr. Kreplin and Dr. Dewhirst.

Finally, I would like to address my sincere gratitude to my wife Ariane, my son Florian and my mother for their never-ending patience, understanding and love and I wish I could also thank my father to whom I dedicate this work in honour. 


\section{Lebenslauf}

von Christian Joachim Kähler, geboren am 5.1.1969 in Buchholz i.d. Nordheide als zweites Kind von Joachim Kähler und Christina Kähler, geb. Behrens. Staatsangehörigkeit: Deutsch

Juli 1989 Allgemeine Hochschulreife.

April 1990 Registrierung an der Technischen Universität in Clausthal im Studiengang Physik. Schwerpunkt: Atom-, Molekül-, Festkörperphysik und Strömungsmechanik.

August 1995 bis April 1997 Diplomarbeit an der Technischen Universität Clausthal. Thema: Ortsaufgelöste Geschwindigkeitsmessungen in einer turbulenten Grenzschicht. Durchgeführt im Institut für Strömungsmechanik der Deutschen Forschungsanstalt für Luft- und Raumfahrt (DLR) in Göttingen.

April 1997 Doktorand an der Georg August Universität zu Göttingen. Angestellter am Institut für Strömungsmechanik des Deutschen Zentrums für Luft- und Raumfahrt in Göttingen. Forschungsgebiete: Untersuchung turbulenter Grenzschichten und Weiterentwicklung der Particle Image Velocimetry.

Januar 2000 Wissenschaftlicher Angestellter am Institut für Strömungsmechanik des Deutschen Zentrums für Luft- und Raumfahrt in Göttingen. Forschungsgebiete: Weiterentwicklung der Mehrebenen- und Holographischen Particle Image Velocimetry für Untersuchungen in grossen Windkanälen.

Oktober 2001 Wissenschaftlicher Angestellter am Institut für Strömungsmechanik der Technischen Universität Braunschweig. Leiter der Abteilung Strömungsbeeinflussung und Messtechnik.

University of Illinois at Urbana Champaign (UIUC), Dept. of Theoretical and Applied Mechanics, 216 Talbot Laboratory, 101 South Wright Street, Urbana, Illinois 61801-2935, USA, March 18 - June 181996.

California Institute of Technology (Caltech), Graduate Aeronautical Laboratories 205-45, Pasadena, California 91125, USA, September 6 - December 171998.

Ecole Centrale de Lille, URA CNRS 1441, Cité Scientifique, BP 48, 59651 Villeneuve d'Ascq, Frankreich, May 21 - June 61999. 